\title{
WHOI-93-17
}

\section{Hydrodynamics and Morphodynamics of Shallow Tidal Channels and Intertidal Flats}

\author{
by \\ Carl T. Friedrichs \\ Woods Hole Oceanographic Institution \\ Woods Hole, Massachusetts 02543 \\ and \\ The Massachusetts Institute of Technology \\ Cambridge, Massachusetts 02139 \\ February 1993

\section{DOCTORAL DISSERTATION} \\ Education and the National Science Foundation under grant OCE91-02429. \\ MIT/WHOI, WHOI-93-17.
}

Funding was provided by the Office of Naval Research through the American Society for Engineering

Reproduction in whole or in part is permitted for any purpose of the United States Government. This thesis should be cited as: Carl T. Friedrichs, 1993. Hydrodynamics and Morphodynamics of Shallow Tidal Channels and Intertidal Flats. Ph.D. Thesis.

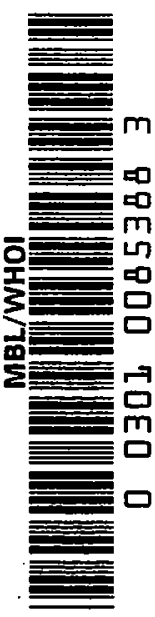

Approved for publication; distribution unlimited.

Approved for Distribution:

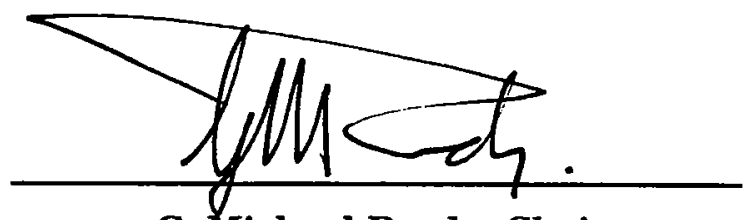

G. Michael Purdy, Chair

Department of Geology and Geophysics

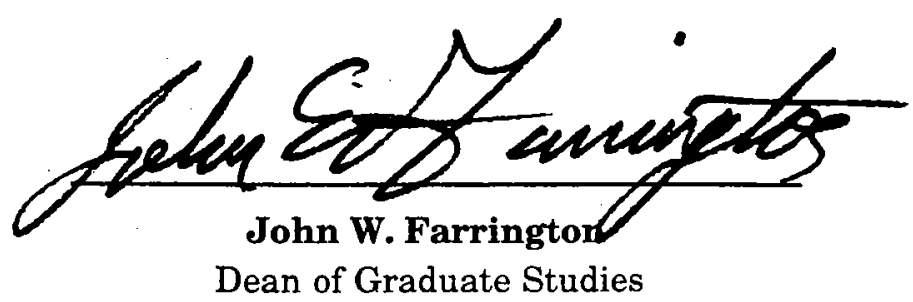


HYDRODYNAMICS AND MORPHODYNAMICS OF SHALLOW TIDAL CHANNELS AND INTERTIDAL FLATS

by

CARL TAKEO FRIEDRICHS

B.A., Amherst College (1986)

Submitted in partial fulfillment of the requirements for the degree of

DOCTOR OF PHILOSOPHY

at the

MASSACHUSETTS INSTITUTE OF TECHNOLOGY

and the

WOODS HOLE OCEANOGRAPHIC INSTITUTION

February, 1993

(C) Carl T. Friedrichs, 1993

The author hereby grants to MIT and WHOI permission to reproduce and distribute copies of this thesis document in whole or part.

Signature of Author $\quad$ Cas T Fiedinch

Joint Program in Oceanography Massachusetts Institute of Technology /

Certified by avid Senior Scientist, Wobds Hole Oceanographic Institution Thesis Supervisor

Accepted by

Marcia K. MaOut

Marcia K. McNutt

Professor, Massachusetts Institute of Technology Chair, Joint Committee for Marine Geology and Geophysics 


\title{
HYDRODYNAMICS AND MORPHODYNAMICS OF SHALLOW TIDAL CHANNELS AND INTERTIDAL FLATS
}

by

\section{Carl Takeo Friedrichs}

Submitted to the Massachusetts Institute of Technology-Woods Hole Oceanographic Institution Joint Program in Oceanography, February 1993, in partial fulfillment of the requirements for the degree of Doctor of Philosophy

\begin{abstract}
The ultimate goal of this study is to relate the hydrodynamics of shallow tidal embayments to patterns of natural evolution and morphologic equilibrium. The specific problems addressed in this thesis are largely motivated by two mechanisms previously identified as major controls on net sediment transport in shallow tidal systems: temporal and spatial asymmetries in maximum bottom shear stress $(\tau)$. In the process of investigating these mechanisms, important aspects of basic mass and momentum balances in these systems are also revealed.

A new perturbation scheme is applied to nonlinear propagation in shallow embayments which identifies and quantifies the mechanisms ultimately responsible for temporal asymmetries in $\tau$. This new scheme, which employs a perturbation in time (but not space) is simpler than previous methods, is consistent with available observations, and maintains the fundamental features of "exact" numerical solutions. This approach allows the major geometric properties controlling tidal asymmetry to be combined into a single non-dimensional parameter, $\gamma$. Solutions for overtides which determine asymmetry are compact and easily interpreted. For $\gamma>0$, time-varying depth plays a larger role in determining asymmetry than time-varying width, and the rising tide is of shorter duration. For $\gamma<0$, time varying width plays a larger role, and the falling tide is of shorter duration.

Morphologic implications of the spatial distributions of $\tau$ are also investigated. Observations of cross-sectional area along many tidal channels are observed to be consistent with equilibrium models based on a uniform distribution of $\tau$. The critical stress just capable of initiating sediment motion is found to provide a lower bound on $\tau$, and the characteristic value of $\tau$ appropriate to individual systems is found to be a function of spring tidal range. Small along-channel deviations away from uniform $\tau$ are associated with along-channel variation in the direction of maximum discharge. Uniform $\tau$ is then
\end{abstract}


used to derive equilibrium hypsometries (the distribution of basin area as a function of elevation) for intertidal flats. Domination of $\tau$ by either tidal currents or wind waves is found to favor convex or concave hypsometries, respectively, a trend which is consistent with empirical observations. In addition to uniform $\tau$, the effect of shoreline curvature on equilibrium hypsometry is also found to be important.

The investigation of mechanisms which control embayment evolution also reveals important aspects of basic hydrodynamic balances in shallow tidal embayments. A scaling relevant to prismatic channels having strong tidal asymmetries indicates friction often dominates acceleration in the momentum equation. The resulting balance between pressure gradient and friction gives a single time-varying diffusion equation for tidal elevation which only permits tidal amplitude to decay along channel. This result, which is consistent with observations and numerical solutions, diverges from classical cooscillation. Classical co-oscillation with (weak) friction suggests amplitude should oscillate through nodes and anti-nodes due to the interaction of incident and reflected waves.

Uniform $\tau$ is used to justify a new scaling of the continuity equation for exponentially-shaped channels. In tidal channels having a nearly uniform distribution of $\tau$ (such as the Delaware, Thames and Tamar), along-channel gradients in velocity are small and discharge gradients in the continuity equation are dominated by gradients in crosssectional area. With this scaling, the resulting governing equation is a first-order wave equation. The solution is a constant amplitude, forward propagating waveform which is independent of channel length -- in contrast to the length sensitive resonance of classical co-oscillation. Amplitude can grow or decay if higher order effects are taken into account, but these effects are due to variations in the rate of channel convergence rather than interactions between incident and reflected waves.

Thesis Supervisor: $\quad$ Dr. David G. Aubrey

Title: Senior Scientist

Woods Hole Oceanographic Institution 


\section{Acknowledgements}

Above all, I would like to thank my advisor Dave Aubrey for his guidance and unwavering support, extending back almost eight years to when I first came to Woods Hole as a Summer Student Fellow. His enthusiasm for oceanography was contagious and is largely responsible for my present career choice.

I am also grateful for the support and advice given me by the other members of my thesis committee: John Boon, Dan Lynch and Ole Madsen. Many of the ideas expressed in this thesis were inspired by conversations with and previous work by John, Dan and Ole. Knowledge gained in Ole's excellent courses at the Parsons Lab was also invaluable. I also thank John Trowbridge who acted as my defense chairman and provided helpful comments on this thesis.

I am grateful to Susan Berteaux and Colleen Hurter of the WHOI library staff for their assistance. Much of the historical data presented in this thesis was dug out of the "gray" literature with their help. Thanks also go to Pam Barrows, Chris Bradley, Gail Christeson, Gary Jaroslow, Tom McSherry, Elise Ralph, Wayne Spencer, Chris Weidman, and the staff of the WHOI Education Office, all of whom were gladly willing to drop what they were doing and help me out with any problems from data analysis or software bugs to writer's block or administrative red tape.

Finally I thank my wife, Marjorie, for her love and constant encouragement.

My work as a graduate student was funded by the Office of Naval Research through the American Society for Engineering Education, by the Woods Hole Oceanographic Institution, and by the National Science Foundation under grant OCE 9102429. Some information contained in the chapters of this thesis has appeared elsewhere. Chapter 2 was published with O. Madsen in the Journal of Geophysical Research, vol. 97, pp. 5637-5650 (reprinted here with permission from the American Geophysical

Union). Chapter 3 was presented at the 6th International Conference on the Physics of Estuaries and Coastal Seas, with D. Aubrey and D. Lynch as co-authors. Chapter 5 was presented at the same conference with D. Aubrey as co-author. 


\section{Table of Contents}

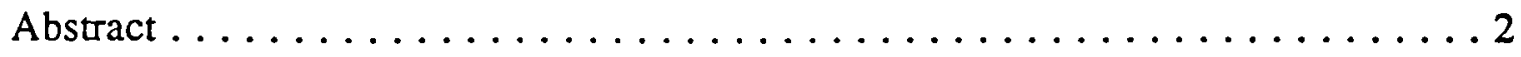

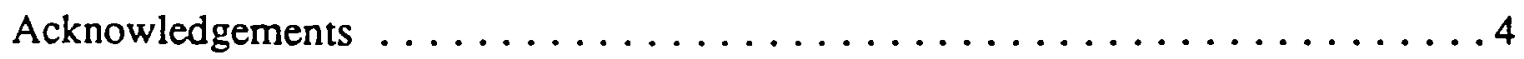

Chapter 1. Introduction $\ldots \ldots \ldots \ldots \ldots \ldots \ldots \ldots \ldots \ldots \ldots$

1.1. Hydrodynamic controls on temporal asymmetries in $\tau \ldots \ldots \ldots \ldots 10$

1.2. Morphologic ramifications of spatial asymmetries $\tau \ldots \ldots \ldots \ldots 11$

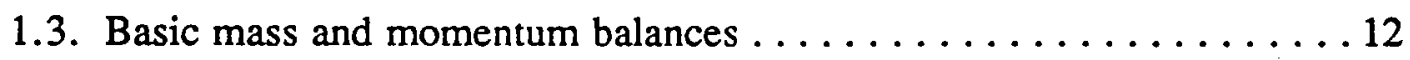

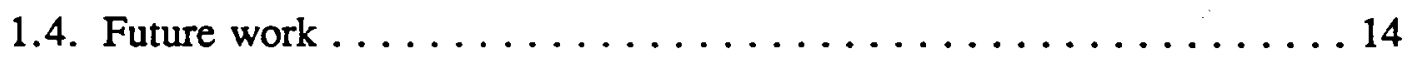

Chapter 2. Non-linear diffusion of the tidal signal in frictionally dominated

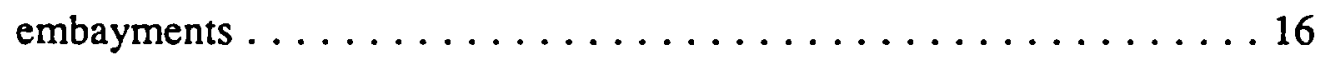

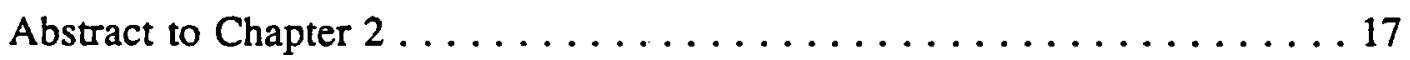

List of symbols in Chapter $2 \ldots \ldots \ldots \ldots \ldots \ldots \ldots \ldots \ldots \ldots \ldots \ldots \ldots \ldots \ldots$

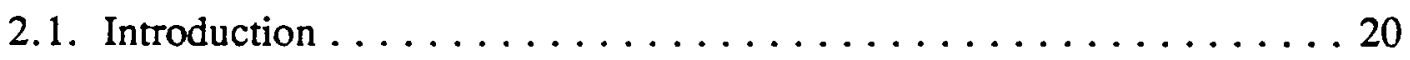

2.1.1. The frictional dominance assumption $\ldots \ldots \ldots \ldots \ldots \ldots 21$

2.1.2. Derivation of the zero-inertia equation. ............23

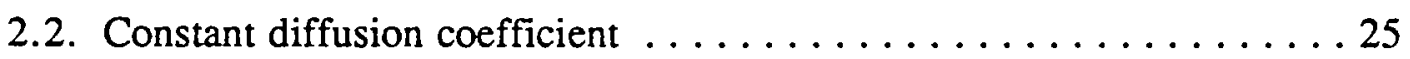

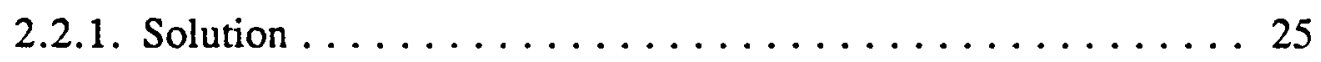

2.2.2. Nature of the constant coefficient solution .......... 26

2.3. Time-varying diffusion coefficient $\ldots \ldots \ldots \ldots \ldots \ldots \ldots \ldots 28$

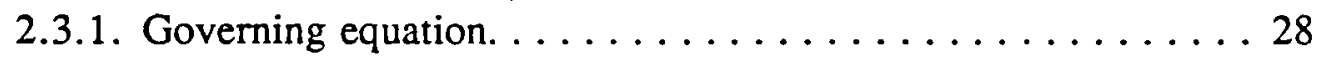

2.3.2. Solution . . . . . . . . . . . . . . . . . . . . 29

2.3.3. Nature of the time-varying coefficient solution ........ 31

2.4. Comparison to numerical solutions and observations .......... 33

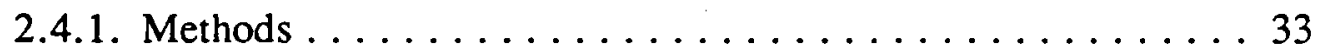

2.4.2. Numerical solutions . . . . . . . . . . . . 34

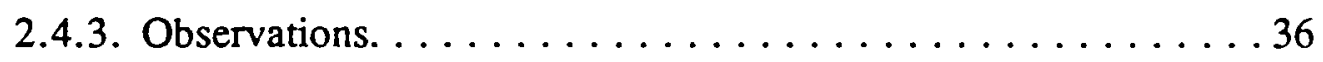

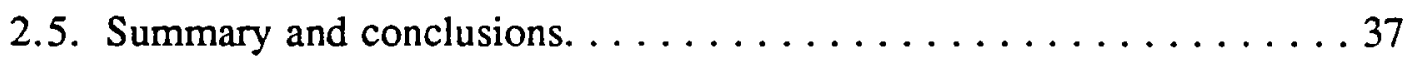

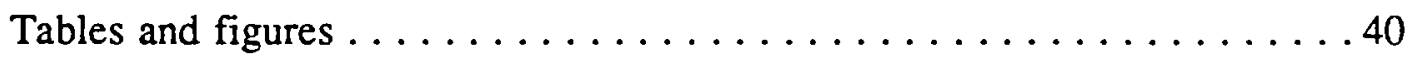

Chapter 3. Stability shear stress and equilibrium geometry of tidal channels and

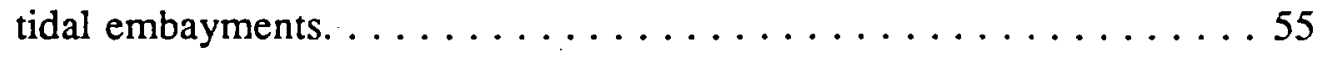

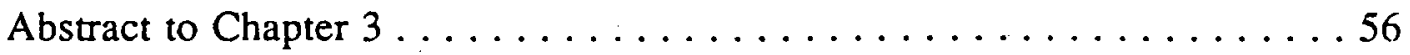

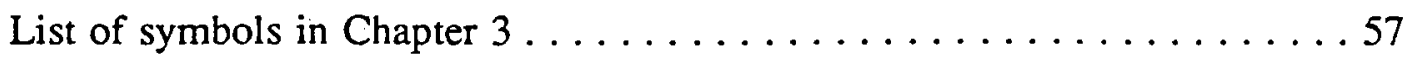

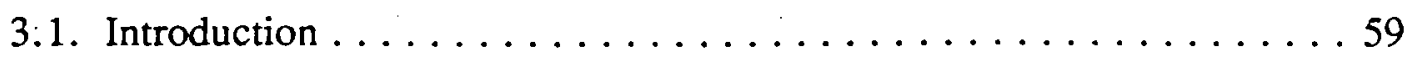


3.1.1. Previous observations of equilibrium tidal channels . . . . . . 60

3.1.2. Previous explanations for proportionality of area and discharge . . 62

3.2. Stability shear stress given by critical shear stress . . . . . . . . 63

3.2.1. Insight from unidirectional flow. . . . . . . . . . 63

3.2.2. Application to tidal channels . . . . . . . . . . . 64

3.2.3. Comparison to observations . . . . . . . . . . 66

3.3. Deviations of stability shear stress from critical shear stress. . . . . . . 69

3.3.1. Deviations among channels . . . . . . . . . . . . 69

3.3.2. Along-channel deviations . . . . . . . . . . . 71

3.4. Application of stability shear stress to entire embayments . . . . . . 73

3.4.1. Theory. . . . . . . . . . . . . . . . . . . 73

3.4.2. Comparison to observations . . . . . . . . . . 75

3.5. Further implications of uniform stability shear stress . . . . . . . . 76

3.6. Summary and conclusions. . . . . . . . . . . . . . 78

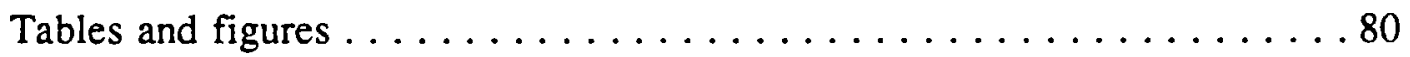

Chapter 4. Tidal propagation in strongly convergent channels near morphologic equilibrium. . . . . . . . . . . . . . . . . 98

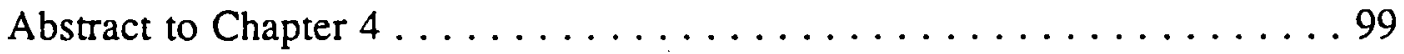

List of symbols in Chapter $4 \ldots \ldots \ldots$. . . . . . . . . . . 100

4.1. Introduction . . . . . . . . . . . . . . . . . . . . . 102

4.1.1. Classical tidal co-oscillation . . . . . . . . . . . . 102

4.1.2. Previous solutions for convergent tidal channels with friction . . 104

4.2. Scaling arguments . . . . . . . . . . . . . . . . 106

4.2.1. Scaling of continuity . . . . . . . . . . . 107

4.2.2. Scaling of momentum. . . . . . . . . . . 110

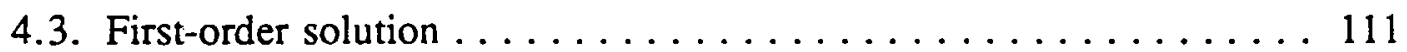

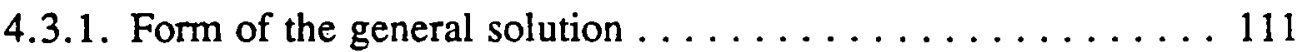

4.3.2. Exponential channel convergence . . . . . . . . . . 113

4.3.3. Discussion of first-order solution . . . . . . . . . . 114

4.4. Second-order solution. . . . . . . . . . . . . . . . . . 117

4.4.1. Derivation of the governing equation. . . . . . . . 117

4.4.2. General solution . . . . . . . . . . . . . . . . 119

4.5. Discussion of the second-order solution for tidal elevation. . . . . . . 122

4.5.1. Dominant tidal frequency. . . . . . . . . . . . . 122

4.5.2. Tidal harmonics. . . . . . . . . . . . . . . . . . . 125 


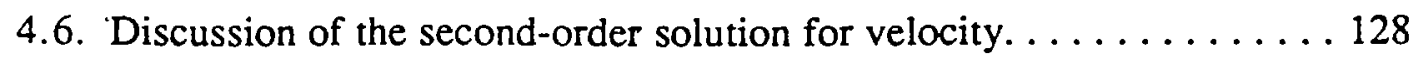

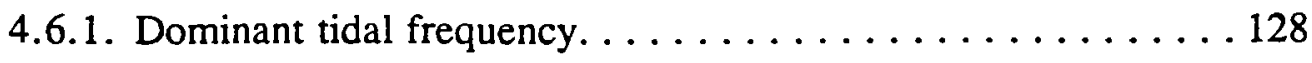

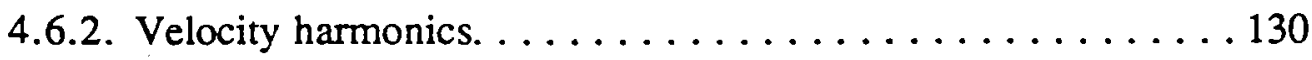

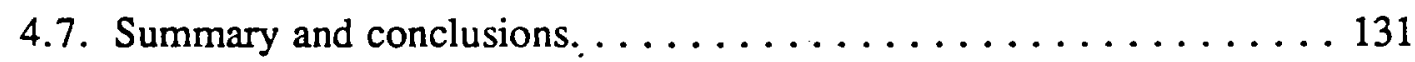

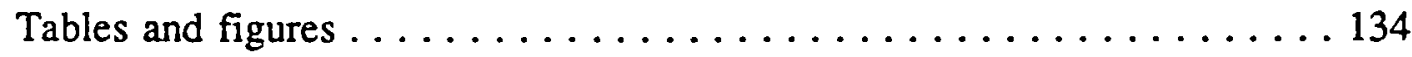

Chapter 5. Equilibrium hypsometry of intertidal flats. . . . . . . . . 150

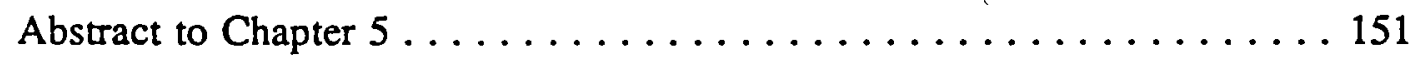

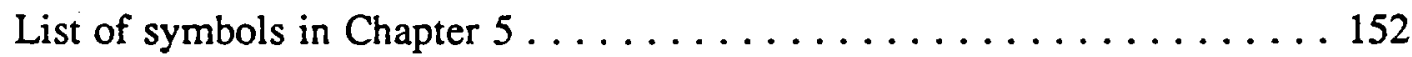

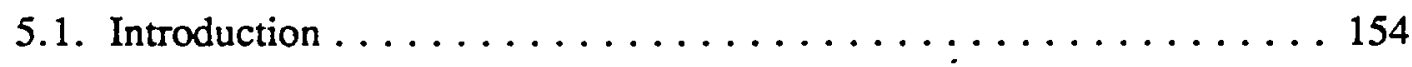

5.1.1. Definitions and previous work. ............ 154

5.1.2. Morphodynamic model. . ............... 156

5.1.3. Scaling of problem: southwest coast of Korea. . . . . . 157

5.2. Tidal currents . . . . . . . . . . . . . . . . 159

5.2.1. Straight shoreline, linear profile. ............ 160

5.2.2. Straight shoreline, equilibrium profile $\ldots \ldots \ldots \ldots \ldots \ldots 162$

5.2.3. Curved shoreline, equilibrium profile . . . . . . . . 164

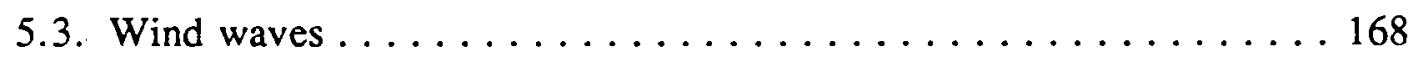

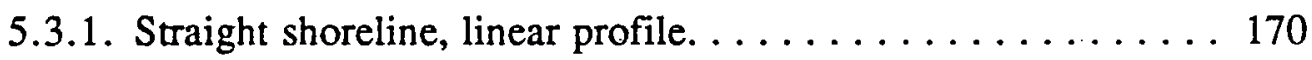

5.3.2. Straight shoreline, equilibrium profile $\ldots \ldots \ldots \ldots \ldots \ldots 171$

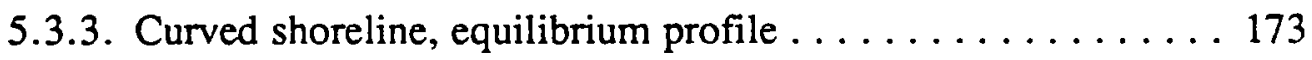

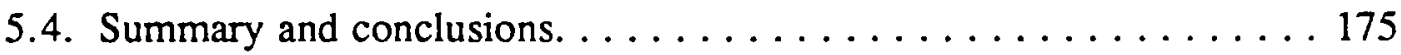

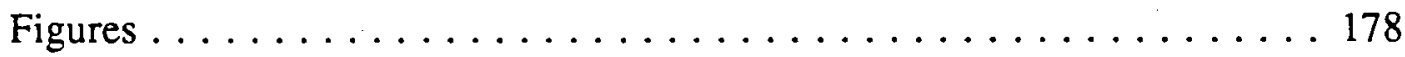

Appendices . . . . . . . . . . . . . . . . . . . . . . . . . . 194

A1. Solution of the zero-inertia diffusion equation for embayments with variation in along-channel geometry. . . . . . . . . . . . 195

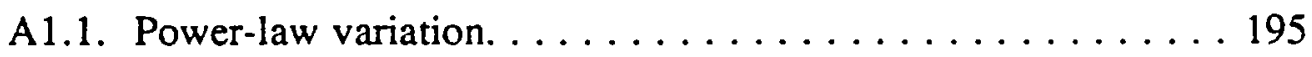

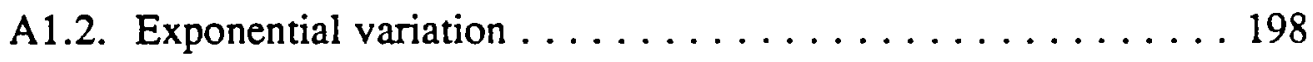

A2. Transformation of time domain in second-order zero-inertia equation. . . 200

A2.1. Transformation from $t$ to $\tau \ldots \ldots \ldots \ldots \ldots \ldots \ldots 200$

A2.2. Transformation from $\tau$ to $t \ldots \ldots \ldots \ldots \ldots \ldots \ldots \ldots \ldots$

A3. Derivation of the second harmonic relative phase in strongly convergent channels near morphologic equilibrium. . . . . . . . 202

A3.1. $\eta_{2}$ to $\eta_{1}$ relative phase. .................. 202

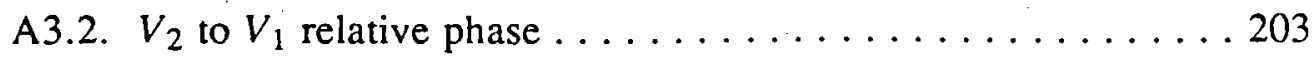

References. . . . . . . . . . . . . . . . . . . . . 204 
$\|(10-8-$

Chapter 1:

Introduction

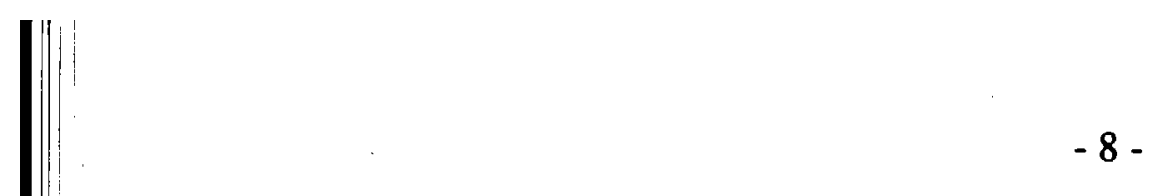


More than half of the world's non-arctic coastlines are either macrotidal (range > $4 \mathrm{~m}$ ) or mesotidal (range 2-4 m) (Davies 1980). Tides can be expected to play a significant role in determining morphology along these coastlines, especially where the coast is embayed by estuaries or fringed by barrier islands (Hayes 1979). Tides can also play a major role in determining morphology in microtidal regions (range $<2 \mathrm{~m}$ ) that are sheltered from wave activity, such as the Chesapeake Bay and its tributaries (Wright et al. 1987). The range of scales of interest to this thesis is broad, from the short, channelized embayments which are especially common along the east coast of the United States, to large tidal channels, such as the Delaware or Thames, having lengths of a hundred kilometers or more. Within these environments tidal channels and flats may occur together, as along the Ord River in Western Australia (Wright et al. 1973) and in the back barrier lagoon at Wachapreague Inlet, Virginia (Boon and Byrne 1981). Large tidal channels, such as the Delaware Estuary (Parker 1984), are also found without extensive intertidal flats, and large flats can occur without extensive channel networks, such as those bordering the southwestern coast of Korea (Alexander et al. 1991).

This thesis examines the hydrodynamics and morphodynamics of these tidal environments with the ultimate goal of relating physically-based mechanisms to patterns of natural evolution and morphologic equilibrium. Of particular interest are feedback processes where embayment geometry determines flow patterns, and energetic flows simultaneously constrain geometry. Descriptive reviews of shallow tidal systems (e.g., Hayes 1979; Klein 1985; Nichols and Biggs 1985) have identified morphologies which are correlated to specific forcings, but these studies are limited by their qualitative approach. At the same time, deterministic modeling from first principles (e.g., Yalin 1977; Mehta 1984) is hampered in natural tidal environments by broad spectra of length and time scales and by fundamental uncertainties concerning long-term mechanisms of net sediment transport. This thesis attempts to tread a useful middle ground via the application of simplified, yet quantitative, analytic models.

The specific problems addressed in this study are largely motivated by two mechanisms previously identified as major controls on net sediment transport in shallow tidal systems: temporal and spatial asymmetries in maximum bottom shear stress $(\tau)$. Controls on temporal asymmetries in $\tau$ are addressed through a simplified, yet quantitative, hydrodynamic investigation. Implications of spatial asymmetries in $\tau$ are investigated through stability criteria based on a uniform spatial distribution of $\tau$ at 
equilibrium. In the process of investigating asymmetries in $\tau$, important aspects of basic mass and momentum balances in these systems are also revealed.

Analytic approaches to tidal problems can be of limited value if results are not compared to available observations. Without the constraint of observations, analytic formulations may be poorly scaled or may emphasize processes which are not important in real tidal systems. In preparing this thesis, the literature was reviewed for relevant observations (see Tables in Chapters 2 to 4, for example). The result is not only reassurance that theoretical predictions bear resemblance to actual conditions, but also a compilation of valuable morphologic and tidal information for a range of geometrically varied embayments.

\subsection{Hydrodynamic controls on temporal asymmetries in $\tau$}

The hydrodynamic investigations in this thesis are largely inspired by continued interest in geometric controls on temporal tidal asymmetries. During the last two decades numerous studies have related temporal asymmetries in tidally-induced bottom shear stress to patterns of net sediment transport along channelized tidal embayments (e.g., Wright et al. 1975; Allen et al. 1980; Boon and Byrne 1981; Aubrey 1986; Dronkers 1986). Recently, temporal tidal asymmetries have been related successfully to specific embayment geometries through the use of diagnostic one-dimensional numerical models. By modeling specific embayment geometries, Speer and Aubrey (1985) and Friedrichs and Aubrey (1988) showed flood dominance to be associated with large fluctuations in channel depth during the tidal cycle and ebb dominance to be associated with large fluctuations in embayment width. As a consequence, systems characterized by large depth fluctuations may tend to fill more quickly with sediment, whereas systems characterized by large width fluctuations may represent more stable morphologies.

However the study of temporal asymmetries in shallow channelized tidal embayments still lacked a straightforward analytical derivation. Analytic methods, where tractable, have the potential to relate a process to its range of consequences in the most concise manner possible, condensing a wide range of forcings and outcomes into a single expression. But analytic methods are only useful if they both clarify the process of interest and simultaneously retain the most important physics. A theoretical approach can easily retain so many terms that all feeling for the underlying processes is lost. For example, previous analytic studies of tidal asymmetries (e.g., Kreiss 1957; Shetye and 
Gouveia 1992) have employed formal perturbation techniques which result in many terms contributing to each overtide and render physical interpretation difficult.

In Chapter 2 of this thesis (and also in Chapter 4) a simpler perturbation scheme is applied to tidal propagation in shallow embayments. This less formal perturbation in time (but not space) is asymptotically correct for embayments much shorter in length than the tidal wave and maintains the fundamental features of "exact" numerical solutions in longer systems as well. This approach allows all the major geometric features controlling tidal asymmetry to be combined into a single parameter, $\gamma$. The solutions for the overtides which determine tidal asymmetry are scaled by $\gamma$, are compact, and are easily interpreted. For $\gamma>0$, time-varying depth plays a larger role in determining asymmetry than timevarying width and the rising tide is of shorter duration. For $\gamma<0$, time-varying width is more important and the falling tide is of shorter duration.

\subsection{Morphologic ramifications of spatial asymmetries in $\tau$}

The morphologic investigations in this thesis are largely motivated by longstanding models of morphologic equilibrium and net sediment transport in shallow tidal systems based on the spatial distribution of bottom shear stress ( $\tau$ ). Emphasis on spatial asymmetries in $\tau$ actually predates the discussion of temporal asymmetries in the literature. For tidal inlets, Bruun and Gerritsen (1960) and Bruun (1967) developed an equilibrium criterion based on an empirical stability shear stress, $\tau_{s}$. They argued that wherever $\tau<\tau_{s}$, cross-sectional area will be reduced by deposition, and a constriction of flow will then increase $\tau$ back toward $\tau_{s}$. Wherever $\tau>\tau_{s}$, cross-sectional area will be increased by erosion, and an expansion of flow will decrease $\tau$ back toward $\tau_{s}$. The often cited "scour lag" and "settling lag" effects espoused by Postma $(1961 ; 1967)$ are also mechanisms by which sediment moves from areas of high $\tau$ toward areas of low $\tau$. Equilibrium criteria for tidal channels based on a uniform dissipation of energy (Myrick and Leopold 1963; Wright et al. 1973) can also be re-expressed in terms of a uniform shear stress model.

The study of morphologic change and equilibrium based on the spatial distribution of shear stress is a simplification of more a physically grounded but complicated approach based on divergence of sediment flux. Since common formulations for erosion, deposition and net transport are generally expressed as functions of $\tau$, often in the form of power relations (e.g., Dyer 1986), the spatial distribution of $\tau$ is a useful starting point before attempting to estimate sediment transport directly using more uncertain predictions. 
Bottom shear stress can be derived from hydrodynamic relations with a greater degree of confidence.

In Chapter 3 of this thesis, equilibrium models based on uniform $\tau$ are tested by examining spring tidal discharge and cross-sectional geometry from 146 sections in 18 separate tidal systems. Until now, no systematic survey of existing data on the spatial distribution of $\tau$ had been performed. In Chapter 3, the distribution of $\tau$ within individual systems is found to be statistically consistent with uniform $\tau$. The critical shear stress just capable of initiating sediment motion is found to provide a lower bound on $\tau$, and the characteristic value of $\tau$ appropriate to individual systems is found to be a function of spring tidal range. Small along-channel deviations away from uniform $\tau$ are associated with along-channel variation in the direction of maximum discharge, suggesting a feedback mechanism between temporal and spatial asymmetries in $\tau$ : It is hypothesized that convergence in the direction of maximum shear stress (due to temporal asymmetries) causes deposition, a reduction in cross-sectional area, and an increase in velocity. Area is reduced and velocity is increased until a locally increased magnitude of $\tau$ is reached which effectively disperses the sediment once more (via spatial asymmetries).

An analogous study of intertidal flat morphology in Chapter 5 is inspired by success in examining the spatial distribution of $\tau$ along tidal channels. The application of uniform $\tau$ concepts to intertidal flats is further motivated by recent empirical observations of tidal flat morphology (e.g., Dieckmann et al. 1987; Kirby 1992) which have associated characteristic hypsometric forms (the distribution of basin area as a function of elevation) - with different types of hydrodynamic forcing. Hypsometric analysis has the advantage of representing broad aspects of tidal flat morphology in a concise and quantitative manner. Chapter 5 applies uniform $\tau$ to derive equilibrium hypsometries for flats exposed either to tidal currents or to wind waves. Domination of $\tau$ by tidal currents is found to favor convex hypsometries, and domination of $\tau$ by wind waves is found to favor concavity, each in a manner consistent with empirical observations. In addition to the role played by uniform $\tau$, the effect of shoreline curvature on equilibrium hypsometry is found to be important.

\subsection{Basic momentum and mass balances}

Although originally motivated by the mechanisms which control temporal asymmetries in $\tau$, the hydrodynamic investigation in Chapter 2 also illuminates important aspects of basic force balances in shallow tidal channels. A scaling of the governing 
equations for prismatic channels which are characterized by strong tidal asymmetries indicates the friction term in the momentum equation often dominates acceleration at lowest order. Application of "zero-inertia" to short prismatic channels results in a single timevarying diffusion equation which clarifies the most basic dynamics, namely a balance between pressure gradient and friction. The balances applied in Chapter 2 build on the work of LeBlond (1978), who utilized zero-inertia in prismatic channels of infinite length.

Investigation of spatial asymmetries in $\tau$ in Chapter 3 indicates a nearly uniform distribution of $\tau$ is found along many tidal channels and suggests a new scaling of the continuity equation for channels near morphologic equilibrium. This is the primary motivation for the hydrodynamic investigation in Chapter 4 of this thesis. In tidal channels having a nearly uniform distribution of $\tau$, along-channel gradients in velocity are small and discharge gradients in continuity are often dominated by gradients in crosssectional area. In Chapter 4 it is also shown that the above scaling of continuity guarantees a zero-inertia balance in momentum. The result is a first-order wave equation with solutions which are simpler and more easily interpreted than previous solutions for tides in convergent channels with friction.

Previous analytic solutions for the dominant tidal frequency in shallow channels have generally emphasized similarities between solutions with friction and classic frictionless solutions. In textbooks, for example, the governing equation with (weak) friction is typically assumed to be a damped second-order wave equation and the solution is described by nodes and anti-nodes resulting from the interaction of incident and reflected waves (e.g., Ippen 1966; Officer 1976). In Chapters 2 and 4 of this thesis the governing equations and solutions derived for tides in shallow embayments diverge markedly from these classical solutions.

In Chapter 2, the time-varying diffusion equation only permits tidal amplitude to decay along-channel. Thus nodes and anti-nodes cannot occur. In Chapter 4 , the firstorder wave equation results in a constant amplitude waveform which can only propagate forward and is independent of channel length -- in contrast to the length-sensitive resonance of classical co-oscillation. Amplitude can grow or decay along channel if the effects of acceleration and velocity gradients are taken into account at higher order, but the resulting along-channel variations in tidal amplitude are due to variations in the rate of channel convergence rather than interactions between incident and reflected waves. The slight attention previously paid to tides in shallow convergent channels may be due in part 
to the complex nature of the few solutions (Hunt 1964; Jay 1991) which have previously emphasized their unique character.

\subsection{Future work}

This thesis is motivated in large part by the roles of temporal and spatial asymmetries in $\tau$ in determining equilibrium forms and patterns of net sediment transport. This thesis succeeds in quantifying the mechanisms responsible for temporal asymmetries and in deriving forms which will result if spatial asymmetries are minimized. Preliminary feedback mechanisms between these two processes are also identified for some equilibrium tidal channels. However, much work remains to be done in evaluating the applicability and relative importance of these two mechanisms and in synthesizing them in systems where both play a role.

One promising line of research involves a return to diagnostic numerical modeling in the spirit of Speer and Aubrey (1985) and Friedrichs and Aubrey (1988). Future modeling efforts can take advantage of the equilibrium forms derived in this thesis and examine patterns of temporal and spatial asymmetries in more realistic two-dimensional embayments which include flow over intertidal flats and morphologic forms not easily studied by analytic methods. Some initial progress has been made in this area, including the numerical implementation of a zero-inertia balance to solve for intertidal flows in a realistic yet numerically stable fashion (Friedrichs et al. 1990; Friedrichs et al. 1992).

Important questions which can be addressed via diagnostic numerical modeling include: What are the likely patterns of temporal asymmetries in $\tau$ in basins with minimized spatial asymmetries in $\tau$ ? If strong temporal asymmetries in $\tau$ do exist in these more realistic embayments, will the temporal asymmetries cause spatial asymmetries to develop which will create feedback and counteract the effects of the temporal asymmetries? If so, is this tendency true of most realistic basin forms? Or are there specific forms which are likely to evolve towards equilibrium and other forms which are inherently unstable? Preliminary work is underway to address some of these questions (Friedrichs et al. in prep, b).

Another issue that requires further attention is the role of wind waves in generating patterns of bottom shear stress in tidal embayments, especially over intertidal flats. Over many tidal flats, wind waves (rather than tidal currents) can be expected to be the major source of bottom shear stress. The role of wind waves in determining equilibrium flat 
morphology is addressed in a simplified manner in Chapter 5, and a field study is underway to provide some relevant observations (Friedrichs et al. in prep, a). However much work remains to be done, including the addition of waves to some degree in future numerical modeling efforts.

Finally, additional theoretical and observational justification for the morphologic approach employed in this thesis is desired. Morphologic change based on the spatial distribution of $\tau$ assumes dispersive modes of sediment transport are at least as important as other modes which are based on systematic properties of the velocity field (such as temporal asymmetries due to tidal nonlinearities). Unlike temporal asymmetries, the properties of the velocity field which lead to dispersive sediment transport are not well resolved -- although dispersive mechanisms which have been previously applied to the mixing of tracers (e.g., Fischer et al. 1979) likely play a role. 
Chapter 2:

Nonlinear Diffusion of the Tidal Signal in Frictionally Dominated Embayments 


\section{Abstract to Chapter 2}

The dynamics of many shallow tidal embayments may be usefully represented by a single "zero-inertia" equation for tidal elevation which has the form of a nonlinear diffusion equation. The zero-inertia equation clarifies the lowest order dynamics, namely, a balance between pressure gradient and friction. It also provides insight into the properties of higher-order harmonic components via the identification of compact approximate solutions and governing nondimensional parameters.

Approximate analytic solutions which assume a constant diffusion coefficient are governed by the nondimensional parameters $x / L$ and $\left\|k_{0}\right\| L$, where $L$ is the length of the embayment, and $\left\|k_{0}\right\|^{-1}$ scales both the length of frictional dissipation and the physical length of the diffusive waveform. As $\left\|k_{0}\right\| L$ increases, the speed at which the tidal signal diffuses decreases and the rate of decay of tidal amplitude with distance increases. The parameter $\left\|k_{0}\right\| L$ increases as depth is reduced, friction is increased, forcing amplitude or frequency is increased, or total embayment width is increased relative to the width of the channel.

Approximate analytic solutions which assume a time-varying diffusion coefficient result in additional components at the zeroth, second and third harmonic frequencies. The zeroth and second harmonics are governed by the parameter $\gamma$, as well as $x / L$ and $\left\|k_{0}\right\| L$. The parameter $\gamma$ measures the relative importance of time variations of channel depth $(\gamma>$ 0 ) versus time variations in embayment width $(\gamma<0)$. If $\gamma>0$, the diffusion coefficient is larger near the crest of the tidal waveform, causing the rising tide to be of shorter duration and mean elevation to be set up. If $\gamma<0$, the diffusion coefficient is larger near the trough, causing the falling tide to be shorter and elevation to be set down. The third harmonic is produced by fluctuations in the diffusion coefficient associated with times of greatest surface gradient. The third harmonic is governed only by the parameters $x / L$ and $\left\|k_{0}\right\| L$, which indicates the third harmonic is insensitive to time variations in cross-sectional geometry.

Comparisons to field observations and to numerical solutions of the full equations including inertia terms indicate that the zero-inertia equation (1) reproduces the results of the more general one-dimensional equations to within the accuracy predicted by scaling arguments and (2) reproduces the main features of the nonlinear tidal signal observed in many shallow tidal embayments. 
List of symbols in Chapter 2

a amplitude of $\zeta$

$A A$ advective acceleration scale

$A_{m} \quad$ amplitude of $\zeta_{m} / a$

$b$ total embayment width

$b_{0} \quad$ time-average of $b$

$\bar{b}_{0} \quad x$-independent, representative value of $b_{0}$

$b_{c} \quad$ channel width

$\bar{b}_{c} \quad x$-independent, representative value of $b_{c}$

$\bar{b}_{H} \quad S_{H} / L$

$c_{d} \quad$ drag coefficient

$D_{0} \quad$ first-order diffusion coefficient

$F \quad$ friction scale

$g$ acceleration of gravity

$h$ cross-sectionally averaged channel depth

$h_{0} \quad$ time-average of $h$

$\bar{h}_{0} \quad x$-independent, representative value of $h_{0}$

$h_{1}$ elevation of lower break in intertidal slope

$h_{2}$ elevation of upper break in intertidal slope

I inertia scale

$k_{0} \quad$ first-order complex wave number

$L \quad$ total length of tidal channel

$L A \quad$ local acceleration scale

$m \quad$ signifies $m$ th harmonic component

$n$ Manning's friction coefficient

$S_{0} \quad$ time-averaged submerged surface area of embayment

$S_{H} \quad$ submerged surface area of embayment at mean high water

$S_{c} \quad$ submerged surface area of embayment at mean low water

$t$ time

$T \quad$ tidal period

$u \quad$ cross-sectionally averaged velocity

$U$ amplitude of $u$

$x \quad$ along-channel co-ordinate

$\alpha \quad 5 /\left(3 h_{0}\right)$

$\beta \quad(1 / a)\left(\Delta b / b_{0}\right)$ 
List of symbols in Chapter 2 (continued)

$\begin{array}{ll}\Delta b & \text { amplitude of tidal variation in } b \\ \delta & \text { parameter governing third harmonic } \\ \varepsilon & \text { amplitude of tidal variation in }|\partial \zeta \partial x|^{1 / 2} \\ \gamma & \text { parameter governing tidal asymmetry } \\ \zeta & \text { tidal elevation } \\ \zeta_{m} & \text { mth harmonic in } \tau \text {-domain } \\ \eta_{m} & m \text { th harmonic in } t \text {-domain } \\ \theta & 2 \varphi+\pi / 2 \\ \varphi_{m} & \text { phase angle of } \zeta_{m} \\ \xi & x \text {-dependent portion of } \zeta \\ \xi_{m} & x \text {-dependent portion of } \zeta_{m} \\ \tau & \text { transformed time variable } \\ \phi & \text { phase angle of tanh } k_{0} L \\ \omega & \text { tidal frequency }\end{array}$




\subsection{Introduction}

In the study of open channel flow and flood routing, it has long been recognized that the zero-inertia approximation results in a nonlinear diffusive governing equation which advantageously can be applied to gradually varying unsteady problems (Hayami 1951; Henderson 1966; Ponce et al. 1978). Application of the zero-inertia approximation to flood routing leads to depth and storage dependent flood crest propagation and dissipation, and accounts for the highly asymmetric rise and fall typical of flood waves. However, it was not until recently that the zero-inertia approximation was applied to the study of nonlinear flow in tidal channels (LeBlond 1978). LeBlond showed that in shallow tidal rivers, frictional forces exceed inertial forces over most of the tidal cycle. By dropping the inertial terms in the depth-averaged one-dimensional (1-D) momentum equation, he formed a single nonlinear diffusion equation for tidal velocity and showed that long time lags associated with the propagation of low water could be accounted for by the form of the nonlinear diffusion coefficient.

Since the important work of LeBlond (1978), many papers have investigated nonlinearities in frictionally dominated tidal embayments using a combination of scaling arguments, field observations, and numerical modeling (Parker 1984, 1991; Aubrey and Speer 1985; Speer and Aubrey 1985; Friedrichs and Aubrey 1988; Westerink et al. 1989; Münchow and Garvine 1991; Friedrichs et al. 1992). However, the study of tidal propagation in frictionally dominated embayments is still lacking an analytically based discussion of overtides which includes all four principal sources of nonlinearity: quadratic friction, time-varying channel depth in the friction term, and time-varying channel depth coupled with time-varying embayment width in the continuity equation. No second-order analytic study has considered the generation of harmonics by large variations in embayment width during the tidal cycle, which is the primary source of nonlinearity in many tidal embayments of interest (e.g., Boon and Byme 1981; Friedrichs and Aubrey 1988). Through analytic methods, the present paper aims to synthesize all these nonlinear mechanisms in a manner most easily adapted to physical interpretation.

Previous second-order, linearized solutions to the 1-D equations with friction have been found via formal perturbation analyses (Kreiss 1957; Gallagher and Munk 1971; Li 1974; Kabbaj and LeProvost 1980; Uncles 1981; DiLorenzo 1988; Shetye and Gouveia 1992). Although rigorous perturbation expansions are important for spectral modeling of overtides and compound tides (Kabbaj and LeProvost 1980), such techniques can make 
simple physical interpretation of analytical results difficult. When applied to the full 1-D equations for tidal embayments, formal perturbation analysis is algebraically intensive and results in solutions with many terms contributing to each overtide.

In this study we take a less formal approach. We make a zero-inertia assumption along the lines of LeBlond (1978), and form a single nonlinear diffusion equation for tidal elevation. Second-order solutions are found by approximating the nonlinear diffusion coefficient as constant in space and expanding only the time-varying portion. This approach conveniently combines the four primary nonlinear mechanisms into a single time-varying coefficient. Approximate analytic solutions for the zeroth, second, and third harmonic components are compact and allow straightforward physical interpretation via identification of their governing nondimensional parameters. Finally, we compare our approximate analytic solutions to field observations and to "exact" numerical solutions with and without the inertial terms.

In this study we examine the nonlinear properties of tidal elevation in tidal channels closed at one end. This particular application was chosen because of its relevance to a large volume of readily available field observations. Nonlinear tidal velocities in similar channels can also be examined with the 1-D zero-inertia equation. This equation may also be applied to the nonlinear properties of tidal velocity and elevation in channels with elevations forced at either end (e.g., Wong 1989). These topics are the subject of ongoing research.

\subsubsection{The frictional dominance assumption}

Through scaling arguments, field measurement and/or numerical modeling of the individual terms in the 1-D momentum equation, many authors have demonstrated the dominance of friction over the inertial terms in well-mixed, shallow tidal embayments and estuaries. A survey of the literature (Table 2.1) indicates that in systems of interest (wellmixed, tidal amplitude/mean depth $>\sim 0.1$, tidal velocities $\sim 0.5 \mathrm{~m} \mathrm{~s}^{-1}$ ), the friction term is typically 1 to 2 orders of magnitude larger than either the local or the advective acceleration term. Furthermore, the local and advective acceleration terms are typically of opposite sign and partially cancel. In a recent paper, Jay (1991) showed that the acceleration terms can be entirely cancelled to lowest order by topographically generated terms in tidal channels with exponentially convergent geometries. 
The last two entries in Table 2.1 serve to demonstrate the limits of the frictional dominance assumption. In the Lower Columbia River Estuary, where salinity intrusion is present, the shear stress at the bed is reduced by stratification in the water column, partially decoupling the overlying flow from the bottom (Giese and Jay 1989). Upriver beyond the intrusion of salinity, the tidal pressure gradient is primarily balanced by friction (Giese and Jay 1989). In the final example (Pingree and Maddock 1978), the English Channel is simply too deep and bottom stress too small for friction to dominate the momentum equation. Approximate quantitative bounds on the conditions under which friction dominates inertia in well-mixed tidal embayments are provided by a scalar analysis of the 1-D governing equations.

The cross-sectionally integrated, 1-D equations of motion for well-mixed, channelized flow in a tidal embayment with intertidal flats (Figure 2.1) may be expressed as (e.g., Speer and Aubrey 1985)

$$
\begin{gathered}
b \frac{\partial \zeta}{\partial t}+\frac{\partial\left(b_{c} h u\right)}{\partial x}=0 \\
\frac{\partial u}{\partial t}+u \frac{\partial u}{\partial x}+g \frac{\partial \zeta}{\partial x}+\frac{c_{d} u|u|}{h}=0
\end{gathered}
$$

where $b$ is total embayment width (including tidal flats), $\zeta$ is tidal elevation, $h$ is crosssectionally averaged channel depth, $b_{c}$ is the width of the channel, $u$ is cross-sectionally averaged velocity (confined to the channel), and $c_{d}$ is the drag coefficient. In addition to the usual assumptions of channelized flow, (2.1)-(2.2) assume $u=0$ on the tidal flats, and $b_{c} / h \gg 1$.

Restated in terms of characteristic scales, (2.1) and (2.2) become

$$
\begin{gathered}
\frac{b a}{T}+\frac{h b_{c} U}{L}=0, \\
\frac{U}{T}+\frac{U^{2}}{L}+\frac{g a}{L}+\frac{c_{d} U^{2}}{h}=0,
\end{gathered}
$$

where $a$ and $U$ are the amplitudes of tidal elevation and velocity, $T$ is the tidal period, and $L$ is the characteristic horizontal length scale. Here we are assuming that the length scales of variation in $u$ and $\zeta$ are of the same order. Thus this analysis is limited to nonlinearities with a basin-wide character and does not consider advective nonlinearities typically 
localized to. smaller geometric features such as inlets, sand banks, or channel meanders (Zimmerman 1978).

Solving for $L$ in (2.3a) and then eliminating $L$ in (2.3b) gives

$$
\frac{U}{T}+\frac{b a U}{b_{c} h T}+\frac{b g a}{b_{c} h U T}+\frac{c_{d} U^{2}}{h}=0 .
$$

The sum of the inertia terms is $O(U / T)$ if we assume $O\left(b a / b_{c} h\right) \leq 1$. Combining the first two terms of (2.4) and dividing (2.4) by its third term gives the magnitudes of inertia and friction relative to the pressure gradient, which we assume to be order one:

$$
\frac{b_{c} U^{2} h}{b a^{2} g}+1+\frac{b_{c} c_{d} U^{3} T}{b a^{2} g}=0 .
$$

The ratio of the friction scale to the inertial scale is then

$$
\frac{F}{I}=\frac{T U c_{d}}{h} .
$$

In shallow tidal embayments of interest, $U$ is of the order $0.5 \mathrm{~m} \mathrm{~s}^{-1}, c_{d} \approx$ $10^{-2} \cdot 10^{-3}$, the semidiurnal period $T=4.5 \times 10^{4} \mathrm{~s}$, and $1 \mathrm{~m} \leq h \leq 10 \mathrm{~m}$. Therefore $F$ will typically be 1 to 2 orders of magnitude larger than $I$ in these tidally dominated embayments. Since $F / I$ is frequency dependent, however, one should use the period of the overtide of interest when considering highly nonlinear flow. This is not a serious limitation: $F$ will still dominate $I$ by an order of magnitude, even if one scales (2.6) with the quarter-diurnal tidal period.

\subsubsection{Derivation of the zero-inertia equation}

If we assume that frictional effects are much larger than acceleration (i.e., $F / I>>$ 1), then the momentum equation for cross-sectionally averaged flow in a tidal embayment may be expressed, accurate to $\mathrm{O}(F /)^{-1}$, as

$$
g \frac{\partial \zeta}{\partial x}+\frac{n^{2} g u|u|}{h^{4 / 3}}=0,
$$

or, equivalently,

$$
u=-\frac{h^{2 / 3}}{n|\partial \zeta / \partial x|^{1 / 2}} \frac{\partial \zeta}{\partial x}
$$


where $n=h^{1 / 6}\left(c_{d} / g\right)^{1 / 2}$ is Manning's friction coefficient, which is assumed to be constant in space and time. (If using complex notation for $\zeta$, the expression $|\partial \zeta / \partial x|=$ $\operatorname{Abs}\{\operatorname{Re}(\partial \zeta / \partial x)\})$.

Inserting (2.7b) into (2.1) yields a single governing equation for tidal elevation in the form of a nonlinear diffusion equation:

$$
\frac{\partial \zeta}{\partial t}-\frac{1}{b} \frac{\partial}{\partial x}\left\{\frac{b_{c} h^{5 / 3}}{n \mid \partial \zeta \partial x^{1 / 2}} \frac{\partial \zeta}{\partial x}\right\}=0
$$

There are four sources of nonlinearity in (2.1)-(2.2) which contribute to the time variability of the diffusion coefficient in (2.8): time-varying embayment width, $b$, from continuity; time-varying $|\partial \zeta / \partial x|^{1 / 2}$ from quadratic friction; and two contributions to timevarying channel depth, namely, $h^{2 / 3}$ from the depth effect on friction and another power of $h$ from continuity. Equation (2.8) is solved numerically in Section 2.4, where it is compared to numerical solutions to (2.1)-(2.2), to approximate analytic solutions derived in Section 2.3, and to field observations.

To enable approximate analytic solution, we expand the time-varying geometric parameters:

$$
\begin{gathered}
h^{5 / 3}=h_{0}^{5 / 3}\left(1+\frac{\zeta}{h_{0}}\right)^{5 / 3} \approx h_{0}^{5 / 3}(1+\alpha \zeta), \\
b \approx b_{0}(1+\beta \zeta) \\
|\partial \zeta / \partial x|^{1 / 2}=\left(|\partial \zeta / \partial x|^{1 / 2}\right)_{0}(1+\varepsilon(t))
\end{gathered}
$$

where

$$
\alpha=\frac{5}{3 h_{0}}, \quad \beta=\frac{1}{a} \frac{\Delta b}{b_{0}},
$$

and the dependence of $\varepsilon$ on $t$ will be determined in a later section. In (2.9) the subscript zero indicates time-averaged values, and $\Delta b$ is the amplitude of change in $b$ during the tidal cycle. Introducing (2.9a)-(2.9d) in $(2.8)$ yields

$$
\frac{\partial \zeta}{\partial t}-\frac{1}{b_{0}(1+\beta \zeta)} \frac{\partial}{\partial x}\left\{\frac{b_{c} h_{0}^{5 / 3}(1+\alpha \zeta)}{n\left(|\partial \zeta / \partial x|^{1 / 2}\right)_{0}(1+\varepsilon(t))} \frac{\partial \zeta}{\partial x}\right\}=0
$$


In the following sections we develop approximate analytic solutions to (2.10). These analytic approximations allow a straightforward interpretation of the lowest order dynamics and provide insight into the properties of higher-order harmonic components via the identification of their governing nondimensional parameters.

\subsection{Constant diffusion coefficient}

\subsubsection{Solution}

In solving the lowest order case, we neglect terms $\mathrm{O}(\alpha \zeta, \beta \zeta, \varepsilon)$ and assume $b_{0}, b_{c}$, $h_{0}$ and $\left(|\partial \zeta / \partial x|^{1 / 2}\right)_{0}$ may be treated adequately by $x$-independent values. Then $(2.10)$ becomes

$$
\frac{\partial \zeta}{\partial t}-D_{0} \frac{\partial^{2} \zeta}{\partial x^{2}}=0
$$

where

$$
D_{0}=\frac{\bar{b}_{c} \bar{h}_{0}^{5 / 3}}{\bar{b}_{0} n \sqrt{\left(|\partial \zeta / \partial x|^{1 / 2}\right)_{0}}}=\text { constant }
$$

and the overbars indicate $x$-independent, representative values. The boundary conditions for (2.11) are (with the landward end at $x=0$ )

$$
\zeta(x=L)=a \cos \omega t, \quad \frac{\partial \zeta}{\partial x}(x=0)=0 .
$$

It is not necessary to assume $b_{0}, b_{c}$ and $h_{0}$ are $x$-independent to reach a first-order analytic solution. A geometric or exponential dependence on $x$ may be treated via Bessel functions (Prandle and Rahman 1980; Appendix 1 of this thesis) or by a modified Green's law approach (Jay 1991). For the embayments of interest to this study, however, the assumption of a prismatic geometry simplifies the form of the solution while retaining the essential physics.

For a linear, constant-coefficient governing equation with periodic forcing, it is convenient to employ complex variables and assume a solution of the form

$$
\zeta(x, t)=\xi(x) \exp i \omega t
$$


where it is tacitly understood that only the real part of the complex solution is retained. If we insert (2.14) into (2.11) and solve the resulting ordinary differential equation in $\xi(x)$ subject to the boundary conditions given by (2.13), we have the solution

$$
\zeta=a \frac{\cosh k_{0} x}{\cosh k_{0} L} \exp i \omega t
$$

where

$$
k_{0}=\left(\frac{i \omega}{D_{0}}\right)^{1 / 2}=(1+i)\left(\frac{\omega}{2 D_{0}}\right)^{1 / 2}
$$

The cross-sectionally averaged velocity, $u$, is obtained from the continuity equation (2.1) as the real part of

$$
u=-\frac{i \bar{b}_{0} a \omega}{\bar{b}_{c} \bar{h}_{0} k_{0}} \frac{\sinh k_{0} x}{\cosh k_{0} L} \exp i \omega t
$$

\subsubsection{Nature of the constant coefficient solution}

For values of $\left\|k_{0}\right\| L \ll 1\left(\left\|k_{0}\right\|\right.$ is defined as $\left.\left\{\left(\operatorname{Re}\left(k_{0}\right)\right)^{2}+\left(\operatorname{Im}\left(k_{0}\right)\right)^{2}\right\}^{1 / 2}\right)$, sinh $k_{0} x$ $\approx k_{0} x, \cosh k_{0} L=1$, and

$$
\zeta \approx a \cos \omega t, \quad u \approx \frac{a \bar{b}_{0} L \omega}{\bar{b}_{c} \bar{h}_{0}} \frac{x}{L} \sin \omega t,
$$

i.e., corresponding to the simple pumping mode, with peak velocities preceding high and low water by $90^{\circ}$. Similarly, for $\left\|k_{0}\right\| x>1$, $\sinh k_{0} x \approx \cosh k_{0} x \approx(1 / 2) \exp \left(k_{0} x\right)$, and

$$
\begin{gathered}
\zeta \approx a \exp \left\{\frac{\left\|k_{0}\right\|}{2^{1 / 2}}(x-L)\right\} \cos \left\{\frac{\left\|k_{0}\right\|}{2^{1 / 2}}(x-L)+\omega t\right\}, \\
u \approx \frac{a \bar{b}_{0} L \omega}{\bar{b}_{c} \bar{h}_{0}\left\|k_{0}\right\| L} \exp \left\{\frac{\left\|k_{0}\right\|}{2^{1 / 2}}(x-L)\right\} \sin \left\{\frac{\left\|k_{0}\right\|}{2^{1 / 2}}(x-L)+\omega t-\frac{\pi}{4}\right\},
\end{gathered}
$$

i.e., corresponding to an exponentially decaying progressive waveform traveling in the negative $x$ direction, with peak velocities preceding high and low water by $45^{\circ}$. In contrast, a frictionless linear tidal wave in an infinite channel has peak velocities exactly coinciding with extreme water levels.

The nature of the frictionally dominated solution depends strongly on the channel length, $L$, relative to the frictional decay scale, $\left\|k_{0}\right\|^{-1}=\left(D_{0} / \omega\right)^{1 / 2}$ (Figure 2.2), which in turn depends on the value of the diffusion coefficient given by (2.12). To obtain an 
estimate of $D_{0}$, we must evaluate the term $\overline{\left(|\partial \zeta / \partial x|^{1 / 2}\right)_{0}}$. From (2.15), the time-averaged magnitude of $\partial \zeta \partial x$ is

$$
\left|\frac{\partial \zeta}{\partial x}\right|_{0}=\left\|k_{0} a \frac{\sinh k_{0} x}{\cosh k_{0} L}\right\| \frac{2}{\pi}
$$

If we represent (20) with its value at $x=L$, then

$$
\overline{\left.\partial \zeta \partial \partial x\right|_{0}}=\left\|k_{0}\right\| a\left\|\tanh k_{0} L\right\| \frac{2}{\pi} .
$$

Introducing the square root of (2.21) into (2.12), we obtain the necessary closure of the problem, i.e.,

$$
D_{0}=\frac{\bar{b}_{c} \bar{h}_{0}^{5 / 3}}{\bar{b}_{0} n}\left(\left\|k_{0}\right\| a\left\|\tanh k_{0} L\right\| \frac{2}{\pi}\right)^{-1 / 2} .
$$

Since $\left\|k_{0}\right\|=\left(\omega / D_{0}\right)^{1 / 2},(22)$ may be written as a dispersion relationship:

$$
\frac{\left(\left\|k_{0}\right\| L\right)^{3 / 2}}{\left(\left\|\tanh k_{0} L\right\|\right)^{1 / 2}}=\frac{2^{1 / 2} \bar{b}_{0} n a^{1 / 2} \omega L^{3 / 2}}{\pi^{1 / 2} \bar{b}_{c} \bar{h}_{0}^{5 / 3}}
$$

For $\left\|k_{0}\right\| L \ll 1,\left\|\tanh k_{0} L\right\| \approx\left\|k_{0}\right\| L$, and (2.23) reduces to

$$
\left\|k_{0}\right\| L=\left(\frac{\omega}{D_{0}}\right)^{1 / 2} L=\frac{2^{1 / 2} \bar{b}_{0} n a^{1 / 2} \omega L^{3 / 2}}{\pi^{1 / 2} \bar{b}_{c} \bar{h}_{0}^{5 / 3}} .
$$

For $\left\|k_{0}\right\| L \gg 1,\left\|\tanh k_{0} L\right\| \approx 1$, and (2.23) reduces to

$$
\left\|k_{0}\right\| L=\left(\frac{\omega}{D_{0}}\right)^{1 / 2} L=\left\{\frac{2^{1 / 2} \bar{b}_{0} n a^{1 / 2} \omega L^{3 / 2}}{\pi^{1 / 2} \bar{b}_{c} \bar{h}_{0}^{5 / 3}}\right\}^{2 / 3}
$$

According to (2.23)-(2.25), the speed at which the tidal signal diffuses decreases and the rate of decay of tidal amplitude with distance increases as channel depth is reduced, channel length is increased, friction is increased, forcing amplitude is increased, or total embayment width is increased relative to the width of the channel. Equations (2.23)(2.25) also state that the amplitude decay rate increases as frequency is increased, indicating frictionally dominated embayments act as low-pass filters. 


\subsection{Time-varying diffusion coefficient}

\subsubsection{Governing equation}

We now use the results from our constant coefficient solution to estimate the time dependent values of $\left(1+\gamma_{\zeta}\right),(1+\beta \zeta)^{-1}$, and $\left\{\left(|\partial \zeta / \partial x|^{1 / 2}\right)_{0}(1+\varepsilon(t))\right\}^{-1}$, each of which was assumed to be constant in formulating (2.11). We still neglect $x$-dependence in these three expressions, however, and chose values at $x=L$ to be representative. Then from (2.15),

$$
\begin{gathered}
1+\gamma \zeta \approx 1+\gamma a \cos \omega t \\
(1+\beta \zeta)^{-1} \approx(1+\beta a \cos \omega t)^{-1}=1-\beta a \cos \omega t \\
\left\{\left(|\partial \zeta / \partial x|^{1 / 2}\right)_{0}(1+\varepsilon(t))\right\}^{-1} \\
\approx\left\{\left\|k_{0}\right\| a\left\|\tanh k_{0} L\right\||\cos (\omega t+\phi+\pi / 4)|^{-1 / 2}\right.
\end{gathered}
$$

where $\phi=$ the phase angle of $\tanh k_{0} L$, and $\pi / 4=$ the phase angle of $k_{0}$.

Equation (2.26c) may be treated more easily if we consider a Fourier series approximation of $|\cos (\omega t+\phi+\pi / 4)|$ followed by the use the Binomial theorem to approximate the inverse square root:

$$
\begin{aligned}
|\cos (\omega t+\phi+\pi / 4)| & =\frac{2}{\pi}\left\{1+\frac{2}{3} \cos 2(\omega t+\phi+\pi / 4)\right\} \\
|\cos (\omega t+\phi+\pi / 4)|^{-1 / 2} & \\
& \approx\left(\frac{\pi}{2}\right)^{1 / 2}\left\{1+\left(-\frac{1}{2}\right) \frac{2}{3} \cos 2(\omega t+\phi+\pi / 4)\right\}
\end{aligned}
$$

Figure 2.3a compares the left and right hand sides of (2.27b). From Figure $2.3 \mathrm{a}$ we see that the right-hand side of (2.27b) underestimates the value of $|\cos (\omega t+\phi+\pi / 4)|^{-1 / 2}$ at the times when $|\partial \zeta / \partial x|$ is largest, i.e., precisely when we can expect discharge to be greatest and the effects of friction to be most important. Thus we will approximate Icos $\left.(\omega t+\phi+\pi / 4)\right|^{-1 / 2}$ instead as

$$
\begin{aligned}
& |\cos (\omega t+\phi+\pi / 4)|^{-1 / 2} \\
& \quad=\left(\frac{\pi}{2}\right)^{1 / 2}(1+\delta \cos 2(\omega t+\phi+\pi / 4)) \approx\left(\frac{\pi}{2}\right)^{1 / 2}(1-\varepsilon(t)),
\end{aligned}
$$


such that the minima of the two functions coincide exactly (Figure 2.3b). This gives $\delta$ $=(2 / \pi)^{1 / 2}-1 \approx-0.20$. The poles indicated by Figure 2.3 , which are poorly represented by the approximation in (2.28), are not significant because they coincide with slack water when friction is small.

Substituting (2.26) and (2.28) into (2.10) gives

$$
\frac{\partial \zeta}{\partial t}-\left(\frac{\pi}{2}\right)^{1 / 2} \frac{1}{b_{0} n} \frac{(1+\gamma \cos \omega t+\delta \cos (2 \omega t+\theta))}{\left(\left\|k_{0}\right\| a\left\|\tanh k_{0} L\right\|\right)^{1 / 2}} \frac{\partial}{\partial x}\left\{b_{c} h_{0}^{5 / 3} \frac{\partial \zeta}{\partial x}\right\}=0,
$$

where

$$
\begin{gathered}
\theta=2 \phi+\frac{\pi}{2} \\
\gamma=a(\alpha-\beta)=\frac{5}{3} \frac{a}{h_{0}}-\frac{\Delta b}{b_{0}} .
\end{gathered}
$$

Relative to (2.8), (2.29) is accurate to $\mathrm{O}(\gamma, \delta, a \alpha, a \beta)^{2}$ plus an unquantified error due to our choosing $x=L$ to be representative in (2.26). If we once again assume $b_{0}, b_{c}$, and $h_{0}$ to be constant in $x$ then (2.29) reduces to

$$
\frac{\partial \zeta}{\partial t}-(1+\gamma \cos \omega t+\delta \cos (2 \omega t+\theta)) D_{0} \frac{\partial^{2} \zeta}{\partial x^{2}}=0
$$

Bessel functions can be used to find approximate solutions to the higher-order harmonics in embayments with geometrically or exponentially varying along-channel geometry (see Appendix 2). However, the basic physics which determine the properties of the higher-order tidal components in frictionally dominated embayments are more clearly illustrated if we assume a prismatic geometry.

\subsubsection{Solution}

We treat the time-varying portion of the diffusion coefficient in (2.30) by changing variables from $t$ to $\tau$ such that

$$
\frac{\partial \zeta}{\partial t}=\frac{\partial \zeta}{\partial \tau} \frac{\partial \tau}{\partial t}=\frac{\partial \zeta}{\partial \tau}(1+\gamma \cos \omega t+\delta \cos (2 \omega t+\theta))
$$

Then (2.31) becomes

$$
\frac{\partial}{\partial \tau} \zeta(\tau, x)-D_{0} \frac{\partial^{2}}{\partial x^{2}} \zeta(\tau, x)=0
$$


with

$$
\omega \tau=\omega t+\gamma \sin \omega t+\frac{\delta}{2} \sin (2 \omega t+\theta) .
$$

The boundary conditions for (2.32) are still $\partial \zeta / \partial x=0$ at $x=0$, and $\zeta=a \cos \omega t$ at $x=L$. However, the boundary condition at $x=L$ must be transformed to the new time variable, $\tau$. Utilizing (2.33), trigonometric identities, and approximations to $\mathrm{O}(\gamma, \delta, a \alpha, a \beta)^{2}$ (for details, see Appendix 2), it can be shown that

$$
\cos \omega t=\sum_{m=-1}^{3} A_{m} \cos \left(m \omega \tau+\varphi_{m}\right)
$$

where $A_{-1}=-A_{3}=\delta / 4, A_{0}=-A_{2}=\not / 2, A_{1}=1,-\varphi_{.1}=\varphi_{3}=\theta$, and $\varphi_{0}=\varphi_{1}=\varphi_{2}=0$.

Since (2.32) is linear, we may express the solution as a sum of terms $\zeta_{m}$, each satisfying the governing equation

$$
\frac{\partial \zeta_{m}}{\partial \tau}-D_{0} \frac{\partial^{2} \zeta_{m}}{\partial x^{2}}=0
$$

and the boundary conditions

$$
\frac{\partial \zeta_{m}}{\partial x}=0 \text { at } x=0, \quad \zeta_{m}=a A_{m} \cos \left(m \omega \tau+\varphi_{m}\right) \text { at } x=L .
$$

We look for solutions to (2.35) of the form

$$
\zeta_{m}(x, \tau)=a A_{m} \xi_{m}(x) \exp i\left(m \omega \tau+\varphi_{m}\right) .
$$

For $m \neq 1,(2.37)$ is already $\mathrm{O}(\gamma, \delta, a \alpha, a \beta)$, so if we discard $\mathrm{O}(\gamma, \delta, a \alpha, a \beta)^{2}$ terms, (2.37) transforms directly back to

$$
\zeta_{m}(x, t)=a A_{m} \xi_{m}(x) \exp i\left(m \omega t+\varphi_{m}\right) .
$$

In order to transform the $m=1$ case, we must reexpress exp $i \omega \tau$ in terms of $t$. Utilizing (2.33), trigonometric identities, and neglecting $\mathrm{O}(\gamma, \delta, a \alpha, a \beta)^{2}$ terms (for details, see Appendix 2), it can be shown that

$$
\exp i \omega \tau=\exp i \omega t-\sum_{m \neq 1} A_{m} \exp i\left(m \omega t+\varphi_{m}\right) .
$$


Equation (2.39) is substituted into (2.37) for the $m=1$ case, and then the resulting equation is added to (2.38) to reconstruct the full solution in $t$.

We represent the full solution as a sum of single frequency components:

$$
\zeta(x, t)=a \sum_{m=-1}^{3} \eta_{m}(x) \exp i\left(m \omega t+\varphi_{m}\right),
$$

with

$$
\begin{gathered}
\eta_{-1}=\frac{\delta}{4}\left(\xi_{-1}-\xi_{1}\right), \\
\eta_{0}=\frac{\gamma}{2}\left(\xi_{0}-\xi_{1}\right), \\
\eta_{1}=\xi_{1}, \\
\eta_{2}=\frac{\gamma}{2}\left(\xi_{1}-\xi_{2}\right), \\
\eta_{3}=\frac{\delta}{4}\left(\xi_{1}-\xi_{3}\right) .
\end{gathered}
$$

By substituting (2.37) into (2.35) we see that the governing equations for $\xi_{m}(x)$ are

$$
i m \omega \xi_{m}-D_{0} \frac{\mathrm{d}^{2} \xi_{m}}{\mathrm{~d} x^{2}}=0
$$

with boundary conditions

$$
\frac{\mathrm{d} \xi_{m}}{\mathrm{~d} x}=0 \text { at } x=0, \quad \xi_{m}=1 \quad \text { at } x=L .
$$

Equations (2.42)-(2.43) have a solution of the same form as the constant coefficient case:

$$
\xi_{m}=\frac{\cosh x\left(i m \omega / D_{0}\right)^{1 / 2}}{\cosh L\left(i m \omega / D_{0}\right)^{1 / 2}}=\frac{\cosh m^{1 / 2} k_{0} x}{\cosh m^{1 / 2} k_{0} L} .
$$

\subsubsection{Nature of the time-varying coefficient solution}

The harmonics produced by the time-varying coefficient solution are scaled by the nondimensional parameters $\gamma, \delta,\left\|k_{0}\right\| L$, and $x / L$. The parameter $\gamma$ scales the zeroth harmonic, which determines set up or set down, as well as the second harmonic, which 
determines duration asymmetry in the rising and falling tides. If $\gamma$ is positive, there is set up of mean elevation and the embayment is "shorter-rising" (Figure 2.4a). If $\gamma$ is negative, there is set down and the embayment is "shorter-falling" (Figure 2.4b). These effects may be understood physically if we reexamine the definition of $\gamma$ and the relevant governing equation:

$$
\begin{gathered}
\gamma=a(\alpha-\beta)=\frac{5 a}{3 \bar{h}_{0}}-\frac{\Delta \bar{b}}{\bar{b}_{0}} \\
\frac{\partial \zeta}{\partial t}-(1+\gamma \cos \omega t+\delta \cos (2 \omega t+\theta)) D_{0} \frac{\partial^{2} \zeta}{\partial x^{2}}=0 .
\end{gathered}
$$

If $\gamma>0,(2.45)$ indicates changes in channel depth during the tidal cycle are more important than changes in embayment width. (The total effect of time-varying channel depth is, in turn, $2 / 5$ due to nonlinear friction and $3 / 5$ due to nonlinear continuity. These proportions follow from the binomial expansion used to derive $\alpha$ in (2.9).) With $\gamma>0$, the time-varying diffusion coefficient in (2.46) is larger than $D_{0}$ near the crest of the waveform $(\omega t \approx 0)$, when channel depth is greatest. And the diffusion coefficient is smaller than $D_{0}$ near the trough of the waveform $(\omega t \approx \pi)$, when the channel is shallowest. Since the speed at which the waveform diffuses is proportional to the square root of the diffusion coefficient, with $\gamma>0$ the crest diffuses landward faster than the trough, "catching-up" with the trough and causing a shorter-rising asymmetry. Since the rate of decay of the waveform with distance is also proportional to the square root of the diffusion coefficient, with $\gamma>0$ the amplitude of the crest decays more slowly than that of the trough, resulting in set up (Figure 2.4a).

The effect of $\gamma<0$ is simply the opposite of $\gamma>0$. If $\gamma<0$, (2.45) indicates changes in embayment width are more important than changes in channel depth. With $\gamma<$ 0 , the diffusion coefficient in (2.46) is larger than $D_{0}$ near the trough of the waveform ( $\omega t$ $\approx \pi$ ), when the embayment is narrowest, and the diffusion coefficient is smaller than $D_{0}$ near the crest $(\omega t \approx 0)$, when the embayment is widest. Thus with $\gamma<0$, the trough diffuses landward faster then the crest, causing a shorter-falling asymmetry, and the trough decays more slowly than the crest, resulting in set down (Figure 2.4b).

With $\gamma$ held constant, duration asymmetry and set up or down increase as $\left\|k_{0}\right\| L$ is increased or $x / L$ is decreased (with $x=0$ landward) (Figure 2.5). This is a straightforward consequence of the different diffusion speeds and decay rates of the crest and trough of the waveform. As $\left\|k_{0}\right\| L$ increases or $x / L$ decreases, the effective distance 
over which the signals travel increases. Therefore the difference between the crest and trough travel times and the difference between the degree of crest and trough amplitude decay both increase.

These approximate analytic results are consistent with the numerical experiments of Speer and Aubrey (1985). Through finite difference solutions of (2.1)-(2.2), they found that embayments with large tidal amplitude to depth ratios and small areas of intertidal flats tend to be shorter-rising, whereas embayments with small amplitude to depth ratios and large areas of intertidal flats tend to be shorter-falling. Speer and Aubrey also found tidal asymmetry to be more sensitive to channel depth than intertidal flat extent. This latter finding is also consistent with $(2.45)$, which weights $a / \bar{h}_{0}$ more heavily than $\Delta b / \bar{b}_{0}$ in the definition of $\gamma$.

The parameter $\delta$ scales the third harmonic as well as a transfer of some energy back to the first harmonic via (2.41a). The effect of $\delta$ in (2.46) can be understood if we recall that $\theta$ is ultimately related to the phase of the surface gradient. $\delta$ and $\theta$ cause the time-varying diffusion coefficient in $(2.46)$ to be smaller when the surface gradient (i.e., velocity) is largest. In other words, large velocities impede the diffusion of the tidal signal.

The third harmonic does not contribute to duration asymmetries. Unlike $\gamma, \delta$ is not a function of cross-sectional geometry, but, to our order of approximation, $\delta$ is constant for all cross-sections. According to the approximate analytic solutions, the third harmonic varies only as a function of $x / L$ and $\left\|k_{0}\right\| L$. Thus the third harmonic is less sensitive than the second harmonic to time variations in channel cross-section. We should expect the magnitude of the third harmonic to become progressively smaller relative to the zeroth and second harmonics as the overall tidal signal displays stronger duration asymmetry.

\subsection{Comparison to numerical solutions and observations}

\subsubsection{Methods}

Forcing $\mathrm{M}_{2}$ amplitude and geometric parameters required as inputs to the numerical and approximate analytic models are listed in Table 2.2 for 12 tidal embayments on the Atlantic Coast of the United States. The geometric parameters in Table 2.2 were determined by fitting the hypsometry of each embayment to an idealized, prismatic geometry with a cross-section of the form given in Figure 2.1. $S_{c}$ is the horizontal area of the embayment that is submerged at mean low water; $S_{H}$ is the area submerged at mean 
high water, and $S_{0}$ is the time-averaged area. Model widths were determined by averaging these areas over the length of the each real embayment. The parameter $\bar{h}_{0}$ is the spatially averaged depth at mean sea level of the portion of the embayment encompassed by $S_{c}$. The heights $h_{1}$ and $h_{2}$ were chosen to best represent the hypsometry of each embayment using prismatic, linearly sloping intertidal storage areas (see Figure 2.1).

Once prismatic approximations of the twelve real embayments were constructed, finite difference representations of (2.1)-(2.2), which include the inertia terms, were solved for each embayment. Manning's $n$ was the only independently adjustable parameter, and it was varied until the solutions of (2.1)-(2.2) were reasonably consistent with the observed tides (Figure 2.6). For several of the embayments there is significant disagreement between observed and calculated $\mathrm{M}_{2}$ phase lag (Table 2.3; Figure 2.6b), especially for small phase lags. This is largely due to the varied locations, relative to the embayment inlets, of the outside, "forcing" tide gauges needed to calculate the observed phase lags within the embayments. Set up, $M_{2}, M_{4}$ and $M_{6}$ were determined by least squares harmonic analyses of both the observed surface elevations and the numerical solutions to (2.1)-(2.2). Results of the harmonic analyses appear in Table 2.3. Also included in Table 2.3 are analyses of numerical solutions to (2.8), the governing equation without the inertia terms and approximate analytic solutions to (2.8) given by (2.40)(2.41) and (2.44). These solutions were calculated with the same $n$ used in the solution of (2.1)-(2.2).

The embayments at Chatham, which is shorter-rising, and North Inlet, which is shorter-falling, were examined in particular detail. These systems each contain many tide gauges and provide case studies for along-channel variation in tidal distortion.

\subsubsection{Numerical solutions}

Numerical solutions to (2.1)-(2.2) and to (2.8), the equations of motion with and without inertia, are consistent within the scaling arguments presented in Section 2.1.1. As predicted by (2.6), the two numerical solutions for $\mathrm{M}_{2}$ disagree by about $5 \%$ or less, while amplitudes of $M_{4}$ and $M_{6}$ disagree by about $10 \%$ and $15 \%$, respectively (Table 2.3; Figures $2.7 \mathrm{a}, 2.7 \mathrm{c}$, and 2.7e). Phases predicted by the two solutions for each tidal component disagree by only a few degrees (Table 2.3; Figures $2.7 \mathrm{~b}, 2.7 \mathrm{~d}$, and $2.7 \mathrm{f}$ ). Disagreements between the two numerical solutions are largest for embayments with relatively deep channels (e.g., Wachapreague, Price), which is also consistent with (2.6). Nonetheless, these relatively small disagreements do not affect the basic dynamic balance. 
Thus, to the degree that the zero-inertia equation clarifies the fundamental physical balance while maintaining the most important nonlinear processes, the zero-inertia equation is a valid approximation of the more classical 1-D equations typically applied to tidally dominated shallow embayments.

The consistency of the approximate analytic solutions and the "exact" numerical results is quite good. The residuals in Figure 2.7 are all small in comparison to the range of the signal. Of course, there are also important differences between the analytic and numerical solutions. This is not surprising given that $a \alpha=5 a / 3 \bar{h}_{0}$ and $a \beta=\Delta b / \bar{b}_{0}$, which were assumed to be small, actually approach unity in several of the embayments of interest (Table 2.2). There are also some systematic, $x$-dependent differences between the numerical solutions and analytic approximations which are illustrated by a closer examination of the solutions for Chatham and North Inlet (Figure 2.8). Relative to the numerical results, the approximate analytic solutions for $\mathrm{M}_{2}$ (Figures $2.8 \mathrm{a}-2.8 \mathrm{~b}$ ) tend to underestimate both amplitude decay and phase lag for large $x / L$ (i.e., near the forced end) and overestimate them at small $x / L$ (i.e., near the landward end). These discrepancies partly result from our treatment of $|\partial \zeta / \partial x|^{-1 / 2}$ in evaluating (2.8) analytically.

By approximating $|\partial \zeta \partial x|^{-1 / 2}$ as $x$-independent in our analytic solution, we neglect two specific aspects of the fully nonlinear, $x$-dependent problem. First, we do not recover a factor of $1 / 2$ that would appear if we were to expand (2.8) by differentiating an $x$ dependent $|\partial \zeta \partial x|^{-1 / 2}$ :

$$
\left.\left.\frac{\partial}{\partial x}|| \frac{\partial \zeta}{\partial x}\right|^{-1 / 2} \frac{\partial \zeta}{\partial x}\right\}=\operatorname{sign}\left\{\frac{\partial \zeta}{\partial x}\right\} \frac{\partial}{\partial x}\left\{\left|\frac{\partial \zeta}{\partial x}\right|^{1 / 2}\right\}=\frac{1}{2}\left|\frac{\partial \zeta}{\partial x}\right|^{-1 / 2} \frac{\partial^{2} \zeta}{\partial x^{2}} .
$$

Neglecting this differentiation overestimates the diffusion coefficient in both (2.11) and (2.46) and, therefore, underestimates the decay and delay of the tide. (We also tried differentiating $|\partial \zeta \partial x|^{-1 / 2}$ before treating it as $x$-independent, i.e., by including the factor of $1 / 2$. However that equally arbitrary approach caused the approximate analytic solution to be too dissipative in comparison to the numerical solutions. Hence we chose to treat $|\partial \zeta \partial x|^{-1 / 2}$ as $x$-independent throughout the derivation.)

The second error resulting from our treatment of $\mid \partial \zeta \partial x^{-1 / 2}$ relates to the no-flow boundary condition, $\partial \zeta / \partial x=0$, at $x=0$. In (2.21) and (2.26c) we approximate $\partial \zeta / \partial x$ for all $x$ with its nonzero value at $x=L$, therefore underestimating $|\partial \zeta / \partial x|^{-1 / 2}$ at small $x / L$ (where the no-flow condition requires the surface gradient to approach zero). Since 
(2.12) indicates that the diffusion coefficient is proportional to $|\partial \zeta / \partial x|^{-1 / 2}$, at small $x / L$ our approach underestimates the magnitude of the diffusion coefficient and overestimates the decay and delay of the tide. This effect, together with that described in the previous paragraph, causes the decay and delay of the tidal signal to be somewhat too small near the seaward end of the embayment and somewhat too large near the landward end. The nature of discrepancies in the higher-order harmonics is analogous. For example, the approximate analytic solutions for $A_{\mathrm{M} 4} / A_{\mathrm{M} 2}$ (Figure 2.8c) underestimate the transfer of energy to $\mathrm{M}_{4}$ at.large $x / L$ (cf. an underestimate of $\mathrm{M}_{2}$ decay) and overestimate the transfer at small $x / L$ (cf. an overestimate of $M_{2}$ decay).

\subsubsection{Observations}

For the $\mathrm{M}_{2}$ tide, the consistency of the analytic results with the observations (Figures 2.8a-2.8b, and 2.9a-2.9b) supports the overriding importance of just two nondimensional parameters, $\left\|k_{0}\right\| L$ and $x / L$, in determining the degree of amplitude decay and phase lag throughout frictionally dominated tidal embayments. For example, Chatham has a significantly larger value of $\left\|k_{0}\right\| L$ than North Inlet and a correspondingly larger decay and delay of the $\mathrm{M}_{2}$ tide. In both embayments, amplitude decay and phase lag increase landward with decreased $x / L$. Observations are also consistent with the analytically derived roles of $\gamma,\left\|k_{0}\right\| L$ and $x / L$ in determining the amplitude and relative phase of $\mathrm{M}_{4}$ (Figures 2.8c-2.8d, 2.9c-2.9d). Chatham has a large $\left\|k_{0}\right\| L, \gamma>0$, and a large $\mathrm{M}_{4}$; North Inlet has a smaller $\left\|k_{0}\right\| L, \gamma<0$, and a smaller $\mathrm{M}_{4}$. In both embayments, $A_{\mathrm{M} 4} / A_{\mathrm{M} 2}$ increases in magnitude landward toward $x / L=0$. In general, all the embayments with $\gamma>0$ are observed to be shorter-rising $\left(0^{\circ}<2 \varphi_{\mathrm{M} 2}-\varphi_{\mathrm{M} 4}<180^{\circ}\right)$, and all those with $\gamma<0$ are observed to be shorter-falling $\left(180^{\circ}<2 \varphi_{\mathrm{M} 2}-\varphi_{\mathrm{M} 4}<360^{\circ}\right)$. Regardless

of the sign of $\gamma$, observations indicate $A_{\mathrm{M} 4} / A_{\mathrm{M} 2}$ increases as the absolute value of $\gamma$ increases (Table 2.3).

The observed and analytic $A_{\mathrm{M} 6} / A_{\mathrm{M} 2}$ ratios are of the same order, and both tend to increase landward (Table 2.3 ). Observed and analytic $M_{6}$ relative phases $\left(3 \varphi_{\mathrm{M} 2}-\varphi_{\mathrm{M} 6}\right)$ also increase as one moves landward toward $x / L=0$. There is no discernible relationship between time variations in cross-sectional geometry and the observed $M_{6}$ tide, which is consistent with our derivation of a constant governing parameter, $\delta$. The inability of the analytic (or numerical) results to better reproduce the observed $M_{6}$ tide may result from our treatment of Manning's $n$ as constant in space and time. Several field studies of shallow tidal embayments suggest $n$ can be a complex function of tidal height, flow 
direction, and observation location within a single embayment (e.g., Swift and Brown 1983; Wallis and Knight 1984; Lewis and Lewis 1987).

The approximate analytic solutions and numerical results both predict significant set ups of mean tidal elevation in several of the shorter-rising embayments (Table 2.3). Unfortunately, none of the tidal observations for shorter-rising embayments listed in Table 2.2 includes references to an absolute vertical datum. However, set up has been documented in shorter-rising tidal rivers in Great Britain with tidal amplitude to depth ratios too large to be represented by the approximation $(a \alpha)^{2}=\left(5 a / 3 h_{0}\right)^{2}<<1$ employed in this study, e.g., The Fleet (Robinson et al. 1983) and the Conwy (Wallis and Knight 1984), each with $(a \alpha)^{2}=1.8$. Observations from North Inlet do include elevation relative to an absolute datum and suggest a significant set down of the tide within North Inlet (Nummedal and Humphries 1978). Although the approximate analytic solutions to (2.11) do predict a small set down for North Inlet (Table 2.3), the numerical solutions predict a small set up. Perhaps the observed set down is due to non-tidal dynamics or an aspect of the geometry not captured in our prismatic approximation.

\subsection{Summary and conclusions}

Scaling of the 1-D equations indicates that the friction term is typically 1 to 2 orders of magnitude larger than the inertial terms over the range of geometric and hydrodynamic parameters common to many shallow tidal embayments. Neglecting the inertial terms leads to a single "zero-inertia" governing equation for tidal elevation which has the form of a nonlinear diffusion equation. The zero-inertia equation clarifies the fundamental physical balance typical to shallow tidal embayments, while retaining the principal sources of basin-wide nonlinearity, namely, quadratic friction, time-varying channel depth, and time-varying embayment width.

First-order solutions are found by assuming the diffusion coefficient to be constant in both time and space. The first-order solutions are governed by two nondimensional parameters, $\left\|k_{0}\right\| L$ and $x / L$, where $L$ is the length of the embayment, and $\left\|k_{0}\right\|^{-1}$, which is proportional to the square root of the diffusion coefficient, scales both the length of frictional dissipation and the physical length of the diffusive waveform.

As $\left\|k_{0}\right\| L$ increases, the speed at which the tidal signal diffuses decreases and the rate of decay of tidal amplitude with distance increases. For $\left\|k_{0}\right\| L \ll 1$, the solution reduces to a simple pumping mode, whereas for $\left\|k_{0}\right\| L \gg 1$, the solution reduces to an 
exponentially decaying, progressive waveform. $\left\|k_{0}\right\| L$ increases as depth is reduced, friction is increased, forcing amplitude or frequency is increased, or total embayment width is increased relative to the width of the channel.

Second-order solutions are found by approximating the nonlinear diffusion coefficient as constant in space and expanding only the time-varying portion. This. approach conveniently combines the primary nonlinear mechanisms into a single timevarying coefficient. Approximate analytic solutions for the zeroth, second and third harmonic components are compact relative to more formal perturbation analyses and are more easily adapted to physical interpretation.

The zeroth harmonic, which determines set up or down, and the second harmonic, which determines duration asymmetry, are both governed by the parameters $x / L,\left\|k_{0}\right\| L$, and $\gamma=5 a / 3 h_{0}-\Delta b / b_{0}$, where $a$ is forcing amplitude, $h_{0}$ is average channel depth, $\Delta b$ is the amplitude of time variation in embayment width, and $b_{0}$ is average embayment width.

If $\gamma>0$, then time variations in channel depth are more important than time variations in embayment width. With $\gamma>0$, the diffusion coefficient is larger near the crest of the waveform than near the trough. The crest diffuses landward faster and decays slower than the trough, resulting in a shorter-rising asymmetry and set up of mean elevation. If $\gamma<0$, variations in width are more important than variations in depth. With $\gamma<0$, the diffusion coefficient is larger near the trough of the waveform, the trough diffuses faster and decays slower, and the tide is shorter-falling and set down.

The third harmonic is produced by fluctuations in the diffusion coefficient associated with times of greatest surface gradient. The only independent parameters governing the third harmonic response are $\left\|k_{0}\right\| L$ and $x / L$. Thus analytic results indicate the response of the third harmonic is less geometry dependent then the response of the zeroth or second harmonics.

"Exact" numerical solutions show that the zero-inertia equation reproduces the results of the more general 1-D equations, including harmonic overtides, to within the accuracy predicted by scaling arguments for shallow tidal embayments. The approximate analytic solution to the zero-inertia equation also reproduces the main features of the numerical solutions, including the fundamental behavior of $\mathrm{M}_{4}$ and $\mathrm{M}_{6}$. Disagreements between analytic and numerical solutions are largely due to the neglect of space dependence in the diffusion coefficient of the analytic solution. Nonetheless, the insight 
provided into the numerical solutions by the analytic approximations demonstrates the usefulness of the simplified second-order approach.

Finally, observations are also consistent with the analytically derived roles of $\gamma$, $\left\|k_{0}\right\| L$ and $x / L$ in determining the amplitude and relative phase of $M_{2}, M_{4}$ and $M_{6}$. Observed $\mathrm{M}_{2}$ amplitude decay and phase lag generally increase with increased $\| k_{0} \mid L$ or decreased $x / L$ (i.e., landward). All observed embayments with with $\gamma>0$ have shorter rising tides, and all those with $\gamma<0$ have shorter falling tides. Observations also indicate the amplitude of $\mathrm{M}_{4}$ generally increases as $\left\|k_{0}\right\| L$ increases, $x / L$ decreases, or the absolute value of $\gamma$ increases. The order of magnitude of the observed $M_{6}$ tide is also reproduced, but observations indicate a significant, unexplained spatial variance that is speculated to result from unresolved temporal and/or spatial variations in real embayment friction factors. 


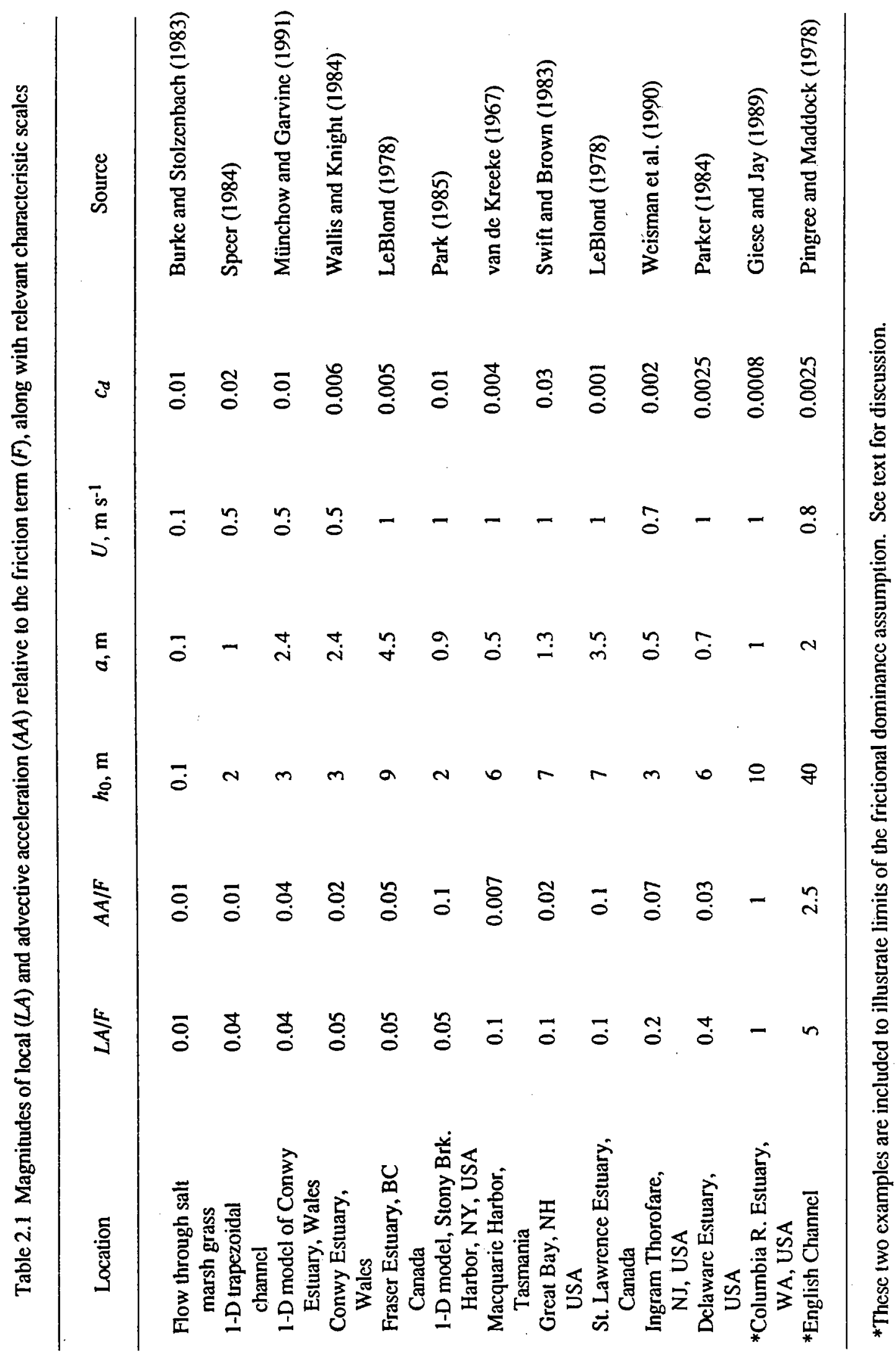



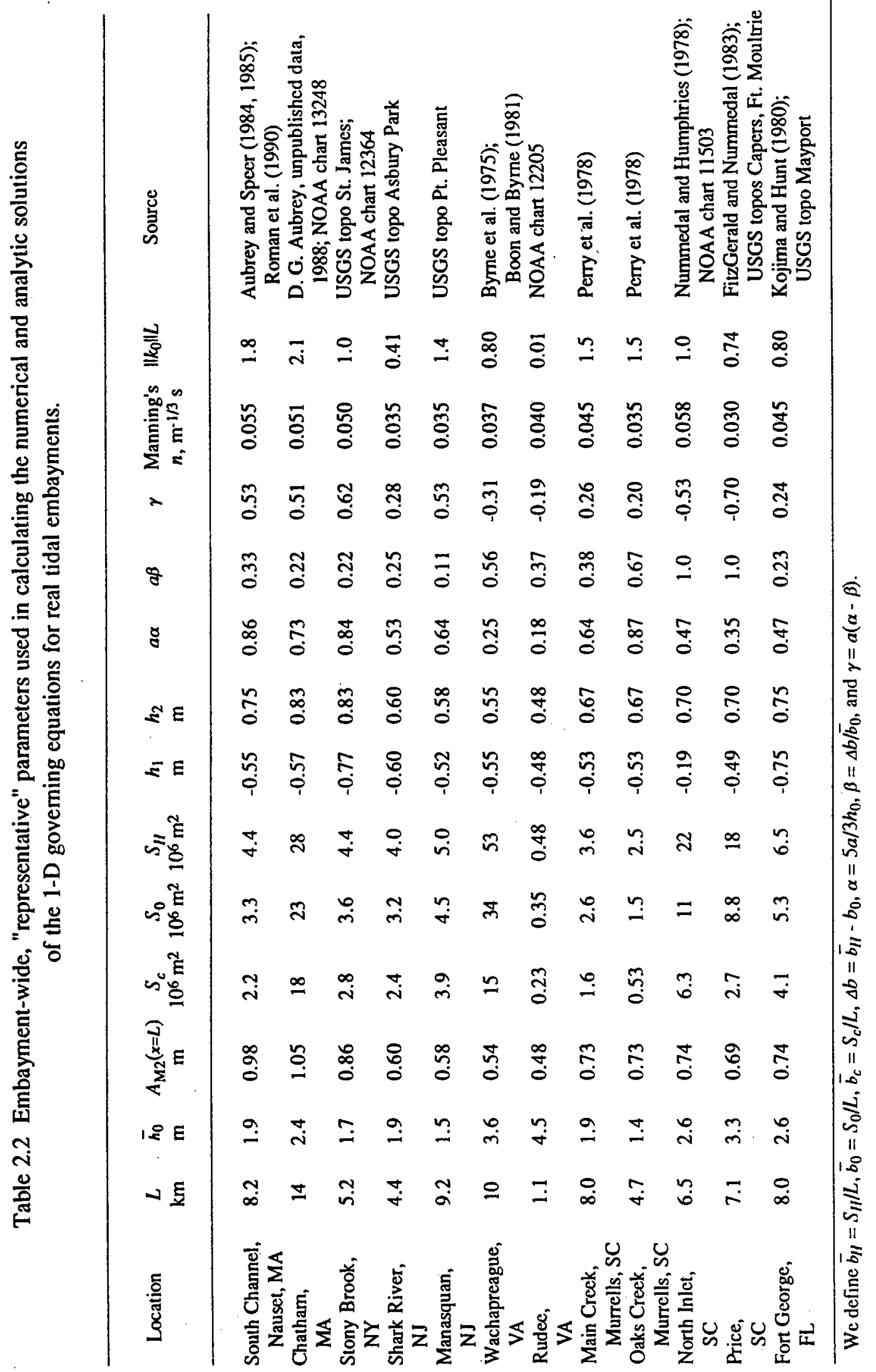


\begin{tabular}{|c|c|c|c|c|c|c|c|}
\hline & २ & 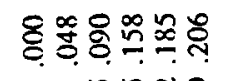 & 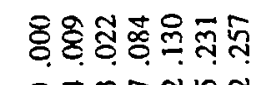 & 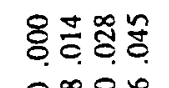 & 8 & 8 & 85 \\
\hline & E्: & 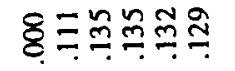 & 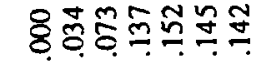 & 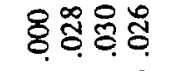 & $\overline{8}$ & 8 & $8 \%$ \\
\hline$\stackrel{\mathscr{c}}{\frac{5}{7}}$ & 咅 $\bar{z}$ & 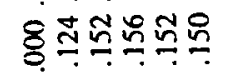 & 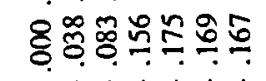 & ఫ్ర ซิర్ర & $\widetilde{్}$ & $8 \overline{8}$ & 8 \\
\hline$\overline{\mathscr{c}}$ & \% & $8:$ & \&ి: & 8 & 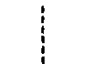 & 8 & 8 \\
\hline 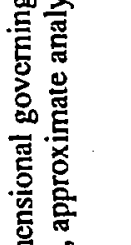 & 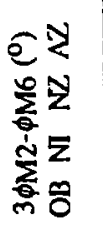 & 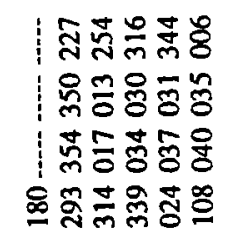 & 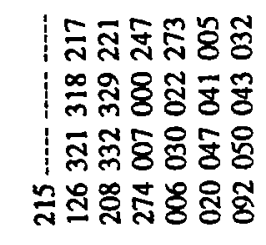 & 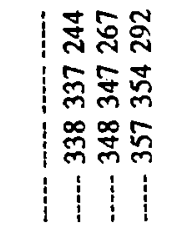 & 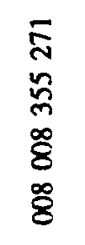 & 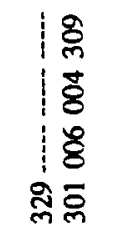 & $\begin{array}{l}\overline{8} \\
\bar{E} \\
\bar{m}\end{array}$ \\
\hline & ע & 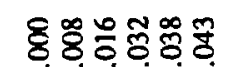 & 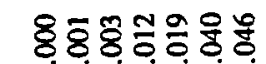 & 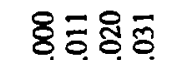 & 8 & 88 & $8 \%$ \\
\hline 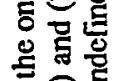 & $\sum_{\Sigma}^{N}$ & 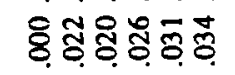 & 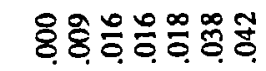 & 8 \&̊ํㅇ음 & 응 & 8 छे & 8 \\
\hline & 文z & రి & 80 & 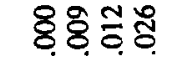 & $\frac{0}{0}$ & 8.8 & 8.5 \\
\hline & $\approx$ & 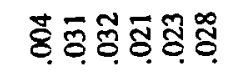 & 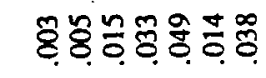 & & $\tilde{\delta}$ & జึ ర్రి & $\overline{0}$ \\
\hline 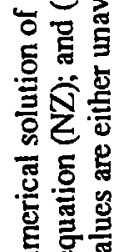 & 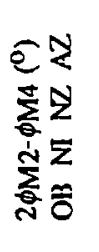 & 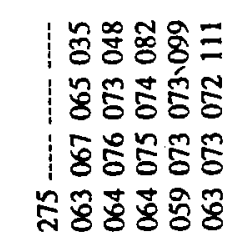 & 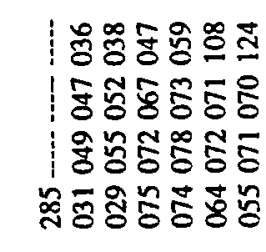 & 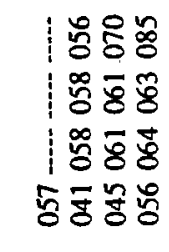 & 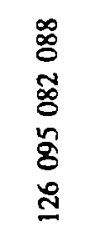 & \begin{tabular}{l}
$n$ \\
0 \\
0 \\
8 \\
8 \\
$\infty$ \\
0 \\
0 \\
$\vdots$ \\
\hdashline
\end{tabular} & \\
\hline 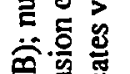 & 8 & 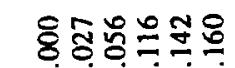 & ఫి ఫిరి & 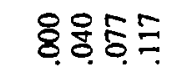 & 응 & 8 & 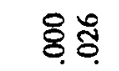 \\
\hline & $\sum_{<}^{T} \Sigma$ & 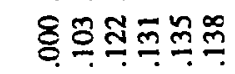 & 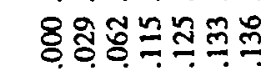 & 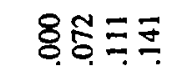 & $\frac{0}{0}$ & 8 & $8 \tilde{8}$ \\
\hline & $\sum_{j}^{\bar{j}} \bar{z}$ & 8 & 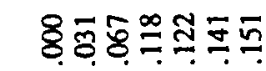 & $8 \div$ & $\stackrel{\infty}{\varrho}$ & $8 \bar{n}$ & 8 \\
\hline 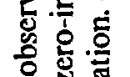 & $\infty$ & $800 \%$ & 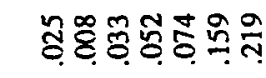 & 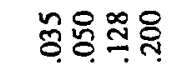 & $\stackrel{\infty}{0}$ & 눙항. & ज̃ \\
\hline 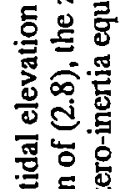 & $\begin{array}{l}\text { à } \\
\sum_{0}^{T} \sum_{0}^{2}\end{array}$ & 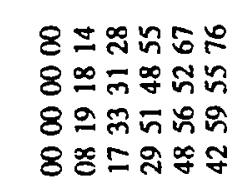 & 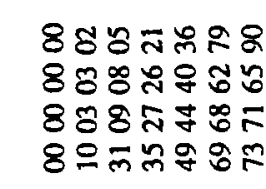 & 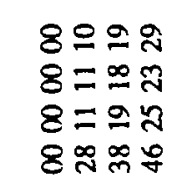 & $\begin{array}{l}n \\
\delta \\
\delta \\
\delta \\
1\end{array}$ & $\begin{array}{l}8 \% \\
8 \% \\
8 \% \\
81\end{array}$ & $\begin{array}{l}8= \\
8 \cong \\
89\end{array}$ \\
\hline 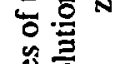 & Z & 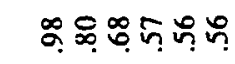 & 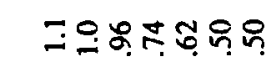 & $?$ & 8 & & ถูก \\
\hline & $\widehat{\underline{E}} \underline{2}$ & 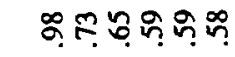 & $=$ ○ం & か゚゚゚ & 8 & ฑొฯ & $\bar{n} \bar{n}$ \\
\hline & $\sum_{k}^{N} \bar{z}$ & 象 & 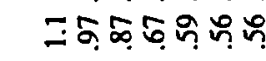 & ๖?ำ & 8 & ฑุษ & $n$ \\
\hline & \% & 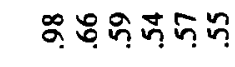 & 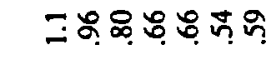 & 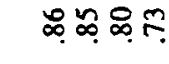 & 8 & 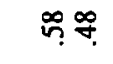 & $n$ \\
\hline E्ञ & $\frac{1}{x}$ & - & - & $-\infty \overline{0}$ & $\stackrel{\circ}{n}$ & $-\check{n}$ & 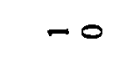 \\
\hline$\cong \tilde{E}$ & 80 & 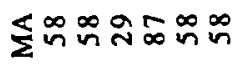 & 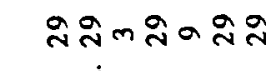 & 욤ni & aิ & $\stackrel{\infty}{\circ}$ & \\
\hline 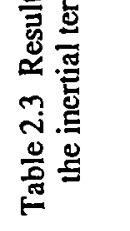 & 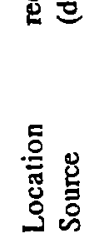 & 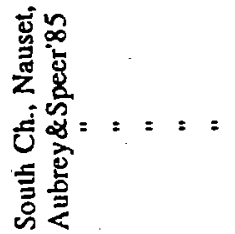 & 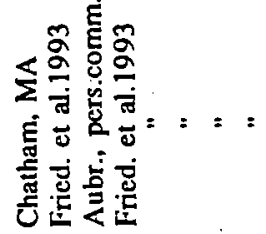 & 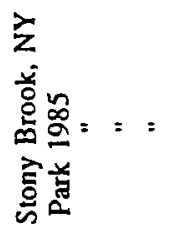 & 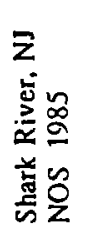 & 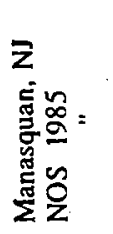 & 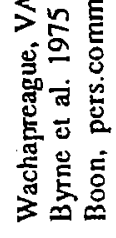 \\
\hline
\end{tabular}




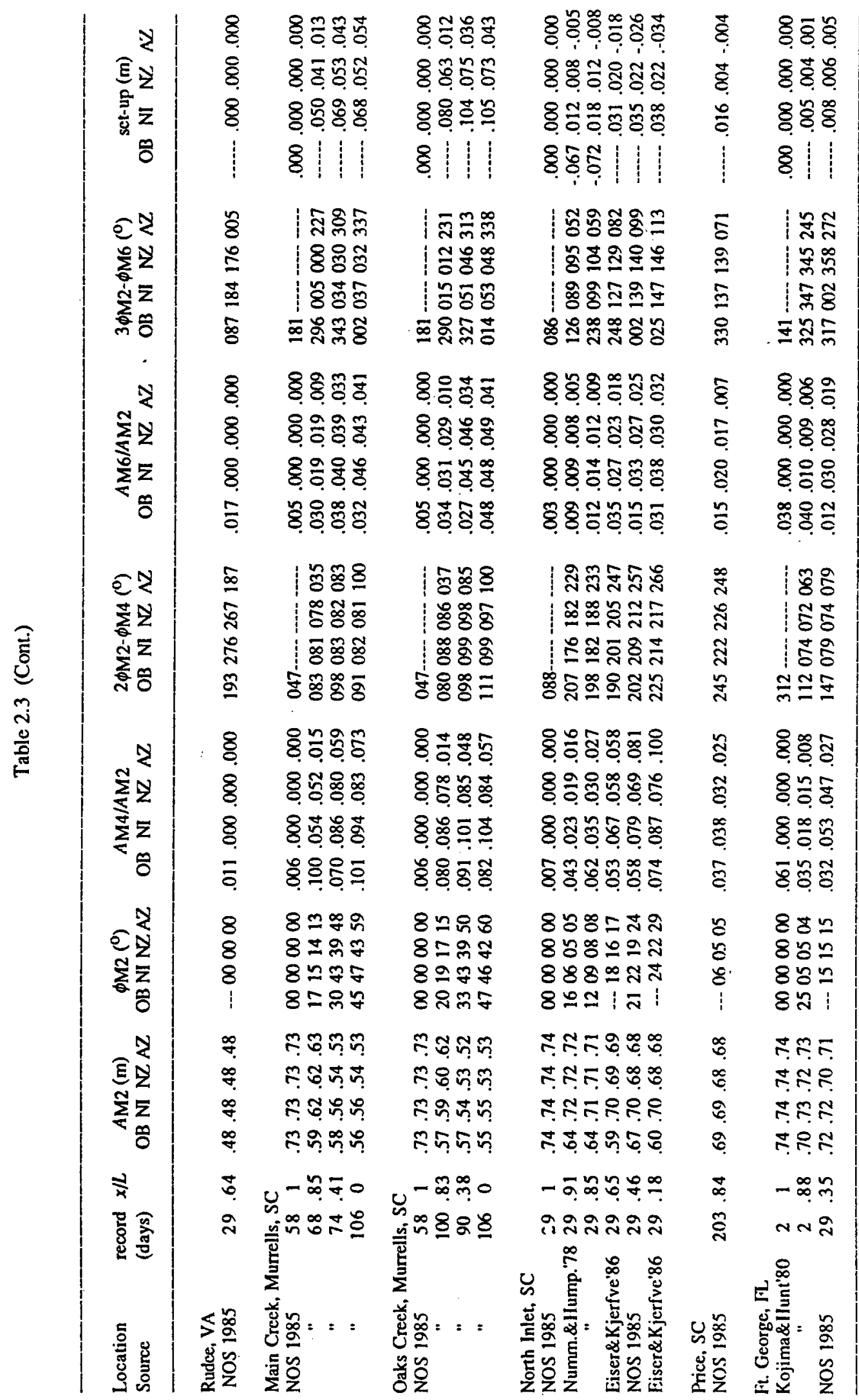


Figure captions for Chapter 2

Figure 2.1. Diagram of an idealized tidal embayment cross-section: $\zeta(x, t)$ is surface elevation relative to mean sea level (MSL) at the forced end of the embayment; $b_{c}$ and $h$ are the surface width and cross-sectionally averaged depth of the channel; $b$ is the total width of the embayment cross-section, including tidal flats which act in a storage capacity only; $b_{0}$ is the time-averaged width of the embayment cross-section (at an elevation not necessarily coinciding with MSL). Elevations $h_{1}$ and $h_{2}$ are used in specifying the geometry of the intertidal storage area. Vertical exaggeration is on the order of $100: 1$.

Figure 2.2 Time series of (2.15), the analytic solution to the linearized zero-inertia equation, during two tidal cycles calculated at $x / L=1,0.8,0.6,0.4,0.2$, and $0(x / L=0$ landward): (a) $\left\|k_{0}\right\| L=1 / 2$, (b) $\left\|k_{0}\right\| L=1$, (c) $\left\|k_{0}\right\| L=2$, and (d) $\left\|k_{0}\right\| L=4$.

Figure 2.3. Approximations of $\left.\operatorname{lcos}(\omega t+\phi+\pi / 4)\right|^{-1 / 2}$ : (a) exact is solid line; $(\pi / 2)^{1 / 2}(1-(1 / 3) \cos 2(\omega t+\phi+\pi / 4))$ is dashed line; (b) exact is solid line; $(\pi / 2)^{1 / 2}(1+$ $\delta \cos 2(\omega t+\phi+\pi / 4))$ is dashed line.

Figure 2.4. Time series of (2.40)-(2.41), (2.44), the approximate analytic solution of the zero-inertia equation with a time-varying diffusion coefficient, with $\left\|k_{0}\right\| L=$ 1 during two tidal cycles calculated at $x / L=1,0.8,0.6,0.4,0.2$, and $0(x / L=0$ landward): (a) $\gamma=0.5$, and (b) $\gamma=-0.5$.

Figure 2.5. Time series of (2.40)-(2.41), (2.44), the approximate analytic solution of the zero-inertia equation with a time-varying diffusion coefficient, with $\gamma=0.5$ during two tidal cycles calculated at $x / L=1,0.8,0.6,0.4,0.2$, and $0(x / L=0$ landward): (a) $\left\|k_{0}\right\| L=1 / 2$, (b) $\left\|k_{0}\right\| L=1$, (c) $\left\|k_{0}\right\| L=2$, and (d) $\left\|k_{0}\right\| L=4$.

Figure 2.6. Comparisons of numerical solutions to (2.1)-(2.2), the equations of motion including the inertia terms, with observations at 32 tide gauges in 12 tidal embayments (see Table 2.3): (a) $\mathrm{M}_{2}$ amplitude divided by $\mathrm{M}_{2}$ forcing amplitude, and (b) $\mathrm{M}_{2}$ phase (deg.) relative to forcing $\mathrm{M}_{2}$ phase. The solid line is unit slope, std is the standard deviation of the residuals from their mean, and bias is the mean residual.

Figure 2.7. Comparisons of numerical solutions to (2.1)-(2.2), the equations of motion including the acceleration terms, to numerical and approximate analytic solutions 
of the zero-inertia equation for 32 tide gauges in 12 tidal embayments (see Table 2.3): (a) $M_{2}$ amplitude divided by $M_{2}$ forcing amplitude, (b) $M_{2}$ phase (deg.) relative to forcing $M_{2}$ phase, (c) local $M_{4}$ to $M_{2}$ amplitude ratio, (d) local $M_{4}$ to $M_{2}$ relative phase (deg.), (e) local $\mathrm{M}_{6}$ to $\mathrm{M}_{2}$ amplitude ratio, and (f) local $\mathrm{M}_{6}$ to $\mathrm{M}_{2}$ relative phase (deg.). Numerical solution of (2.8), the fully nonlinear zero-inertia equation are circles; (2.40)-(2.41), (2.44), the approximate analytic solution with a time-varying diffusion coefficient are pluses. The solid line is unit slope, std is the standard deviation of the residuals from their mean, and bias is the mean residual.

Figure 2.8. Tidal surface elevation parameters as a function of distance for the tidal embayments at Chatham (pluses) and North Inlet (circles): (a) $\mathrm{M}_{2}$ amplitude divided by $M_{2}$ forcing amplitude, (b) $M_{2}$ phase (deg.) relative to forcing $M_{2}$ phase, (c) local $M_{4}$ to $\mathrm{M}_{2}$ amplitude ratio, and (d) local $\mathrm{M}_{4}$ to $\mathrm{M}_{2}$ relative phase (deg.). Field observations are dotted lines; numerical solutions to (2.1)-(2.2), which include the inertia terms, are solid lines; numerical solutions of (2.8), the fully nonlinear zero-inertia equation, are dashed lines; (2.40)-(2.41), (2.44), the approximate analytic solution with a time-varying diffusion coefficient, are dash-dot lines.

Figure 2.9. Comparisons of observations at 32 tide gauges in 12 tidal embayments (see Table 2.3) to (2.40)-(2.41), (2.44), the approximate analytic solution of the zero-inertia equation with a time-varying diffusion coefficient: (a) $M_{2}$ amplitude divided by $\mathrm{M}_{2}$ forcing amplitude, (b) $\mathrm{M}_{2}$ phase (deg.) relative to forcing $\mathrm{M}_{2}$ phase, (c) local $M_{4}$ to $M_{2}$ amplitude ratio, and (d) local $M_{4}$ to $M_{2}$ relative phase (deg.). The solid line is unit slope, std is the standard deviation of the residuals from their mean, and bias is the mean residual. 


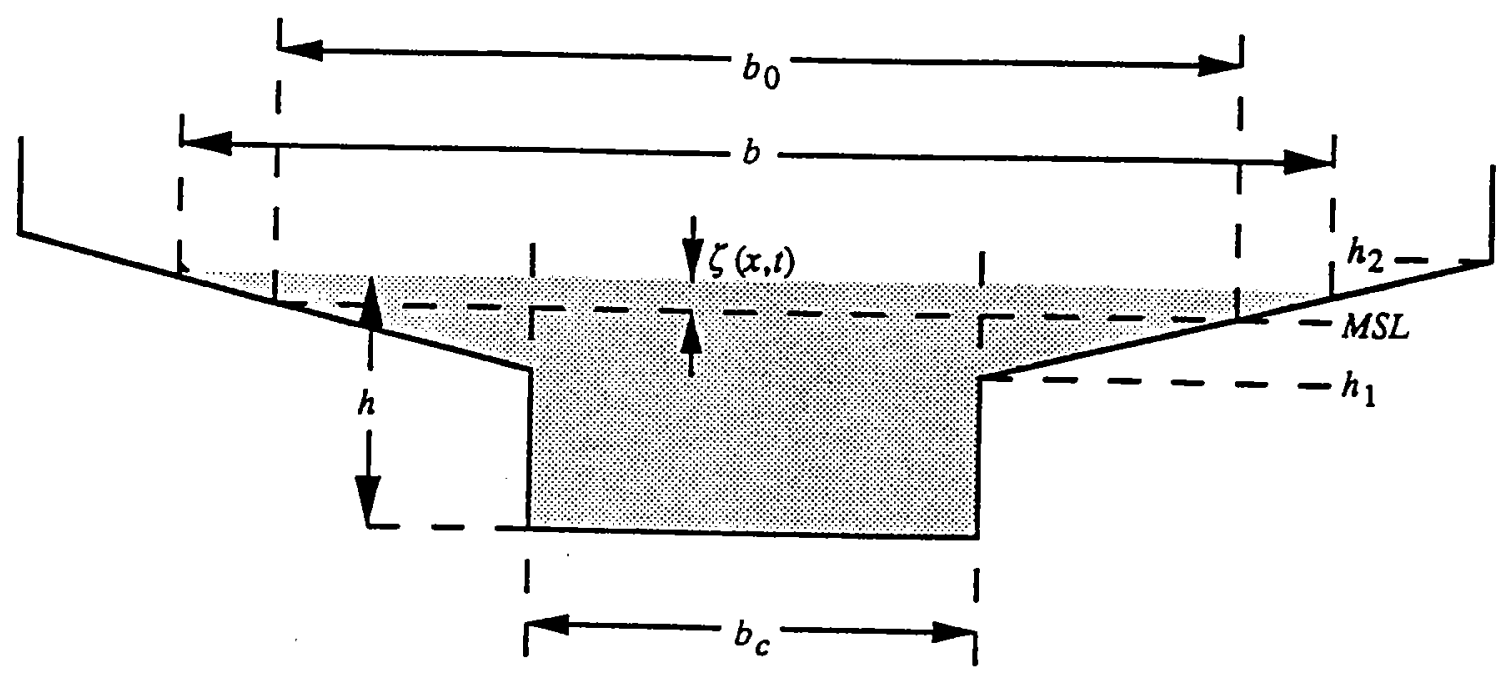

Figure 2.1 

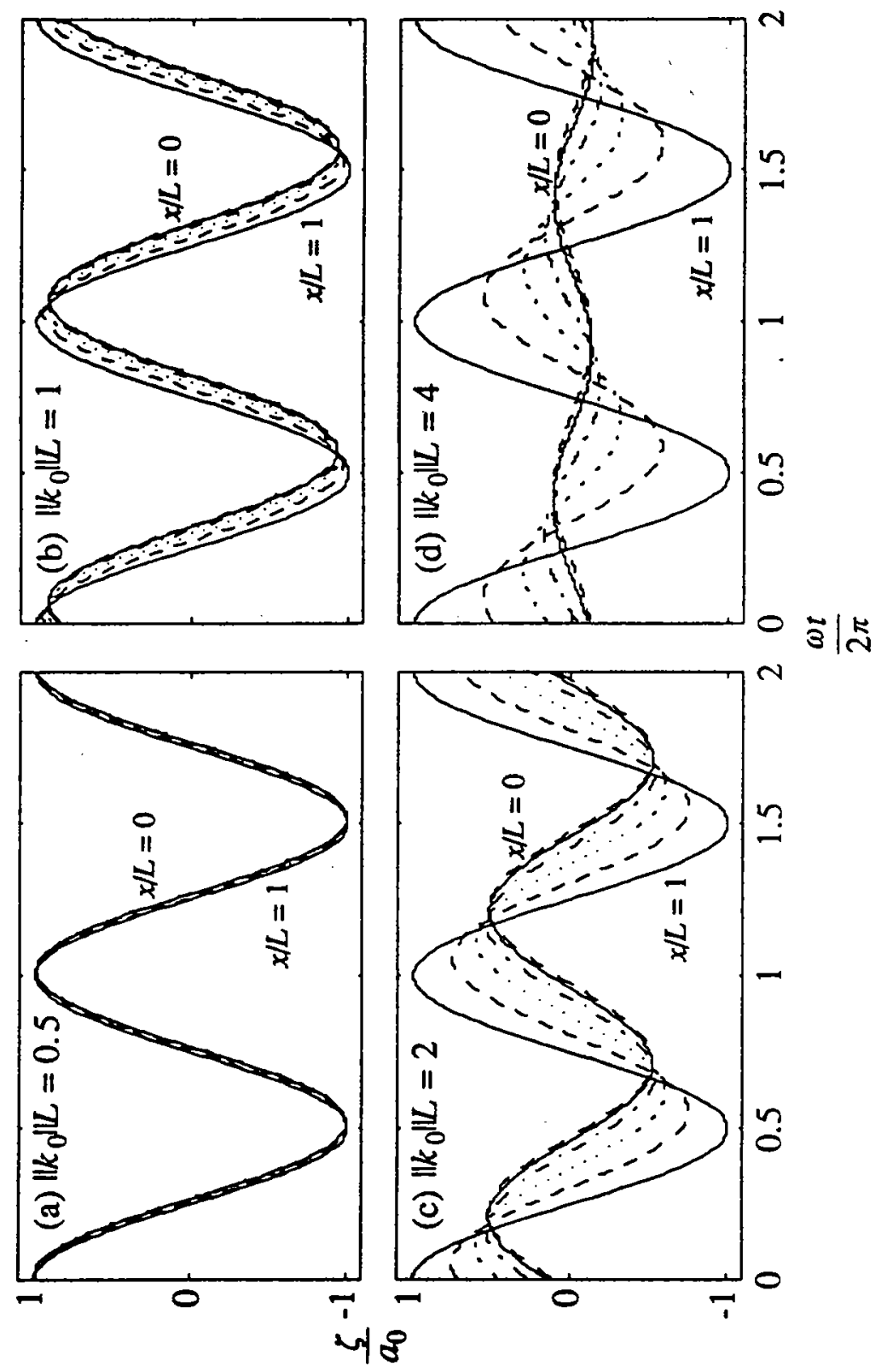

Figure 2.2 


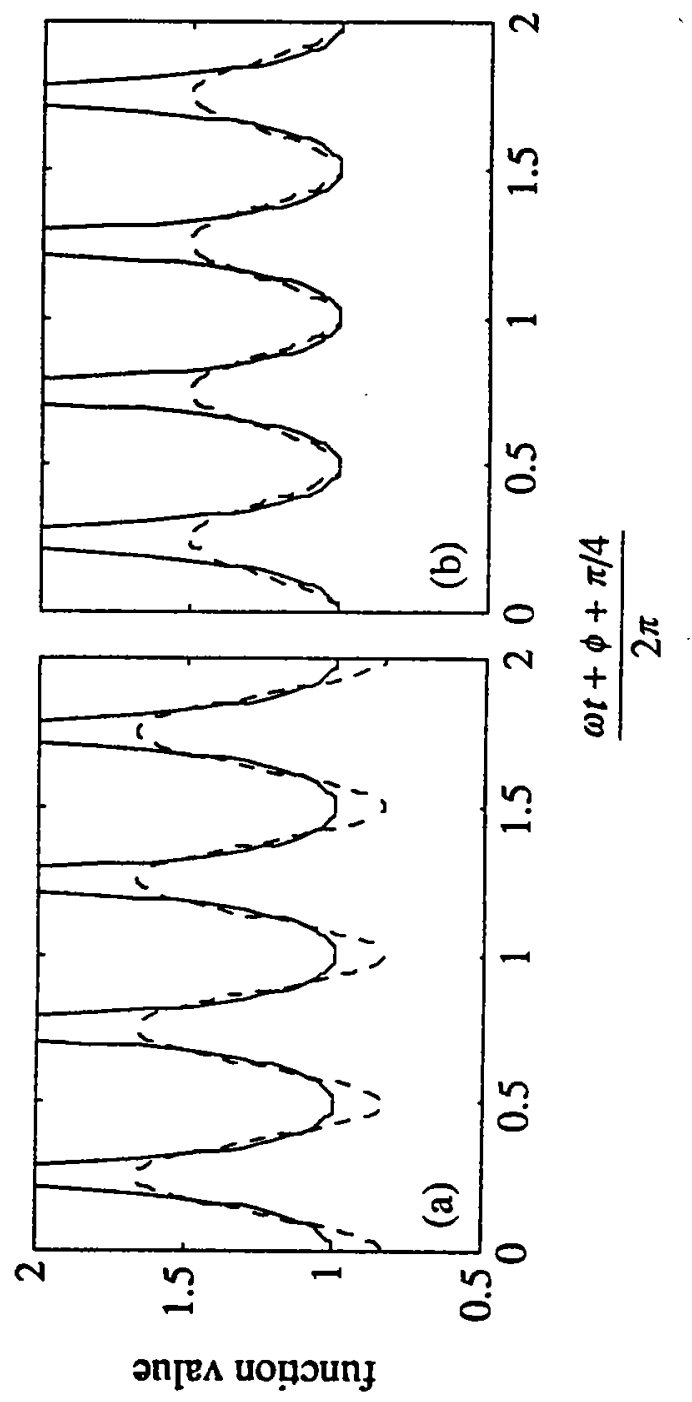

Figure 2.3 


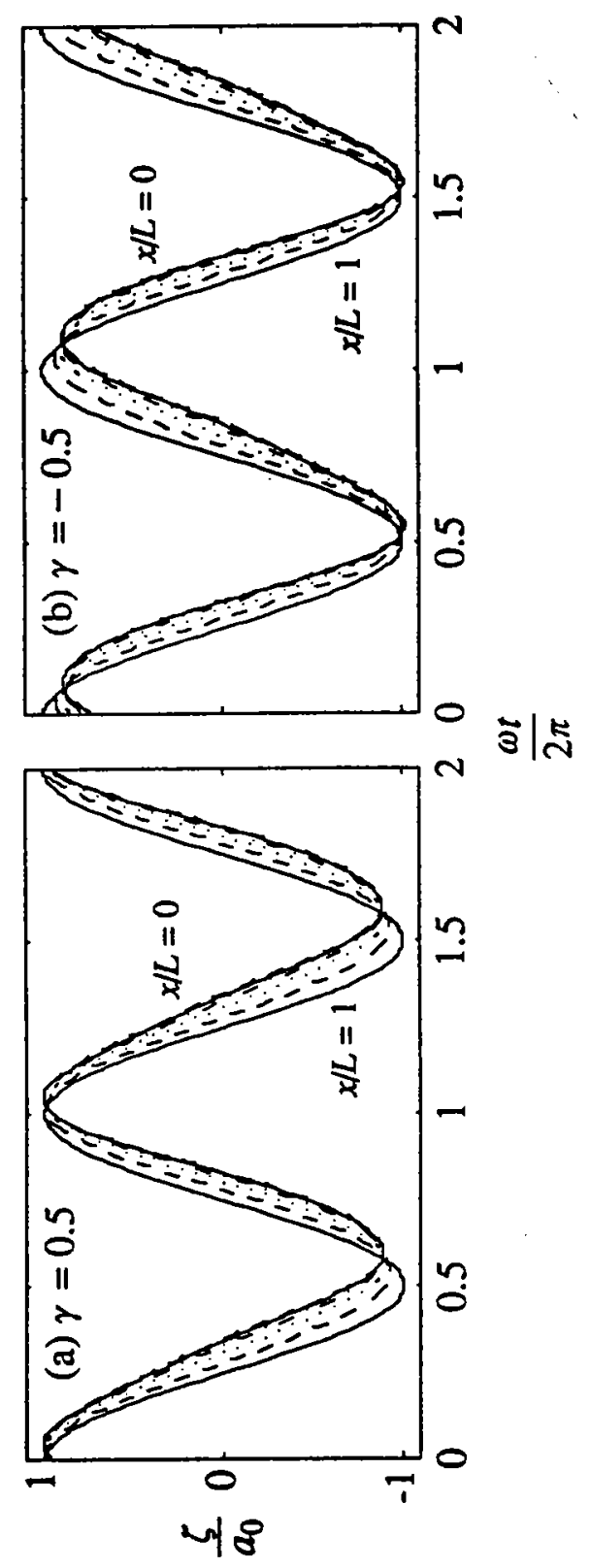

Figure 2.4 

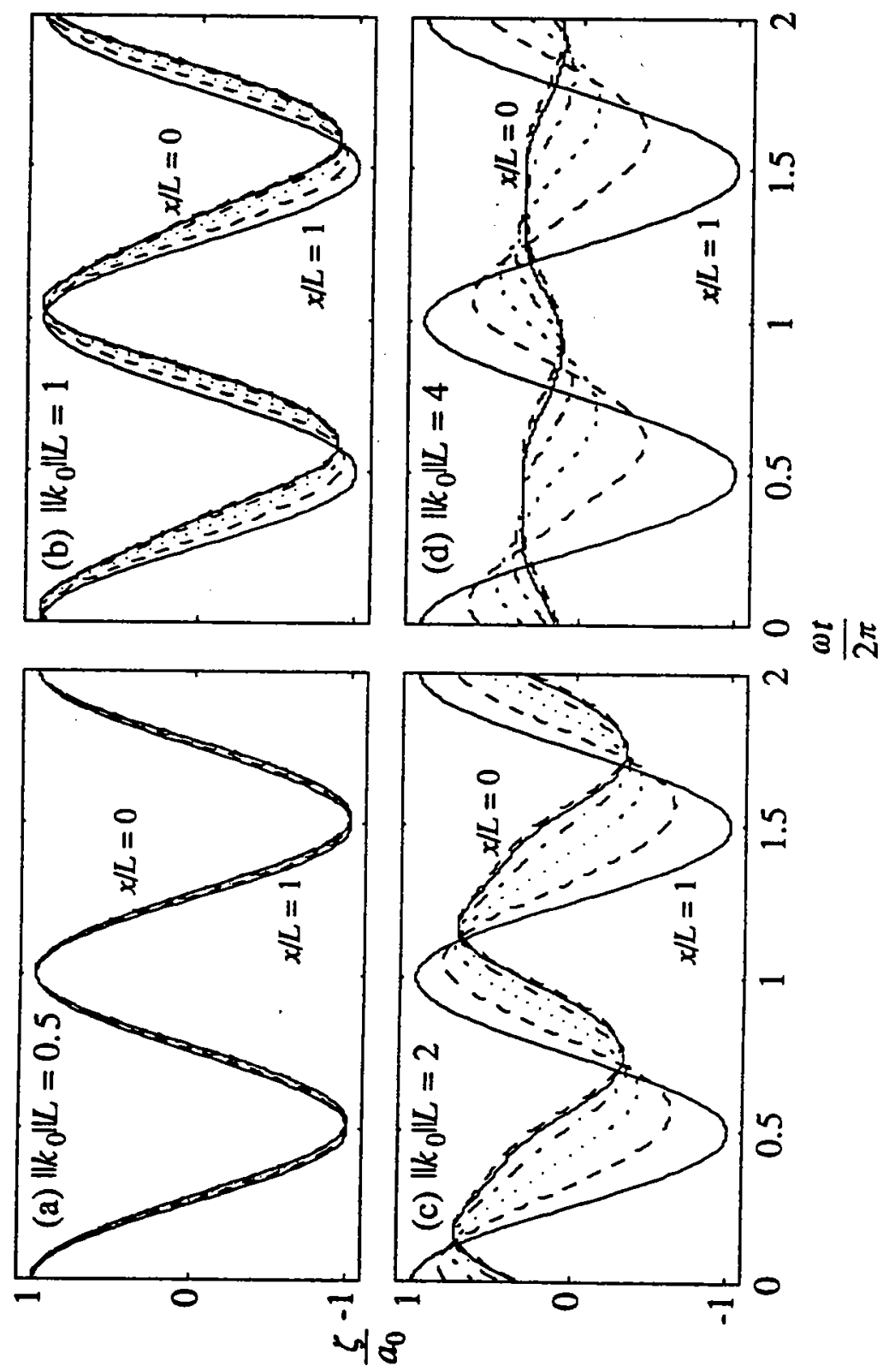

Figure 2.5 

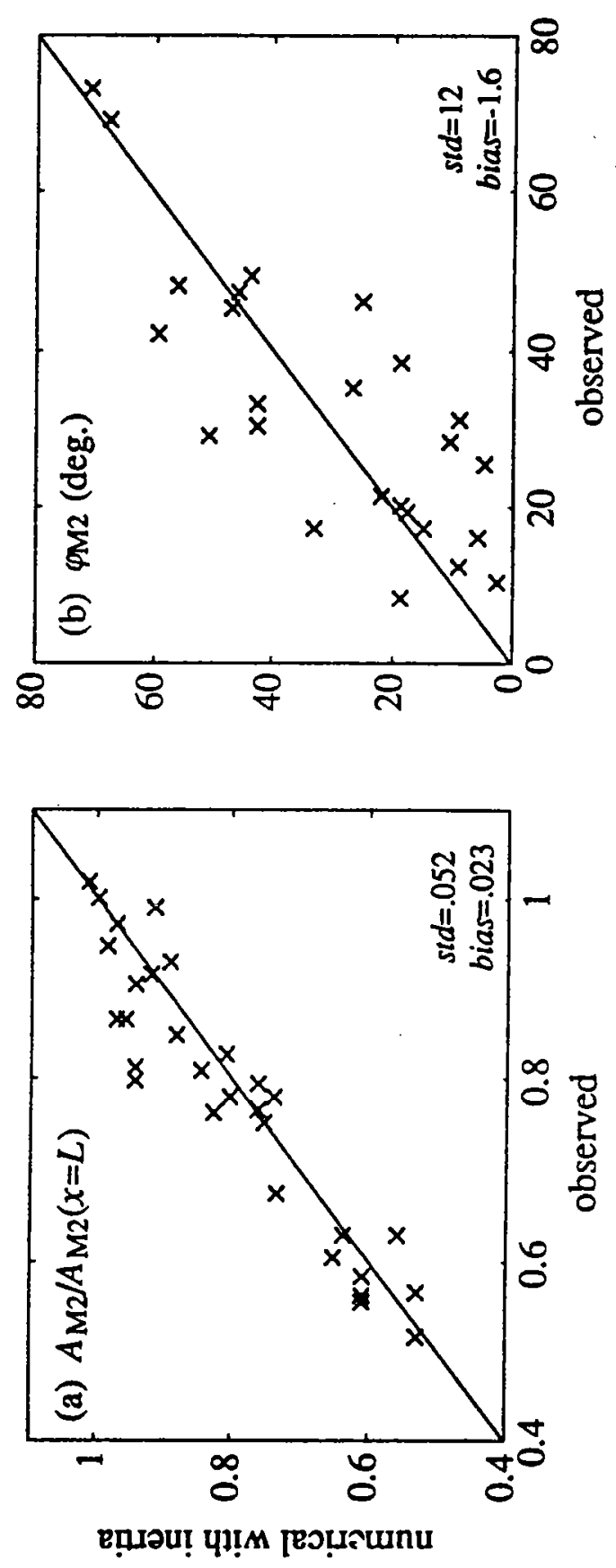

Figure 2.6 

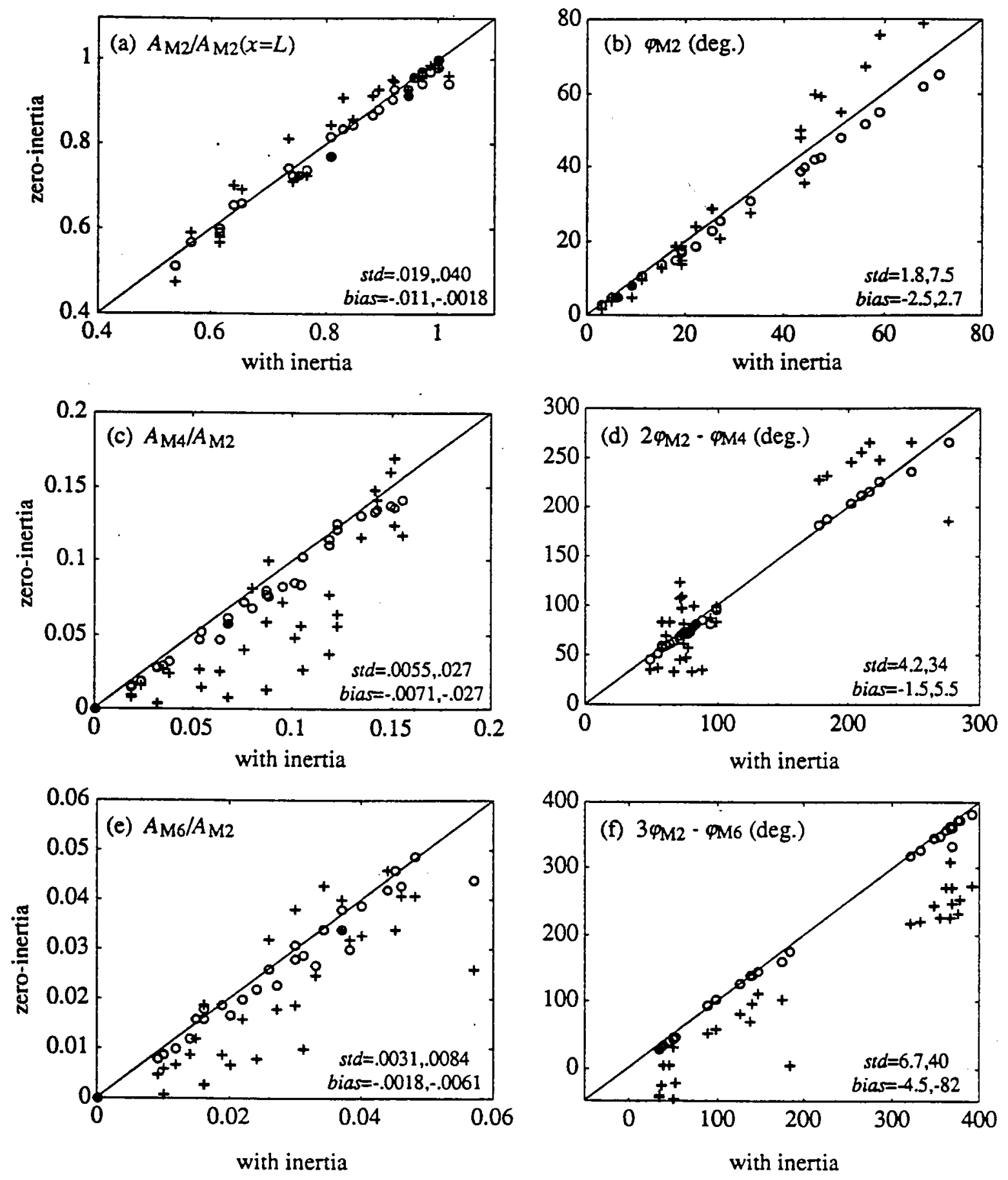

Figure 2.7 

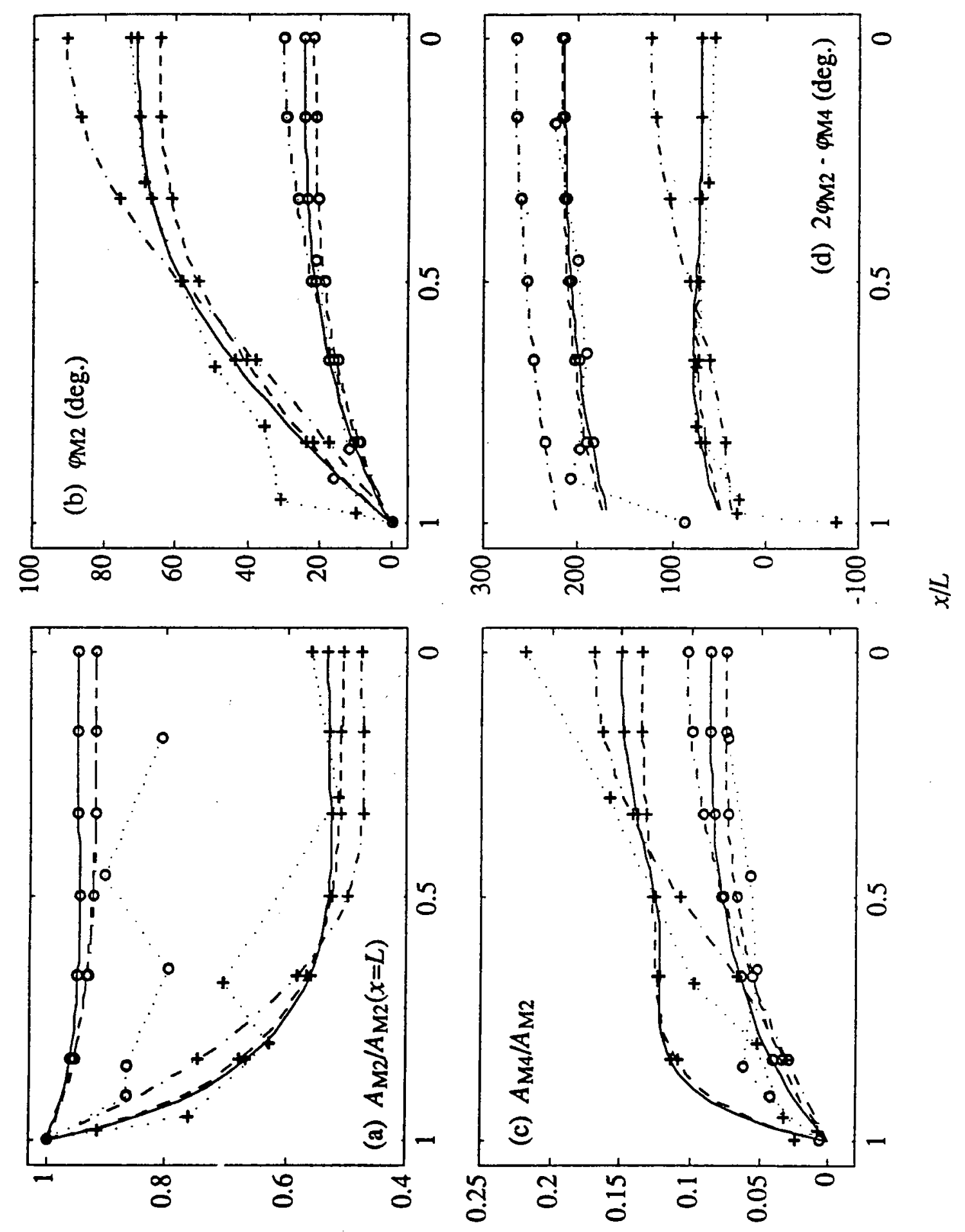

Figure 2.8 

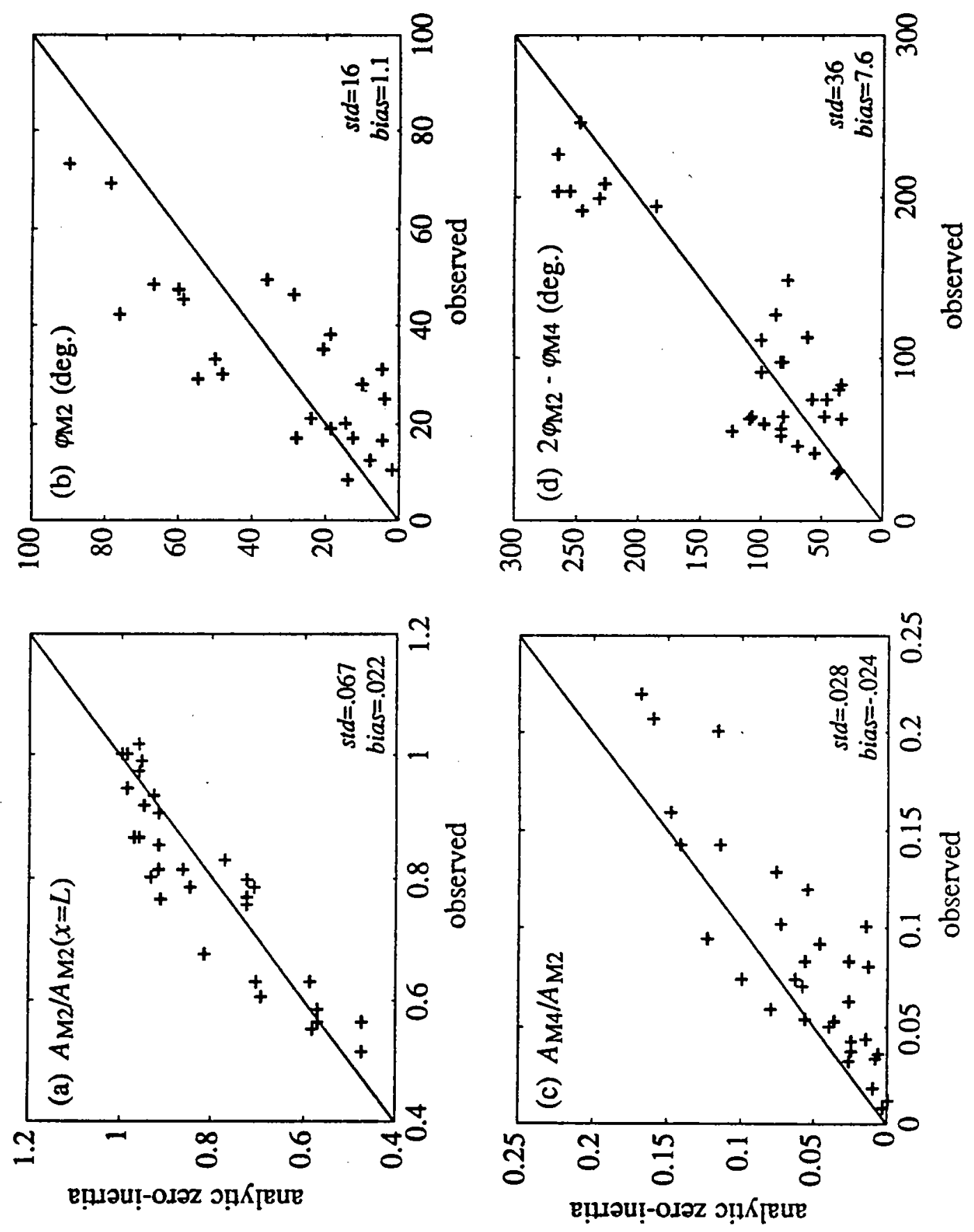

Figure 2.9 


\section{Chapter 3:}

Stability Shear Stress and Equilibrium Geometry of Tidal Channels and Tidal Embayments 


\section{Abstract to Chapter 3}

This study relates channelized tidal embayment morphology to flow properties via a physical mechanism, namely the stability shear stress $\left(\tau_{s}\right)$ at peak discharge just necessary to maintain zero gradient in net along-channel sediment transport. A survey of the literature provides estimates of peak discharge at spring tide $(Q)$, channel cross-sectional area $(A)$ and hydraulic radius $\left(h_{R}\right)$ at 146 sections in 18 separate tidal systems for use in applying this concept.

A theoretical lower bound on $\tau_{s}$ (and an upper bound on cross-sectional geometry) is provided by the critical shear stress just capable of initiating sediment motion. Application of critical shear stress theory predicts along-channel geometry will follow the relation $A h_{R}{ }^{1 / 6} \sim Q$. Along-channel regressions of the form $A h_{R}^{1 / 6} \sim Q^{\beta}$ give a mean observed value for $\beta$ of $1.01 \pm 0.04$, which is indistinguishable from one and, therefore, consistent with critical shear stress theory. However, the equation $A h_{R}^{1 / 6} \sim Q$ is consistent with any uniform value of along-channel $\tau_{s}$, and observations indicate $\tau_{s}$ in individual systems can vary widely above the value predicted by initiation of sediment motion.

Observed $\tau_{s}$ is found to vary among all systems according to the relation $\tau_{s} \approx$ $1.7 R_{s p}{ }^{0.8} \tau_{c}$, where $R_{s p}$ (in meters) is spring tidal range, and $\tau_{c}$ is the total shear stress at the initiation of sediment motion. $\tau_{s}$ may vary with $R_{s p}$ because of an associated increase in sediment supply or because of a correlation between $R_{s p}$ and characteristic patterns of discharge asymmetry. Observed deviations from uniform $\tau_{s}$ along individual channels are associated with along-channel variation in the direction of maximum discharge. It is hypothesized that a convergence in the direction of maximum discharge may cause deposition, a reduction in $A$, and a local increase in $U=Q / A$ until a locally increased $\tau_{s}$ is reached which prevents further deposition. Geometries and discharge asymmetries along several channels are observed to be consistent with this pattern.

Finally, an assumption of uniform along-channel $\tau_{s}$ is used to derive equilibrium along-channel geometries for entire embayments as a function of storage in flats and marsh. Theoretical predictions are consistent with published observations from Wrecked Recorder Creek, Virginia, and the Ord River in Western Australia. The moiphologic tendency towards uniform $\tau_{s}$ has important ramifications concerning classical views of mass and momentum balances, mechanisms for net sediment transport, and mathematical formulations of along-channel mixing coefficients. 
List of symbols in Chapter 3

A channel cross-sectional area

$b$ time-averaged embayment width

$b_{0}, b_{L} \quad b$ at $x=0$ or $x=L$

$b_{S I I W} b$ at spring high water

$b_{S L W} \quad b$ at spring low water

d grain diameter

$g \quad$ acceleration of gravity

$G$ specific weight of sediment in fluid

$h$ cross-sectionally averaged depth

$h_{R} \quad$ hydraulic radius

$K$ eddy diffusivity

$L \quad$ total length of tidal channel

$L_{e} \quad$ tidal excursion length

$n \quad$ Manning's friction coefficient

$q \quad$ discharge

$Q \quad$ peak spring $q$

$Q_{e b b} \quad$ peak spring $q$ during ebb

$Q_{\text {flood }}$ peak spring $q$ during flood

$R_{\text {obs }}$ observed tidal range

$R_{s p} \quad$ spring tidal range

s.e. standard error

$t$ time

. $T$ semi-diurnal tidal period

$u \quad$ velocity

$\bar{u} \quad$ depth-averaged $u$

$U \quad$ cross-sectionally averaged $u$

$U_{c} \quad U$ at the initiation of sediment motion

$w \quad$ channel width

$x \quad$ along-channel co-ordinate

$z \quad$ height above bottom

$z_{0} \quad$ length scale related to bottom roughness

$Z \quad$ amplitude of $\zeta$

$Z_{0}, Z_{L} \quad Z$ at $x=0$ or $x=L$. 
List of symbols in Chapter 3 (continued)

$\alpha \quad$ regression coefficient for $A \sim Q^{\alpha}$

$\beta \quad$ regression coefficient for $A h_{R}{ }^{1 / 6} \sim Q^{\beta}$

$\Delta f \quad$ error in generic variable or function $f$

$\zeta \quad$ tidal elevation

$\kappa \quad$ von Karman's constant

$\lambda_{b}, \lambda_{z}$ constant describing exponential variation of $b$ or $Z$

$\rho \quad$ fluid density

$\sigma_{b}, \sigma_{z}$ constant describing power-law variation of $b$ or $Z$

$\tau \quad$ total bottom shear stress

$\tau^{\prime} \quad$ grain shear stress

$\tau^{\prime \prime} \quad$ form drag

$\tau_{c} \quad \tau$ at the initiation of sediment motion

$\tau_{c}^{\prime} \quad \tau^{\prime}$ at the initiation of sediment motion

$\tau_{e} \quad$ erosion shear stress for cohesive mud

$\tau_{\theta}^{\prime} \quad \tau_{c}^{\prime}$ on channel bank of slope $\theta$

$\tau_{s} \quad$ stability shear stress

$\psi_{c} \quad$ critical Shields parameter

$\omega$ tidal frequency

$\Omega \quad$ spring tidal prism 


\subsection{Introduction}

Feedback between tidal channel morphology and tidal flow properties has long been recognized by coastal engineers and geologists (see text by Bruun 1976, for example). Deepening of tidal channels by dredging may reduce peak tidal velocity to a level below that necessary for sediment transport, causing accelerated deposition and an eventual return to an equilibrium channel depth. In contrast, a reduction of tidal prism by infilling or diking of marsh or lagoons may reduce velocities at a tidal inlet and cause deposition, leading to a smaller equilibrium cross-sectional area. Changes in channel morphology can occur on rapid time scales, with inlet cross-sectional area fluctuating by $10-15 \%$ over only a few days in response to variations in discharge due to storms or the spring-neap cycle (Byrne et al. 1975). Geologists have also noted the long-term impact on channel morphology of changes in tidal prism brought about by submergence or emergence of the tidal watershed (Gardner and Bohn 1980).

Qualitative effects such as these have motivated many'investigators to relate empirically the cross-sectional area of tidal channels and/or inlets to flow parameters, most commonly to spring tidal prism (e.g., O'Brien 1931) or to peak spring discharge (e.g., Chantler 1974). For short inlet channels connecting bays or lagoons to the ocean, these empirical controls have been synthesized with hydrodynamic relations, resulting in stability curves for inlet cross-sectional area (e.g., Escoffier 1977; van de Kreeke 1990). Relatively less attention has been paid to the morphodynamics of longer tidal channels typically associated with the interiors of tidal marshes and with the lower reaches of tidal rivers. Yet morphodynamic relations for these channelized tidal embayments are arguably simpler and more closely related to fundamental physics. Tidal channels well within embayments are isolated from the complicating effects of direct wave attack and littoral drift and are generally subjected to less severe spatial gradients in tidal amplitude and phase than are channels within tidal inlets.

The purpose of this study is to relate channelized embayment morphology to flow properties via a physically-based mechanism, namely the "stability" shear stress $\left(\tau_{s}\right)$ just uecessary to maintain a zero gradient in net along-channel sediment transport. It is assumed that if the peak shear stress during spring tides is locally greater than $\tau_{s}$, then net erosion will occur, whereas if it is less than $\tau_{s}$ there will be net deposition. At first the problem is simplified by assuming stability is reached when grain shear stress is everywhere equal to the critical level necessary for initiation of sediment motion. Resulting 
theoretical relations between cross-sectional area and peak spring discharge are compared to observations from the literature taken at 146 cross-sections in 18 separate tidal systems. Next, likely causes of observed deviations from this simplest application of $\tau_{s}$ theory are discussed. Among tidal channels, increases in $\tau_{s}$ above that predicted by initiation of sediment motion are found to be correlated with increased tidal range. Along-channel variations in $\tau_{s}$ are hypothesized to result from systematic along-channel patterns of velocity asymmetry. Finally, the above morphologic controls are combined with continuity of fluid flow to predict equilibrium along-channel geometries for entire embayments. Geometric predictions for entire embayments are compared to observations from two systems which have previously been the subject of morphodynamic investigations, namely Wrecked Recorder Creek, Virginia (Myrick and Leopold 1963), the Ord River in Western Australia (Wright et al. 1973).

The present application of $\tau_{s}$ theory to tidal channel morphology involves several simplifying assumptions. Primary among them is the assertion that bottom shear stress can be related to the cross-sectionally averaged amplitude of the current. This requires densitydriven currents to be at most second-order, but does not require fresh water discharge to be zero or even negligible. It is also assumed that contributions to bottom stress by winddriven currents and waves are negligible. Hence this analysis does not address the equilibrium morphology of inlet channels exposed to significant wave activity and/or littoral drift. Another limitation of the present argument is its emphasis on non-cohesive sediment. The simplest form of $\tau_{s}$ theory relies in part on the Shields entrainment function for the initiation of grain motion (e.g., Yalin 1977) and other relations based exclusively on noncohesive material. However the Shields criterion can be replaced with another critical erosion parameter based on studies of cohesive sediment (e.g., Dyer 1986), and the fundamental results still remain.

\subsubsection{Previous observations of equilibrium tidal channels}

A survey of the literature (Table 3.1) reveals about a dozen observational studies which quantitatively relate cross-sectional morphology to flow along tidal channels or through tidal inlets sheltered from offshore wave activity. Many of the authors in Table 3.1 noted cross-sectional area $(A)$ to be nearly proportional to either spring tidal prism $(\Omega)$ or peak discharge $(Q)$ through relations of the form

$$
A \sim \Omega^{\alpha} \text { or } A \sim Q^{\alpha} \text {, }
$$


where $\alpha \approx 1$, and $A$ corresponds to the time of peak $Q$. If discharge is assumed to be sinusoidal, then

$$
Q=\frac{\pi}{T} \Omega
$$

where $T$ is the tidal period, and the two relations in (3.1.1) become interchangeable. However, time-series of discharge in tidal channels are often strongly asymmetric, especially in channels having large amplitude-to-depth ratios and in the upper reaches of well-mixed channels having finite fresh-water input. For the purposes of this study, direct observations of $Q$ are preferable to estimates calculated via (3.1.2).

Figure 3.1 contains values for $Q$ and $A$ determined from information published in the sources listed in Table 3.1. The discharges in Figure 3.1 are (in order of preference) either (i) taken directly from published values of $Q$, (ii) calculated from published values of cross-sectionally averaged peak velocity, $U$, such that

$$
Q=A U
$$

or (iii) calculated from published values of $\Omega$ via (3.1.2). Here $Q$ is (ideally) defined as the magnitude of maximum discharge under spring tide conditions. Where $Q$ corresponds to a known stage of the fortnightly cycle other than spring, then $Q$ is scaled by the ratio of the mean spring tidal range $\left(R_{s p}\right)$ to the range at the time of the discharge measurement $\left(R_{o b s}\right)$. $R_{s p}$ is taken either from the sources in Table 3.1, from N.O.A.A. tide tables, or from information on U.S.D.M.A. bathymetric charts. Where tides are of the mixed type (i.e., San Francisco and Oregon in Table 3.1), $R_{s p}$ is defined as the difference between mean higher high water and mean lower low water. Errors in $Q$ are estimated to be on the order of $20 \%$.

Wherever possible, $A$ is the area of the wetted cross-section at the time of $Q$. More often, however, the precise area at the time of $Q$ is unavailable, and the cross-sectional area below mean tide level is used instead. Nonetheless, errors in the measurement of $A$ are likely to be smaller than errors in the measurement of $Q$. Here $A$ is estimated to be accurate to within 5\%. Table 3.1 includes only locations for which values for cross-sectionally averaged depth $(h)$ are also available. All but two of the sources in Table 3.1 include $h$ directly (or at least width, $w$, so that $h=A / w$ can be calculated). Widths for the Western Scheldt sections were taken from Gerritsen et al (1991), and widths for the sheltered Australian inlets were obtained from U.S.D.M.A. charts H.O. 3451 and N.O. 74183. 
Finally, the seaward-most cross-section of several of the channels (Alsen, Siletz, Yaquina, Western Scheldt, and Ems) were purposely excluded from the analysis because of clear morphodynamic alteration by ocean waves and associated littoral drift.

\subsubsection{Previous explanations for proportionality of area and discharge}

Published explanations for the near proportionality of $A$ and $Q$ in tidal channels include maximum entropy (Myrick and Leopold 1963; Wright et al. 1973), uniform critical velocity (Chantler 1974; Riedel and Gourlay 1981; Byrne et al. 1981), a form of $\tau_{s}$ theory based on plane bed flow (de Jong and Gerritsen 1985), or merely agreement with previous empirical relationships (Goodwin et al. 1970; O'Connor et al. 1991). From an analogy to thermodynamics, the maximum entropy hypothesis states that tidal channel geometry adjusts toward a uniform distribution of energy dissipation and a minimum rate of work in the system as a whole. Uniform energy dissipation can be re-expressed as a uniform distribution of shear stress, a concept which is consistent with the present study. Minimum work, however, is not connected directly to the equations governing tidal flow and sediment motion. Thus the maximum entropy hypothesis will not be pursued further in this paper.

In its simplest form, the critical velocity $\left(U_{c}\right)$ hypothesis states that $A$ adjusts until a characteristic cross-sectionally averaged $U=U_{c}$ causes a bottom shear stress just capable of dislodging material from the channel bed and banks (Chantler 1974). If $U>U_{c}$, net erosion will increase the section's area, and $U$ will decrease. Conversely, if $U<U_{c}$, net deposition will decrease the section's area, and $U$ will increase. This concept is more properly termed critical shear stress theory since bottom shear stress, rather than $U$, is dynamically linked to initiation of sediment motion. In open channel flow, boundary shear stress is strongly dependent on $U$ and weakly dependent on depth (Henderson 1966). Thus $U_{c}$ should vary weakly as a function of depth among tidal channels as well as along the length of individual channels. In fact, the mean value of $\alpha$ in Table 3.1 is a bit less than one, suggesting a slight decrease in $U$ typically occurs as $Q$ and depth decrease together along the length of individual channels.

Krishnamurthy (1977) applied a criterion related to critical shear stress theory to his study of tidal inlet morphology in the absence of littoral drift. Krishnamurthy suggested that for morphologic equilibrium, the time-averaged magnitude of bottom shear stress in the inlet should be no greater than the critical value required for sediment motion In the study of bed load transport by tidal currents, however, it is generally agreed that the peak value of 
bottom shear stress is a more relevant parameter than its time-averaged magnitude (e.g., Bruun 1967; Pingree and Griffiths 1979).

The form of $\tau_{s}$ theory applied by de Jong and Gerrittsen (1985) was originally developed for tidal inlets subject to significant littoral drift (Bruun and Gerritsen 1960; Bruun 1967). Bruun (1967) observed $U$ to be $1 \mathrm{~m} / \mathrm{s} \pm 15 \%$ at seventeen sandy inlets of various sizes distributed across northern Europe and the east, west and Gulf coasts of the United States. According to Bruun, $A$ adjusts until the total bottom shear stress produced by $U$ flushes away the most possible sediment with the least possible frictional loss. In many inlets, $U \approx 1 \mathrm{~m} / \mathrm{s}$ is just sufficient to flatten dunes and produce a plane bed, thereby applying the maximum portion of available total shear stress directly to the bottom material. A similar mechanism may apply to tidal channels which are subjected to large inputs of sediment. However a stability theory based solely on plane bed flow is inadequate for a generalized study, for $U$ is well below $1 \mathrm{~m} / \mathrm{s}$ in many stable tidal channels.

\subsection{Stability shear stress given by critical shear stress}

In this study, shear stress $\left(\tau_{s}\right)$ is defined as the total bottom shear stress just necessary to maintain a zero along-channel gradient in net sediment transport. The lower bound on $\tau_{s}$ can be derived from the condition $\tau^{\prime}=\tau_{c}^{\prime}$, where $\tau^{\prime}$ is maximum grain shear stress and $\tau_{c}{ }_{c}$ is the critical grain shear stress necessary for initiation of sediment motion. This end member is the simplest form of $\tau_{s}$ theory and is also known as critical shear stress theory. In this section, previous applications of critical shear stress in unidirectional flow are briefly reviewed. Critical shear stress theory is then applied to the equilibrium geometry of tidal channels. Finally, theoretical predictions of cross-sectional geometry versus discharge are compared to observations from 146 cross-sections in 18 separate tidal systems.

\subsubsection{Insight from unidirectional flow}

Critical shear stress theory has long been applied to the design of stable canals under conditions of unidirectional flow (e.g., I ane 1955; Henderson 1966). Where zero scour of the canal beds and banks is desired, the limiting design condition is that $\tau^{\prime}$ is no greater than $\tau_{c}^{\prime}$ at any point of the channel boundary. However, more recent investigations of self-formed sand channels (Parker 1978; Diplas 1990) indicate a dynamic equilibrium is possible only if $\tau^{\prime}$ is slightly greater than $\tau_{c}^{\prime}$, therefore allowing the presence of a small but finite bedload. This is inconsistent with a critical shear stress theory based solely on the 
initiation of sediment motion, in that $\tau^{\prime}>\tau_{c}^{\prime}$ should cause erosion of the channel banks and instability. To allow $\tau^{\prime}>\tau_{c}^{\prime}$ while maintaining equilibrium, a lateral diffusion mechanism supplements the critical shear stress model (Parker 1978; Diplas 1990). In the presence of finite bed load transport, lateral diffusion of momentum due to turbulence continually moves grains from the channel axis toward the channel banks, counteracting the bank erosion caused by $\tau^{\prime}>\tau_{c}^{\prime}$. Laboratory experiments with self-formed sand channels confirm that at equilibrium, $\tau^{\prime}$ along the channel axis is up to $15 \%$ greater than $\tau_{c}^{\prime}$ (Diplas 1990).

In this investigation it is assumed that a single representative value for critical shear stress may be reasonably applied to the entire perimeter of the tidal channel. Actually, $\tau_{c}$ will be somewhat lower on steep portions of the channel bank due to the disturbing force of the grain's weight resolved down the bank slope. A balance of forces on a non-cohesive grain shows that the shear stress for initiation of motion on the bank $\left(\tau_{\theta}^{\prime}\right)$ relative to that on a flat bed $\left(\tau_{c}^{\prime}\right)$ is given by

$$
\frac{\tau_{\theta}^{\prime}}{\tau_{c}^{\prime}}=\cos \theta \sqrt{1-\frac{\tan ^{2} \theta}{\tan ^{2} \phi}},
$$

where $\theta$ is the bank slope angle, and $\phi \approx 30^{\circ}$ is the angle of repose of the sediment (Henderson 1966). The derivation of (3.2.1) requires the dynamic lift force on the grain to be small with respect to the drag force, an assumption consistent with the results of laboratory experiments (Ikeda 1982). The mean width-to-depth ratio of cross-sections used in Figure 1 is 150 , so the (larger than bedform scale) slope of the channel bed may be assumed to be negligibly small over the vast majority of any typical section.

\subsubsection{Application to tidal channels}

Critical shear stress theory is used to constrain the form of equilibrium tidal channel cross-sections as a function of discharge via the following steps: (i) relating $U=Q / A$ to total bottom shear stress, $\tau$, (ii) relating $\tau$ to grain shear stress, $\tau$, and (iii) requiring $\tau^{\prime}=$ $\tau_{c}^{\prime}$ at equilibrium.

If flow is assumed to be steady, two-dimensional, uniform and fully rough turbulent, then the familiar log-layer solution can be derived via dimensional analysis (e.g., Yalin 1977):

$$
u=\frac{1}{\kappa} \sqrt{\frac{\tau}{\rho}} \ln \left(\frac{z}{z_{0}}\right)
$$


where $\kappa=0.4$ is von Karman's constant, $\rho$ is fluid density, $z$ is height above the bottom, and $z_{0}$ is a length scale related to the bottom roughness. Integrating (3.2.2) over the depth of the water column, $h$, gives

$$
\bar{u}=\frac{1}{\kappa} \sqrt{\frac{\tau}{\rho}} \ln \left(\frac{h}{z_{0} \mathrm{e}}\right)
$$

where $\bar{u}$ is depth-averaged velocity. If $\tau$ is constrained to equal some critical value at equilibrium, (3.2.3) indicates that depth-averaged velocity should decrease weakly with decreased $h$.

An alternative equation for well-behaved flow in open channels is given by the more empirically based Manning-Strickler formula (e.g., Henderson 1966):

$$
U=\frac{Q}{A}=\frac{1}{n} \sqrt{\frac{\tau}{\rho g}} h_{R}^{1 / 6},
$$

where $g$ is the acceleration of gravity, $n$ is Manning's friction coefficient (with metric units of $\mathrm{m}^{-1 / 3} \mathrm{~s}$ ), and $h_{R}$ is the hydraulic radius of the channel. Whereas (3.2.3) assumes uniform two-dimensional flow, (3.2.4) is based on observations of three-dimensional flow in natural rivers and large man-made channels. The form of (3.2.4) inherently incorporates the effects of cross-channel depth variations and channel bends. Furthermore, $\tau$ in (3.2.4) can be treated as a characteristic total bottom shear stress for the cross-section as a whole. Thus (3.2.4) will be used in this study to relate $U$ to $\tau$. According to Henderson (1966, Table 4-2), typical values of $n$ for natural rivers are 0.025 to $0.030 \mathrm{~m}^{-1 / 3} \mathrm{~s}$ for "clean and straight" channels and 0.033 to $0.040 \mathrm{~m}^{-1 / 3}$ s for those that are "winding, with pools and shoals". A reasonable value of $n$ for equilibrium tidal channels, then, should be about $0.03 \pm 0.005 \mathrm{~m}^{-1 / 3} \mathrm{~s}$.

Following the suggestion of Einstein (1950), total shear stress ( $\tau$ ) is typically related to grain shear stress $\left(\tau^{\prime}\right)$ by $\tau=\tau^{\prime}+\tau^{\prime \prime}$, where $\tau^{\prime \prime}$ is bedform drag. A survey of the literature reveals relatively few direct measurements of the ratio $\tau / \tau$ over naturally formed bedforms (Table 3.2). The few values that have been reported over sand range widely from less than 0.1 to nearly 0.7 . Some of the disagreement in Table 3.2 results from the precise location of measurement. Kapdasli and Dyer (1986) measured $\tau^{\prime} / \tau$ directly above the ripple crest, where $\tau^{\prime \prime}$ is largest. The other measurements in Table 3.2 are spatially averaged. In their review paper, Engelund and Fredsoe (1982) suggest that when $\tau$ ' is only slightly greater than $\tau_{c}^{\prime}, \tau^{\prime} / \tau \approx 0.5$ in the presence of ripples and $\tau^{\prime} / \tau \approx 0.3$ in the 
presence of dunes. Since spatially averaged values of $\tau^{\prime} / \tau$ are needed here, a reasonable estimate for tidal channels in non-cohesive sand should be $0.4 \pm 0.2$.

For uniform, non-cohesive sediment under rough turbulent flow, dimensional analysis indicates the following relation should hold at the initiation of sediment motion (e.g., Yalin 1977):

$$
\psi_{c}=\frac{\tau_{c}^{\prime}}{\rho g G d}
$$

where $G$ is the specific weight of the sediment in fluid, $d$ is the grain diameter, and the dimensionless constant $\psi_{c}$ is the critical Shields parameter. Experimental work by Shields (in Yalin 1977) indicates $\psi_{c}=0.05 \pm 0.01$. Combining (3.2.4) and (3.2.5) by setting $\tau^{\prime}=$ $\tau_{c}^{\prime}$ finally gives the following upper bound on equilibrium cross-sectional geometry as a function of discharge and other "independent" variables describing sediment and roughness characteristics:

$$
A h_{R}^{1 / 6}=Q n\left(\frac{\rho g}{\tau_{c}}\right)^{1 / 2}
$$

where the total critical shear stress, $\tau_{c}$, is given by

$$
\tau_{c}=\psi_{c} \rho g G d\left(\frac{\tau}{\tau^{\prime}}\right)
$$

\subsubsection{Comparison to observations}

To compare (3.2.6) to the observations in Figure 3.1, several additional assumptions are necessary. The density of the sediments is assumed to be well represented by quartz, for which $G=1.65$. Because of the large width-to-depth ratio of the channels, it is reasonable to equate $h_{R}$ to the mean depth of the cross-section, $h$. Errors in $Q$ are estimated to be $20 \%$, whereas $A$ and $h$ are assumed accurate to within 5\%. Likely variance in $n$ and $\tau^{\prime} / \tau$ are somewhat larger, as discussed in the previous section. However the least constrained variable is $d$.

Table 3.3 lists the few sources from Table 3.1 which provide bottom sediment information. Four sources indicate fine-to-medium sand bottoms, one indicates cohesive mud, and one indicates bottom sediment which is "highly organic and black and has a texture not immediately obvious in the field" (Myrick and Leopold 1963, p.4). Of course (3.2.7) is not relevant to mud bottoms, for which the issue of cohesion must be addressed, and likewise for "highly organic" sediment. In applying (3.2.6) - (3.2.7) to the four 
systems with bottom sediments that are known to be non-cohesive, reasonable approximations for $d$ are used (Table 3.3). Otherwise, $d=2 \pm 1$ phi is chosen, where phi $=$ $-\log _{2}(d$ in $\mathrm{mm})$. This choice is centered at $d=0.25 \mathrm{~mm}$, and includes the range typically defined as fine to medium sand $(1 / 8$ to $1 / 2 \mathrm{~mm})$. Using $d=2 \mathrm{phi}$, along with $\rho=10^{3}$ $\mathrm{kg} \mathrm{m}^{-3}$ and the previously discussed values for $\psi_{c}, G$ and $\tau^{\prime} / \tau$, gives $\tau_{c}=0.5 \mathrm{~N} \mathrm{~m}^{-2}$ with upper and lower error bounds of $0.1 \mathrm{~N} \mathrm{~m}^{-2}$ and $1.1 \mathrm{~N} \mathrm{~m}^{-2}$, respectively.

If critical shear stress theory is applied to cohesive sediment, a first-order result is given by evaluating (3.2.4) using the magnitude of critical erosion shear stress $\left(\tau_{e}\right)$ typically observed above mud bottoms (e.g., Partheniades 1965; Dyer 1986). According to Dyer (1986), the thin layer of loosely held mud flocs typically found at the surface of quiescent mud bottoms generally erodes at $\tau \approx 0.1 \mathrm{~N} \mathrm{~m}^{-2}$. Once this layer has been suspended, the underlying mud deposit generally has a $\tau_{e}$ of about $1 \mathrm{~N} \mathrm{~m}^{-2}$. Setting $\tau=\tau_{e}$ in (3.2.4) gives the following expression for cross-sectional geometry:

$$
A h_{R}^{1 / 6}=Q n\left(\frac{\rho g}{\tau_{e}}\right)^{1 / 2}
$$

In evaluating (3.2.8), $n$ is again chosen to be $0.03 \pm 0.005 \mathrm{~m}^{-1 / 3} \mathrm{~s}$, since the application of Manning's $n$ to natural channels appears to be insensitive to bottom sediment type within the mud to sand range (Henderson 1966). Partheniades (1965) found rapid erosion of San Francisco Bay mud occurred after a critical shear stress of about 0.5 to 1.3 $\mathrm{N} \mathrm{m}^{-2}$ had been exceeded. Thus $\tau_{e}$ is chosen here to equal $0.9 \pm 0.4 \mathrm{~N} \mathrm{~m}^{-2}$. Equation (3.2.8) admittedly neglects the role of bedform drag. However the value of $\tau^{\prime} / \tau$ applied to (3.2.6) - (3.2.7) is for non-cohesive sand only and cannot confidently be used in deriving (3.2.8).

Figures 3.2 and 3.3 display (3.2.6) and (3.2.8), along with error bounds, superimposed on the field observations. The propagation of normally distributed, random error in (3.2.6) and (3.2.8) is determined by the relation (e.g., Young 1962):

$$
\Delta f^{2}=\left(\frac{\partial f}{\partial a}\right)^{2} \Delta a^{2}+\left(\frac{\partial f}{\partial a}\right)^{2} \Delta b^{2}+\ldots
$$

where $\Delta f$ is the error in $f, \Delta a$ and $\Delta b$ are the errors in $a$ and $b$, and $f=f(a, b, \ldots)$. For the error bounds in curves in Figure 3.2, uncertainty is considered in both the abscissa and the ordinate. Thus

$$
f=\frac{A h_{R}^{1 / 6}}{Q n}\left(\frac{\tau_{c}}{\rho g}\right)^{1 / 2}
$$


Applying (3.2.7) then gives

$$
\left(\frac{\Delta f}{f}\right)^{2}=\left(\frac{\Delta A}{A}\right)^{2}+\frac{1}{6}\left(\frac{\Delta h_{R}}{h_{R}}\right)^{2}+\left(\frac{\Delta Q}{Q}\right)^{2}+\left(\frac{\Delta n}{n}\right)^{2}+\frac{1}{2}\left(\frac{\Delta \tau_{c}}{\tau_{c}}\right)^{2} .
$$

The error analysis for Figure 3.3 is identical, replacing $\tau_{e}$ for $\tau_{c}$.

Within error bounds, (3.2.6) roughly predicts the geometric parameter $A h_{R}{ }^{1 / 6}$ as a function of $Q$ and the other "independent" variables (Figure 3.2). More than half of the cross-sections fall within the error bars of (3.2.6). However most of the sections fall below the line predicted by (3.2.6), and many fall entirely below the range of the likely error. As was earlier emphasized, critical shear stress theory only provides a lower bound on $\tau_{s}$ and, therefore, an upper bound on equilibrium geometry. It is reassuring to note that none of the observations in Figure 3.2 fall above the error bounds.

The error bounds on (3.2.8) encompass fewer of the observations (Figure 3.3). Again, available information (Table 3.3 ) suggests the majority of the tidal channels in Table 3.1 are not formed in cohesive mud. However (3.2.8) also under-predicts $A h_{R}{ }^{1 / 6}$ for many cross-sections, including most of those known to be floored by cohesive material (Figure 3.3). Thus $\tau_{e}$ may be an overestimate of the $\tau_{s}$ appropriate to some mud channels. Except for the error bounds, (3.2.6) and (3.2.8) produce similar curves. This explains why (3.2.6) in Figure 3.2 also encompasses the cross-sections known to be formed in cohesive material.

Thus critical shear stress theory provides a reasonable upper bound for the observed relationship between peak discharge and cross-sectional geometry of tidal channels, at least for channels in non-cohesive sediment. For both cohesive and noncohesive sediment, the theory predicts that at equilibrium, tidal channel geometry will follow the relation

$$
A h_{R}^{1 / 6} \sim Q
$$

If regressions of the form $A h_{R}{ }^{1 / 6} \sim Q^{\beta}$ are applied to the individual systems in Table 3.1, then the mean value for $\beta$ is $: .01 \pm 0.04$ (Table 3.4). This value is indistinguishable from one and, therefore, consistent with critical shear stress theory. 
3.3 Deviations of stability shear stress from critical shear stress

\subsubsection{Deviations among channels}

Cross-sections from systems having small $Q$ fall about evenly on either side of (3.2.6) and (3.2.7) in Figures 3.2 and 3.3. However cross-sections with larger $Q$ fall consistently below the theoretical curves. This qualitative trend is confirmed statistically if a least-squares regression of the form $A h_{R}{ }^{1 / 6} \sim\left\{Q \tau_{c}{ }^{-1 / 2}\right\}^{\beta}$ is performed on all the crosssections at once. ( $\tau_{c}^{-1 / 2}$ is included in the regression because $\tau_{c}$ can vary between systems as a function of $d$.) The regression shows $\beta=0.90 \pm 0.01$, rather than $\beta=1$ as predicted by critical shear stress theory. Yet the mean along-channel value for $\beta$ is statistically indistinguishable from one (Table 3.4). This supports a second form of $\tau_{s}$ theory, namely that $\tau_{s}$ tends to be uniform throughout any one channelized tidal embayment, but may have a value greater than that required for initiation of sediment motion.

Even though large $Q$ is associated with over-prediction of $A h_{R}^{1 / 6}$ in Figures 3.2 and 3.3, $Q$ is probably not the variable directly responsible for the observed deviation. If the misfit were directly a result of increasing $Q$, then along-channel variations in $Q$, which can be several orders of magnitude, should also cause $\beta<1$. Here it is postulated that among different systems, consistent deviations from a single theoretical curve are largely due to differences in spring tidal range. Unlike $Q, R_{s p}$ is nearly constant throughout individual systems and is therefore compatible with uniform along-channel behavior of $A h_{R}{ }^{1 / 6}$. Thus the population-wide $Q$-dependent deviation may largely be the result of a fortuitous correlation between $Q$ and $R_{s p}$.

Figure 3.4 displays mean (log-space) deviations from (3.2.6) as a function of $R_{s p}$ for each system in Figure 3.2 along with the best-fit log-log least-squares regression. The best-fit curve with standard errors is (with $R_{s p}$ in meters):

$$
\frac{A h_{R}^{1 / 6}}{Q n}\left(\frac{\tau_{c}}{\rho g}\right)^{1 / 2}=(0.77 \pm 0.13) R_{s p}-0.39 \pm 0.14
$$

Or equivalently,

$$
A h_{R}^{1 / 6}=Q n\left(\frac{\rho g}{\tau_{s}}\right)^{1 / 2}
$$

where

$$
\tau_{s}=(1.7 \pm 0.4) R_{s p}^{0.8 \pm 0.3} \tau_{c}
$$


Since $\tau_{c}$ has already been shown to provide a reasonable lower bound on $\tau_{s}$, it is sensible to apply (3.3.3) only to those systems with tidal ranges large enough to give $\tau_{s} \geq \tau_{c}$. Thus (3.3.3) applies only to systems with $R_{s p}>\sim 0.5 \mathrm{~m}$. Tidal channels with $R_{s p} \leq 0.5 \mathrm{~m}$ can be expected to have a system-wide $\tau_{s}$ about equal to $\tau_{c}$. For systems with $R_{s p}>0.5$, however, system-wide $\tau_{s}$ typically increases as a function of $R_{s p}$.

Figure 3.5 displays (3.3.2) - (3.3.3) superimposed on all of the field observations, with error bounds again determined via (3.2.9). The observations in Figure 3.5 fall about evenly on either side of (3.3.2), suggesting (3.3.2) provides a reasonable mean value for cross-sectional geometry as a function of discharge, not just an upper bound. If a regression of the form $A h_{R}{ }^{1 / 6} \sim\left\{Q \tau_{s}^{-1 / 2}\right\}^{\beta}$ is performed on all the cross-sections, the result is $\beta=0.95 \pm 0.01$, which is significantly closer to one. Propagation of error in (3.3.2) (3.3.3) is a function of tidal range, which cannot be shown directly on Figure 3.5. The error bounds in Figure 3.5 are therefore determined by averaging the error bounds given by (3.3.2) - (3.3.3) for each observed tidal range.

Stability shear stress may vary with $R_{s p}$ partly due to the nature of the analysis used in this study. It is possible that the methods used overestimate the value of $Q$ most relevant to equilibrium morphology in channels with large $R_{s p}$ and underestimate the most relevant $Q$ in those with small $R_{s p}$. First, the ratio of spring to mean tidal range generally increases with tidal range. On the Potomac $\left(R_{s p}=1 \mathrm{~m}\right)$ spring range is only $10 \%$ greater than the mean range, whereas on the Tamar $\left(R_{s p}=4.7 \mathrm{~m}\right)$ it is $36 \%$ greater, and on the Usk $\left(R_{s p}=\right.$ $12 \mathrm{~m}$ ), it is $45 \%$ greater. If the most relevant discharge is actually a weighted average of all discharges, then use of spring discharge alone will tend to overestimate $\tau_{s}$ in channels with large tide ranges. Second, systems having small tide ranges may be more sensitive to morphologic change by non-tidal forces. The occasional flood or storm surge is more likely to overflow the banks of a smaller tide range channel and cause more severe erosion. Neglecting non-tidal forces may underestimate the most relevant $Q$ (and thus underestimate $\left.A h_{R}{ }^{1 / 6}\right)$ in channels with small tide ranges.

Stability shear stress may also be a true function of spring tidal range. Salt marshes, which often act as sediment traps, are sensitive to tide range and generally do not extend below about one meter of the mean high water line (Frey and Basan 1985). The larger the tidal range, the smaller the relative area of sediment-trapping marsh and the larger the likely expanse of exposed sediment in intertidal flats. These properties may result in a greater sediment supply to the channels. The relative "clogging" of the channel with 
sediment may decrease cross-sectional area and increase $U=Q / A$ until a $\tau_{s}>\tau_{c}$ is reached which can disperse the sediment as fast as it is supplied. Tide range is also associated with characteristic patterns of tidal distortion, which, in turn, should favor increased or decreased channel cross-sectional area at equilibrium. All else being equal, channels with small tide ranges tend to be ebb-dominant, whereas channels with large tide ranges tend to be flood-dominant (Friedrichs and Aubrey 1988; Chapter 2 of this thesis). Ebb-dominant channels will tend to flush sediment out of a system more effectively, decreasing the level of $\tau_{s}$ otherwise needed to prevent shoaling. Flood-dominant systems will tend to trap sediment within a tidal channel, increasing the needed level of $\tau_{s}$.

\subsubsection{Along-channel deviation}

An assumption of uniform $\tau_{s}$ along the length of individual tidal channels leads to the relation $A h_{R}{ }^{1 / 6} \sim Q$. Along-channel deviations from $A h_{R}{ }^{1 / 6} \sim Q$ are measured by a least-squares fit of $A h_{R}{ }^{1 / 6} \sim Q^{\beta}$ to the cross-sectional data from a single system. If $\beta>1$, $A h_{R}{ }^{1 / 6}$ will be larger than predicted near the seaward end of the channel where $Q$ is high and smaller than predicted near the landward end of the channel where $Q$ is low. If the channel is stable, then $\beta>1$ also implies that $\tau_{s}$ decreases in a seaward direction. If $\beta<1$, then the opposite is true, and $\tau_{s}$ decreases in a landward direction. In the previous section it was suggested that ebb- or flood-dominant discharge could cause an entire system to have a higher or lower $\tau_{s}$. In this section it is suggested that along-channel variations in discharge asymmetry can lead to along-channel variations in $\tau_{s}$.

If the seaward portion of a tidal channel is flood-dominant while the landward portion is ebb-dominant, then a spatial convergence in the direction of maximum discharge will cause a localized increase in sediment concentration. This process has been documented previously as a "tidal turbidity maximum" in such tidal rivers as the Gironde in France (Allen et al. 1980) and the Tamar in the U.K. (Uncles and Stephens 1989; Stephens et al. 1992). Seaward of the turbidity maximum, flood-dominance brought about by a large tidal range-to-depth ratio favors landward movement of sediment; landward of the turbidity maximum, ebb-dominance brought about by fresh water discharge favors seaward movement of sediment. The resulting turbidity maximum is observed to migrate alongchannel as seasonal variations in fresh water discharge cause displacement of the convergence point .

Both Allen et al. (1980) and Stephens et al. (1992) used 1-D numerical models to study the hydrodynamics associated with tidally-induced turbidity maxima. When using 
realistic along-channel geometries from the Gironde and the Tamar, both of their models predicted a localized increase in maximum shear stress associated with a rapid constriction in cross-sectional area. In each case, maximum shear stress was predicted to decrease landward and seaward of this point. For both the Gironde and the Tamar this fixed region of increased shear stress was found to be in the general vicinity of the previously observed, migrating turbidity maximum. Both studies suggested that this local increase in shear stress may enhance resuspension in the vicinity of the migrating turbidity maximum.

Here it is argued that the tidal turbidity maxima and locally increased stress are morphodynamically related and may help explain observed deviations from $\beta=1$ along some stable tidal channels. Evolution towards equilibrium might proceed as follows: First, an along-channel switch from ebb- to flood-dominance favors collection of sediment at a tidal turbidity maximum. Then deposition at the turbidity maximum reduces crosssectional area, locally increasing $U=Q / A$ and, therefore, increasing maximum bottom shear stress. Ultimately, $A$ is decreased until a $\tau_{s}$ is reached which prevents further deposition and effectively disperses sediment as fast as it is supplied. At equilibrium, $\tau_{\boldsymbol{s}}$ will decrease both seaward and landward from the transition from flood- to ebbdominance.

If a stable channel is examined seaward of the switch from ebb- to flooddominance, then a seaward decrease in $\tau_{s}$ should cause $\beta>1$ and be associated with flooddominant discharge. Conversely, a decrease in $\tau_{s}$ landward of the transition should cause $\beta$ $<1$ and be associated with ebb-dominance. The larger the tidal range, the farther inland this transition from $\beta>1$ to $\beta<1$ should occur, and the more likely a fit to all the crosssections will give $\beta>1$. These trends seems to borne out by Table 3.4 which indicates that the six channels with $R_{s p}>4 \mathrm{~m}$ all have $\beta \geq 1$. Of these six channels, sufficient information is available to calculate ratios of flood-to-ebb peak discharge $\left(Q_{\text {flood }} / Q_{e b b}\right)$ along the Ord, Western Scheldt and Tamar. Average values for $Q_{f l o o d} / Q_{e b b}$ at sections along these three tidal channels are 2.0, 1.5 and 1.2, respectively, confirming an association of flood-dominance with $\beta>1$. The relatively low value of $\beta=1$ for the high tide range Thames $\left(R_{s p}=5.2\right)$ may be due to a sampling of cross-sections on both sides of the transition from flood- to ebb-dominance. Figure 5.6 displays $A h_{R}{ }^{1 / 6}$ as a function of $Q$ for the Thames as predicted by (3.3.2) - (3.3.3). A least-squares fit of $A h_{R}{ }^{1 / 6} \sim Q^{\beta}$ to the seven most seaward sections gives $\beta>1$, while a fit to the three most landward sections gives $\beta<1$. 
All of the tidal channels subject to tides of mixed type (San Francisco and the three Oregon channels), have $\beta<1$ (Table 3.4). This may be due to a systematic association of mixed tides with ebb-dominance. In a mixed-tide regime, the lower low tide usually follows the higher high tide, causing the largest changes in tidal elevation to occur consistently during the ebb. However this consideration is merely speculative. The unusually low values for $\beta$ in the Oregon channels could also result from choking of the seaward end by littoral drift even beyond the most seaward cross-section (the seawardmost section of each Oregon channel has already been dropped from the analysis).

\subsection{Application to embayments}

In this section uniform along-channel $\tau_{s}$ theory is used to derive equilibrium alongchannel geometries for entire embayments. If the embayment length is much less than the tidal wavelength, then continuity of fluid flow may be applied kinematically to determine along-channel discharge as a function of intertidal storage in areas such as tidal flats or marshes. A known distribution of along-channel discharge then can be combined with results from the previous section relating $A h_{R}^{1 / 6}$ to $Q$. In the following sub-sections relations for embayments with exponential and power-law storage areas are first derived theoretically and then compared to observations from actual exponential and power-law embayments, namely Wrecked Recorder Creek, Virginia (Myrick and Leopold 1963) and the Ord River in Western Australia (Wright et al. 1973).

\subsubsection{Theory}

In tidal channels where the length of the tidal wave is much greater than the length of the channel, spring tidal elevation $(\zeta)$ and discharge $(q)$ at the dominant tidal frequency are reasonably described by

$$
\zeta=Z(x) \cos \omega t \text {, and } q=Q(x) \sin \omega t
$$

where $\omega=2 \pi / T$, and $x=0$ at the landward end of the embayment. Any contributions from fresh water discharge are neglected. The linearized continuity equation for channelized flow in tidal embayments with intertidal storage in flats or marsh is given by

$$
b \frac{\partial \zeta}{\partial t}+\frac{\partial q}{\partial x}=0
$$


where $b$ is the time-averaged total system width (including storage regions), and $q$ is confined to the central tidal channel. Substituting (3.4.1) into (3.4.2) then gives

$$
b Z \omega=\frac{\mathrm{d} Q}{\mathrm{~d} x}, \text { or } Q=\omega \int_{0}^{x} b Z \mathrm{~d} x^{\prime}
$$

When evaluating (3.4.3), tidal embayments are considered with observed $b(x)$ and $Z(x)$ which may be crudely described either by a power law, i.e.,

$$
b=b_{L}\left(\frac{x}{L}\right)^{\sigma_{b}}, \text { and } Z=Z_{L}\left(\frac{x}{L}\right)^{\sigma_{z}}
$$

or by an exponential relation, i.e.,

$$
b=b_{0} \mathrm{e}^{\lambda_{b} x / L}, \text { and } Z=Z_{0} \mathrm{e}^{\lambda_{2} x / L}
$$

(Figure 3.7). The subscripts 0 and $L$ indicate values at $x=0$ and $x=L$, and $\sigma_{b}, \lambda_{b}, \sigma_{z}$ and $\lambda_{z}$ are dimensionless constants describing the rate of expansion of the storage area and rate of growth of tidal amplitude with distance.

Introducing (3.4.4) and (3.4.5) into (3.4.3b) and integrating gives

$$
Q=\frac{\omega L Z_{L} b_{L}}{\left(\sigma_{b}+\sigma_{z}+1\right)}\left(\frac{x}{L}\right)^{\left(\sigma_{b}+\sigma_{z}+1\right)}
$$

for power-law embayments, and

$$
Q=\frac{\omega L Z_{0} b_{0}}{\left(\lambda_{b}+\lambda_{z}\right)}\left\{e^{\left(\lambda_{b}+\lambda_{z}\right) x / L}-1\right\}
$$

for those following an exponential relation. Finally, (3.3.2) is used to eliminate $Q$, yielding

$$
A h_{R}^{1 / 6}=n\left(\frac{\rho g}{\tau_{s}}\right)^{1 / 2} \frac{\omega L Z_{L} b_{L}}{\left(\sigma_{b}+\sigma_{z}+1\right)}\left(\frac{x}{L}\right)^{\left(\sigma_{b}+\sigma_{z}+1\right)}
$$

and

$$
A h_{R}^{1 / 6}=n\left(\frac{\rho g}{\tau_{s}}\right)^{1 / 2} \frac{\omega L Z_{0} b_{0}}{\left(\lambda_{b}+\lambda_{z}\right)}\left\{\mathrm{e}^{\left(\lambda_{b}+\lambda_{z}\right) x / L-1}\right\}
$$

for power-law and exponential systems, respectively. 


\subsubsection{Comparison to observations}

Observations from Wrecked Recorder Creek (Figure 3.8) and the Ord River (Figure 3.9) are chosen for comparison to (3.4.6) - (3.4.9) because of the detailed morphologic information available (Myrick and Leopold 1963; Wright et al. 1973) and also because of their reasonable correspondence to idealized power-law and exponential relations for intertidal storage area. Figure 3.10 displays best fits of (3.4.4) and (3.4.5) to observations of $b$ and $Z$ as a function of along-channel distance. Values for $Z$ along the Ord are observed $\mathrm{M}_{2}$ amplitude scaled by the ratio $R_{s p} / R_{o b s}$ (see Table 3.1). Wrecked Recorder Creek is so short that $Z$ is constant along channel, and $\sigma_{2}=0$.

Time-averaged widths are calculated as $b=\left(b_{S H W}+b_{S L W}\right) / 2$, where $S H W$ and $S L W$ indicate spring high and low water. Estimates of $b_{S H W}$ are made by dividing the surface area between each "storage segment boundary" in Figures 3.8 and 3.9 by the length of each channel segment. $b_{S L W}$ is assumed equal to the average value of $w$ for each segment. The areal extent of tidal marsh drainage into Wrecked Recorder Creek (Figure 3.8) is inferred from the positions of neighboring tidal Creeks also depicted by Myrick and Leopold (1963). The areal extent of intertidal flats adjacent to the Ord is clearly shown by Wright et al. (1973).

Best-fit values for the geometric parameters in (3.4.4) - (3.4.5) are $b_{0}=430 \pm 120$ $\mathrm{m}, \lambda_{b}=3.7 \pm 0.4, Z_{0}=0.63 \pm 0.23 \mathrm{~m}$ and $\lambda_{2}=1.6 \pm 0.5$ for the Ord River, and $b_{L}=210 \pm 30$ $\mathrm{m}$ and $\sigma_{b}=0.17 \pm 0.10$ for Wrecked Recorder Creek. $Z_{L}$ for Wrecked Recorder Creek is assumed to equal to $R_{s p} / 2$. Figure 3.11 shows (3.4.6) and (3.4.7) superimposed on observations of $Q$ as a function of distance along the Ord and Wrecked Recorder Creek. The $\mathrm{M}_{2}$ tidal period is used to calculate $\omega$, and propagation of error is once more calculated via (3.2.9). The excellent agreement confirms that the intertidal storage areas of these systems may be represented adequately by a simple power-law or exponential relations. The minimal scatter in Figure 3.11 should not be too surprising, however. Because $Q(x)$ is an integration of $b(x), Q(x)$ will naturally be "smoother" than $b(x)$.

In order to compare (3.4.8) and (3.4.9) to observations, $\tau_{s}$ must first be estimated. From (3.2.7) and (3.3.3),

$$
\tau_{s} \approx 1.7 R_{s p}^{0.8} \psi_{c} \rho g G\left(\frac{\tau}{\tau^{\prime}}\right)
$$


To evaluate (3.4.10), the same values for $\psi_{c}, \rho, G$ and $\tau / \tau$ that were used in earlier sections are applied once more. Then the relatively large tidal range $\left(R_{s p}=5.9 \mathrm{~m}\right)$ and coarse sediments $(d \approx 0.35 \mathrm{~mm})$ in the Ord give a prediction of $\tau_{s} \approx 5 \mathrm{~N} \mathrm{~m}^{-2}$ with lower and upper bounds of 1 and $10 \mathrm{~N} \mathrm{~m}^{-2}$. The smaller tide range at Wrecked Recorder Creek $\left(R_{s p}=1 \mathrm{~m}\right.$ ) and unknown sediment properties (i.e., let the equivalent $d \approx 0.25 \mathrm{~mm}$ ) lead to a best guess of $\tau_{s}=0.9 \mathrm{~N} \mathrm{~m}^{-2}$ with lower and upper bounds of 0.2 and $1.9 \mathrm{~N} \mathrm{~m}^{-2}$.

Figure 3.12 shows plots of $A h_{R}^{1 / 6}$ predicted by (3.4.8) - (3.4.9) superimposed on observations from the Ord and Wrecked Recorder Creek. The observed cross-sectional geometries as a function of distance along-channel are well within error bars and, therefore, are consistent with the predictions of uniform $\tau_{s}$ theory. The error bars in Figure 3.12 are based solely on uncertainty in the prediction of $\tau_{s}$ by (3.4.10). Uncertainty in the estimates of $Q(x)$ and $n$ have been purposely excluded to emphasize the huge possible range in $\tau_{s}$. More accurate predictions of $\tau_{s}$ are not possible without further constraints on such variables as sediment type, form drag, and the correlation of $\tau_{s}$ with tidal range. Though the likely magnitude of any system-wide $\tau_{s}$ is poorly constrained, the successful reproduction of the form of along-channel variation in $A h_{R}{ }^{1 / 6}$ supports the concept of a system-wide nearly uniform $\tau_{s}$. Further work is warranted to understand better the large variations in characteristic $\tau_{s}$ among different tidal channels.

\subsection{Further implications of uniform stability shear stress}

This study demonstrates the tendency of tidal channels to minimize along-channel variations in $U$ by morphodynamically adjusting toward a uniform distribution of $\tau_{s}$. Uniform $U$ and $\tau$ have important ramifications concerning classical views of mass and momentum balances, mechanisms for net sediment transport, and formulations of alongchannel mixing coefficients.

Morphologic equilibrium directly affects the appropriate scaling of the continuity equation given by (3.4.2). When modeling channelized tidal embayments analytically, it is common practice to assume a prismatic channel and express continuity as

$$
b \frac{\partial \zeta}{\partial t}+A \frac{\partial u}{\partial x}=0
$$


However, a channel near morphologic equilibrium will have nearly uniform along channel velocity. Assuming the length of the tidal wave is much greater than the length of the tidal channel, then (3.4.2) should be approximated more properly as

$$
b \frac{\partial \zeta}{\partial t}+u \frac{\mathrm{d} A}{\mathrm{~d} x}=0
$$

The hydrodynamic implications of (3.5.2) are discussed in Chapter 4 of this thesis.

In the momentum equation, uniform $U$ obviously minimizes the contribution of advective acceleration to the momentum balance. In fact, the scaling which allows formulation of continuity as (3.5.2) also indicates that local acceleration must be much smaller than the pressure gradient (see Chapter 4 ). The only term left in the 1-D momentum equation which can balance pressure at lowest order is friction. The result is a zero-inertia equation in momentum that, when combined with (3.5.2), yields a first-order wave equation for elevation or velocity. This equation and the form of its solutions diverge from the classical wave equation for tides and the conventional view of tidal co-oscillation (see Chapter 4).

Uniform $\tau_{s}$ is also affects classical models for net sediment transport based on "scour lag" and "settling lag". According to Postma (1961), as a waning current falls below the speed necessary for sediment suspension, a sediment particle will continue to travel landward because it takes some time for the particle to reach the bottom (settling lag). After the turn of the tide, the particle will not be re-suspended until later in the tidal cycle because the bottom shear necessary for resuspension is significantly higher than that necessary for suspension (scour lag). As long as there is a landward decrease in $\tau$, these mechanisms will result in net landward sediment transport. If $\tau$ does not decrease systematically in a landward direction, however (and uniform $\tau_{s}$ theory suggests it often does not), then settling lag and scour lag will be about the same on the ebb and the flood, and these lags will not cause net sediment transport.

Uniform $\tau_{s}$ also impacts the analytic prediction of eddy diffusivities based on the distribution of tidal currents and tidal excursions. In tidal estuaries, the eddy diffusivity, $K$, has been predicted to be proportional to the tidal excursion, $L_{e}$, times the velocity amplitude, $U$ (Arons and Stommel 1951). If a short tidal channel is assumed to be prismatic, then $U$ and $L_{e}$ will both be proportional to $x / L$, and, therefore, $K \sim(x / L)^{2}$ (Arons and Stommel 1951). If the tidal channel is near equilibrium, however, $U$ and $L_{e}$ 
will be nearly uniform. Thus $K \sim U L_{e}$ should be nearly constant along an equilibrium channel, rather than proportional to $(x / L)^{2}$.

\subsection{Summary and conclusions}

A survey of the literature allows estimates of peak spring discharge and crosssectional geometry at 146 sections in 18 separate tidal systems. Previous explanations for the near proportionality of cross-sectional area $(A)$ and discharge $(Q)$ include maximum entropy, uniform critical velocity, and a propensity toward plane bed flow. The purpose of this study was to relate channelized tidal embayment morphology to flow properties via a more robust, physical mechanism, namely the stability shear stress $\left(\tau_{s}\right)$ just necessary to maintain a zero gradient in net along-channel sediment transport. It is assumed that if $\tau>$ $\tau_{s}$, net erosion will occur, increasing $A$, and reducing $\tau \sim(Q / A)^{2}$ back toward $\tau_{s}$. If $\tau<\tau_{s}$ there will be net deposition, reducing $A$ and increasing $\tau$ toward $\tau_{s}$.

A theoretical lower bound on $\tau_{s}$ (and an upper bound on $A$ ) is provided by the condition $\tau^{\prime}=\tau_{c}^{\prime}$, where $\tau^{\prime}$ is maximum grain shear stress and $\tau_{c}^{\prime}$ is the critical grain shear stress necessary for initiation of sediment motion. Critical shear stress theory is applied to equilibrium tidal channel geometry by (i) relating $U=Q / A$ to $\tau$ via the Manning-Strickler equation, (ii) relating total shear stress, $\tau$, to $\tau$ ' via empirical ratios observed in the literature, and (iii) determining $\tau^{\prime}=\tau_{c}^{\prime}$ from the Shields criterion for the initiation of sediment motion. For cohesive sediments, $\tau$ is assume to equal $\tau_{e}$ at equilibrium, where $\tau_{e}$ is the magnitude of critical erosion shear typically observed above mud bottoms.

Comparison to observations indicates $\tau^{\prime}=\tau_{c}^{\prime}$ does a reasonable job of predicting equilibrium cross-sectional geometry in general and an excellent job of predicting the upper bound on likely geometry. Error bars on likely geometry are large due to uncertainties in sediment grain diameter and in the appropriate value for $\tau^{\prime} / \tau$. Except for error bounds, $\tau^{\prime}=$ $\tau_{c}^{\prime}$ and $\tau=\tau_{e}$ produce similar curves. In either case, uniform critical values for $\tau$ predict that at equilibrium, along-channel geometry will follow the relation $A h_{R}{ }^{1 / 6} \sim Q$, where $h_{R}$ is the hydraulic radius. Along-channel regressions of the form $A h_{R}{ }^{1 / 6} \sim Q^{\beta}$ give a mean observed value for $\beta$ of $1.01 \pm 0.04$, which is indistinguishable from one and, therefore, consistent with critical shear stress theory.

Although along-channel geometry agrees, on average, with the prediction $A h_{R}^{1 / 6} \sim$ $Q$, the uniform $\tau_{s}$ appropriate to individual systems can vary widely above that predicted by $\tau^{\prime}=\tau_{c}^{\prime}$. Observed $\tau_{s}$ is found to vary among systems according to the relation $\tau_{s} \approx 1.7$ 
$R_{s p}^{0.8} \tau_{c}$, where $R_{s p}$ (in meters) is spring tidal range, and $\tau_{c}$ is the total shear stress when $\tau^{\prime}=\tau_{c}^{\prime} . \tau_{s}$ may vary with $R_{s p}$ because of the decrease in sediment-trapping vegetation and increase in exposed flats associated with large $R_{s p}$. An increased sediment supply may "clog" the channel, increasing the $\tau_{s}$ necessary for effective sediment dispersal. Also, small $R_{s p}$ favors ebb-dominance, whereas large $R_{s p}$ favors flood-dominance. Ebbdominance may aid the flushing of sediment, decreasing $\tau_{s}$, whereas flood-dominance may enhance shoaling and increase $\tau_{s}$.

Observed deviations from $A h_{R}^{1 / 6} \sim Q$ along individual channels are associated with a convergence in discharge asymmetry. It is hypothesized that a spatial convergence in the direction of maximum discharge may cause net deposition, a reduction in $A$, and a local increase in $U=Q / A$ until a $\tau_{s}$ is reached which prevents further deposition. In a stable channel, $\tau_{s}$ will then decrease both seaward and landward of the convergence point. If a regression of the form $A h_{R}{ }^{1 / 6} \sim Q^{\beta}$ is applied along such a channel, one should find $\beta>1$ associated with flood-dominance landward of the maximum in $\tau_{s}$ and $\beta<1$ associated with ebb-dominance seaward. Geometries and discharge asymmetries along several channels are observed to be consistent with this pattern.

An assumption of uniform along-channel $\tau_{s}$ is used to derive equilibrium alongchannel geometries for entire embayments. Assuming the embayment to be short relative to the tidal wavelength, continuity of fluid flow gives along-channel $Q$ as a function intertidal storage in flats and marsh. Previously derived relations between $A h_{R}{ }^{1 / 6}$ and $Q$ then predict equilibrium $A h_{R}{ }^{1 / 6}$ as a function of distance along channel. Theoretical predictions are consistent with published observations from Wrecked Recorder Creek, Virginia, and the Ord River in Westem Australia.

Finally, consideration of uniform $\tau_{s}$ calls into question classical models for firstorder mass and momentum balances, likely mechanisms for net sediment transport, and analytic expressions for along-channel mixing coefficients commonly applied to real tidal channels. 
Table 3.1. Data for tidal channels and sheltered inlets.

Location

Source

\# of

$R_{o b s}$

$R_{s p}$

$A \sim Q^{\alpha}$

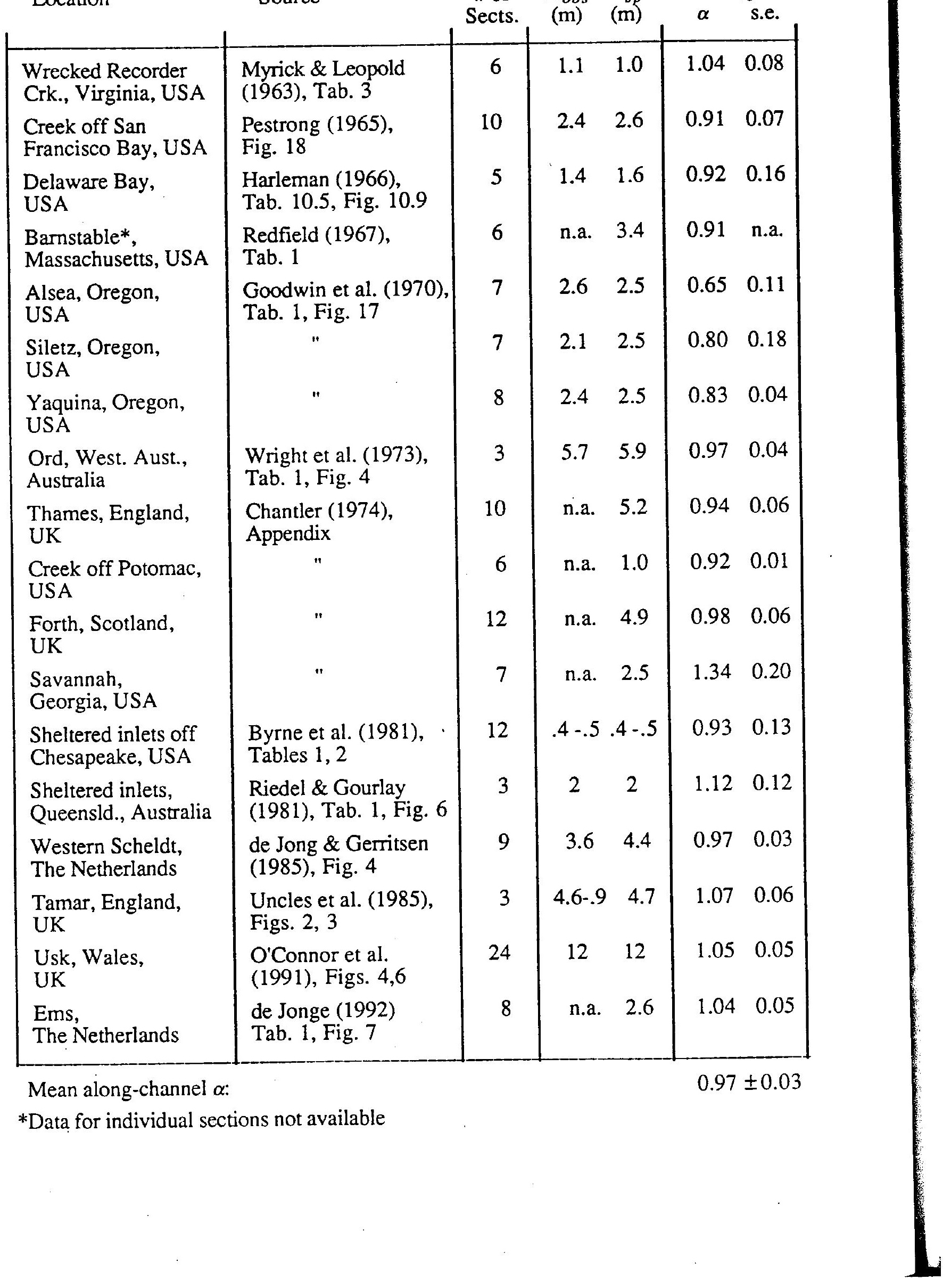


Table 3.2. Ratio of grain to total shear stress over naturally formed ripples and dunes.

\begin{tabular}{|c|c|c|c|}
\hline Location & Source & $\begin{array}{l}\text { Mean } d \\
(\mathrm{~mm})\end{array}$ & $\tau^{\prime} / \tau$ \\
\hline & & & \\
\hline Laboratory flume & Bagnold (1963) & $0.2-0.7$ & $0.2-0.5$ \\
\hline Columbia River & $\begin{array}{c}\text { Smith (1977) and } \\
\text { Smith \& McLean (1977) }\end{array}$ & 0.27 & $0.18-0.24$ \\
\hline Laboratory flume & $\begin{array}{c}\text { Engelund \& Fredsoe (1982) } \\
\text { and Fredsoe (1982) }\end{array}$ & $0.2-0.9$ & $0.3-0.5$ \\
\hline " & Paola (1983) & 0.2 & $0.58-0.65$ \\
\hline$"$ & Kapdasli \& Dyer (1986) & $0.14-0.5$ & $0.08-0.17^{*}$ \\
\hline
\end{tabular}

*Observation over ripple crest. Other four observations are spatially averaged. 
Table 3.3. Channel bottom sediment type at cross-sections.

Location

Source

Sediment type

Median phi

\begin{tabular}{|c|c|c|c|}
\hline $\begin{array}{l}\text { Wrecked Recorder } \\
\text { Crk., Virginia, USA }\end{array}$ & $\begin{array}{l}\text { Myrick \& Leopold } \\
\text { (1963) }\end{array}$ & "highly organic" & n.a. \\
\hline $\begin{array}{l}\text { Creek off San } \\
\text { Francisco Bay, USA }\end{array}$ & Pestrong (1965) & mud & 7.8 \\
\hline $\begin{array}{l}\text { Ord, West. Aust., } \\
\text { Australia }\end{array}$ & Wright et al. (1973) & medium fine sand & $1.5 \pm 0.5$ \\
\hline $\begin{array}{l}\text { Sheltered inlets off } \\
\text { Chesapeake, USA }\end{array}$ & Byme et al. (1981) & med. to fine sand & $2 \pm 1$ \\
\hline $\begin{array}{l}\text { Sheltered inlets, } \\
\text { Queensld., Australia }\end{array}$ & $\begin{array}{l}\text { Riedel \& Gourlay } \\
\text { (1981) }\end{array}$ & fine sand & $2.5 \pm 0.5$ \\
\hline $\begin{array}{l}\text { Western Scheldt, } \\
\text { The Netherlands }\end{array}$ & $\begin{array}{l}\text { de Jong \& Gerritsen } \\
\text { (1985) }\end{array}$ & fine sand & $2.5 \pm 0.5$ \\
\hline
\end{tabular}


Table 3.4. Data for tidal channels and sheltered inlets.

Location

\# of $\quad R_{s p} \quad A \sim Q^{\alpha} \quad A h^{1 / 6} \sim Q^{\beta}$

\begin{tabular}{|c|c|c|c|c|c|}
\hline & Sect & (m) & $\alpha$ & $\beta$ & s.e. \\
\hline Wrecked Recorder Crk., Va., USA & 6 & 1.0 & 1.04 & 1.08 & 0.08 \\
\hline Creek off San Francisco Bay, USA & 10 & 2.6 & 0.91 & 0.97 & 0.07 \\
\hline Delaware Bay, USA & 5 & 1.6 & 0.92 & 0.92 & 0.17 \\
\hline Barnstable*, Massachusetts, USA & 6 & 3.4 & 0.91 & 0.94 & n.a. \\
\hline Alsea, Oregon, USA & 7 & 2.5 & 0.65 & 0.68 & 0.11 \\
\hline Siletz, Oregon, USA & 7 & 2.5 & 0.80 & 0.81 & 0.20 \\
\hline Yaquina, Oregon, USA & 8 & 2.5 & 0.83 & 0.86 & 0.04 \\
\hline Ord, West. Aust., Australia & 3 & 5.9 & 0.97 & 1.02 & 0.04 \\
\hline Thames, England, UK & 10 & 5.2 & 0.94 & 1.00 & 0.06 \\
\hline Creek off Potomac, USA & 6 & 1.0 & 0.92 & 0.96 & 0.01 \\
\hline Forth, Scotland, UK & 12 & 4.9 & 0.98 & 1.02 & 0.06 \\
\hline Savannah, Georgia, USA & 7 & 2.5 & 1.34 & 1.41 & 0.20 \\
\hline Sheltered inlets off Chesapeake, USA & 12 & $.4-.5$ & 0.93 & 1.00 & 0.13 \\
\hline Sheltered inlets, Queensld., Australia & 3 & 2 & 1.12 & 1.22 & 0.03 \\
\hline Western Scheldt, The Netherlands & 9 & 4.4 & 0.97 & 1.02 & 0.02 \\
\hline Tamar, England, UK & 3 & 4.7 & 1.07 & 1.08 & 0.05 \\
\hline Usk, Wales, UK & 24 & 12 & 1.05 & 1.14 & 0.05 \\
\hline Ems, The Netherlands & 8 & 2.6 & 1.04 & 1.08 & 0.03 \\
\hline
\end{tabular}

Mean along-channel $\alpha$ or $\beta$ :

$0.97 \quad 1.01 \pm 0.04$

*Data for individual sections not available 
Figure captions for Chapter 3

Figure 3.1. Observations of cross-sectional area as a function of peak spring discharge at 140 sections from 17 separate tidal channels or shelter inlet systems. Data sources are given in Table 3.1.

Figure 3.2. Observations of the cross-sectional parameter $A h_{R}^{1 / 6}$ as a function of peak spring discharge, the total critical shear stress for non-cohesive sediments, and other "independent" variables, superimposed on the $1: 1$ line given by (3.2.6). Sections with bottom sediments that are known to be non-cohesive are indicated by circles.

Figure 3.3. Observations of the cross-sectional parameter $A h_{R}{ }^{1 / 6}$ as a function of peak spring discharge, the critical erosion shear stress for cohesive sediments, and other "independent" variables, superimposed on the 1:1 line given by (3.2.8). Sections with bottom sediments that are known to be cohesive are indicated by circles.

Figure 3.4. Observations of the cross-sectional parameter $A h_{R}{ }^{1 / 6}$ divided by peak spring discharge, total critical shear stress and other "independent" variables, averaged for each tidal system, and plotted as a function of spring tidal range. Also shown is the leastsquares $\log$-log regression given by (3.3.1). Sections with bottom sediments that are known to be non-cohesive are indicated by circles.

Figure 3.5. Observations of the cross-sectional parameter $A h_{R}^{1 / 6}$ as a function of peak spring discharge, the stability shear stress predicted by (3.3.1), and other "independent" variables, superimposed on the $1: 1$ line given by (3.3.2). Sections with bottom sediments that are known to be cohesive are indicated by circles.

Figure 3.6. Observations along the Thames River of the cross-sectional parameter $A h_{R}{ }^{1 / 6}$ as a function of peak spring discharge, the stability shear stress predicted by (3.3.1), and other "independent" variables, superimposed on the 1:1 line given by (3.3.2). Also shown are least-squares log-log regressions for the three most landward and seven most seaward cross-sections.

Figure 3.7. Representations of idealized equilibrium tidal embayments. Plan views of embayments with intertidal storage areas following (a) a power-law and (b) an exponential relation. A schematic cross-section applicable to either type system is shown in (c). 
Figure 3.8. Map of Wrecked Recorder Creek based on information from Myrick and Leopold (1963). Intertidal storage areas are divided into segments over which timeaveraged embayment width at spring tide is calculated.

Figure 3.9. Map of Ord River based on information from Wright et al. (1973). Intertidal storage areas are divided into segments over which time-averaged embayment width at spring tide is calculated.

Figure 3.10. Least-squares approximations of observed along-channel properties to idealized exponential and/or power-law relations for the Ord River and Wrecked Recorder Creek. (a) Tidal amplitude along the Ord River. (b) Time-averaged spring embayment width along the Ord River and Wrecked Recorder Creek.

Figure 3.11. Comparison of tidal discharge observed along the Ord River and Wrecked Recorder Creek to that predicted by (3.4.6) - (3.4.7).

Figure 3.12. Comparison of the cross-sectional parameter $A h_{R}^{1 / 6}$ observed along the Ord River and Wrecked Recorder Creek to that predicted by (3.4.8) - (3.4.9). 


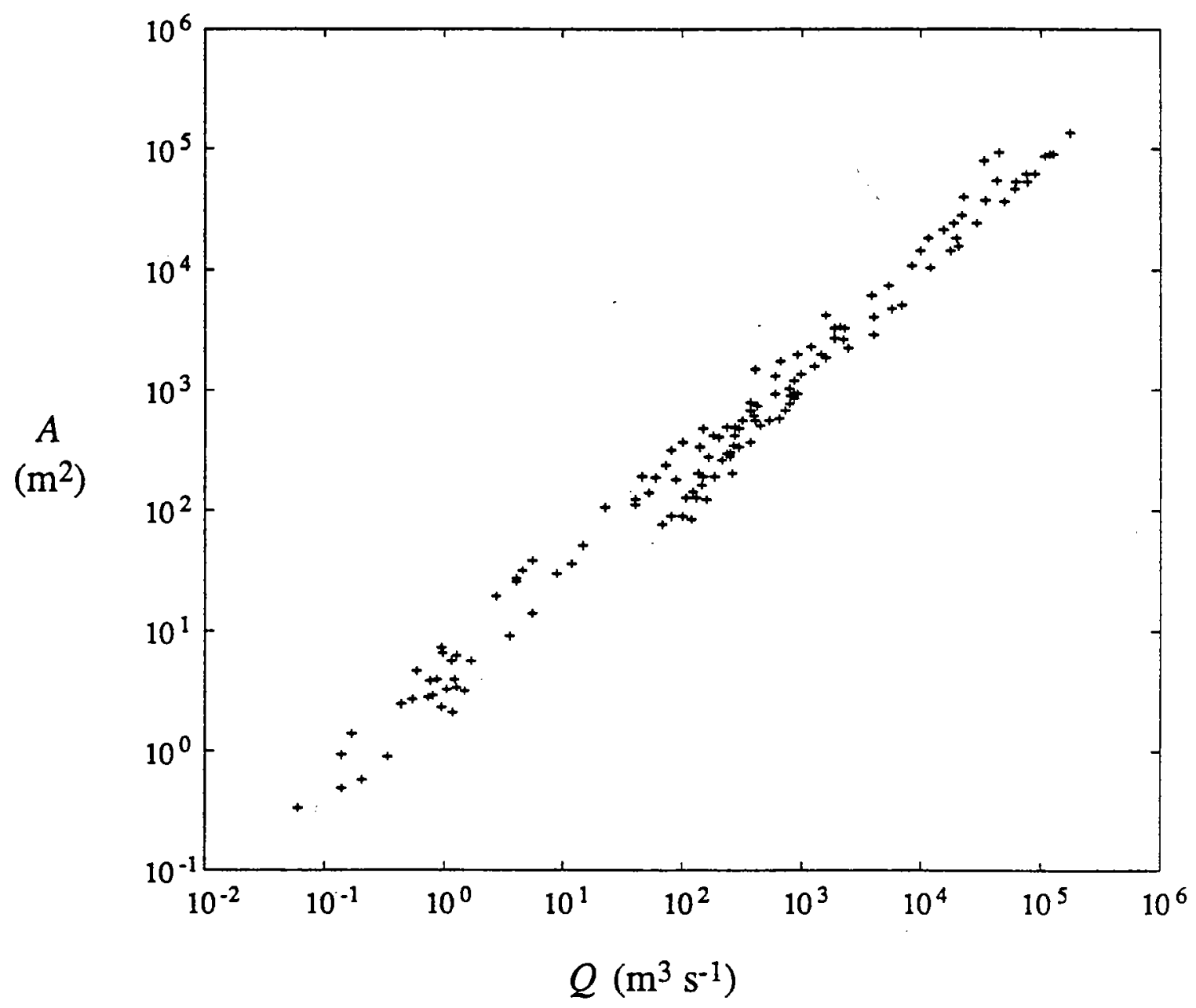

+ cross-sections from Table 3.1

Figure 3.1 


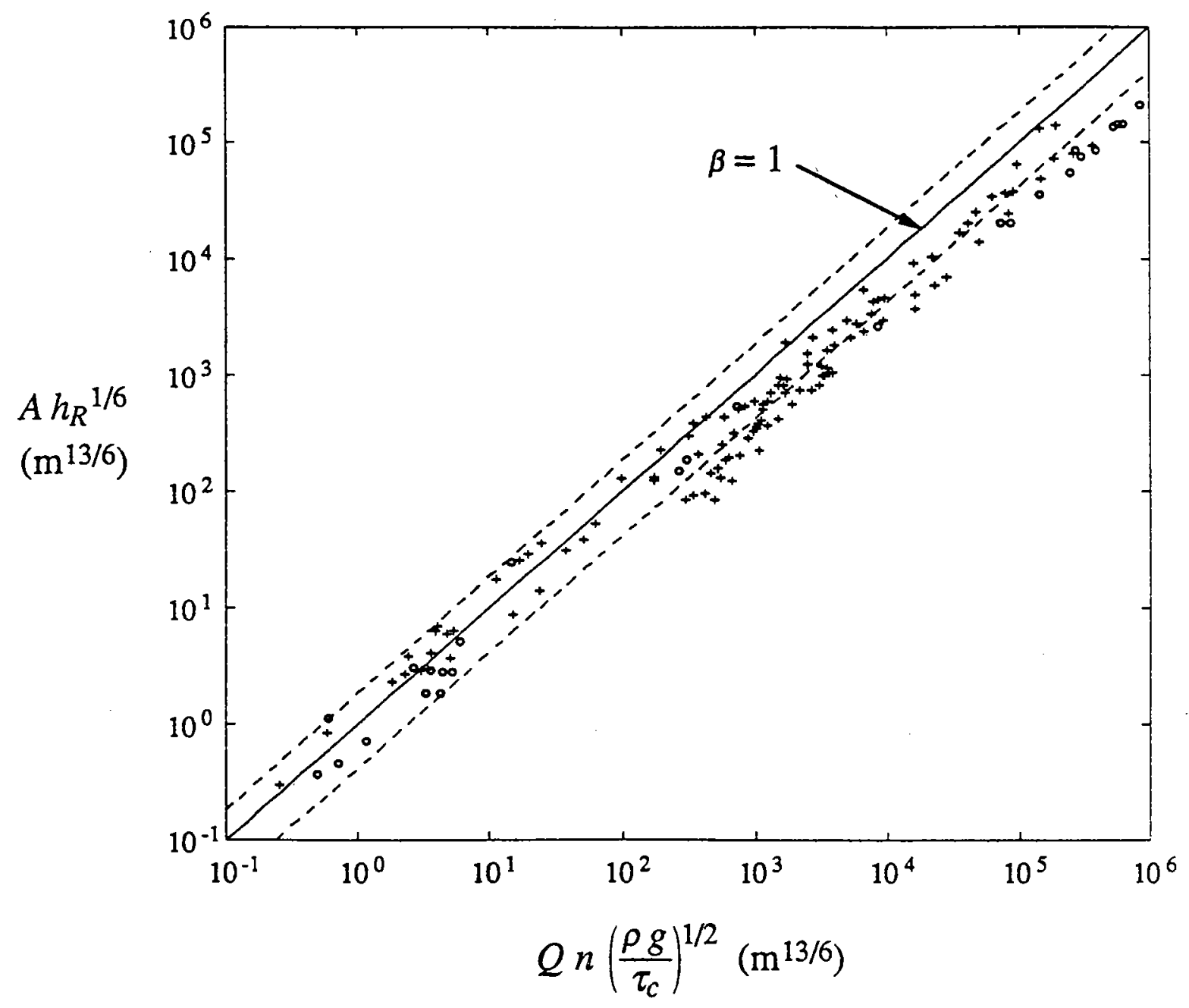

- sections with sediments known to be non-cohesive

- other cross-sections from Table 3.1

Eq. (3.2.6)

error bounds

Figure 3.2 


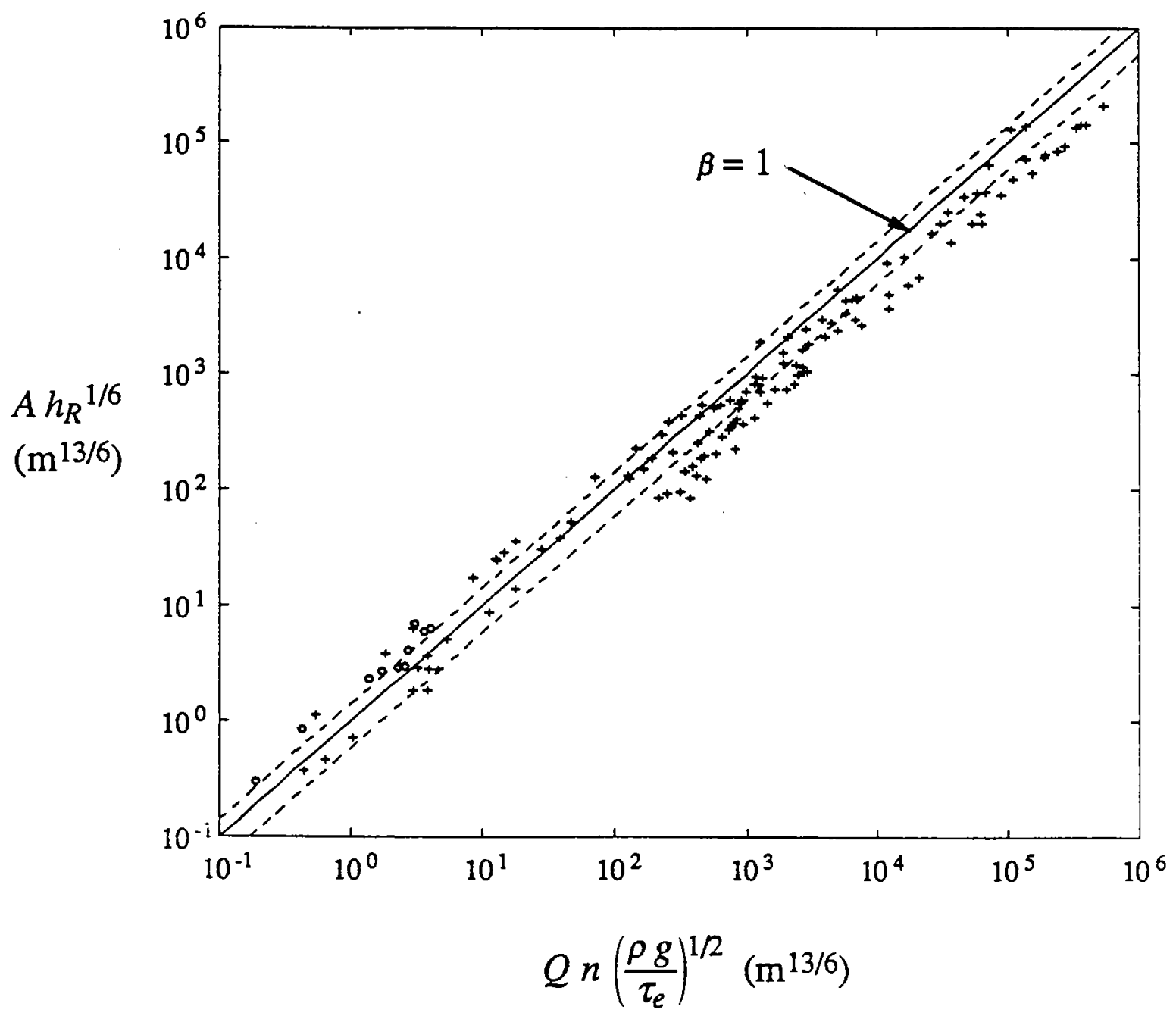

- sections with sediments known to be cohesive

+ other cross-sections from Table 3.1

Eq. (3.2.8)

error bounds

Figure 3.3 


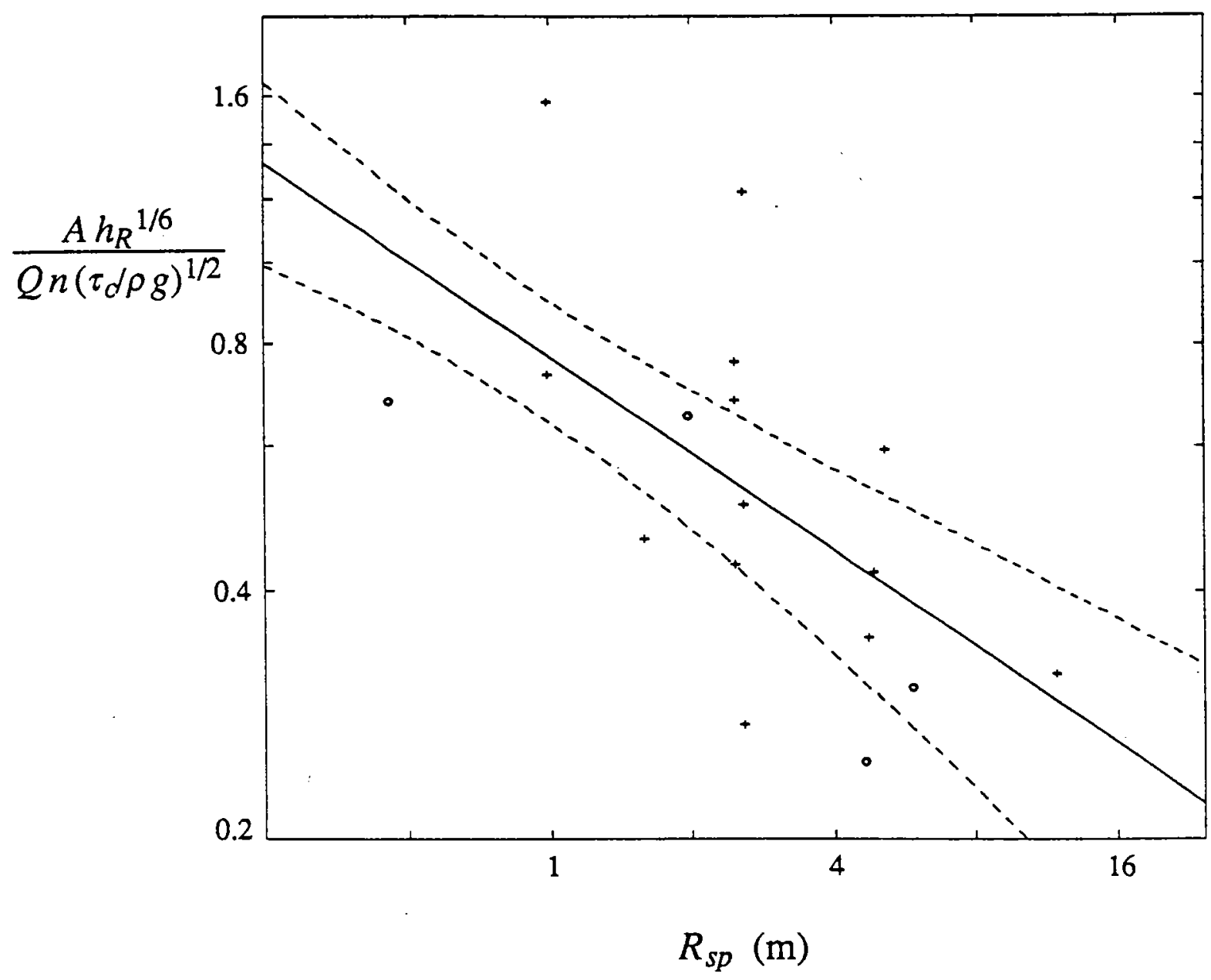

- systems with sediments known to be non-cohesive

+ mean for each other system in Table 3.1

best fit of Eq. (3.3.1)

-...-...- standard errors

Figure 3.4 


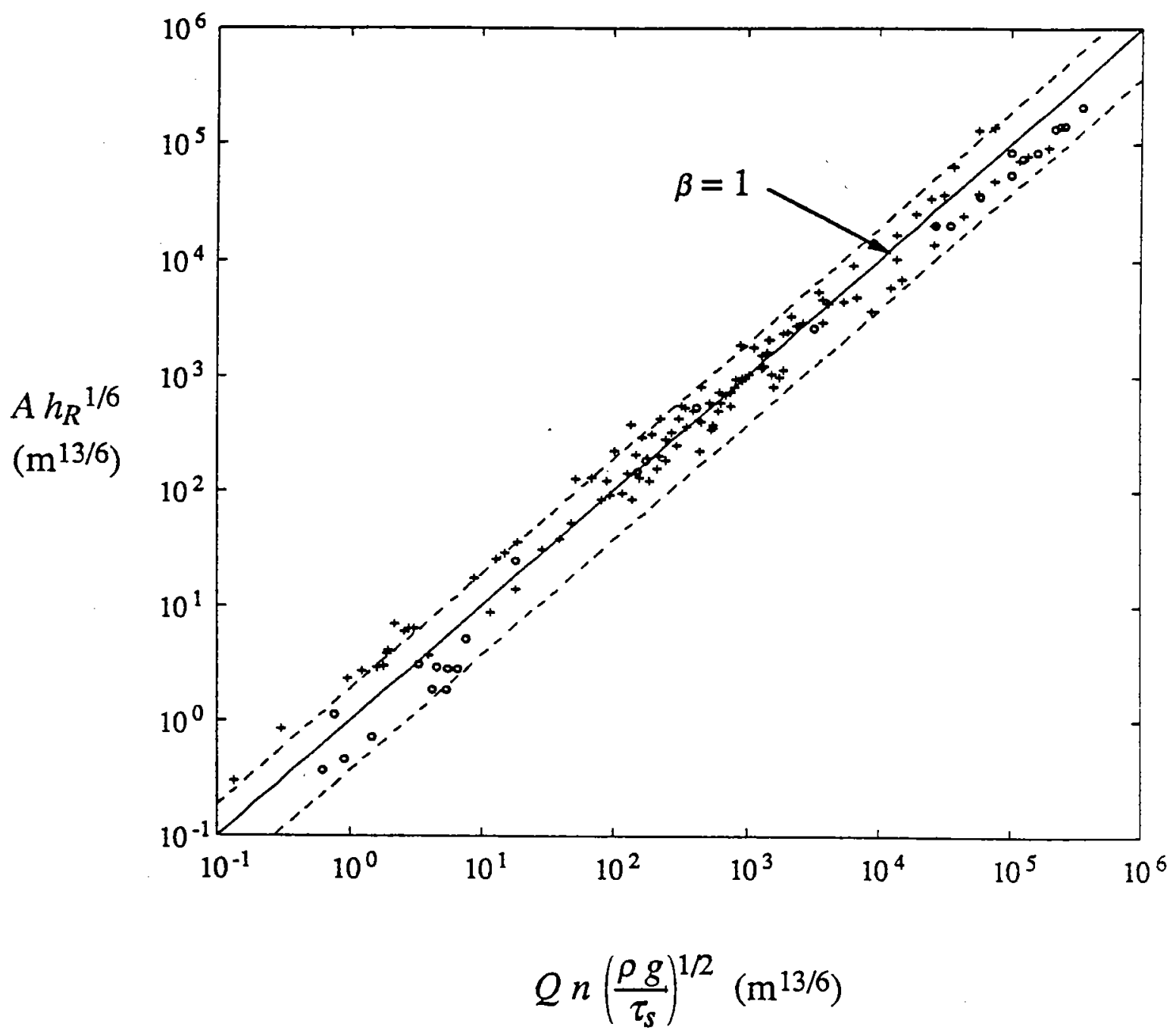

- sections with sediments known to be non-cohesive

- other cross-sections from Table 3.1

Eq. (3.3.2)

error bounds

Figure 3.5 


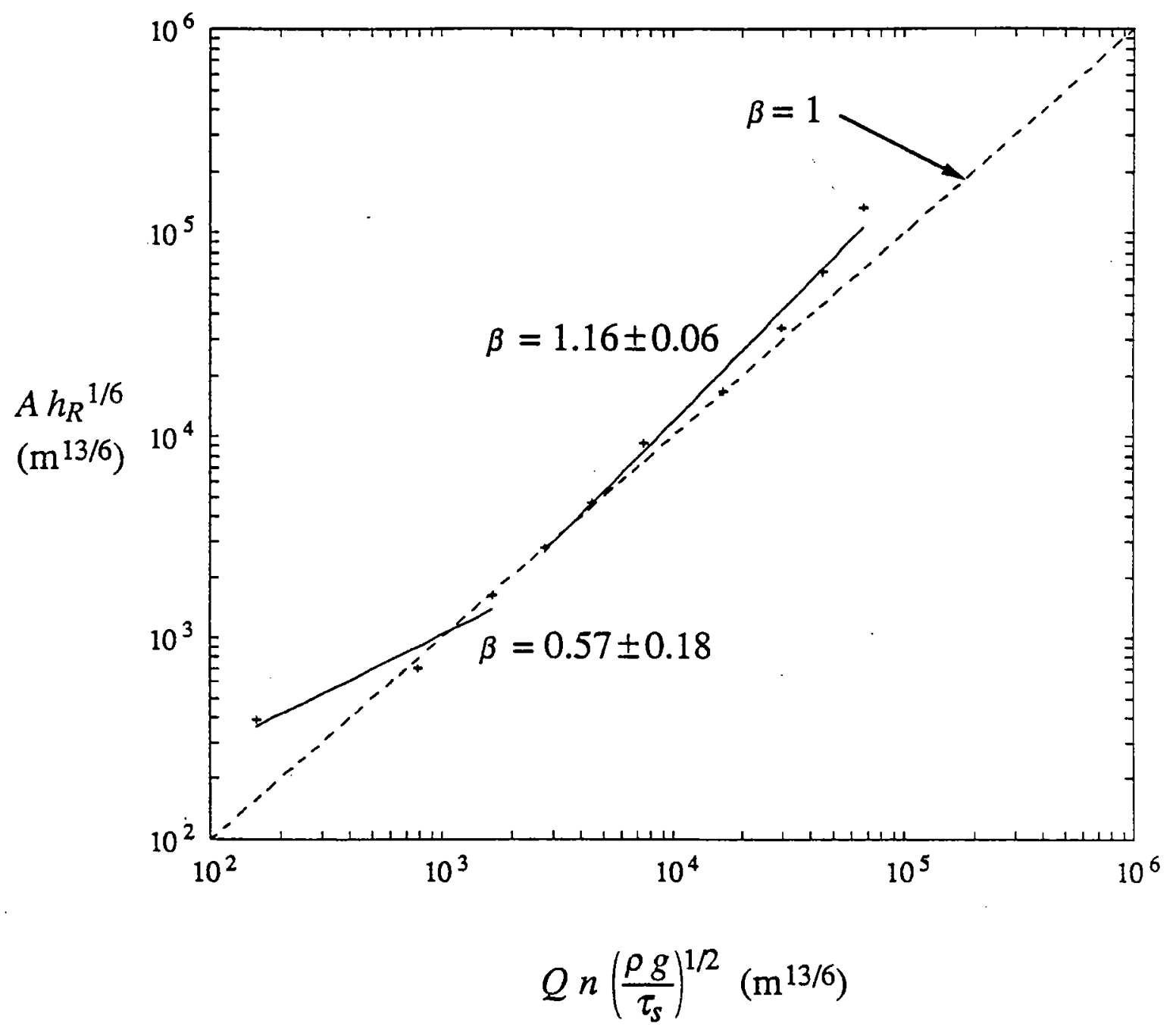

- Thames cross-sections

best fit curves

Eq. (3.3.2)

Figure 3.6 

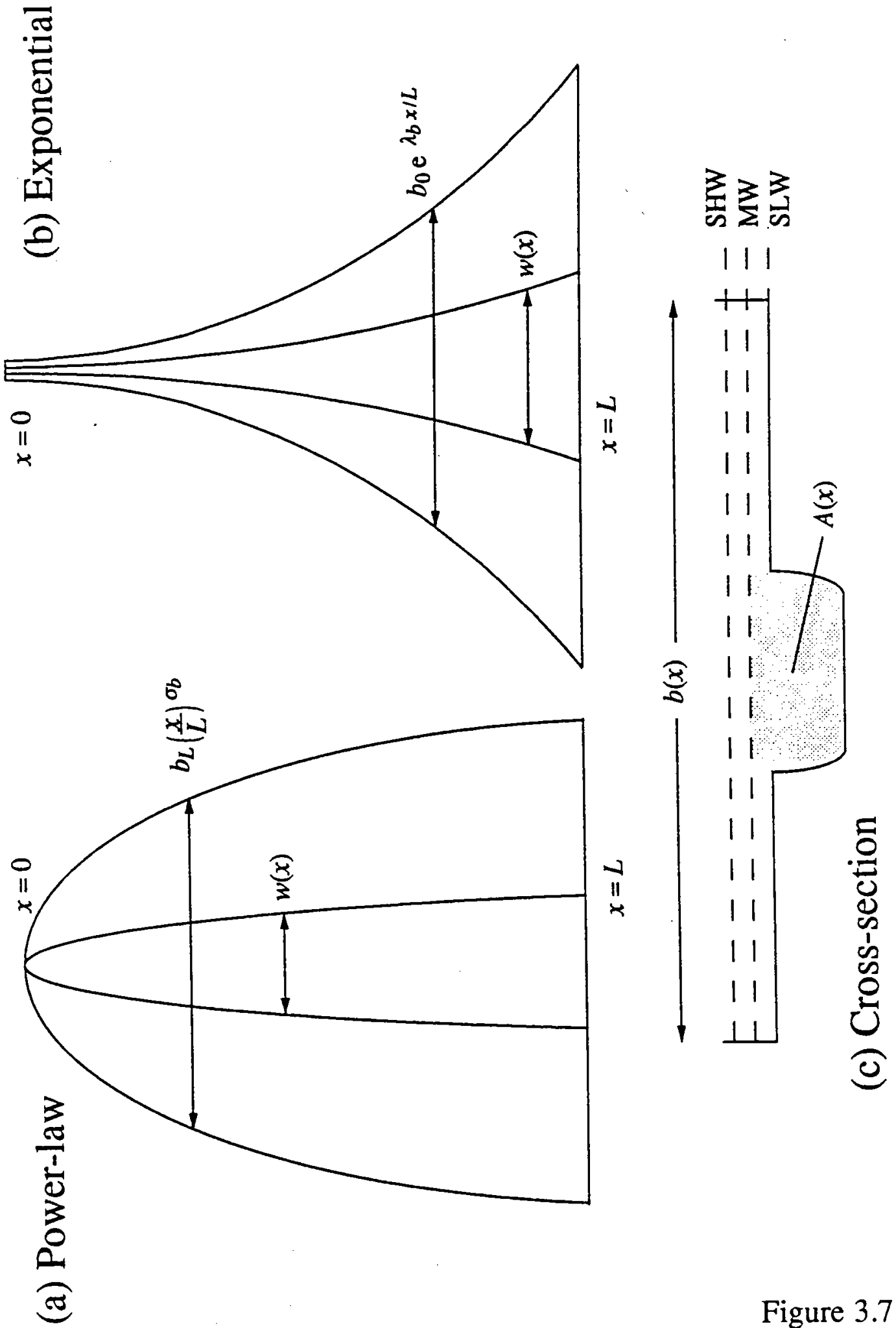

Figure 3.7 
Wrecked Recorder Creek, Virginia

(Myrick and Leopold 1963)

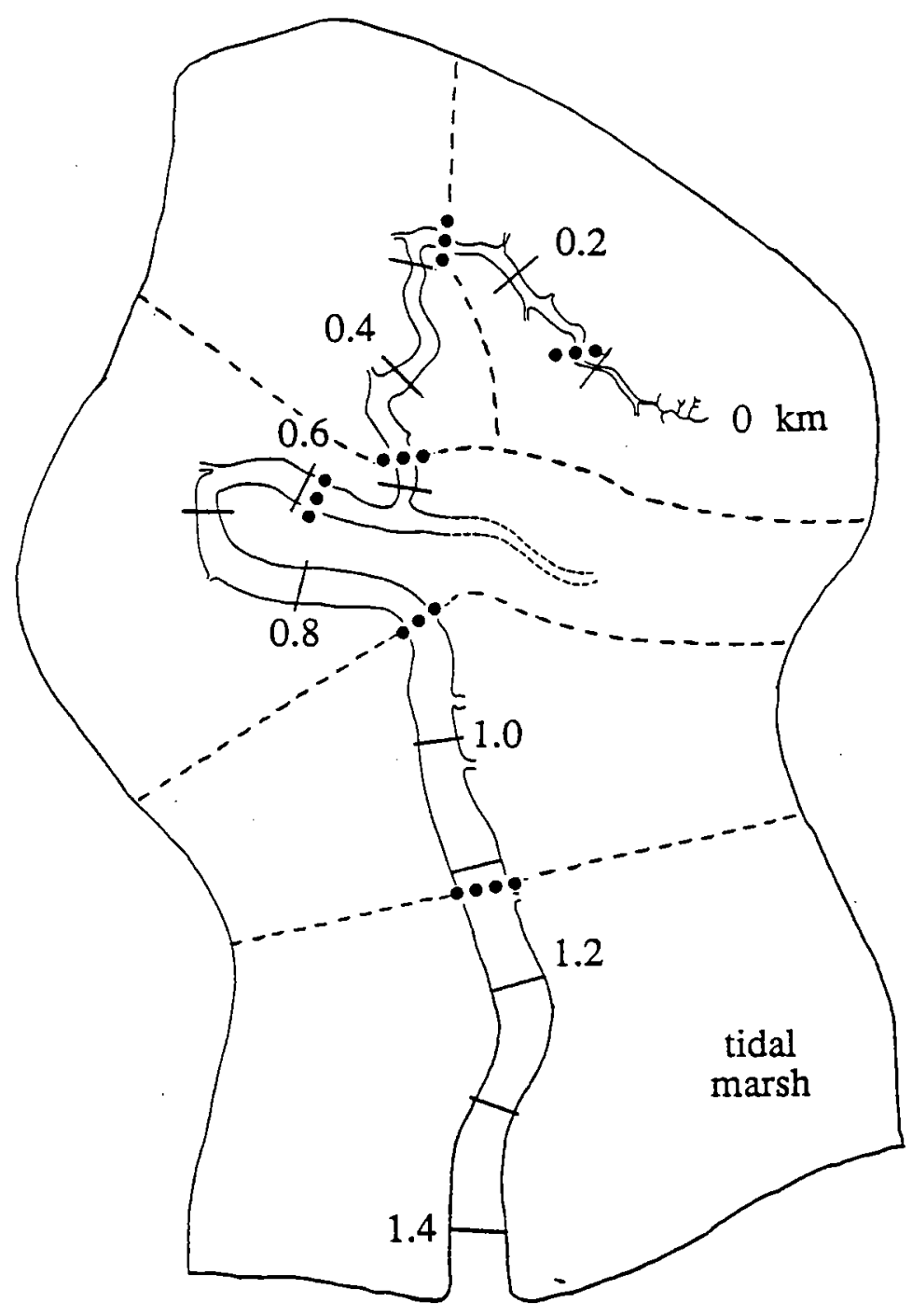

storage segment boundary

.... section in Table 3.1

Figure 3.8 


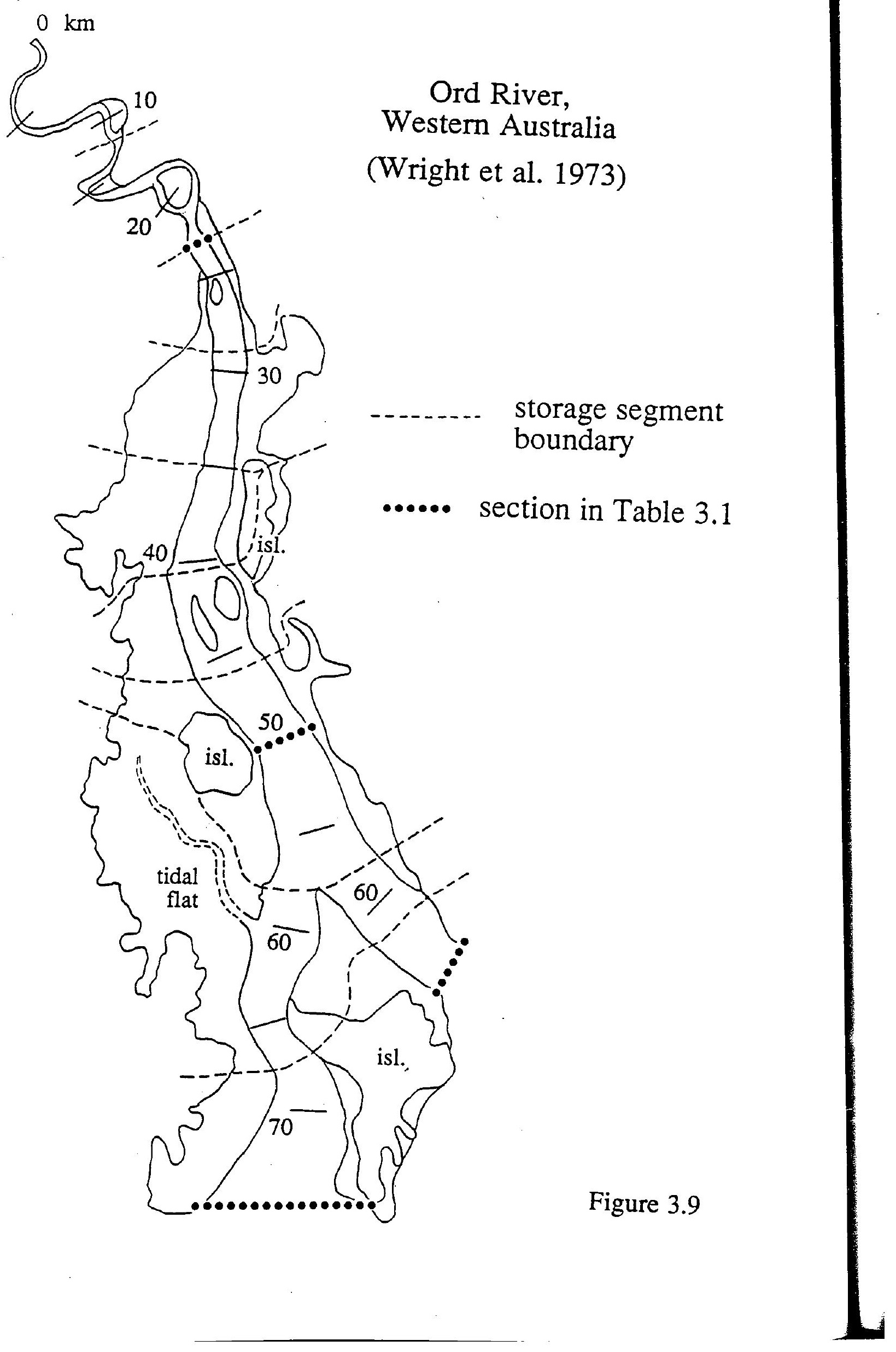



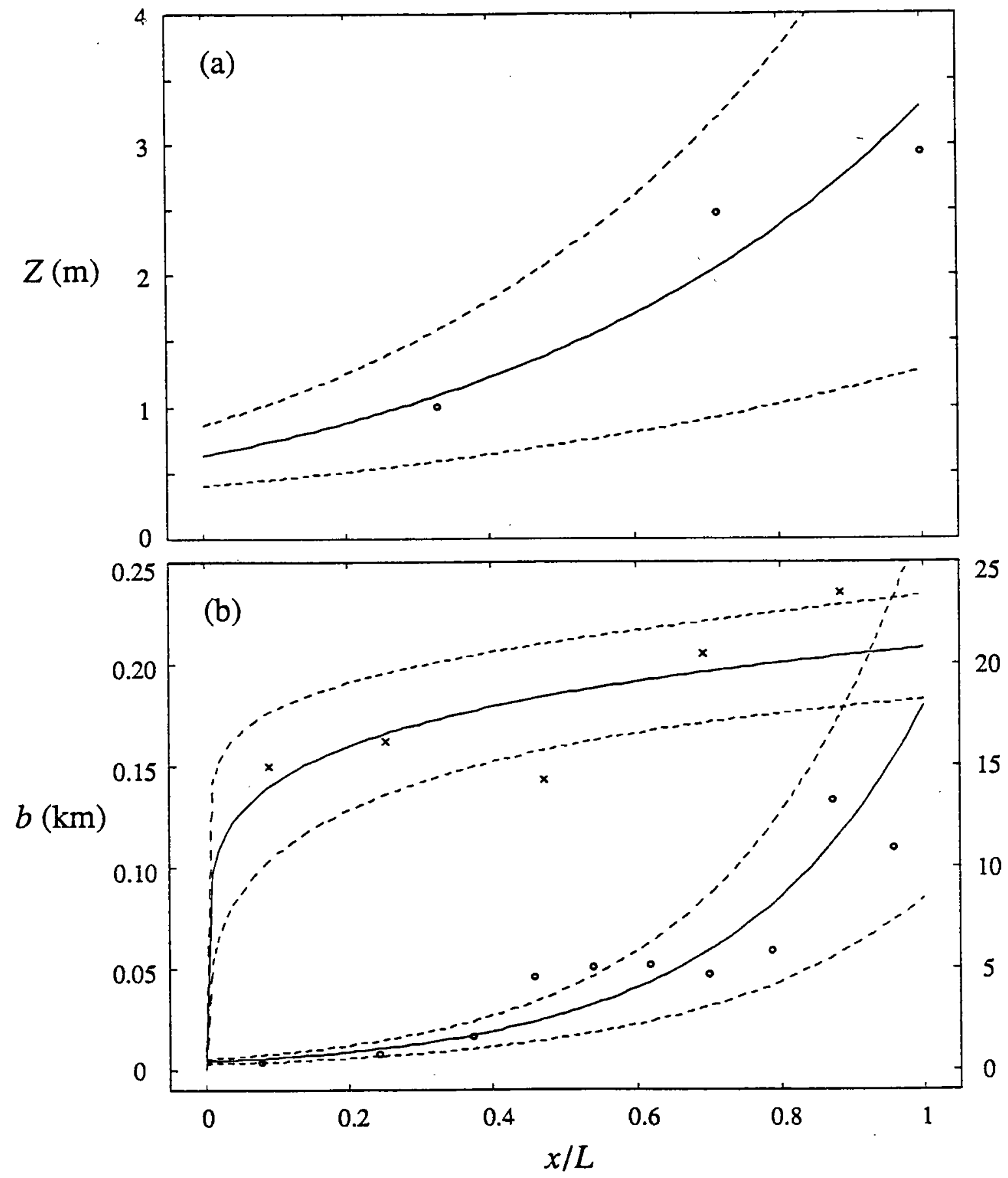

- Ord (Wright et al. 1973) $\quad$ × Wrecked Recorder Creek (Myrick \& Leopold, 1963)

\footnotetext{
— best fits to Eqs. (3.4.6) - (3.4.7)

.......... standard errors
}

Figure 3.10 


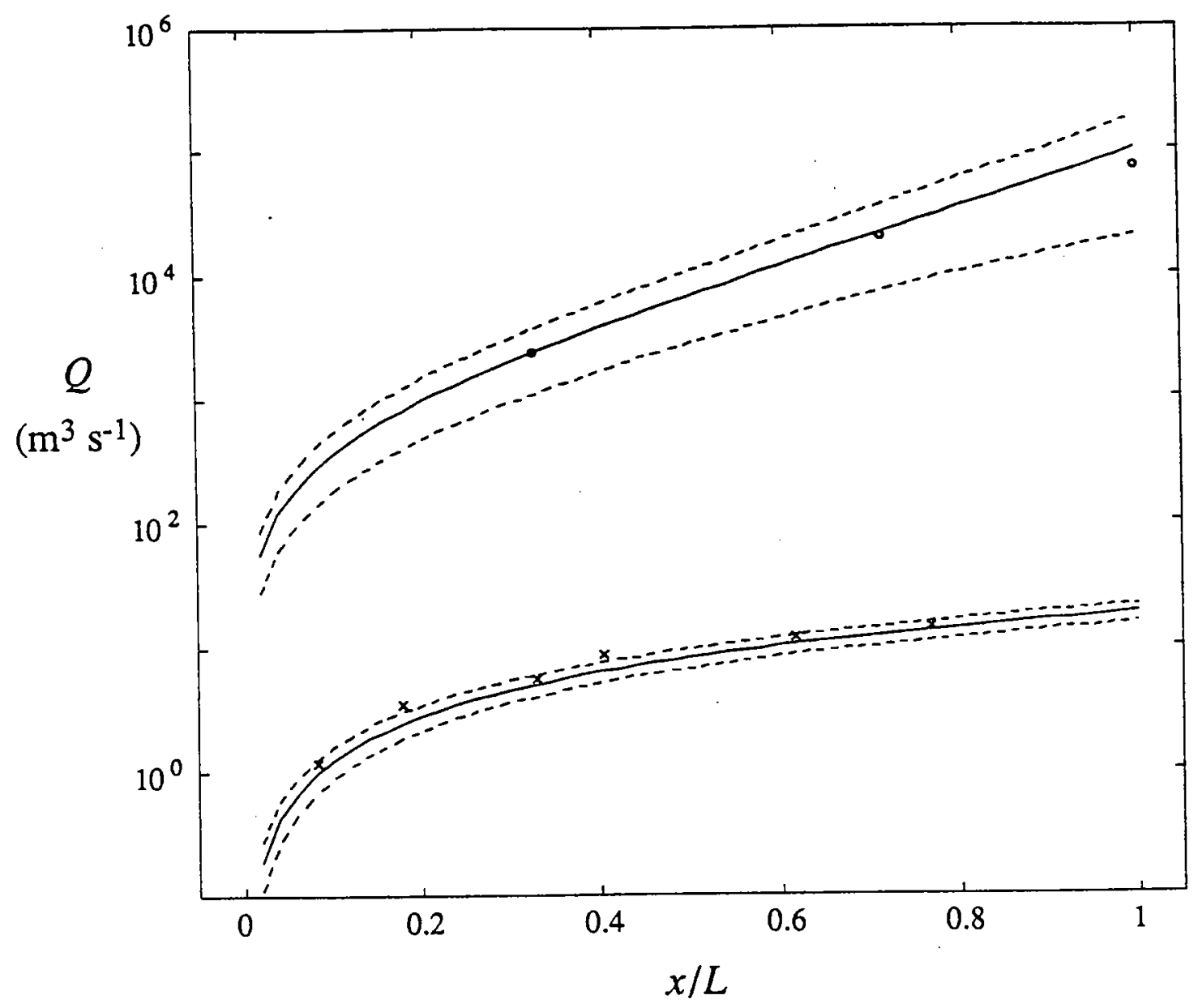

- Ord (Wright et al. 1973) $\quad$ × Wrecked Recorder Creek (Myrick \& Leopold, 1963)

\footnotetext{
Eqs. (3.4.6) - (3.4.7)

.......... standard errors
}

Figure 3.11 


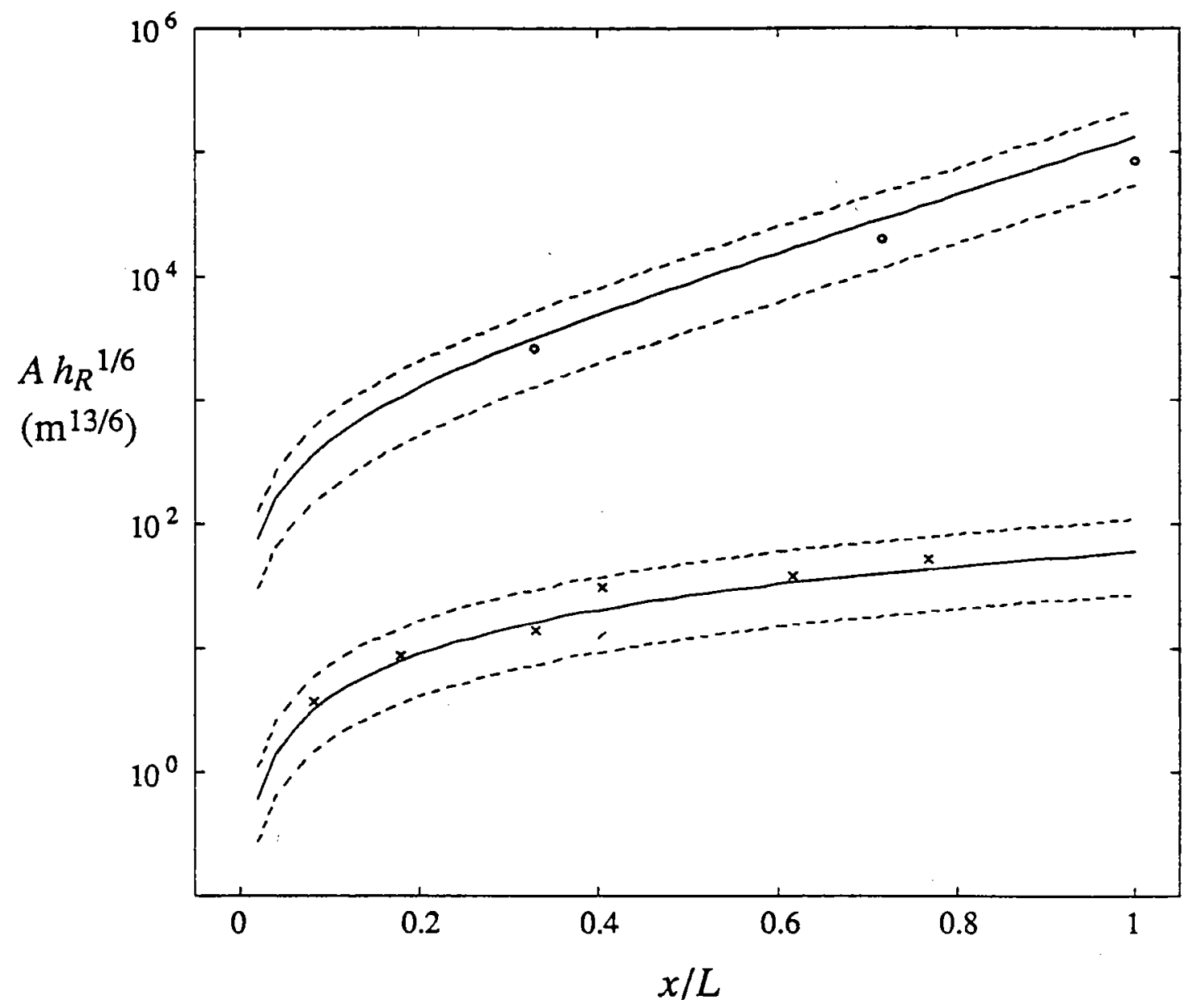

- Ord (Wright et al. 1973) × Wrecked Recorder Creek (Myrick \& Leopold, 1963)

$\longrightarrow$ Eqs. (3.4.8) - (3.4.9)

.....-..-. error bounds

Figure 3.12 


\section{Chapter 4:}

Tidal Propagation in Strongly Convergent Channels Near Morphologic Equilibrium 


\section{Abstract to Chapter 4}

Strongly convergent channels near morphologic equilibrium include such widely studied tidal estuaries as the Delaware in the U.S. and the Thames and the Tamar in the U.K. In this paper a solution is derived for tidal propagation in these channels which diverges from classical views of co-oscillating tides. At each step of the derivation, theoretical predictions are compared with observations from the Delaware, Thames and Tamar. A scaling of the governing equations appropriate to these channels indicates that at first order, gradients in cross-sectional area dominate velocity gradients in the continuity equation and the friction term dominates acceleration in the momentum equation. Finite amplitude effects, phase-generated velocity gradients and local acceleration all enter the equations at second-order.

The first-order governing equation for elevation is a first-order wave equation, in contrast to the classical second-order equation which results from low friction and a prismatic channel. The first-order solutions are constant amplitude, forward-propagating wave forms with velocity leading elevation by $90^{\circ}$, unlike the conventional view of cooscillating tides. Velocity and elevation have the same phase relation as a standing wave, yet they are individually progressive. Also, the solutions are independent of channel length -- in contrast to the length-sensitive resonance of classical co-oscillation.

The second-order governing equation is also a first-order wave equation, and solutions at the dominant frequency propagate at the first-order wave speed. At secondorder, however, the phase lead of velocity decreases as channel convergence decreases, and amplitudes are modulated by $\mathrm{e}^{\mu k x}$, where $k$ is the first-order wave number. The parameter $\mu$ incorporates the partially cancelling effects of local acceleration and limited along-channel convergence. Because $\mu$ determines changes in velocity magnitude, $\mu \approx 0$ along channels which are near morphologic equilibrium. The second-order solution demonstrates that undulations in amplitude and phase along these channels are due primarily to changes in the rate of channel convergence rather than interactions between incident and reflected waves as predicted by classical co-oscillation.

Compact solutions are also provided for the amplitude and relative phase of the second harmonics of elevation and velocity. The harmonics are scaled by $\gamma=a / \bar{h}-\Delta b / \bar{h}$, where $a$ is tidal amplitude, $\bar{h}$ is channel depth, $\bar{b}$ is system width and $\Delta b$ is the amplitude of tidal variation in width. If $\gamma>0$, the wave crest propagates faster than the trough, and rising elevations are of shorter duration. If $\gamma<0$, the opposite holds and falling elevations are shorter. Unlike elevation, velocity asymmetry is predicted to reverse a short distance into the channel. For $\gamma>0$, ebb currents dominate at the mouth, whereas flood currents dominate within the channel. For $\gamma<0$, the opposite pattern is predicted. 
List of symbols in Chapter 4

a amplitude of $\zeta$

$A$ cross-sectional area of channel

$\bar{A} \quad$ time-average of $A$

$A_{0} \quad \bar{A}$ at seaward end of channel

$b \quad$ total system width

$\bar{b}$ time-average of $b$

$b_{0} \quad \bar{b}$ at seaward end of channel

C propagation speed of tidal wave-form

$c_{d} \quad$ drag coefficient

$c_{g} \quad$ frictionless shallow water wave speed

$F \quad$ friction term in momentum equation

$g$ acceleration of gravity

$h$ cross-sectionally averaged channel depth

$\bar{h}$ time-average of $h$

$h_{R} \quad$ hydraulic radius

$-j \quad$ signifies value of variable $\_$for $j$ th channel segment

$k \quad$ tidal wave number

$L \quad$ total length of tidal channel

$L_{a} \quad$ e-folding length of along-channel variations in $a$

$L_{A} \quad$ e-folding length of along-channel variations in $\bar{A}$

$L_{b} \quad$ e-folding length of along-channel variations in $\vec{b}$

$L_{g} \quad$ frictionless shallow water wavelength

$L_{T} \quad$ length of tidal wave form

$L_{U} \quad$ e-folding length of along-channel variations in $U$

$m \quad$ signifies $m$ th harmonic component

$r \quad$ linearized friction coefficient

$t$ time

$u \quad$ cross-sectionally averaged velocity

$U$ amplitude of $u$

$U_{0} \quad U$ at seaward end of channel

$V_{m} \quad m$ th harmonic of velocity in $t$-domain

$w \quad$ channel width

$x \quad$ along-channel co-ordinate

$x_{0} \quad$ representative location along channel 
List of symbols in Chapter 4 (continued)

$x_{b} \quad$ location of tidal barrier

$Z_{m} \quad$ real amplitude of $\zeta_{m} / a$ at $x=0$

$\alpha_{m} \quad$ complex amplitude of $\zeta_{m}$ at $x=0$

$\gamma \quad \varepsilon_{h}-\varepsilon_{b}$

$\Delta b \quad$ amplitude of tidal variation in $b$

$\varepsilon \quad$ signifies second-order term

$\varepsilon_{a} \quad L_{A} / L_{a}$

$\varepsilon_{b} \quad \Delta b / \bar{h}$

$\varepsilon_{h} \quad a / \bar{h}$

$\varepsilon_{T} \quad 2 \pi L_{A} / L_{T}$

$\varepsilon_{U} \quad L_{A} / L_{U}$

$\varepsilon_{\omega} \quad \omega / r$

$\zeta \quad$ tidal elevation

$\zeta_{m} \quad m$ th harmonic of elevation in $\tau$-domain

$\eta_{m} \quad m$ th harmonic of elevation in $t$-domain

$\theta \quad$ tidal phase

$\theta_{m} \quad$ phase angle of $\zeta_{m}$

$\varphi_{V m} \quad$ phase angle of $V_{m}$

$\varphi_{\eta m} \quad$ phase angle of $\eta_{m}$

$\lambda \quad L / L_{A}-L / L_{b}$

$\mu \quad \varepsilon_{\omega}-\varepsilon_{T}$

$\xi \quad x$-dependent portion of $\zeta$

$\tau$ transformed time variable

$\omega \quad$ tidal frequency 


\subsection{Introduction}

In his treatise on tidal hydraulics, Pillsbury (1939) defines an "ideal estuary" as one where "simple harmonic fluctuation of the tide at the entrance will produce throughout the channel primary tides of constant range and primary currents of uniform strength" (p. 228). In this paper a new solution describes tidal propagation in just such a channel. The channel characteristics which lead to this solution are strong along-channel convergence of crosssectional area together with a distribution of velocity amplitude indicative of morphologic equilibrium. This channel type is common and includes such widely-studied tidal estuaries as the Delaware in the U.S. and the Thames and the Tamar in the U.K. The governing equation and solution appropriate to these channels diverge from classical views of damped tidal co-oscillation. It is thus useful to review briefly the classical approach to the propagation of tides in channels.

\subsubsection{Classical tidal co-oscillation}

The classic approach to tides in channels (e.g., Ippen 1966) derives basic wave patterns of interest to oceanographers with little or no consideration of friction or channel convergence. In a frictionless, prismatic channel of rectangular cross-section, the 1-D linearized governing equation for elevation $(\zeta)$ reduces to the familiar second-order wave equation:

$$
\frac{\partial^{2} \zeta}{\partial t^{2}}=c_{g}^{2} \frac{\partial^{2} \zeta}{\partial x^{2}}
$$

where $t$ is time, $x$ is distance, and $c_{g}=(g h)^{1 / 2}$.

For a channel closed at one end, (4.1.1) produces a standing wave solution, characterized by incident and reflected waves of equal amplitude. The incident and reflected waves interact to form nodes and anti-nodes which are a function only of the length and depth of the channel. If the channel is exactly one-quarter wave in length, then the incident and reflected waves cancel entirely at the mouth, and infinite resonance occurs within the channel. In his review of tidal dynamics in estuaries, Ippen (1966) provided solutions to (4.1.1) for channels closed at one end, of infinite length, and forced at both ends. Ippen then used Green's Law to examine tides in channels of gradually varying cross-section, but again limited his discussion to the frictionless case. 
The last solution presented by Ippen (1966) is once more for a prismatic, rectangular channel, but for the more "realistic" case of a damped co-oscillating tide which includes the effects of friction. Inclusion of linear friction transforms (4.1.1) into a damped second-order wave equation:

$$
\frac{\partial^{2} \zeta}{\partial t^{2}}+r \frac{\partial \zeta}{\partial t}=c_{g}^{2} \frac{\partial^{2} \zeta}{\partial x^{2}},
$$

where $r$ is a constant friction factor (Ippen 1966). In a channel closed at one end, the solution to (4.1.2) consists of exponentially-modified incident and reflected waves which are of equal amplitude at the landward reflection point. The phase speeds of the incident and reflected waves are equal to each other and, for weak friction, are only slightly less than $c_{g}$. For large $r$, the second term in (4.1.2) dominates the first, and (4.1.2) reduces to a time-varying diffusion equation. The frictionally-dominated asymptote is addressed in detail in Chapter 2 of this thesis.

For short channels (having lengths less than a quarter tidal wavelength), solutions for the amplitude of $\zeta$ derived from either (4.1.1) or (4.1.2) can be quite similar to observations. Furthermore, tidal phase in real channels is often observed to propagate at speeds close to $(g h)^{1 / 2}$. Thus (4.1.1) - (4.1.2), along with $c_{g}=(g h)^{1 / 2}$, are commonly viewed as adequate explanations for most aspects of first-order tidal propagation in natural channels. However, more subtle but important constraints on tidal phase and especially on the solution for cross-sectionally averaged velocity $(u)$ reveal the shortcomings of (4.1.1) (4.1.2) when applied to some real tidal systems.

Tidal phase in many tidal channels is observed to increase linearly landward. However neither (4.1.1) nor (4.1.2) can produce a linear increase in phase along a channel of finite length. In a finite channel, (4.1.1) produces a phase that is everywhere equal, whereas (4.1.2) produces a phase lag that varies non-linearly along-channel. Although observed phase may indeed have a speed near $c_{g}$, neither (4.1.1) nor (4.1.2) actually predicts that it should. $c_{g}$ is the predicted phase speed of the individual forward and backward propagating waves in (4.1.1) - (4.1.2), not the predicted speed for the combination which makes up the observed wave-form.

Along some tidal channels the relative phase of $u$ to $\zeta$ is observed to be nearly constant at $\sim 90^{\circ}$ (Hunt 1964; Wright et al. 1973). Although this is the phase relation for finite channels given by (4.1.1), (4.1.2) predicts that the phase of $u$ to $\zeta$ should be $90^{\circ}$ only 
at the landward reflection point and should decrease monotonically toward the channel mouth. Finally, the scaling inherent in (4.1.1) - (4.1.2) leads to unrealistic values for the amplitude of tidal velocity. In a short prismatic channel, conservation of mass requires $u$ to be proportional to distance from the reflection point, whether applying (4.1.1) or (4.1.2). As shown in Chapter 3 of this thesis, however, the amplitude of $u$ is nearly constant along the length of real tidal channels which are near morphologic equilibrium. The only way to predict realistic first-order solutions for channels near morphologic equilibrium is to include both friction and along-channel variation in cross-sectional area.

\subsubsection{Previous solutions for convergent tidal channels with friction}

Perroud (1959) re-expressed the linearized 1-D equations of motion for tides in terms of particle displacement and considered channels with (i) linearly varying width and constant depth, (ii) exponentially varying width and constant depth, and (iii) uniform width and linearly varying depth. Solutions for (i) and (iii) were reached via Bessel functions, whereas the solution for (ii) was reached via trigonometric functions. In all three cases, solutions for $\zeta$ were expressed in terms of incident and reflected waves modified by exponentials. The phase speed for the incident and reflected waves was presented as a perturbation around $c_{g}$, and no solutions were provided for $u$. There was no discussion or even graphical representation of the solutions and no comparison to observations. Thus it is not surprising that the work of Perroud (1959) did little to change the common view of tidal co-oscillation.

Hunt (1964) was the first to emphasize the fundamentally different nature of tidal propagation along convergent channels having friction. Hunt solved the linearized 1-D equations for (i) exponentially varying width and constant depth and (ii) linearly varying depth and width varying like $x^{3 / 2}$. Like Perroud (1959), Hunt solved (i) with sinusoids and (ii) via Bessel functions. Hunt pointed out that, unlike solutions for prismatic channels, solutions for convergent channels can produce a progression in tidal phase alongchannel while simultaneously maintaining a relative phase of $u$ to $\zeta$ near $90^{\circ}$. He even suggested that the previous agreements of observed phase speed with $(g h)^{1 / 2}$ were "both fortuitous and misleading" (p. 442). He de-emphasized the importance of incident and reflected waves by expressing his solutions as single, exponentially modified, forward propagating wave-forms. Finally, Hunt showed his analytic solution to be consistent with observations from the Thames. 
Prandle and Rahman (1980) used Bessel functions to derive solutions for channels whose widths and depths obey arbitrary power laws and compared their results to observations from a wide range of tidal systems. Their generalized solutions are a function of only three parameters: one which scales channel convergence, one which indicates position along channel, and one which indicates the strength of friction relative to acceleration. Prandle and Rahman stressed the role of nodal points in determining the nature of the solutions, thus emphasizing similarities between their solutions and classic damped co-oscillation. They did note, however, that tidal amplification was controlled by the geometry of the channel as a whole and not simply by its length and depth.

Jay (1991) used a modified Green's function approach for channels having exponentially varying width and depth, but did not compare analytic results to observations. Jay showed wave behavior at lowest order to be a function of channel convergence and the importance of friction relative to acceleration. Like Hunt (1964), Jay emphasized the role of channel convergence in producing a wave-form which is fundamentally different from classical damped co-oscillation. Jay found that a single incident wave in a channel with strongly convergent geometry may mimic a standing wave by having a relative phase of $u$ to $\zeta$ near $90^{\circ}$ without the presence of a reflected wave. Jay also found that under conditions of "critical convergence" the effects of channel convergence and local acceleration cancel entirely at first-order, leaving an effective balance between friction and pressure gradient.

A likely explanation for the continued emphasis on damped co-oscillation, despite the important contributions of Hunt (1964), Jay (1991) and others, is the complicated form of their solutions. Application of either Hunt or Jay's solution requires repeated transformations and/or substitutions which partly obscure the solution's intuitive impact. Simple interpretation of solutions based on Bessel functions is even more difficult. Unfortunately, the solution and (mis)interpretation of (4.1.2) is still much simpler in practice.

Part of the difficulty in interpreting the results of these previous investigators stems from the large number of first-order terms they have all kept in the equations of motion. All previous investigators of convergent systems have assumed that (i) friction scales no larger than local acceleration in the momentum equation, and (ii) discharge gradients due to channel convergence scale no larger than those due to velocity gradients in the continuity equation. This choice of first-order scaling is inappropriate to the class of channels of 
interest to this study. The above scaling may have been applied in the past to shallow, strongly convergent channels due to a lack of comparison to observations from real tidal channels during the scaling process. Of course near-resonant, convergent tidal systems do exist where acceleration is as or more important than friction. Examples include the Gulf of Maine and the Bristol Channel, both of which were examined by Prandle and Rahman (1980). However these systems are hundreds of kilometers in length and many tens of meters deep.

In the following section a careful scaling of the equations of motion is performed which is appropriate to an arguably more common type of strongly convergent tidal channel, namely those having depths on the order of ten meters or less and which are near morphologic equilibrium. Scaling appropriate to such real tidal channels as the Delaware in the U.S. and the Thames and the Tamar in the U.K. indicate that at first order, gradients in cross-sectional area dominate the continuity equation and friction dominates in the momentum equation. This realization leads to a simpler first-order governing equation which has the form of a first-order wave equation. The solution includes all the major properties which distinguish tidal waves in strongly convergent channels, yet is more amenable to conceptual interpretation. Finite amplitude effects, velocity gradients and local acceleration, which all enter the equations at second order, then lead to systematic, interpretable perturbations on the first-order solution.

\subsection{Scaling of equations}

The cross-sectionally integrated, 1-D equations for a tidal channel with linearly sloping intertidal flats (Figure 4.1) may be expressed as (Speer and Aubrey 1985)

$$
\begin{array}{lc}
\text { continuity: } & b \frac{\partial \zeta}{\partial t}=-\frac{\partial}{\partial x}\{A u\}, \\
\text { momentum: } & \frac{\partial u}{\partial t}+u \frac{\partial u}{\partial x}=-g \frac{\partial \zeta}{\partial x}-F,
\end{array}
$$

where $b(x, t)$ is total system width (including flats), $\zeta(x, t)$ is tidal elevation, $A(x, t)$ is crosssectional area of the channel (excluding flats), $u(x, t)$ is cross-sectionally averaged velocity (confined to the channel), and $F$ represents bottom friction. In addition to the usual assumptions of channelized flow, (4.2.1) - (4.2.2) assume $u=0$ on the flats (Speer and Aubrey 1985). 
The tidal channels considered here are near morphologic equilibrium such that the amplitude of tidal velocity, $U$, varies only weakly in $x$. To simplify the scaling arguments, it is assumed that the amplitude of tidal elevation, $a$, also varies only weakly in $x$ and that the solutions for $\zeta$ and $u$ can be approximated by the following progressive wave forms:

$$
\zeta=a \cos (\omega t-k x), \quad \text { and } \quad u \approx U \cos (\omega t-k x-\theta)
$$

where $\omega$ is the tidal radian frequency, $k=2 \pi / L_{T}$ is the tidal wave number where $L_{T}$ is the observed tidal wavelength, and $\theta$ is the relative phase of tidal velocity.

These assumptions reasonably fit such well-studied tidal estuaries as the Delaware in the United States (Harleman 1966; Parker 1984), and the Thames (Hunt 1964; Prandle 1980), and the Tamar (George 1975; Uncles et al. 1985; Uncles and Stephens 1990) in the United Kingdom. As discussed in Chapter 3, the amplitude of cross-sectionally averaged tidal velocity is nearly constant along the lengths of all three of these estuaries (Figure 4.2). Values for $L_{T}$, determined from the slopes of the linear regressions in Figure $4.2 \mathrm{~b}$, are listed in Table 4.1. Observations shown in Figure 4.2 for the Delaware and the Thames are $\mathrm{M}_{2}$ data from Parker (1984) and Prandle (1980), respectively. Data for the Tamar are spring tide values from George (1975). George (1975) provides spring and neap, but not mean tide data. Only the spring observations are examined here because under spring conditions, the tide is more likely to dominate river flow, and freshwater effects are not a focus of this investigation.

In the following paragraphs, the continuity equation is examined first to determine which terms must be retained at first and second order. Results from continuity are then used similarly to scale the momentum equation. (For reference, the dimensionless quantities which are assumed to be small in this study are summarized in Table 4.2.)

\subsubsection{Scaling of continuity}

For estuaries represented by Figure 4.1, continuity may be expanded as

$$
\bar{b} \frac{\partial \zeta}{\partial t}+\varepsilon_{b} \frac{\zeta}{a} \bar{b} \frac{\partial \zeta}{\partial t}=-\left(\frac{\mathrm{d} \bar{A}}{\mathrm{~d} x}\right) u-\varepsilon_{h} \frac{\zeta}{a}\left(\frac{\mathrm{d} \bar{A}}{\mathrm{~d} x}\right) u-\left(1+\mathrm{O}\left(\varepsilon_{h}\right)\right) \bar{A} \frac{\partial u}{\partial x} .
$$

The first two terms on the right hand side of (4.2.4) arise from the along-channel gradient of cross-sectional area, whereas the last term on the right is due to the along-channel 
gradient in tidal velocity. The small parameters $\varepsilon_{h}$ and $\varepsilon_{b}$ arise from finite amplitude and intertidal slope effects and are defined as:

$$
\varepsilon_{h}=\frac{a}{\bar{h}}, \text { and } \varepsilon_{b}=\frac{\bar{b}-w}{\bar{b}}=\frac{\Delta b}{\bar{b}}
$$

Over-bars indicate time-averages such that $h=\bar{h}+\varepsilon_{h} \zeta, b=\bar{b}+\varepsilon_{b} \zeta$, and $A=\bar{A}+\varepsilon_{h} \zeta w$. $h(x, t)$ is cross-sectionally averaged channel depth, and $w(x)=A / h$ is both the width of the channel and the total embayment width at low tide (see Figure 4.1). Observed values for $\varepsilon_{h}$ and $\varepsilon_{b}$ are given in Table 4.1 .

In order to scale the terms in (4.2.4), estuary width, cross-sectional area, and tidal velocity are assumed to vary as:

$$
\bar{b}=b_{0} \mathrm{e}^{-x / L_{b}}, \bar{A}=A_{0} \mathrm{e}^{-x / L_{A}}, \text { and } U=U_{0} \mathrm{e}^{ \pm x / L U}
$$

where $L_{b}, L_{A}$ and $L_{U}$ are e-folding lengths of along-channel variation, and $x=0$ at the forced end of the channel. Observed $\bar{A}$ and $\bar{b}$ for the Thames are taken directly from previous compilations (Hunt 1964; Parker 1984). Observed $\bar{b}$ for the Thames is determined from U.S.D.M.A. charts 37145 and $37146 . \bar{A}$ and $\bar{b}$ for the Tamar are calculated from channel cross-sections published by Uncles et al. (1985). The division between the momentum transporting channel and the "storage only" flats in the Tamar cross-sections is based on the break in bank slope and corresponds roughly to the neap low water line.

Observed $U$ values for the Delaware and Thames are taken directly from previous compilations (Harleman 1966; Chantler 1974). For the Delaware, Harleman (1966) did not measure $U$ directly, but calculated the cross-sectionally averaged velocity via integration of observed tidal elevations. For the Thames, Chantler (1974) presented values for $U$ as a function of $\bar{A}$. In Figure $4.3 \mathrm{~b}, x$-values were estimated for the Thames by interpolating from the $\bar{A}(x)$ data provided by Hunt (1964). Observed $U$ for the Tamar is calculated from harmonic analyses of time-series of sectionally-averaged spring velocity presented by Uncles et al. (1985).

Figure 4.3 illustrates the fit of observed $\bar{b}, \bar{A}$ and $U$ to exponential curves, and Table 4.1 lists observed values for $L_{b}, L_{A}$ and $L_{A} / L_{U}$. Clearly $U$ does not generally follow a simple exponential curve over the length of an entire estuary. The main purpose here is to illustrate that $L_{b}$ and $L_{A}$ are much less than $L_{U}$ on a system-wide scale. 
For tides and estuaries described by (4.2.3b) and (4.2.6), Eq. (4.2.4) may reexpressed in terms of scales as follows:

$$
\begin{aligned}
& \frac{a \omega L_{A}}{(\bar{A} / \bar{b}) U}\left(1+\varepsilon_{b}\right)=1+\varepsilon_{h}+\left(1+\varepsilon_{h}\right)\left(\varepsilon_{U}{ }^{2}+\varepsilon_{T}\right) \\
& \text { area velocity } \\
& \text { gradient gradient }
\end{aligned}
$$

The small parameters $\varepsilon_{U}$ and $\varepsilon_{T}$ are defined by

$$
\varepsilon_{U}^{2}=\frac{L_{A}}{L_{U}}, \quad \text { and } \quad \varepsilon_{T}=k L_{A}=2 \pi \frac{L_{A}}{L_{T}}
$$

Observed values for $\varepsilon_{U}, \varepsilon_{T}$ and $a \omega L_{A}(\bar{A} / \bar{b})^{-1} U^{-1}$ are presented in Table 4.1. The quantity $a \omega L_{A}(\bar{A} / \bar{b})^{-1} U^{-1}$ is nearly constant along channel because $a, \bar{A} / \bar{b}$ and $U$ are each nearly constant along channel.

Observations indicate that the above scalings are sensible (Table 4.1). In all three estuaries the quantity $a \omega L_{A}(A / b)^{-1} U^{-1}$ is $O(1)$, and the $O(\varepsilon)$ parameters are less to much less than one. Thus at first-order, only two of the terms in (4.2.4) are retained, namely the first term on the left hand side and the first term on the right hand side:

$$
\bar{b} \frac{\partial \zeta}{\partial t}=-\left(\frac{\mathrm{d} \bar{A}}{\mathrm{~d} x}\right) u .
$$

The scaling in this section has shown that in tidal estuaries of interest to this chapter, along-channel gradients in discharge are dominated by along-channel gradients in cross-sectional area. The dominance of gradients in cross-sectional area is due to strong channel convergence. The next most important contribution to the discharge gradient, at $O\left(\varepsilon_{T}\right)$, is from along-channel variations in the phase of tidal velocity. On a system-wide scale, the least important contribution to the discharge gradient, at $\mathrm{O}\left(\varepsilon_{U}{ }^{2}\right)$, is from alongchannel gradients in the amplitude of tidal velocity. This is because the tidal channels are near morphologic equilibrium. The above ordering is contrary to classic models of cooscillating tides in short prismatic channels, which suggest gradients in the amplitude of velocity should be most important and gradients in cross-sectional area should be least important. 


\subsubsection{Scaling of momentum}

For tides and estuaries described by (4.2.3) and (4.2.6), momentum may be expressed in terms of scales as:

$$
\omega U+\frac{U^{2}}{L_{A}}\left(\varepsilon_{a}^{2}+\varepsilon_{T}\right)=\frac{g a}{L_{A}}\left(\varepsilon_{a}^{2}+\varepsilon_{T}\right)+F .
$$

where $\varepsilon_{a}^{2}=L_{A} / L_{a}$ and $L_{a}$ is the e-folding length of tidal amplitude. Table 4.1 lists observed values for $\varepsilon_{a}$. At first order, (4.2.7) indicates that:

$$
U=\frac{\bar{b} a \omega L_{A}}{\bar{A}}=\frac{\bar{b}}{w} c \varepsilon_{h} \varepsilon_{T},
$$

where $c=\omega / k$. Dropping $\varepsilon_{a}^{2}$ relative to $\varepsilon_{T}$ in (4.2.10) and using (4.2.11) to eliminate $U$ then yields:

$$
\begin{aligned}
& \varepsilon_{T}+\frac{\bar{b}}{w} \varepsilon_{h} \varepsilon T^{2}=\frac{w}{\bar{b}} \frac{g \bar{h}}{c^{2}}+\frac{w}{\bar{b}} \frac{F}{\omega c \varepsilon_{h}} . \\
& \text { local advective pressure friction } \\
& \text { accel. accel. } \\
& \text { gradient }
\end{aligned}
$$

The speed of a linear shallow water gravity wave in a prismatic channel with intertidal storage and low friction is given by $c_{g}=(g \bar{h} w / \bar{h})^{1 / 2}$ (e.g., Robinson 1983). In the presence of friction, one might expect $c \leq c_{g}$, so the pressure gradient term in (4.2.12) should be $O(1)$ or greater. However the argument is not quite that straightforward. In a classic low friction, short prismatic channel, $c_{g}$ is not the phase speed of the single "observed" wave-form, which is termed $c$ in this study. Instead, $c_{g}$ is the phase speed of the individual progressive waves which propagate up- and down-estuary and which sum to form the single "observed" wave-form. Nonetheless, for the Delaware, Thames and Tamar, the third term in (4.2.12) is significantly greater than $\varepsilon_{T}$ (most significantly for the Tamar, least so for the Delaware). Table 4.1 displays the ratio of $\varepsilon_{T}$ to the pressure gradient term in (4.2.12).

The only term in (4.2.12) that can balance the pressure gradient at lowest order is the friction term. Thus an important result has been derived: In tidal channels with $\varepsilon_{T} \bar{h} c^{2} /(w g \bar{h}) \ll 1$, the lowest order balance must be between pressure gradient and friction. This conclusion has been reached without any à priori knowledge of the magnitude of the drag coefficient or of the tidal velocity. Furthermore, if $\varepsilon_{T} / \varepsilon_{h}=\mathrm{O}(1)$, the local acceleration 
term can be no more important than nonlinearities generated by finite amplitude effects in the continuity equation. Finally, (4.2.12) indicates that advective acceleration term is three orders in $\varepsilon$ smaller than friction. Thus when examining the system-wide tidal signature of strongly convergent tidal channels near morphologic equilibrium, advection may be neglected.

The dominant role for friction suggested above is in contrast with classic solutions for co-oscillating tides in prismatic channels which often neglect friction entirely. More realistic analytic approximations which consider convergent channel geometry in the presence of "strong" friction (Prandle and Rahman 1980; Jay 1991) have treated friction and local acceleration at the same order. The resulting solutions are expressed in terms of Bessel equations or repeated co-ordinate transformations which can interfere with conceptual interpretation. However, the scaling presented in this section suggests that by neglecting acceleration at first order, useful insights may be gained toward our understanding of tidal flow in strongly convergent tidal channels. This approach simplifies and clarifies the problem without sacrificing the fundamental physics.

\subsection{First-order solution}

\subsubsection{Form of the general solution}

If only first-order terms are retained in the continuity equation, then (4.2.1) may be re-expressed as:

$$
\bar{b} \frac{\partial \zeta}{\partial t}=-\left\{\frac{\mathrm{d} \bar{A}}{\mathrm{~d} x}\right\} u, \quad \text { or } \quad u=-\bar{b}\left\{\frac{\mathrm{d} \bar{A}}{\mathrm{~d} x}\right\}^{-1} \frac{\partial \zeta}{\partial t} .
$$

Equation (4.3.1b) guarantees that at first order $\zeta$ and $u$ will be $90^{\circ}$ out of phase, as in a standing wave. Dropping $\mathrm{O}(\varepsilon)$ terms, (4.2.2) may be written as:

$$
0=-g \frac{\partial \zeta}{\partial x}-F
$$

In one-dimensional numerical models of channelized tidal flow, the friction term is commonly formulated as (e.g., Speer and Aubrey 1985):

$$
F=\frac{c_{d}|u| u}{h_{R}},
$$


where $c_{d}$ is a non-dimensional drag coefficient, and $h_{R}$ is the hydraulic radius of the channel. In this study the friction term is linearized by expressing bottom friction as follows (e.g., Harleman 1966):

$$
F=r u, \text { with } \quad r \approx \frac{8}{3 \pi} \frac{c_{d} U}{\bar{h}},
$$

where $r$ is a constant friction factor, and $U$ is the "characteristic amplitude" of tidal velocity. It is reasonable to let $h_{R} \approx h$ for channels having $w \gg h$. The assumption that $r$ is constant in space is only approximately true due to along-channel variations in channel depth. The $8 /(3 \pi)$ in (4.3.4b) is derived from a truncated Fourier expansion of $\mid u l u$ in (4.3.3) (e.g., Speer and Aubrey 1985).

Substituting (4.3.1) into (4.3.2) then gives:

$$
\frac{\partial \zeta}{\partial x}=\frac{r \bar{b}}{g}\left\{\frac{\mathrm{d} \bar{A}}{\mathrm{~d} x}\right\}^{-1} \frac{\partial \zeta}{\partial t},
$$

which is a variable-coefficient, first-order wave equation for tidal elevation. The above governing equation is entirely different from the second-order wave equation which results from neglecting friction in a prismatic channel. It is also in contrast to the results of Friedrichs and Madsen (1992) who found tidal propagation in frictionally-dominated prismatic channels to be governed by a time-dependent diffusion equation.

By assuming a solution of the form $\zeta=\operatorname{Real}\left\{\xi(x) \mathrm{e}^{i \omega t}\right\},(4.3 .4)$ becomes:

$$
\frac{\mathrm{d} \xi}{\mathrm{d} x}=\frac{i \omega \bar{b} r}{g}\left\{\frac{\mathrm{d} \bar{A}}{\mathrm{~d} x}\right\}^{-1} \xi,
$$

or

$$
\ln \left(\frac{\xi}{\xi(0)}\right)=\frac{i r \omega}{g} \int_{x^{\prime}=0}^{x^{\prime}=x} \bar{b}\left(\frac{\mathrm{d} \bar{A}}{\mathrm{~d} x^{\prime}}\right)^{-1} \mathrm{~d} x^{\prime}
$$

The only boundary condition on (4.3.6) is $\xi(0)=a$, specified at the seaward end of the channel. Thus (4.3.6) may not be valid in the immediate vicinity of $x=L$, where it is presumed that zero tidal flow exists. However, the implied form of the velocity solution in (4.2.3), as well as the scaling inherent in (4.3.1), indicates that an upstream no-flow boundary cannot be important to the overall solution. Thus the potential loss of validity near $x=L$ is not a significant restriction. Since the dominant length scale of tidal discharge is $L_{A}$, a no-flow boundary condition at $x=L$ cannot be felt much seaward of $x / L$ 
$=1-L_{A} / L$, and in tidal estuaries of interest to this chapter, $L_{A} / L$ is significantly less than 1 (see Table 4.1).

Although there is zero tidal flow at $x=L$, there is usually finite fresh-water discharge in a real tidal estuary. The impact of fresh-water discharge at $x=L$ can be scaled as follows along a strongly convergent tidal channel: To maintain morphologic stability, the freshwater velocity at $x=L$ should be about the same as the tidal velocity in the seaward portion of the channel (see Chapter 3). Under "normal" conditions, then, the ratio of freshwater velocity to total velocity will have a decay length scale of $L_{A}$, and be negligible for $x / L<-1-L_{A} / L$. Wherever possible, observations used in this study are from "low" runoff conditions, further reducing the impact of freshwater discharge on tidal propagation. During high runoff or along channels that are not strongly convergent, river flow will have a more significant effect on tidal propagation throughout the channel (e.g., Godin 1991; Parker 1991). However these conditions are not a focus of this study.

\subsubsection{Exponential channel convergence}

Substituting the expressions for exponentially varying estuary width and channel cross-sectional area given by (4.2.6) into (4.3.7) gives:

$$
\ln \left(\frac{\xi}{a}\right)=-\frac{i r \omega}{g} \frac{b_{0} L_{A}}{A_{0}} \int_{x^{\prime}=0}^{x^{\prime}=x} \exp \left(x^{\prime} / L_{A}-x^{\prime} / L_{b}\right) d x^{\prime}
$$

which integrates to

$$
\ln \left(\frac{\xi}{a}\right)=-\frac{i r \omega}{g} \frac{b_{0} L_{A}}{A_{0}} \frac{L}{\lambda}\left(\mathrm{e}^{\lambda x / L}-1\right)
$$

where $\lambda=L / L_{A}-L / L_{b}$

Since it has already been shown that $L_{A} / L_{b} \approx 1$ (see Table 4.1), it is likely that $\lambda$ is small in estuaries of interest to this study. Observed $\lambda$ (Table 4.3) were calculated by fitting exponential relations to plots of $\bar{b} / \bar{A}$ versus $x / L$. Observed $\lambda$ is indeed less than one, and $\lambda x / L$ is even smaller since $x / L \leq 1$. If $\lambda x / L \ll 1$, then $\mathrm{e}^{\lambda x / L} \approx 1+\lambda x / L$, and (4.3.9) becomes:

$$
\ln \left(\frac{\xi}{a}\right)=-\frac{i r \omega}{g} \frac{b_{0} L_{A}}{A_{0}} x
$$

or 


$$
\xi=a \mathrm{e}^{-i k x},
$$

where

$$
k=\frac{2 \pi}{L_{T}}=\frac{r \omega}{g} \frac{b_{0} L_{A}}{A_{0}} \approx \frac{r \omega}{g} \frac{L_{A}}{(\bar{A} / \bar{b})},
$$

if $L_{A} / L_{b} \approx 1$.

Using (4.3.11), solutions for elevation and velocity become simply:

$$
\zeta=a \cos (\omega t-k x), \quad \text { and } \quad u=-U \sin (\omega t-k x) \text {, }
$$

where

$$
U=\frac{a \omega b_{0} L_{A}}{A_{0}} \approx \frac{a \omega L_{A}}{(\bar{A} / \bar{b})},
$$

is found by substituting (4.3.13a) into (4.3.1b). Equation (4.3.13) is entirely consistent with the form of solution which was assumed when scaling the problem in Section 4.2. Finally, the phase speed of (4.3.13), which is equal to the "observed" wave speed, is given by:

$$
c=\frac{\omega}{k}=\frac{g A_{0}}{r b_{0} L_{A}} \approx \frac{g(\bar{A} / \bar{b})}{r L_{A}} .
$$

Equation (4.3.15) indicates phase speed to be constant along the length of the channel.

\subsubsection{Discussion of first-order solution}

The first-order solution for strongly convergent channels near morphologic equilibrium diverges from the conventional view of co-oscillating estuary tides. From examining $(4.3 .13 \mathrm{a}, \mathrm{b})$ together, it is clear that $\zeta$ and $u$ are out of phase by $90^{\circ}$, as in a standing wave. Yet $(4.3 .13 \mathrm{a}, \mathrm{b})$ individually appear progressive. The first-order solution given by (4.3.13) is a forward propagating wave-form which is independent of the length of the tidal estuary -- in sharp contrast to the length-sensitive quarter-wave resonance of classic tidal estuary theory. Furthermore, the solution given by (4.3.13) is of constant amplitude, whereas the amplitude of a classical co-oscillating tide undulates along channel due to the interaction of incident and reflected waves.

The constant amplitude solution derived here is also significantly different from the frictionally-dominated solution found to hold for prismatic channels in Chapter 2. In Chapter 2, tidal phase was found to vary non-linearly along-channel and tidal amplitude was found to decay exponentially (see Figure 2.8 ). . In frictionally-dominated prismatic 
channels, frictional losses are such that a constant tidal amplitude cannot be maintained along the length of the channel. In strongly convergent channels, however, discharge decreases so quickly along-channel that a much smaller pressure gradient is required to maintain a given amplitude. Thus constant amplitude can be maintained at first order, even under conditions of frictional dominance.

The lack of a reflected wave in (4.3.13) indicates that the first-order solution does not "feel" landward conditions. Conceptually, the strong landward convergence of crosssectional area quickly dissipates any seaward propagating information. Even if a reflected wave were somehow included in (4.3.13), perhaps to improve the solution in the immediate vicinity of an upstream boundary, the reflected wave would only propagate over a distance of order $L_{A}$ before becoming negligible in comparison to the landward propagating wave. This result has important ramifications concerning the potential effect of tidal barriers. In strongly convergent channels, the installation of a tidal barrier at $x=x_{b}$ should have a minimal effect on the tidal signal seaward of $x=x_{b}-L_{A}$. This finding is consistent with Prandle and Raman (1980) who examined the effect of tidal barriers using Bessel function solutions. Upon introduction of barriers into strongly convergent channels, they found the amplitude and phase of elevation and velocity to be altered by only a few percent outside the immediate vicinity of the barrier.

The velocities predicted by (4.3.14) for the Delaware, Thames and Tamar (Table 4.3) are somewhat higher than the observed values. This is partly because predicted $U$ is averaged over $w$, whereas observed $U$, as presented in other investigations (Harleman 1966; Chantler 1974; Uncles et al. 1985), is averaged over all of $\bar{b}$ (see Figure 4.1). Predicted and observed velocity agree best for the Delaware, for which $w / \bar{b} \approx 1$, and worst for the Tamar, for which $w / \bar{h}$ is smallest. According to Uncles et al. (1985), maximum current speeds in the deep central channel of the Tamar are on the order of $1 \mathrm{~m} / \mathrm{s}$, which is more consistent with the predicted value. Comparison to observed $U$ in all three estuaries is further complicated by differences in the amplitude of the forcing tide during the periods of velocity observation and during the periods of elevation observation -- which are generally neither synchronous nor of equal duration. Also, velocity in general is much more sensitive than tidal elevation to measurement location and to variations in channel shape away from an idealized geometry. Considering these limitations, differences between the magnitude of observed and predicted $U$ do not undermine the applicability of the first-order theory. 
Equations (4.3.12), (4.3.14) and (4.3.4b) allow the wavelength of the dominant tidal frequency to be predicted with $c_{d}$ as the only independent parameter:

$$
L_{T}=\frac{3 \pi^{2}}{4} \frac{g(\bar{A} / \bar{b})^{2}}{c_{d} \varepsilon_{h} \omega^{2} L_{A}{ }^{2}} .
$$

Alternatively, the observed value of $L_{T}$ can be used to solve for the unknown drag coefficient. The resulting "observed" values for $c_{d}$ are displayed in Table 4.3. These $c_{d}$ values are consistent with those previously employed in 1-D numerical models of strongly convergent tidal estuaries. For example, in modeling the Delaware, Parker (1984) allowed $c_{d}$ to vary between $2.0 \times 10^{-3}$ and $3.7 \times 10^{-3}$, and in modeling the Tamar, Uncles and Stephens (1989) used $c_{d}=1.6 \times 10^{-3}$. (Prandle (1980) did not discuss his choice of $c_{d}$ for his model of the Thames). However these drag coefficients are several times smaller than $c_{d}$ values which have been used in 1-D representations of other embayments such as Great Bay, New Hampshire (Swift and Brown 1983) and Nauset Harbor, Massachusetts (Aubrey and Speer 1985; Speer and Aubrey 1985).

Embayments represented by $c_{d}$ values which are $\mathrm{O}\left(10^{-2}\right)$, such as Great Bay and Nauset contain sharp channel bends and sudden along-channel expansions in crosssectional area (Aubrey and Speer 1984; Swift and Brown 1983). At these sudden transitions, transfer of momentum occurs from organized along-channel flow to large turbulent eddies. On a system-wide scale, these eddies may be justifiably modeled as frictional losses and lead to large values of $c_{d}$. At both Nauset and Great Bay, sudden morphologic irregularities are largely a product of antecedent geology -- unevenly filled depressions in glacial outwash and ice contact deposits and at Nauset (Oldale 1979) and a rock-walled valley formed along a major fold axis at Great Bay (Haug 1969). Thus neither embayment can be described as being near morphologic equilibrium.

Equations (4.3.15) and (4.3.16) predict wave speeds and lengths which are the same order as those predicted by the frictionless shallow-water gravity wave speed for systems without tidal flats, namely, $c_{g}=(g \bar{h})^{1 / 2}$. Table 4.3 lists predictions for the frictionless wave length, $L_{g}$ (neglecting the role of intertidal storage), for the $\mathrm{M}_{2}$ tide in the Delaware, Thames and Tamar. The similar magnitude of $L_{T}$ and $L_{g}$ has no doubt exacerbated the historic mis-interpretation of tidal propagation in highly convergent channels as being well-described by classical damped co-oscillation. 


\subsection{Second-order solution}

\subsubsection{Derivation of governing equation}

At second-order (see Table 4.2), (4.2.7) indicates that the following terms are kept in the continuity equation:

$$
\left(1+\varepsilon_{b} \frac{\zeta}{a}\right) \bar{b} \frac{\partial \zeta}{\partial t}=-\left(1+\varepsilon_{h} \frac{\zeta}{a}\right)\left(\frac{\mathrm{d} \bar{A}}{\mathrm{~d} x}\right\} u-\bar{A} \frac{\partial u}{\partial x} .
$$

The first-order solutions for $\zeta$ and $u$, i.e. (4.3.13), may be substituted into the secondorder terms of (4.4.1), giving

$$
b_{0}\left\{1+\varepsilon_{b} \cos (\omega t-k x)\right\} \frac{\partial \zeta}{\partial t}=\left\{1+\varepsilon_{h} \cos (\omega t-k x)\right\} \frac{A_{0}}{L_{A}} u+A_{0} i k u,
$$

where the exponential expressions for $\bar{b}$ and $\bar{A}(4.2 .6 \mathrm{a}, \mathrm{b})$ with $L_{A} \approx L_{b}$ have also been used. Solving for $u$ in (4.4.2) then gives, to $\mathrm{O}(\varepsilon)$,

$$
u=\{1-\gamma \cos (\omega t-k x)\}\left(1-i \varepsilon_{T}\right) \frac{L_{A} b_{0}}{A_{0}} \frac{\partial \zeta}{\partial t},
$$

where $\gamma=\varepsilon_{h}-\varepsilon_{b}$. Observed values of $\gamma$ are listed in Table 4.3.

Retaining only those terms of (4.2.11) which are significant at second order, momentum may be expressed as

$$
\frac{\partial u}{\partial t}=-g \frac{\partial \zeta}{\partial x}-r u
$$

Second-order effects arising from the friction term will not be considered in this study, even though the formulation of (4.3.3) does generate tidal harmonics. If the friction term is formulated in the common manner of (4.3.3), even harmonics will be produced by tidal modulation of $h_{R}$, and odd harmonics will be produced by $u l u l$ (e.g., Parker 1991; Friedrichs and Madsen 1992). The production of second-order tidal harmonics by (4.3.3) has been demonstrated conclusively by numerical solutions which include it (e.g., Speer and Aubrey 1985). However, field observations of tides in channels have not demonstrated that the formulation in (4.3.3) accurately reproduces observed nonlinearities in natural tidal channels. 
For example, one-dimensional numerical solutions for tides in channels which use (4.3.3) generally do a poor job of reproducing along-channel variations in $M_{6}$ (e.g., Prandle 1980; Parker 1984; Friedrichs and Madsen 1992). There is little doubt that the friction term is the major source of $\mathrm{M}_{6}$ production within tidal channels. However, (4.3.3) questionably assumes that the drag coefficient is time-invariant. Field observations suggest that in energetic tidal flows $c_{d}$ can be a complex function of tidal height, tidal velocity, and flow direction (Wallis and Knight 1984; Lewis and Lewis 1987; Weisman et al. 1990). By applying observations to the terms in the 1-D momentum equation and solving for the drag coefficient, Lewis and Lewis (1984) and Weisman et al. (1990) found $c_{d}$ to vary by threeto-four times over the tidal cycle, while Wallis and Knight (1987) observed an order of magnitude variation in $c_{d \cdot}$. Thus the true $\mathrm{M}_{6}$ produced by friction may not closely resemble that predicted by (4.3.3) unless $c_{d}$ is more properly represented as varying in time.

Tidal modulation of $c_{d}$ will affect even harmonics directly. For example, if modulation of $c_{d}$ is out of phase with modulation of $h_{R}$, net production of even harmonics may be reduced in real tidal channels. This may be partly why a one-dimensional numerical model of the Thames which used (4.3.3) (Prandle 1980) predicted $\mathrm{M}_{4}$ amplitudes several times larger than the observed values. Yet in a similar one-dimensional model of the Delaware, Parker (1984) was able to reproduce along-channel variations in $\mathrm{M}_{4}$ amplitude quite well (although he presented no model output of $\mathrm{M}_{4}$ phase). When Parker (1984) examined the various sources of $M_{4}$ in the model, he found that the $M_{4}$ from non-linear continuity was 3.7 times larger than the $\mathrm{M}_{4}$ from friction and more than ten times larger than the $\mathrm{M}_{4}$ from advection. He stated that the various contributions to $\mathrm{M}_{4}$ had different phases, and that the total $\mathrm{M}_{4}$ amplitude was less than the sum of the individual contributions. Thus his model might have reproduced $\mathrm{M}_{4}$ just as well without including tidal modulation of $h_{R}$ in the friction term.

The only remaining second-order term in (4.4.4) is the local acceleration term. Substitution of the first-order solution into this term gives

$$
i \omega u=-g \frac{\partial \zeta}{\partial x}-r u
$$

or

$$
\frac{\partial \zeta}{\partial x}=-\frac{r}{g}\left(1+i \varepsilon_{\omega}\right) u
$$

where $\varepsilon_{\omega}=\omega / r$. Using (4.3.4b) and (4.3.14), $\varepsilon_{\omega}$ can be re-expressed as 


$$
\varepsilon_{\omega}=\frac{3 \pi(\bar{A} / \bar{b})}{8 c_{d} \varepsilon_{h} L_{A}}
$$

This parameter, which determines the importance of acceleration relative to bottom friction, is independent of both tidal velocity and tidal frequency. Table 4.3 indicates $\varepsilon_{\omega}$ is indeed less to much-less than one in all three estuaries of interest. The values given for $\varepsilon_{\omega}$ in Table 4.3 are similar to the values for the ratio $\varepsilon_{T} \bar{b} c^{2} /(w g \bar{h})$ given in Table 4.1 , which also predicted the relative unimportance of acceleration.

Substituting (4.4.3) into (4.4.6) to eliminate $u$ yields a single equation for $\zeta$ :

$$
\frac{\partial \zeta}{\partial x}=-\{1-\gamma \cos (\omega t-k x)\} \frac{(1+i \mu)}{c} \frac{\partial \zeta}{\partial t}
$$

where $\mu=\varepsilon_{\omega}-\varepsilon r$, and $c$ is the first-order wave speed given by (4.3.15). Observed values for $\mu$ are given in Table 4.3. Eq. (4.4.8), like (4.3.5), is a first-order wave equation with only one boundary condition, namely $\zeta=a \cos \omega t$ at $x=0$.

\subsubsection{General solution}

The second-order solution is derived in a manner similar to Friedrichs and Madsen (1992). The solution is formally perturbed in time only, and the second-order spatial dependence is treated kinematically. The cosine term in (4.4.8) is evaluated at $x=x_{0}$, where $0 \leq x_{0} \leq L$ is some representative location along the channel. This greatly simplifies the form of the second-order solution without significantly affecting its accuracy for relatively short systems. More formal perturbations in time and space (e.g., Shetye and Gouveia 1992) produce a myriad of additional terms which obscure the most important non-linear mechanisms and hinder physical interpretation.

Next the time variable is transformed from $t$ to $\tau$, with

$$
\omega \tau=\omega t+\gamma \sin \left(\omega t-k x_{0}\right)
$$

Then by using the relation $\partial \zeta / \mathrm{d} t=\partial \zeta / \partial \tau \mathrm{d} \tau / \mathrm{d} t,(4.4 .8)$ beccmes

$$
\frac{\partial}{\partial x} \zeta(x, \tau)=-\frac{(1+i \mu)}{c} \frac{\partial}{\partial \tau} \zeta(x, \tau),
$$

which has time-independent coefficients. 
The formulation of $\tau$ in Friedrichs and Madsen (1992) differs slightly by considering non-linearities produced by the friction term. In their paper, $\gamma=5 / 3 \varepsilon_{h}-\varepsilon_{b}$ instead of $\varepsilon_{h}-\varepsilon_{b}$. Friedrichs and Madsen included frictional non-linearities in their analytic discussion in order to make diagnostic comparisons to numerical solutions which also included non-linear friction. Here it is argued that field observations do not support inclusion of non-linear friction in the form of (4.3.3). The difference in approach between here and Friedrichs and Madsen affects the magnitude of the non-linear mechanism. However it does not fundamentally affect the interpretation of the processes most responsible for generating harmonics.

Since (4.4.10) is linear, its solution may be expressed as a sum of individual terms, each satisfying (4.4.10) at the single frequency $m \omega$. Thus the general solution to (4.4.10) at each frequency takes the form:

$$
\zeta_{m}(x, \tau)=\alpha_{m} \xi_{m}(x) \exp (i m \omega \tau)
$$

where $\left|\xi_{m}\right|=1$ at $x=0$.

In order to apply the boundary condition at $x=0$ to $(4.4 .11)$, the boundary condition must be transformed from $t$ to $\tau$. Utilizing the definition of $\tau$, trigonometric identities, and approximations valid at $\mathrm{O}(\varepsilon$ ) (for details see Appendix 2), it can be shown that

$$
\zeta(x=0, t)=a \cos \omega t \rightarrow \zeta(x=0, \tau)=a \sum_{m=0}^{2} Z_{m} \cos \left(m \omega \tau-\theta_{m}\right)
$$

where $Z_{0}=-Z_{2}=\gamma / 2, Z_{1}=1,-\theta_{0}=\theta_{2}=k x_{0}$, and $\theta_{1}=0$. Applying (4.4.12) to (4.4.11) gives $\alpha_{m}=a Z_{m} \mathrm{e}^{-i \theta m}$, and the solution in the $\tau$ domain becomes

$$
\zeta_{m}(x, \tau)=a Z_{m} \xi_{m}(x) \exp \left(i m \omega \tau-\theta_{m}\right) .
$$

Next, (4.4.13) is transformed back to the $t$ domain. For $m=0$ and $m=2,(4.4 .13)$ is already $\mathrm{O}(\varepsilon)$, so by discarding $\mathrm{O}(\varepsilon)^{2}$ terms, (4.4.13) becomes $\zeta_{m}(t, x)=a Z_{m} \xi_{m} \exp$ (im $\omega t-\theta_{m}$ ) directly. In order to transform the $m=1$ case, however, $\exp (i \omega \tau)$ must be reexpressed in terms of $t$. Utilizing the definition of $\tau$, trigonometric identities, and approximations valid at $\mathrm{O}(\varepsilon)$, it can be shown (see Appendix 2) that

$$
\exp i \omega \tau=-\frac{\gamma}{2} \exp i k x_{0}+\exp i \omega t+\frac{\gamma}{2} \exp i\left(2 \omega t-k x_{0}\right) .
$$


The full solution in the $t$ domain may may then be expressed as a sum of single frequency components, i.e.:

$$
\zeta(x, t)=a \sum_{m=0}^{2} \eta_{m}(x, t)
$$

where

$$
\begin{gathered}
\eta_{0}=\frac{\gamma}{2}\left(\xi_{0}-\xi_{1}\right) \mathrm{e}^{i k x_{0}}, \\
\eta_{1}=\xi_{1} \mathrm{e}^{-i \omega t}, \\
\eta_{2}=\frac{\gamma}{2}\left(\xi_{1}-\xi_{2}\right) \mathrm{e}^{i\left(2 \omega t-k x_{0}\right)} .
\end{gathered}
$$

By substituting (4.4.13) into (4.4.10), the governing equations for $\xi_{m}(x)$ are seen to be

$$
\frac{\mathrm{d}}{\mathrm{d} x} \xi_{m}(x)=-\frac{(1+i \mu)}{c} i m \omega \xi_{m}(x)
$$

Eq. (4.4.17) integrates easily to

$$
\xi_{m}=\mathrm{e}^{\mu m k x} \mathrm{e}^{-i m k x},
$$

where $k=\omega / c$. Substituting (4.4.18) into (4.4.15) - (4.4.16) then gives the following, compact second-order solution for tidal elevation:

$$
\begin{aligned}
& \frac{\zeta}{a}=\frac{\gamma}{2}\left\{1-\mathrm{e}^{\mu k x} \mathrm{e}^{-i k x}\right\} \mathrm{e}^{i k x_{0}}+\mathrm{e}^{\mu k x} \mathrm{e}^{i(\omega t-k x)} \\
& +\frac{\gamma}{2}\left\{\mathrm{e}^{\mu k x} \mathrm{e}^{-i k x}-\mathrm{e}^{2 \mu k x} \mathrm{e}^{-2 i k x}\right\} \mathrm{e}^{i\left(2 \omega t-k x_{0}\right)}
\end{aligned}
$$

The second-order solution for velocity is found by substituting (4.4.15) - (4.4.16) into (4.4.3), giving,

$$
u=i U\left\{1-\gamma \cos \left(\omega t-k x_{0}\right)\right\}\left(1-i \varepsilon_{T}\right)\left\{\xi_{1} \mathrm{e}^{i \omega t}+\gamma\left(\xi_{1}-\xi_{2}\right) \mathrm{e}^{i\left(2 \omega t-k x_{0}\right)}\right\},
$$

where $U$ is the first-order velocity amplitude given by (4.3.15), and $x_{0}$ has been used for consistency with the solution for elevation. Multiplying out (4.4.20), dropping terms that are $O(\varepsilon)^{2}$, and using (4.4.18) to eliminate $\xi_{m}$ then gives the following real solution for tidal velocity: 


$$
u=U\left(V_{0}+V_{1}+V_{2}\right)
$$

where

$$
\begin{gathered}
V_{0}=-\frac{\gamma}{2} \mathrm{e}^{\mu l \alpha} \sin k\left(x-x_{0}\right), \\
V_{1}=-\mathrm{e}^{\mu k x}\left\{\sin (\omega t-k x)-\varepsilon_{T} \cos (\omega t-k x)\right\}, \\
V_{2}=-\frac{\gamma}{2}\left\{\mathrm{e}^{\mu \alpha x} \sin \left(2 \omega t-k x-k x_{0}\right)-2 \mathrm{e}^{2 \mu k x} \sin \left(2 \omega t-2 k x-k x_{0}\right)\right\} .
\end{gathered}
$$

\subsection{Discussion of second-order solution for elevation}

\subsubsection{Dominant tidal frequency}

The second-order solution at the dominant tidal frequency is given by the real part of the second term in (4.4.19), namely

$$
\eta_{1}=\mathrm{e}^{\mu k x} \cos (\omega t-k x) .
$$

Equation (4.5.1) describes a purely progressive wave with the same phase speed as the first-order solution. However the amplitude which is modulated by $\mathrm{e}^{\mu \mathrm{kx}}$, where $\mu=\varepsilon_{\omega}$ $\varepsilon_{T}$. This result is consistent with the observations in Figure 4.2 (especially for the Delaware) which indicate along-channel phase variation to be more strongly linear than along-channel amplitude variation.

The exponential modulation of tidal amplitude at second-order is due to the combined, partially-cancelling effects of (i) non-zero acceleration, which is represented by $\varepsilon_{\omega}$, and (ii) limited convergence, which is represented by $\varepsilon_{T}$. Conceptually, non-zero acceleration increases tidal amplitude by allowing a dynamic along-channel convergence of energy. This effect is analogous to Green's law in the frictionless, weak convergence limit and has previously been termed "topographic funneling" (Jay 1992). Limited convergence (i.e., a tendency toward a prismatic channel) decreases tidal amplitude because a larger pressure gradient is required maintain a given amplitude along a prismatic channel. Acceleration effects do not produce a reflected wave because the form of the governing equation (4.4.8) allows information to travel only landward.

If $\mu>0$, then acceleration effects overcome damping due to limited convergence, and tidal amplitude grows along channel. If $\mu<0$, damping due to limited convergence 
overshadows acceleration, and amplitude decays. Because $\mu$ is smaller than either $\varepsilon_{\omega}$ or $\varepsilon_{T}$, the second-order solution for elevation is more like the first-order solution than might be predicted from the size of $\dot{\varepsilon}_{\omega}$ or $\varepsilon_{T}$ alone. Thus the applicability of the first-order scaling, if based on the size of $\mu$, is extended. If $\mu=0$, these two second-order effects cancel entirely, and the solution for $\eta_{1}$ is identical to the first-order case. Jay (1991) found a similar result via a more complex, modified Green's function solution. Under conditions he termed "critical convergence", Jay also found local acceleration to cancel with a convergence-related term from the continuity equation, leaving an effective balance between friction and pressure gradient.

The observed exponential variation in the amplitude of tidal elevation, described by the e-folding length $L_{a}$, should be related to $\mu$ as follows:

$$
\mu=\frac{1}{2 \pi} \frac{L_{T}}{L_{a}} .
$$

Table 4.3 lists $L_{T} /\left(2 \pi L_{a}\right)$ for the Delaware, Thames and Tamar. The correspondence to $\mu=$ $\varepsilon_{\omega}-\varepsilon_{T}$ is consistent within error bars for the Delaware and Thames. Application of (4.5.2) to the Tamar is inconsistent with $\mu=\varepsilon_{\omega}-\varepsilon_{T} . \mu=\varepsilon_{\omega}-\varepsilon_{T}=0.15 \pm 0.06$ indicates tidal amplitude should increase along the Tamar, while observed along-channel tidal amplitude clearly decreases in Figure 4.2. However, the observed spring tidal amplitude along the Tamar may not be entirely dynamic. Since $\varepsilon_{h}=a / \bar{h} \approx 1$ along the Tamar, the channel is nearly dry at low tide, and low water elevation is largely constrained by the elevation of the bottom. This phenomenon has been discussed in detail by Speer et al. (1990).

Observed spring tidal elevations along the Tamar were recorded relative to a single horizontal datum (George 1975). Thus it is possible to examine along-channel variations in the elevation of high water. If the "dynamic" tidal amplitude along the Tamar is defined as local high water elevation minus mid-tide elevation at $x=0$, then tidal amplitude is found to increase along channel (Figure 4.4) with an e-folding length-scale of $L_{a}=190 \pm 20 \mathrm{~km}$. Using this new estimate of $L_{a},(4.5 .2)$ gives $\mu=0.23 \pm 0.02$, which is consistent with $\mu=$ $\varepsilon_{\omega}-\varepsilon_{T}$ to within error bars. Figure 4.4 displays the observed "dynamic" amplitudes along the Tamar with the second-order solution given by (4.5.1).

The prediction of second-order amplitude and phase variation along the Delaware and Thames can be improved by dividing up each observed channel into several individual, exponentially-varying segments. Because (4.5.1) describes a unidirectional wave-form, the change in amplitude and phase along segment $j$ is given directly by 


$$
\left|\eta_{1, j}\right|=\left|\eta_{1, j-1}\left(L_{j-1}\right)\right| \mathrm{e}^{\mu_{j} k_{j} x_{j}}, \quad \varphi_{\eta 1, j}=k_{j} x_{j}+\varphi_{\eta 1, j-1}\left(L_{j-1}\right)
$$

where $\mu_{j}=\varepsilon_{\omega j}-\varepsilon_{T j}$ and $k_{j}$ are calculated from the along-channel geometry of each segment, and $x_{j}=0$ at the beginning of each segment. The boundary condition for each segment is simply the amplitude and phase at the end of the previous segment. Equation (4.4.7) is used to determine $\varepsilon_{\omega j}$. The only freely determined parameter is $c_{d}$, which is used to determine $\varepsilon_{T j}$ and $k_{j}$ via (4.3.16).

The along-channel variations in observed $\left|\eta_{1}\right|$ and $\varphi_{\eta 1}$ (see Figure 4.2) suggest that an improved representation of the Thames requires at least two exponentially-fit segments (to capture growth of tidal amplitude followed by decay), whereas the Delaware requires at least three (to capture growth, followed by weak decay, followed by subsequent growth). There are insufficient geometric observations along the Tamar to merit its division into segments. Figure 4.4 displays observations and second-order solutions for $a\left|\eta_{1}\right|$ and $\varphi_{\eta 1}$ along the Thames and the Delaware. Table 4.4 displays values of $\mu_{j}, L_{T j}$ and $L_{A j}$ calculated for each segment. Where $\mu_{j}>0$, tidal amplitude locally increases with distance along channel, and where $\mu_{j}<0$, tidal amplitude locally decreases.

The observed undulations in along-channel tidal amplitude and observed changes in the slope of tidal phase displayed in Figure 4.4 are primarily due to changes in the rate of convergence of channel geometry with distance. They are not due to interactions between a single landward-propagating incident wave and a single seaward-propagating reflected wave as commonly presumed through application of classical damped co-oscillation. Equation (4.5.3) reproduces these undulations yet includes no distinct reflected wave. Acceleration effects which do occur are more correctly interpreted as a dynamic convergence of energy propagating in a landward direction as part of the single, overall wave-form.

In computing the segmented solution, the drag coefficient was held constant at $c_{d}=$ $3.0 \times 10^{-3}$ along the Thames and $c_{d}=1.7 \times 10^{-3}$ along the Delaware. These "best-fit" $c_{d}$ values for the segments of each estuary are smaller than the corresponding $c_{d}$ values for each entire estuary. This is not surprising, for acceleration should become increasingly important at smaller scales. At very small scales (e.g., channel meanders, expansions, or pools) advective acceleration will also play a role. Thus the smaller $c_{d}$ values for individual segments do not undermine our earlier assertion that, on a system-wide scale, friction dominates acceleration at first-order. Rather, the excellent agreement between observations 
and theory displayed in Figure 4.4 indicates that the second-order theory presented above provides a simple yet powerful explanation of tidal propagation in strongly convergent channels near morphologic equilibrium.

\subsubsection{Second harmonic}

From (4.4.19), the amplitude ratio of the second-to-first harmonic is given by

$$
\left|\frac{\eta_{2}}{\eta_{1}}\right|=\frac{|\gamma|}{2}\left|1-\mathrm{e}^{\mu k x} \mathrm{e}^{-i k x}\right|=\frac{|\gamma|}{2}\left(1-2 \mathrm{e}^{\mu k x} \cos k x+\mathrm{e}^{2 \mu k x}\right)^{1 / 2} .
$$

Equation (4.5.4) is independent of the choice of $x_{0}$ used in evaluating (4.4.19). For the case of $\mu=0,(4.5 .4)$ reduces to

$$
\left|\frac{\eta_{2}}{\eta_{1}}\right|=\frac{|\gamma|}{\sqrt{2}}(1-\cos k x)^{1 / 2} .
$$

Figure 4.5 compares (4.5.4) and (4.5.5) to observations of $\left|\eta_{2} / \eta_{1}\right|$ along the Delaware (Parker 1984), Thames (Prandle 1980) and Tamar (George 1975). In calculating (4.5.4) (4.5.5) for the Tamar, the "dynamic" amplitude discussed in Section 4.5.1 was applied, which gives $\gamma=0.75 \pm 0.06$. For the Thames and Tamar there is significant disagreement between theory and observations near $x / L=0$ where forcing was assumed to occur only at the dominant tidal frequency. In reality there is a finite $\eta_{2}$ forcing at the mouth of each system. In the inner estuary, where internally generated $\eta_{2}$ is presumed to dominate, the agreement between theory and observations is better.

The undulations in $\left|\eta_{2} / \eta_{1}\right|$ along the Delaware may be partially explained by changes in the rate of convergence of channel geometry with distance. Equation (4.5.4) indicates that $\left|\eta_{2} / \eta_{1}\right|$ grows along-channel more quickly when $\mu>0$ and and more slowly when $\mu<$ 0. Observations displayed in Figure 4.5a suggests that growth in $\left|\eta_{2} / \eta_{1}\right|$ is more pronounced in the first and third "segments" of the Delaware where $\mu$ is positive, and less so in the second segment where $\mu$ is negative (see Table 4.4). Since $\left|\eta_{2} / \eta_{1}\right|$ is scaled by $\gamma=$ $\varepsilon_{h}-\varepsilon_{b}$, the undulations may be further enhanced by along-channel variations in $\gamma$, which was set equal $\varepsilon_{h}$ for the Delaware. $\varepsilon_{h}=a / \bar{h}$ increases rapidly in the innermost Delaware estuary, reaching $\sim 0.2$ at $x / L=0.9$ and $\sim 0.5$ at $x / L=1$ (Parker 1984). This increase in local $\gamma$ coincides with the sharp increase in observed $\left|\eta_{2} / \eta_{1}\right|$ (Figure 4.5a).

Equations (4.5.4) and (4.5.5) reproduce observed $\mid \eta_{2} / \eta_{1} l$ in the inner Thames more closely than the 1-D numerical modeling of Prandle (1980), who predicted $\left|\eta_{2} / \eta_{1}\right|=0.12$ at 
$x=0.6$ (versus an observed value of 0.03 ). To calculate $\left|\eta_{2} / \eta_{1}\right|$ for the Thames, the geometry of the first "segment" in Table 4.4 was used since all the available observations of $\eta_{2}$ are for $x / L<0.8$. The poor performance of the numerical solution is likely the result of two factors. First, Prandle included tidal modulation of channel depth in the friction term. As argued in Section 4.4.1, the inclusion of time-varying depth in the friction term is probably not justified unless the time-dependence of $c_{d}$ can be well constrained.

Second, and perhaps more importantly, Prandle (1980) did not distinguish between subtidal channels and intertidal flats in his numerical model. According to the analytic theory developed here, $\left|\eta_{2} / \eta_{1}\right|$ is scaled by $\gamma=\varepsilon_{h}-\varepsilon_{b}$, where $\varepsilon_{b}$ measures the relative size of tidal variations in estuary width due to intertidal flats. If $\varepsilon_{b}$ had been neglected, and it were assumed that $\gamma \approx \varepsilon_{h}$, then the present prediction of $\left|\eta_{2} / \eta_{1}\right|$ for the Thames would have increased by three-fold. This may also explain why (4.5.4) - (4.5.5) over-predict $\left|\eta_{2} / \eta_{1}\right|$ in the inner Delaware where a small, but certainly non-zero, region of intertidal storage exists.

The phase of the second harmonic can also be determined from (4.4.19). If $\eta_{2}$ is expressed as $\left|\eta_{2}\right| \exp i\left(2 \omega t-\varphi_{\eta 2}\right)$, then after some algebra (see Appendix 3) $\varphi_{\eta 2}$ is given by

$$
\varphi_{\eta 2}=\arctan \left\{\frac{\sin \left(k x+k x_{0}\right)-\mathrm{e}^{\mu k x} \sin \left(2 k x+k x_{0}\right)}{\cos \left(k x+k x_{0}\right)-\mathrm{e}^{\mu k x} \cos \left(2 k x+k x_{0}\right)}\right\}+\pi \delta\left(1, \frac{-\gamma}{|\gamma|}\right),
$$

where $\delta(m, l)=1$ if $m=l, \delta=0$ otherwise. For $\mu=0$, trigonometric identities may be used (see Appendix 3) to show that (4.5.6) reduces to

$$
\varphi_{\eta 2}=\frac{3}{2} k x+k x_{0}-\frac{\pi}{2} \frac{\gamma}{|\gamma|} .
$$

The phase of $\eta_{2}=\left|\eta_{2}\right| \exp i\left(2 \omega t-\varphi_{\eta 2}\right)$ relative to $\eta_{1}=\left|\eta_{1}\right| \exp i\left(\omega t-\varphi_{\eta 1}\right)$ is defined as $2 \varphi_{\eta 1}-\varphi_{\eta 2}$ (Aubrey and Speer 1985). So from (4.5.7) and (4.5.1), for $\mu=0$,

$$
2 \varphi_{\eta 1}-\varphi_{\eta 2}=\frac{\pi}{2} \frac{\gamma}{|\gamma|}+\frac{k x}{2}-k x_{0} .
$$

The solution for $2 \varphi_{\eta 1}-\varphi_{\eta 2}$, unlike that for $\left|\eta_{2} / \eta_{1}\right|$, is a function of $x_{0}$. Figure 4.6 compares (4.5.8) to observations of $2 \varphi_{\eta 1}-\varphi_{\eta 2}$ using both $x_{0}=0$ and $x_{0}=L / 2$. Observations for the Delaware and Tamar are again from Parker (1984) and from George (1975). Phase information for $\varphi_{\eta 1}$ and $\varphi_{\eta 2}$ presented by Prandle, however, are relative to separate constants and thus in a form inconsistent with the application of (4.5.8). Less extensive observations from Hunt (1964) are used instead. 
As was the case for $\left|\eta_{2} / \eta_{1}\right|$, observed $2 \varphi_{\eta 1}-\varphi_{\eta 2}$ near the mouth of each estuary is apparently influenced by existing $\eta_{2}$ forcing at the mouth, and agreement between observations and theory is better within the estuary. Equation (4.5.8) reproduces observations about as well with $x_{0}=0$ or $x_{0}=L / 2$. Predictions are less sensitive to $x_{0}$ and observed $2 \varphi_{\eta 1}-\varphi_{\eta 2}$ is reproduced more closely in the inner. Thames and Tamar than in the Delaware. This is not surprising, since the Tamar $(k L=0.48)$ and the Thames $(k L=0.96$ if based on segment 1$)$ are much shorter than the Delaware $(k L=3.7)$, and the use of a representative $x_{0}$ is asymptotically valid only for small $k L$. Nonetheless, (4.5.8) captures the general sense of tidal asymmetry even in a system as long as the Delaware.

The phase of $\eta_{2}$ relative to $\eta_{1}$ indicates whether an asymmetric tidal cycle has a rising tide of shorter duration $\left(0^{\circ}<2 \varphi_{\eta 1}-\varphi_{\eta 2}<180^{\circ}\right)$ or a falling tide of shorter duration $\left(-180^{\circ}<2 \varphi_{\eta 1}-\varphi_{\eta 2}<0^{\circ}\right.$ ) (Aubrey and Speer 1985). For $k L \leq \pi$, (4.5.8) predicts the tide will be shorter-rising if $\gamma$ is positive and shorter-falling if $\gamma$ is negative. The sign of $\gamma$ can be expected to determine the sense of distortion in the same way for longer tidal channels, but the above theory is based on a short channel asymptote. Friedrichs and Madsen (1990) found asymmetry in frictionally-dominated prismatic channels to depend on $\gamma$ in the same fashion (although their $\gamma$ was defined slightly differently).

The mechanism by which $\gamma$ controls asymmetry in strongly convergent channels is explained conceptually if the tidal wave speed given by (4.3.15) is approximated as follows:

$$
c(t)=\frac{g A(t)}{r L_{A} b(t)}=\frac{g w}{r L_{A}} \frac{\bar{h}\left(1+\varepsilon_{h} \zeta\right)}{\bar{b}\left(1+\varepsilon_{b} \zeta\right)}=\frac{g w \bar{h}}{r L_{A} \bar{b}}(1+\gamma \zeta) .
$$

If $\gamma>0$, the wave speed given by (4.5.9) is greater around high water, when $\zeta>0$, than it is around low water, when $\zeta<0$. High water moves faster along the tidal channel, "catching-up" with low water, and causing the rising tide to be of shorter duration. If $\gamma<$ $0, c(t)$ is greater around low water, low water moves faster along the channel, and the result is a shorter falling tide.

In the past, observations of shorter-rising tides in strongly convergent channels have been attributed to time-variation of the frictionless shallow water gravity wave speed, $c_{g}=(g h)^{1 / 2}$ (Wright et al. 1975; McDowell and O'Connor 1977; Allen et al. 1980). Given the historical emphasis on low-friction co-oscillation in tidal channels, it is not surprising that previous authors have looked to the frictionless wave speed for an explanation. A 
time-varying $c_{g}$ can give results which are qualitatively similar to (4.5.9). For estuaries of interest to this chapter, however, it is clear that the dependence of $c_{g}$ on low friction makes its use physically inappropriate.

More recently, shorter-rising tides in frictionally-dominated channels have been attributed in large part to tidal modulation of depth in the friction term of the momentum equation (e.g., Parker 1984; Friedrichs and Aubrey 1988). The results presented in this section, which are well-supported by observations, also refute this explanation. Instead, it is suggested that non-linear friction plays a less important role in generating tidal asymmetry in the natural tidal channels of interest to this chapter. The effects described by (4.5.9) are entirely due to non-linear continuity.

\subsection{Discussion of second-order solution for velocity}

\subsubsection{Dominant velocity frequency}

Like the second-order solution for $\eta_{1}$, the second-order solution for $V_{1}$ (4.4.23) describes a purely progressive wave, with the same phase speed as the first-order wave, and with an exponentially-modulated amplitude. An important feature of (4.4.23) is the role played by $\varepsilon_{T}$ in determining the phase of $V_{1}$ relative to $\eta_{1}$. Maintaining accuracy to $\mathrm{O}\left(\varepsilon_{\Gamma}\right),(4.4 .23)$ may be rewritten as

$$
V_{1}=-\mathrm{e}^{\mu k x}\left\{\cos \varepsilon_{T} \sin (\omega t-k x)-\sin \varepsilon_{T} \cos (\omega t-k x)\right\},
$$

or simply,

$$
V_{1}=-\mathrm{e}^{\mu k x} \sin \left(\omega t-k x-\varepsilon_{T}\right)
$$

Thus to $O(\varepsilon T), V_{1}$ leads $\eta_{1}$ by

$$
\varphi_{\eta 1}-\varphi_{V 1}=\frac{\pi}{2}-\varepsilon_{T}
$$

If $\varepsilon_{T}$ is vanishingly small, i.e., if along-channel convergence is infinitely strong, then $V_{1}$ leads $\eta_{1}$ hy $90^{\circ}$, which is identical to the first-order case. For larger $\varepsilon_{T}, V_{1}$ leads $\eta_{1}$ by a smaller phase.

Using (4.2.8b) and (4.3.16), (4.6.3) may be re-expressed as

$$
\varphi_{\eta 1}-\varphi_{V 1}=\frac{\pi}{2}-\frac{8}{3 \pi} \frac{c_{d} \varepsilon_{h} \omega^{2} L_{A}^{3}}{g(\bar{A} / \bar{b})^{2}} .
$$


Equation (4.6.4) indicates that in a strongly convergent tidal channel near morphologic equilibrium, $\varphi_{\eta 1}-\varphi_{V 1}$ responds primarily to local geometric conditions. This result is in sharp contrast to the classical view of frictionally damped co-oscillating tides in channels. Classical theory indicates that the relative phase of velocity should be a strong function of $x / L$. For a frictionally damped co-oscillating tide in a prismatic channel, $\varphi_{\eta 1}-\varphi_{V 1} \rightarrow 90^{\circ}$ as $x \rightarrow L$ because of complete reflection at the head, and $\varphi_{\eta 1}-\varphi_{V 1}$ decreases as $x \rightarrow 0$ as the reflected wave becomes more damped with respect to the incident wave.

Figure 4.7 displays observations of $\varphi_{\eta 1}-\varphi_{V 1}$ from the Delaware (Parker 1984), Thames (Hunt 1964) and Tamar (Uncles and Stephens 1990) as a function of $\varepsilon_{T}$, superimposed on (4.6.3). All the velocity time-series available for the Delaware and Thames are point measurements and, beyond testing $\varphi_{\eta 1}-\varphi_{V 1}$, are of limited use for examining the 1-D theory developed in this chapter. The velocity record for the Delaware is from $x / L=0.39$ (Parker 1984), which is in the second "segment" of the estuary (see Table 4.4). Thus the calculation of $\varepsilon_{T}$ used here is based on the geometry of that segment. The three velocity records for the Thames are from $x / L=0,0.38$ and 0.73 (Hunt 1964), all of which are in the first segment of the Thames, and $\varepsilon_{T}$ for the Thames is likewise defined. The agreement between observations and (4.6.3) is quite good for the Delaware, the Tamar and for two of the three observations from the Thames. The poorly matched point is for observations at $x / L=0.73$ in the Thames, which is relatively near the transition to segment 2. The data point in parentheses is $\varphi_{\eta 1}-\varphi_{V 1}$ for the velocity record at $x / L=0.73$ plotted versus the $\varepsilon_{T}$ value appropriate to segment 2 .

In (4.6.2), the parameter $\mu$ determines the growth or decay of $\left|V_{1}\right|$ as a function of distance along channel. It was shown in Chapter 3 that in stable tidal channels, the amplitude of tidal velocity is nearly constant along-channel. Figure 4.3 indicates this is the case along the Delaware, Thames and Tamar. Thus tides in channels near morphologic equilibrium should, by definition, have $\mu \approx 0$. The theory developed in this Chapter is therefore closely linked to the morphodynamic evolution of tidal channels. If in an exponentially-shaped tidal estuary, $\mu$ is significantly less than or greater than zero, then the system may not be near morphologic equilibrium. Significant gradients in along-channel tidal velocity should cause along-channel gradients in sedimentation or erosion, leading to the eventual adjustment of $\mu$ toward zero. Of course there are important limitations to this conclusion. Primary among them is the contribution of tidal harmonics toward spatial gradients in peak velocity and toward asymmetries in the direction of peak velocity. Likely 
relationships between temporal asymmetries and spatial gradients in velocity in stable channels are discussed further in Chapter 3.

\subsubsection{Second harmonic}

From (4.4.24) and (4.6.3), the amplitude ratio of $V_{2}$ to $V_{1}$ is

$$
\left|\frac{V_{2}}{V_{1}}\right|=\frac{|\gamma|}{2}\left|1-2 \mathrm{e}^{\mu k x} \mathrm{e}^{-i k x}\right|=\frac{|\gamma|}{2}\left(1-4 \mathrm{e}^{\mu k x} \cos k x+4 \mathrm{e}^{2 \mu k x}\right)^{1 / 2} .
$$

$\left|V_{2} / V_{1}\right|$, like $\left|\eta_{2} / \eta_{1}\right|$, is independent of $x_{0}$. But unlike $\left|\eta_{2} / \eta_{1}\right|,\left|V_{2} / V_{1}\right|$ is predicted to be nonzero at $x=0$. For $\mu=0,\left|V_{2} / V_{1}\right|$ is simply .

$$
\left|\frac{V_{2}}{V_{1}}\right|=\frac{|\gamma|}{2}\{5-4 \cos k x\}^{1 / 2} \text {. }
$$

Time series of cross-sectionally averaged velocity are available only for the Tamar (Uncles et al. 1985). Figure 4.8 compares (4.6.5) and (4.6.6) to the results of harmonic analyses of spring velocity observations. $\gamma=0.75$ is again used, based on the mean "dynamic" tidal amplitude. Equation (4.6.6) reproduces the same order of $\left|V_{2} / V_{1}\right|$ as that observed in the Tamar. However there is no discernable along-channel trend in the observed data. Nonetheless, these results are encouraging given that velocity in general is much more sensitive than tidal elevation to measurement location and to variations in channel shape away from an idealized geometry.

From (4.6.24) and (4.6.2), the phase of $V_{2}$ relative to $V_{1}$ with $\mu=0$ can be shown to be (see Appendix 3)

$$
\begin{aligned}
& 2 \varphi_{V 1}-\varphi_{V 2}= \\
& 2 k x+2 \varepsilon_{T}-\arctan \left\{\frac{2 \sin \left(2 k x+k x_{0}\right)-\sin \left(k x+k x_{0}\right)}{2 \cos \left(2 k x+k x_{0}\right)-\cos \left(k x+k x_{0}\right)}\right\}+\frac{\pi}{2} \frac{\gamma}{|\gamma|} .
\end{aligned}
$$

Note that $2 \varphi_{V 1}-\varphi_{V 2}$, like $2 \varphi_{\eta 1}-\varphi_{\eta 2}$, is a function of $x_{0}$.

If $k x \ll 1$ (which is true for the Tamar), then $\sin k x \approx k x, \cos k x \approx 1$ and $\arctan k x$ $\approx k x$, and (4.6.7) reduces directly to

$$
2 \varphi_{V 1}-\varphi_{V 2}=\frac{\pi}{2} \frac{|\gamma|}{\gamma}+2 \varepsilon_{T}-k x-k x_{0} .
$$

Or equivalently, 


$$
2 \varphi_{V 1}-\varphi_{V 2}=\frac{\pi}{2} \frac{|\gamma|}{\gamma}+k\left(2 L_{A}-x-x_{0}\right)
$$

Figure 4.9 displays $2 \varphi_{V 1}-\varphi_{V 2}$ as determined from velocity observations from the Tamar (Uncles et al. 1985) along with (4.6.9) for both $x_{0}=0$ and $x_{0}=L / 2$. Equation (4.6.9) does a reasonable job of reproducing both the magnitude and the trend of along-channel variation in $2 \varphi_{V 1}-\varphi_{V 2}$ observed for the Tamar. More importantly, (4.6.9) captures the varying nature of velocity asymmetry along the Tamar.

The relative phase of $V_{2}$ is a primary indicator of whether tidal velocity is flooddominated $\left(-90^{\circ}<2 \varphi_{V 1}-\varphi_{V 2}<90^{\circ}\right)$ or ebb-dominated $\left(90^{\circ}<2 \varphi_{V 1}-\varphi_{V 2}<270^{\circ}\right)$ (Aubrey and Speer 1985). For short channels with $\gamma>0$, (4.6.12) indicates that velocity will be ebb-dominated for $x<2 L_{A}-x_{0}$ and will be flood-dominated for $x>2 L_{A}-x_{0}$. If $\gamma<0$, (4.6.9) predicts the opposite will be true. The predicted switch in dominance (with $\gamma>0$ ) is consistent with observations taken along the Tamar (Figure 4.9). A similar alongchannel transition from ebb- to flood-dominance has also been observed in tidal channels at Murrells Inlet, South Carolina, and at Chatham Inlet in Massachusetts, and in 1-D numerical modeling of frictionally-dominated tidal channels (Friedrichs et al. 1992).

\subsection{Summary and conclusions}

A scaling of the continuity equation appropriate to strongly convergent channels (such as the Delaware in the U.S. and the Thames and Tamar in the U.K.) indicates gradients in tidal discharge are dominated at first-order by gradients in cross-sectional area. Finite amplitude effects and gradients in velocity due to tidal phase enter at second-order. Gradients in the amplitude of tidal velocity enter only at third order -- a property attributed to the channels being near morphologic equilibrium. A scaling of the momentum equation indicates the first-order balance to be between pressure gradient and friction. Local acceleration enters at second-order, and advective acceleration enters only at fourth order.

The first-order governing equation for elevation is a first-order wave equation, in contrast to the classic second-order equation which results from low friction and a prismatic channel. Assuming cross-sectional area to vary exponentially along-channel, the first-order solutions for both elevation and velocity are constant amplitude, forward-propagating wave forms with velocity leading elevation by $90^{\circ}$. The form of the first-order solution diverges from the conventional view of co-oscillating tides. Velocity and elevation have the same phase relation as a standing wave, yet they are individually progressive. Furthermore, the 
solutions are entirely independent of the length of the estuary -- in sharp contrast to the length-sensitive quarter-wave resonance of classic tidal estuary theory.

First-order solutions for tidal wave speed and tidal velocity are given by $c=$ $g(\bar{A} / \bar{b})\left(r L_{A}\right)^{-1}$ and $U=a \omega L_{A}(\bar{A} / \bar{b})^{-1}$, where $\bar{A} / \bar{b}$ is the time-averaged ratio of channel crosssectional area divided by total embayment width (including tidal flats), $a$ and $\omega$ are tidal amplitude and frequency, $L_{A}$ is the along-channel e-folding length of cross-sectional area, and $r$ is the linear friction factor. Solutions for channels of similar dimensions to the Delaware, Thames and Tamar give tidal velocities and wave lengths (predicted via $c$ ) which are consistent with observations.

The second-order governing equation is also a first-order wave equation, but includes the effects of finite amplitude and phase-generated velocity gradients from continuity and the effects of local acceleration from momentum. Second-order effects arising from friction are not included because previous observations have not demonstrated that quadratic drag with tidal modulation of depth-dependence accurately reproduces observed nonlinearities in natural tidal channels. Finite amplitude effects in the continuity equation are treated by a formal perturbation in time only, with spatial effects treated kinematically. This simplifies the form of the solution without greatly affecting its accuracy for relatively short systems.

Second-order solutions for elevation and velocity at the dominant frequency are purely progressive wave-forms with the same phase speed as the first-order solution. At second order the phase lead of velocity is predicted to decrease away from $90^{\circ}$ as $k L_{A}$ increases, where $k$ is the first-order wave-number. Amplitude of both elevation and velocity are modulated by $\mathrm{e}^{\mu k x}$, where $\mu=\omega / r-k L_{A}$. $\mu$ represents the partially cancelling effects of local acceleration and limited convergence. Because $\mu$ determines the growth or decay of tidal velocity with distance along channel, we expect to find $\mu \approx 0$ in channels near morphologic equilibrium. The quantity $\omega / r-k L_{A}$ is close to zero along the Delaware, Thames and Tamar.

Observations of small scale variations in tidal amplitude and phase along the Delaware and Thames are reproduced by fitting exponential geometries to individual channels segments and applying the second-order solution to each segment. This is easily done for the unidirectional wave solution. The only boundary condition on each segment is the amplitude and phase at the end of the previous segment. The segmented solution demonstrates that along-channel undulations in amplitude and phase are due primarily to 
changes in the rate of convergence of channel geometry and are not due primarily to interactions between individual incident and reflected waves as predicted by classical cooscillation.

Compact solutions for tidal elevation at the second harmonic frequency are consistent with observations from the Delaware, Thames and Tamar. The second harmonic is scaled by $\gamma=a / \bar{h}-\Delta b / \bar{h}$, where $\bar{h}$ is channel depth and $\Delta b$ is the amplitude of tidal variation in system width. Control of elevation asymmetry by $\gamma$ is described conceptually by replacing $\bar{A} / \bar{h}$ in $c$ with $A(t) / b(t)$. Then if $\gamma>0$, the wave crest propagates faster than the trough, causing the rising tide to be of shorter duration. If $\gamma<0$, the opposite holds and the falling tide is shorter. $\gamma$ is greater than zero for the Delaware, Thames and Tamar. Solutions for the zeroth harmonic are also scaled by $\gamma$.

Compact solutions for sectionally-averaged tidal velocity at the second harmonic frequency are also reasonably consistent with observations (which are available only for the Tamar). Like elevation, the second harmonic of velocity is also scaled by $\gamma$. Unlike elevation, however, the sense of velocity asymmetry is predicted to reverse a short distance into the channel. For $\gamma>0$, ebb currents dominate at the mouth, whereas at distances beyond about $2 L_{A}$, flood currents dominate. For $\gamma<0$, the opposite pattern is predicted, with flood currents dominating at the mouth and ebb currents dominating within. 
Table 4.1. Observed and computed tidal and geometric properties of three tidal estuaries. Parameters are further defined in the text. \pm indicates standard errors; $<>$ indicates along-channel average.

\begin{tabular}{|c|c|c|c|}
\hline Parameter & Delaware & Thames & Tamar \\
\hline$L(\mathrm{~km})$ & 215 & 95 & 21 \\
\hline$L_{T}(\mathrm{~km})$ & $370 \pm 10$ & $450 \pm 80$ & $270 \pm 10$ \\
\hline$<U>(\mathrm{m} / \mathrm{s})$ & $0.63 \pm 0.02$ & $0.63 \pm 0.05$ & $0.53 \pm 0.01$ \\
\hline$<a>(\mathrm{m})$ & $0.83 \pm 0.03$ & $2.2 \pm 0.1$ & $2.3 \pm 0.1$ \\
\hline$<\bar{h}>(\mathrm{m})$ & $5.8 \pm 0.3$ & $7.2 \pm 0.6$ & $2.5 \pm 0.2$ \\
\hline$\left\langle\varepsilon_{h}\right\rangle=\frac{\langle a\rangle}{\langle\bar{h}\rangle}$ & $0.14 \pm 0.01$ & $0.31 \pm 0.03$ & $0.92 \pm 0.08$ \\
\hline$\left\langle\varepsilon_{b}\right\rangle=\frac{\langle\bar{b}-w\rangle}{\langle\bar{b}\rangle}$ & $\sim 0$ & $0.20 \pm 0.02$ & $0.30 \pm 0.09$ \\
\hline$L_{b}(\mathrm{~km})$ & $34 \pm 1$ & $23 \pm 5$ & $4.6 \pm 0.4$ \\
\hline$L_{A}(\mathrm{~km})$ & $32 \pm 1$ & $19 \pm 1$ & $5.3 \pm 0.3$ \\
\hline$L_{A} / L_{U}$ & $0.012 \pm 0.013$ & $0.069 \pm 0.075$ & $0.033 \pm 0.013$ \\
\hline$\varepsilon_{U}=\left(L_{A} / L_{U}\right)^{1 / 2}$ & $0.11 \pm 0.08$ & $0.27 \pm 0.22$ & $0.18 \pm 0.05$ \\
\hline$\varepsilon_{T}=2 \pi L_{A} / L_{T}$ & $0.56 \pm 0.02$ & $0.27 \pm 0.05$ & $0.12 \pm 0.01$ \\
\hline$<\bar{A} / \bar{b}>(\mathrm{m})$ & $5.8 \pm 0.3$ & $7.0 \pm 0.5$ & $1.7 \pm 0.2$ \\
\hline$\frac{\langle a\rangle \omega L_{A}}{\langle\bar{A} / \bar{b}\rangle\langle U\rangle}$ & $1.0 \pm 0.1$ & $1.3 \pm 0.2$ & $1.9 \pm 0.2$ \\
\hline$\varepsilon_{a}=\left(L_{A} / L_{a}\right)^{1 / 2}$ & $0.24 \pm 0.03$ & $0.11 \pm 0.21$ & $0.27 \pm 0.04$ \\
\hline$\langle w / \bar{b}\rangle$ & $\sim 1$ & $0.80 \pm 0.02$ & $0.70 \pm 0.09$ \\
\hline$\frac{\varepsilon_{T} c^{2}}{\langle w / \bar{b}>g<\bar{h}\rangle}$ & $0.65 \pm 0.04$ & $0.47 \pm 0.09$ & $0.26 \pm 0.04$ \\
\hline
\end{tabular}


Table 4.2. Small terms used in scaling governing equations and in approximating solution.

\begin{tabular}{|c|c|} 
Formally second-order, $O(\varepsilon)$ quantities & Other informally small quanitities \\
\hline$\varepsilon_{h}=a / \bar{h}$ & $h / w$ \\
$\varepsilon_{b}=\Delta b / \bar{b}$ & $\lambda=L_{/}-L / L_{b}$ \\
$\varepsilon_{U}=\left(L_{A} / L_{U}\right)^{1 / 2}$ & $L_{A} / L(1)$ \\
$\varepsilon_{T}=2 \pi L_{A} / L_{T}$ & $L_{L}\left(L_{T}{ }^{(2)}\right.$ \\
$\varepsilon_{a}=\left(L_{A} / L_{a}\right)^{1 / 2}$ & \\
$\varepsilon_{\omega}=\omega / r$ & \\
$\mu=\varepsilon_{\omega}-\varepsilon_{T}$ & \\
$\gamma=\varepsilon_{h}-\varepsilon_{b}$ &
\end{tabular}

(1)Important in vicinity of landward boundary. (2)Important for second-harmonic solution. 
Table 4.3. See Table 4.1 for explanation.

\begin{tabular}{|c|c|c|c|} 
Parameter & Delaware & Thames & Tamar \\
\hline$\lambda=L / L_{A}-L / L_{b}$ & $0.30 \pm 0.17$ & $0.48 \pm 0.29$ & $-0.60 \pm 0.20$ \\
\hline $\begin{array}{c}U=\frac{<a>\omega L_{A}}{<\bar{A} / \bar{b}>} \\
(\mathrm{m} / \mathrm{s})\end{array}$ & $0.65 \pm 0.05$ & $0.83 \pm 0.08$ & $1.00 \pm 0.11$ \\
\hline$c_{d}=\frac{3 \pi^{2} g(\bar{A} / \bar{b})^{2}}{4 L_{T}<\varepsilon_{h}>\omega^{2} L_{A}{ }^{2}}$ & $2.3 \pm 0.2 \times 10^{-3}$ & $3.7 \pm 0.8 \times 10^{-3}$ & $1.6 \pm 0.3 \times 10^{-3}$ \\
\hline $\begin{array}{c}L_{g}=\frac{2 \pi}{\omega}(g<\bar{h}>)^{1 / 2} \\
(\mathrm{~km})\end{array}$ & $340 \pm 10$ & $380 \pm 20$ & $220 \pm 10$ \\
\hline$<\gamma=<\varepsilon_{h}>-<\varepsilon_{b}>$ & $0.14 \pm 0.01$ & $0.10 \pm 0.04$ & $0.63 \pm 0.12$ \\
\hline$\varepsilon_{\omega}=\frac{3 \pi<\bar{A} / \bar{b}>}{8 c_{d}<\varepsilon_{h}>L_{A}}$ & $0.65 \pm 0.09$ & $0.39 \pm 0.10$ & $0.27 \pm 0.06$ \\
\hline$\mu=\varepsilon_{\omega}-\varepsilon_{T}$ & $0.094 \pm 0.091$ & $0.12 \pm 0.11$ & $0.15 \pm 0.06$ \\
\hline$\mu=\frac{1}{2 \pi} \frac{L_{T}}{L_{a}}$ & $0.061 \pm 0.009$ & $-0.06 \pm 0.12$ & $-0.58 \pm 0.05$ \\
\hline
\end{tabular}

*If $L_{a}$ is based on the "dynamic" tidal amplitude, defined as local high water minus mean tide level at $x=0$. 
Table 4.4. See Table 4.1 for explanation.

\begin{tabular}{c|c|c|c|c|c|}
\multicolumn{1}{c}{ Estuary } & \multicolumn{1}{c}{ Segment } & $x / L$ & $\mu$ & $L_{T}(\mathrm{~km})$ & $L_{A}(\mathrm{~km})$ \\
\hline \multirow{2}{*}{ Thames } & 1 & $0-0.8$ & 0.34 & 630 & 22 \\
& 2 & $0.8-1$ & -0.41 & 130 & 13 \\
\hline \multirow{2}{*}{ Delạware } & 1 & $0-0.22$ & 0.54 & 530 & 40 \\
& 2 & $0.22-0.68$ & -0.10 & 310 & 44 \\
& 3 & $0.68-1$ & 0.37 & 450 & 33 \\
\hline
\end{tabular}


Figure captions for Chapter 4

Figure 4.1. Diagram of an idealized tidal embayment cross-section: $b$ is embayment width (including storage in tidal flats), $\zeta$ is tidal elevation, $h$ is crosssectionally averaged channel depth, $w$ is channel width (which is equal to embayment width at low tide), and $A$ is channel cross-sectional area. Over-bars indicate time averages.

Figure 4.2. Observed semi-diumal surface tide as a function of distance along the Delaware (Parker 1984), Thames (Prandle 1980) and Tamar (George 1975): (a) amplitude, and (b) phase, along with least-squares linear regressions.

Figure 4.3. Observations of channel cross-sectional area at mid-tide, timeaveraged embayment width, and cross-sectionally averaged velocity amplitude as a function of distance along the (a) Delaware (Parker 1984), (b) Thames (Hunt 1964), and (c) Tamar (Uncles et al. 1985), along with least-squares log-linear regressions.

Figure 4.4. Observed semi-diurnal surface tide as a function of distance along the Tamar (George 1975), Delaware (Parker 1984), and Thames (Prandle 1980) along with predictions given by second-order solutions: (a) amplitude, (b) phase. Observations of amplitude along the Tamar are calculated as the difference between local high water elevation minus mid-tide elevation at $x=0$. Predictions for the Delaware and Thames are based on the "segmented" solution.

Figure 4.5. Observed relative amplitudes for the second harmonic of tidal elevation as a function of distance along (a) the Delaware (Parker 1984), and (b) the Tamar (George 1975) and Thames (Prandle 1980), along with predictions given by the secondorder solution.

Figure 4.6. Observed relative phases for the second harmonic of tidal elevation as a function of distance along (a) the Delaware (Parker 1984), and (b) the Tamar (George 1975) and Thames (Hunt 1963), along with predictions given by the second-order solution.

Figure 4.7. Observed phase of velocity relative to elevation at the dominant tidal frequency for the Delaware (Parker 1984), Thames (Hunt 1963) and Tamar (Uncles and Stephens 1990), along with predictions given by the second-order "segmented" solution as a function of $\varepsilon_{T}=2 \pi L_{T} / L_{A}$. The poorly matched point for the Thames is in segment 
one, but relatively near the transition to segment two. Parentheses indicate the same paint plotted using the geometry of segment two.

Figure 4.8. Observed relative amplitudes for the second harmonic of tidal velocity as a function of distance along the Tamar (Uncles et al. 1985), along with predictions given by the second-order solution.

Figure 4.9. Observed relative phases for the second harmonic of tidal velocity as a function of distance along the Tamar (Uncles et al. 1985), along with predictions given by the second-order solution. 


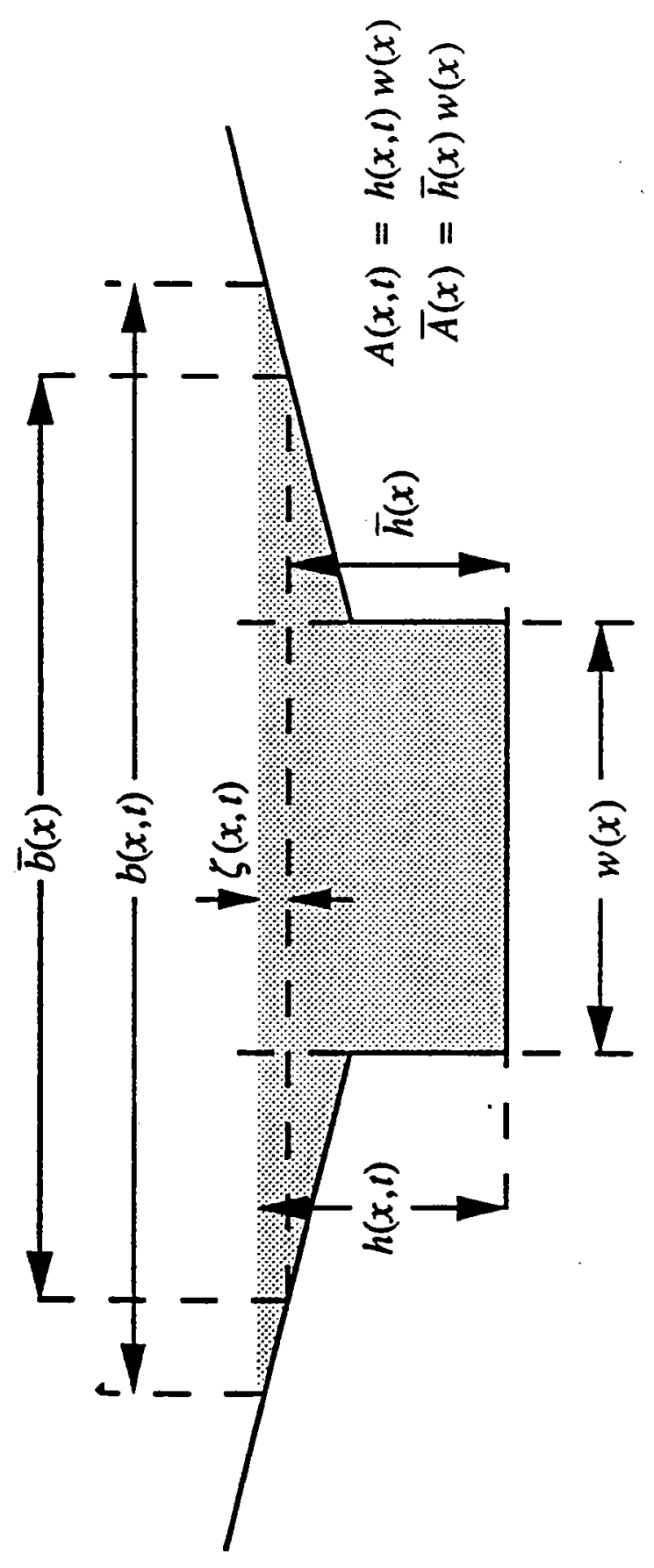

Figure 4.1 

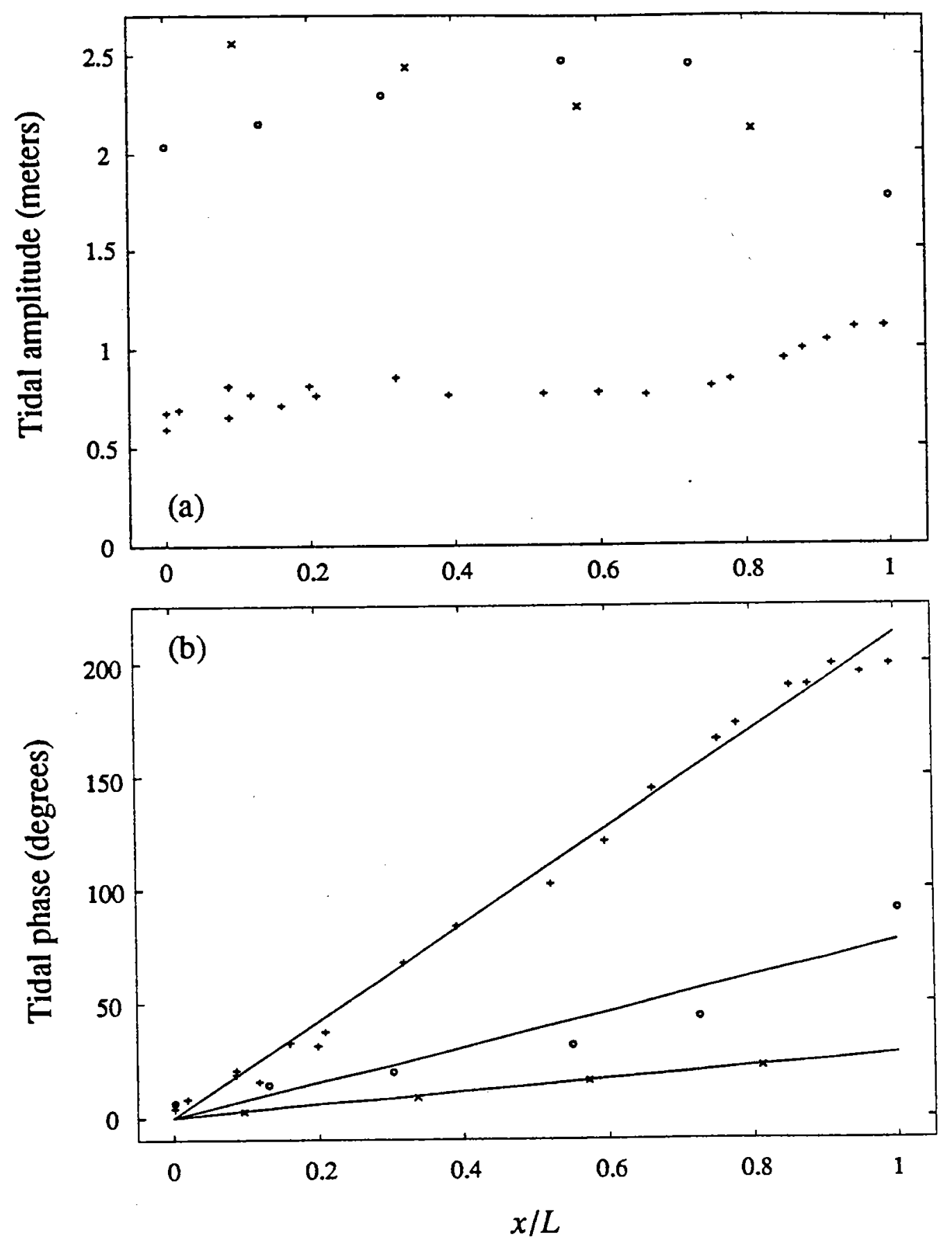

+ Delaware $\quad 0=$ Thames $\quad x=$ Tamar

Least-squares fit to (4.2.3a)

Figure 4.2 

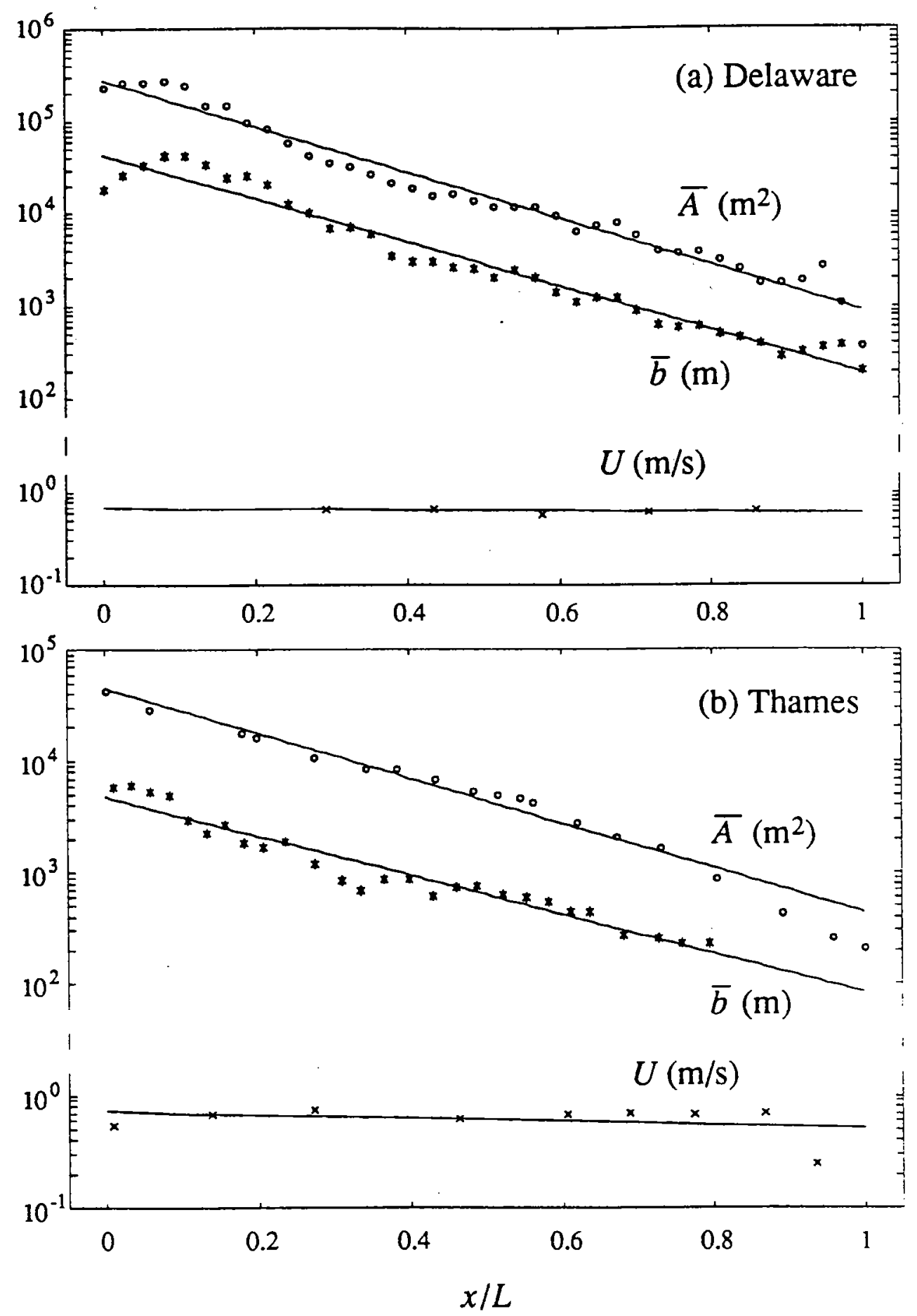

Least-squares fit to (4.2.6)

Figure 4.3 


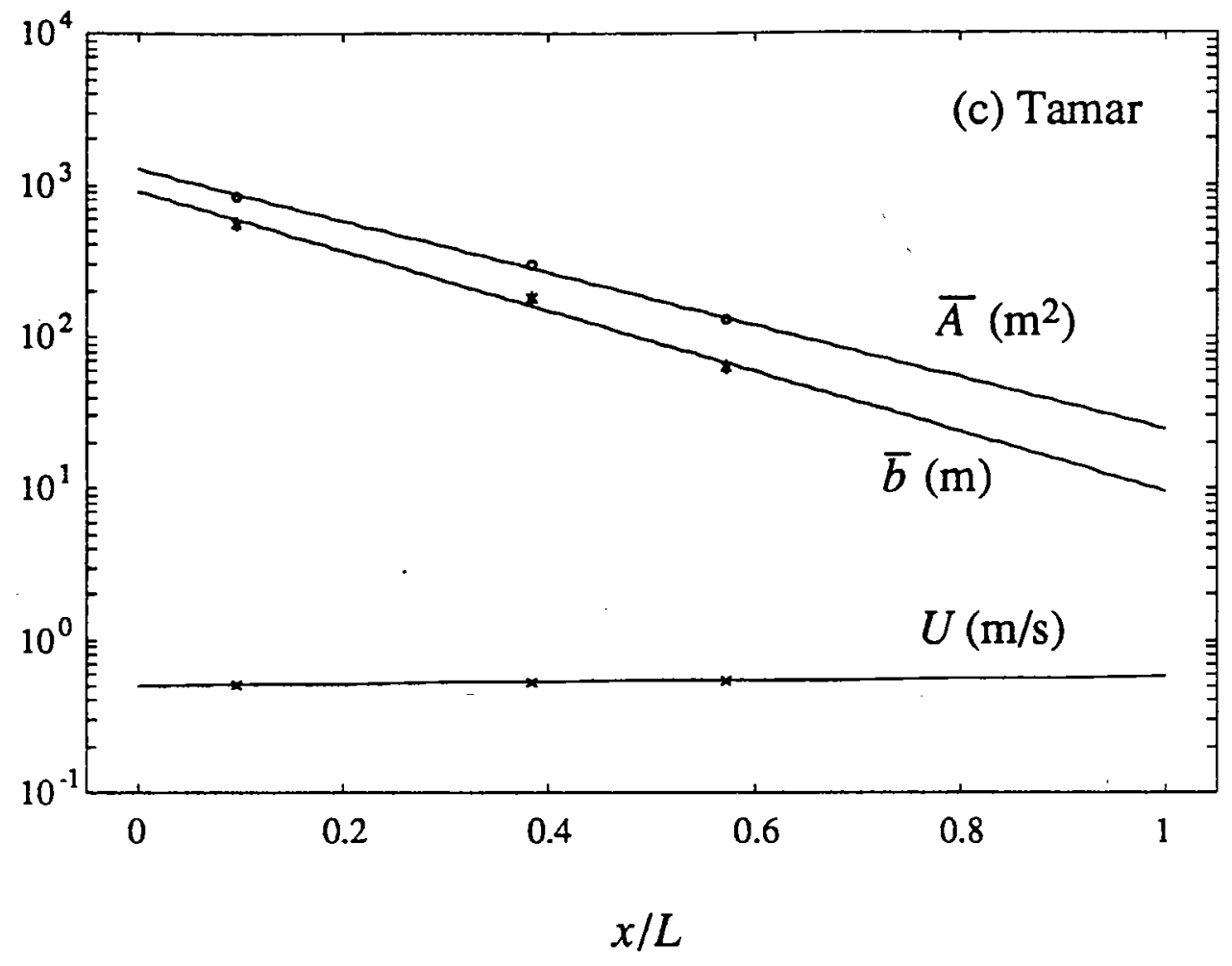

Least-squares fit to (4.2.6)

Figure 4.3 (cont.) 

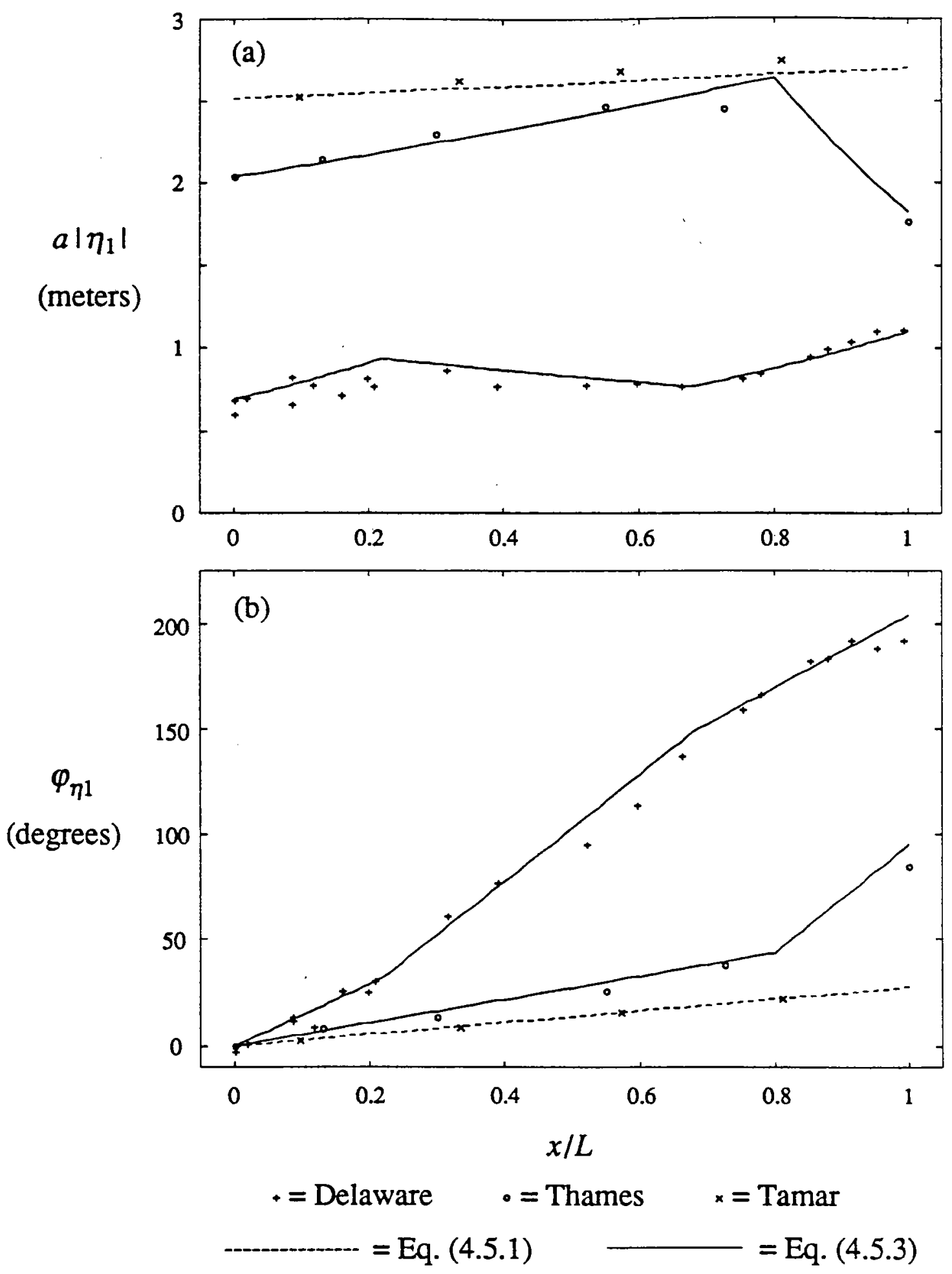

Figure 4.4 

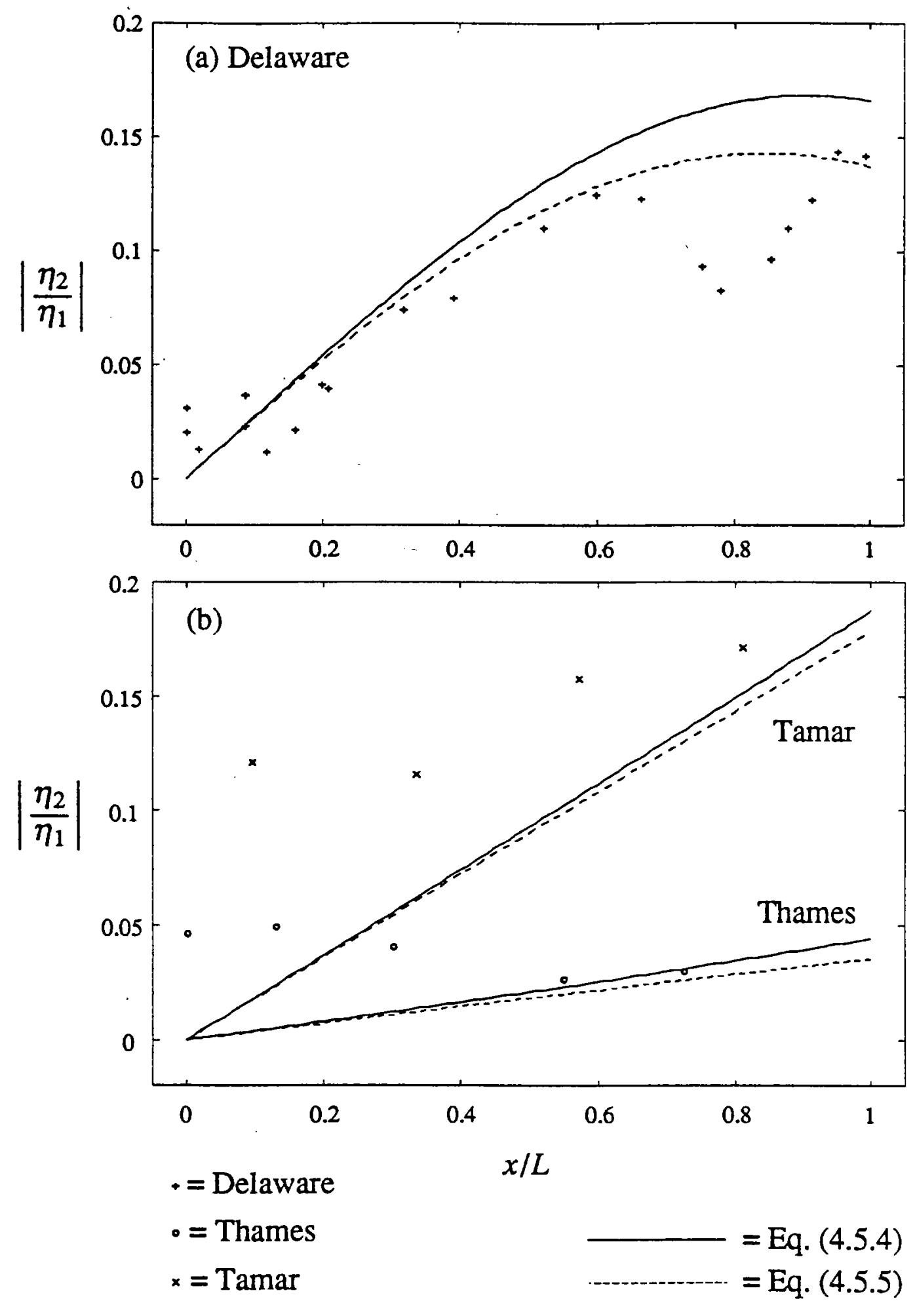

Figure 4.5 

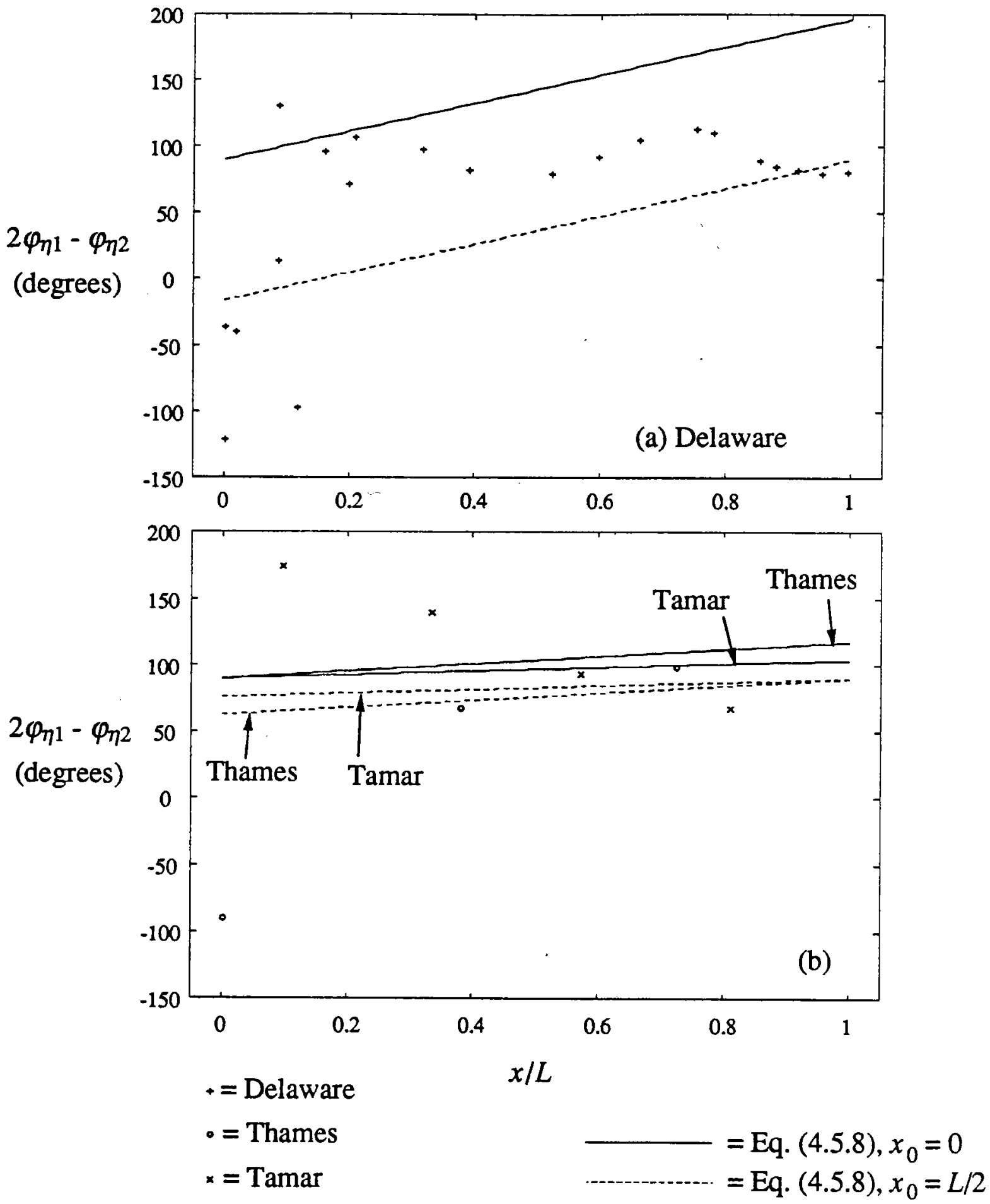

Figure 4.6 


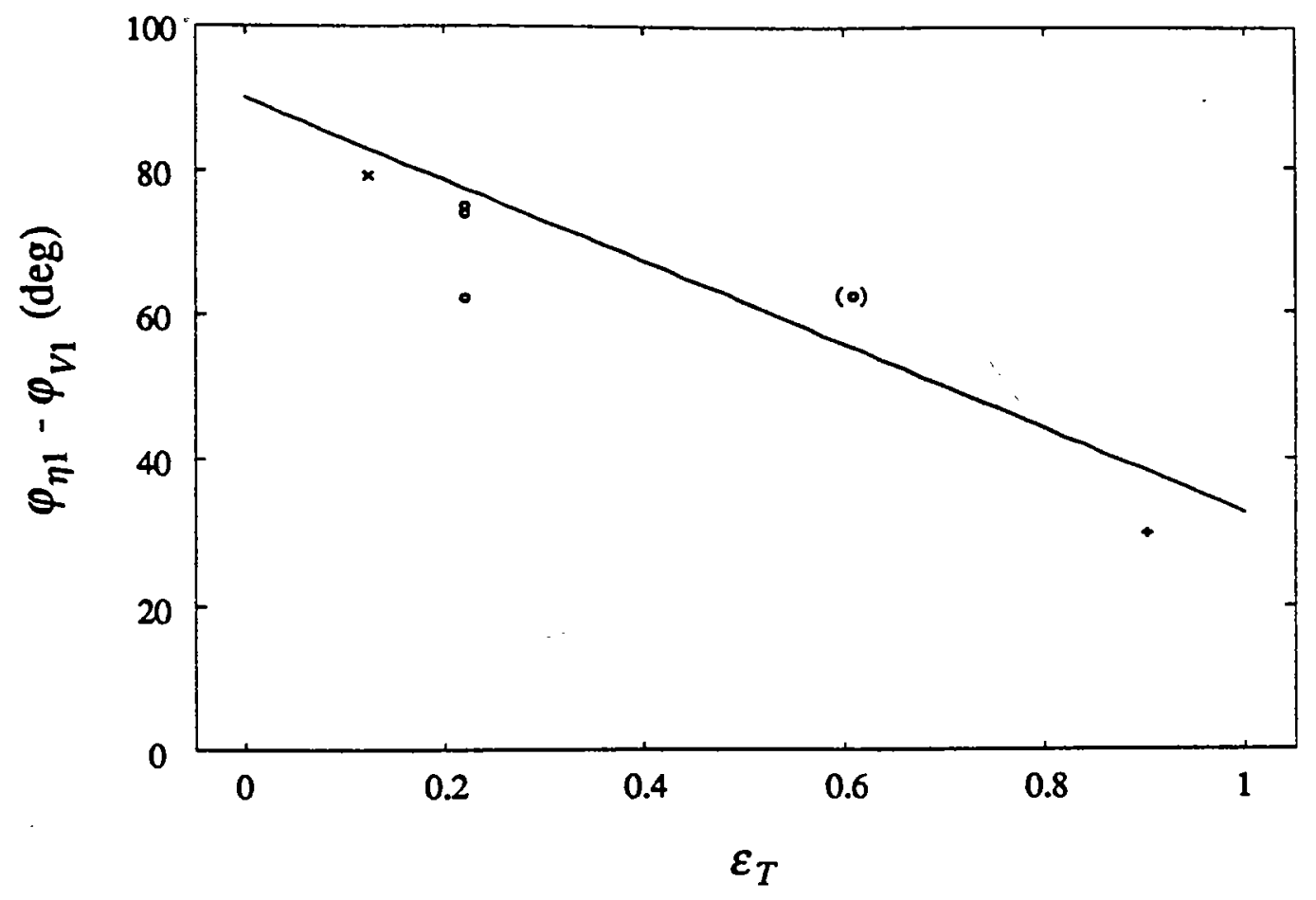

$$
\begin{aligned}
& +=\text { Delaware } \\
& \bullet=\text { Thames } \quad-\quad=\text { Eq. }(4 \cdot 6 \cdot 3) \\
& x=\text { Tamar }
\end{aligned}
$$

Figure 4.7 

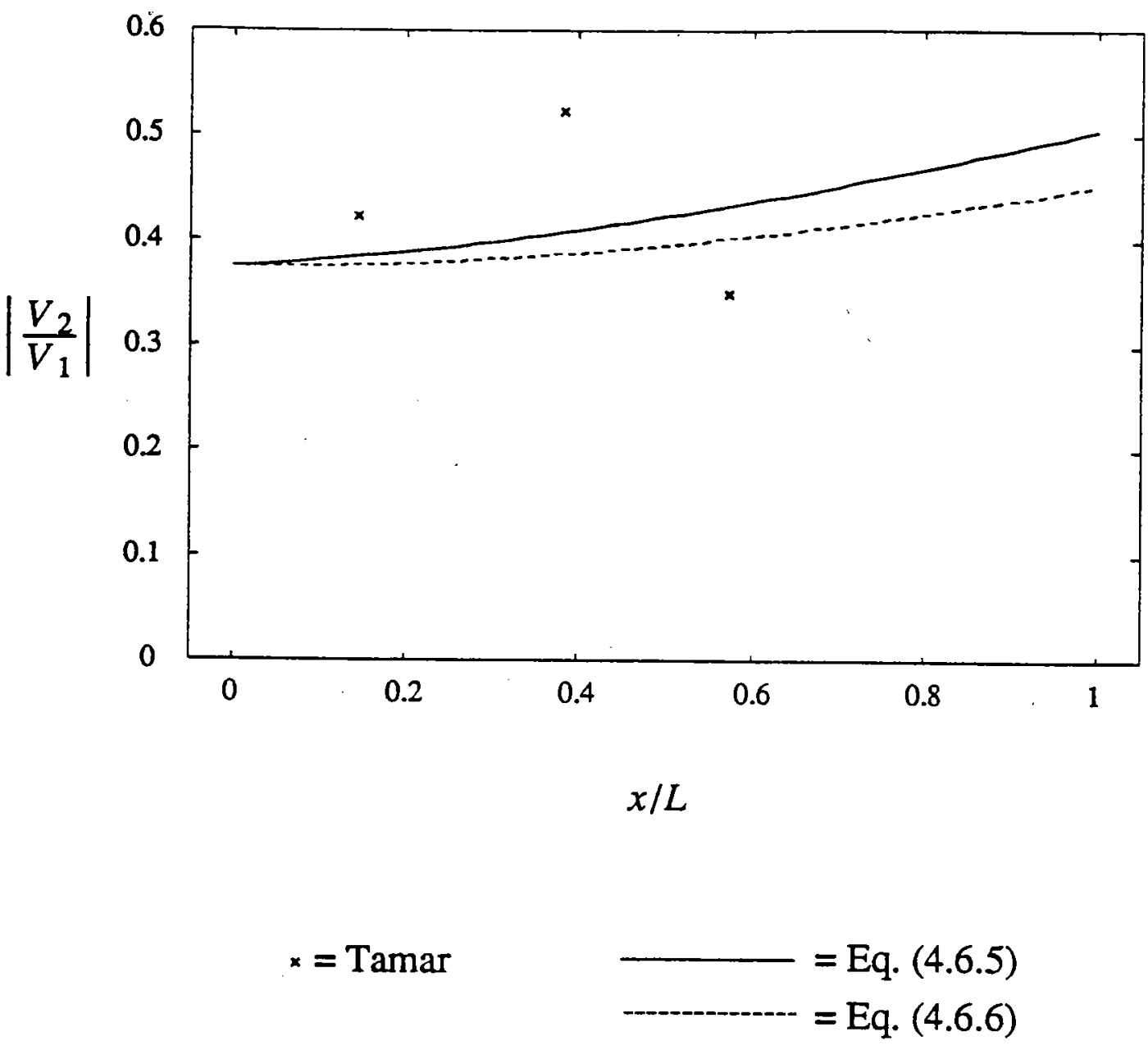

Figure 4.8 


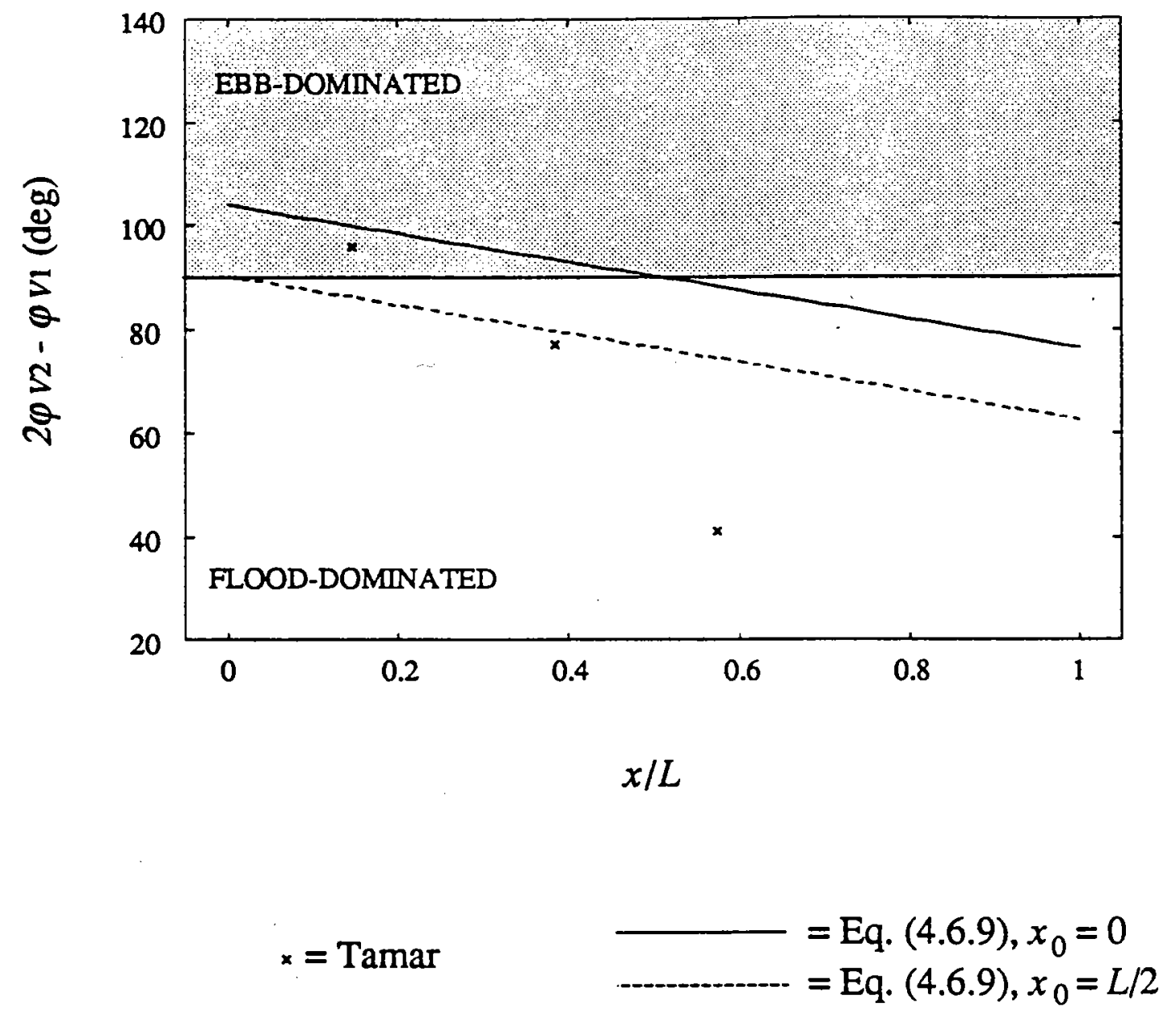

Figure 4.9 
-150 -

Chapter 5:

Equilibrium Hypsometry of Intertidal Flats 


\section{Abstract to Chapter 5}

Recent observations of tidal flat morphology have correlated convex hypsometry with large tide ranges, long-term accretion and/or low wave activity. Concave hypsometry, in turn, has been correlated with small tide ranges, long-term erosion and/or high wave activity. This study demonstrates that much of this empirically observed variation in tidal flat hypsometry may be explained by a simple morphodynamic model which assumes tidal flats to be at equilibrium if maximum bottom shear stress $(\tau)$ is spatially uniform. Two general cases are considered: (i) absence of wind waves where $\tau$ is equal to maximum tidal shear stress, and (ii) dominance by wind waves, where $\tau$ is equal to maximum wavegenerated shear stress.

If one assumes a spatially uniform drag coefficient at the time of maximum shear stress, then uniform maximum velocity may be used as a proxy for uniform $\tau$. Conservation of mass is used to determine the distribution of maximum tidal velocity $\left(U_{T}\right)$ across the flat, whereas conservation of energy determines the distribution of maximum wave orbital velocity $\left(U_{W}\right)$. Results indicate that a flat which slopes linearly away from a straight shoreline does not produce a uniform distribution of $U_{T}$ or $U_{W}$, and therefore is not at equilibrium under domination by either.

If the profile is adjusted until $U_{T}$ is constant, then $U_{T}$ is found to be proportional to the length of the tidal flat, $L$, and the equilibrium profile is predicted to be convex. The equilibrium profile for $U_{W}$, in contrast, is concave and has depth increasing like $x^{2 / 3}$, a form which has been reported empirically for dissipative beaches. Under domination by $U_{W}, L \sim h_{0}{ }^{2} / H_{0}$, where $h_{0}$ is the tidal range and $H_{0}$ is the forcing wave height. This expression indicates that $L$ should increase dramatically with tidal range. Since $U_{T} \sim L$, the relative importance of tidal currents should also increase strongly with tidal range, favoring an eventual transition from concave to convex hypsometry as tidal range increases.

Equilibrium profiles along curved shorelines are also derived by solving conservation of mass or energy. Under domination of either $U_{T}$ or $U_{W}$, results indicate that an embayed shoreline significantly enhances the convexity of the equilibrium profile, and a lobate shoreline slightly increases concavity. The nonlinear transformation from profiles to hypsometries, however, causes the hypsometry of embayed and lobate shorelines to be much more or less convex than the corresponding profiles -- so much so that the potential effect of shoreline curvature on equilibrium hypsometry is of the same order as the effect of domination by $U_{T}$ or $U_{W}$. 
List of symbols in Chapter 5

a tidal amplitude

A cumulative horizontal basin area

$A_{L} \quad A$ at $x=L$

$A_{m} \quad$ maximum value of $A$

$b \quad$ width of flat parallel to shoreline

$b_{0} \quad b$ at $x=0$

$b_{L} \quad b$ at $x=L$

$B \quad B\left(x_{1}, x_{2}\right)=\left(b\left(x_{1}\right) / b\left(x_{2}\right)+1\right) / 2$

$c_{d} \quad$ drag coefficient

$C_{1,2,3}$ constants

$C_{g} \quad$ shallow water wave group velocity

$D \quad$ energy dissipation by bottom friction

$E \quad$ wave energy density

$g$ acceleration of gravity

$h$ still water depth

$h_{0} . \quad h$ at $x=0$

$h_{m} \quad$ maximum value of $h$

$H$ wave height

$H_{0} \quad H$ at $x=0$

$\widehat{H}_{0}$ non-dimensional $H_{0}$

$L \quad$ shore-normal length of tidal flat

$L^{*} \quad$ shore-normal length of lower profile

$r$ shore-normal co-ordinate for radially symmetric flat

$r_{0} \quad r$ at $x=0$

$r_{f} \quad r$ at $x=x_{f}$

$r_{L} \quad r$ at $x=L$

$t$ time

$T_{W} \quad$ wave period

$u \quad$ velocity

$u_{e q} \quad$ equilibrium velocity

$u_{T} \quad$ depth-averaged tidal velocity

$u_{W} \quad$ wave orbital velocity

$U$ maximum depth-averaged velocity

$U_{T} \quad$ maximum depth-averaged tidal velocity 
List of symbols in Chapter 5 (continued)

$U_{T_{0}} \quad U_{T}$ at $x=0$

$U_{W}$ maximum wave orbital velocity

$U_{W_{0}} \quad U_{W}$ at $x=0$

$x \quad$ shore-normal co-ordinate

$x_{f} \quad$ postion of tidal front

$z \quad$ vertical co-ordinate

$z^{*} \quad$ elevation of transition from lower to upper profile

$Z \quad$ profile elevation

$Z_{+} \quad$ upper profile elevation

$Z$. lower profile elevation

$\eta \quad$ tidal elevation

$\theta \quad$ angle between velocity and shoreline

$\rho \quad$ fluid density

$\sigma \quad$ wave frequency

$\tau$ maximum bottom shear stress

$\tau_{\Gamma} \quad$ maximum tidal bottom shear stress

$\tau_{W}$ maximum wave-generated bottom shear stress

$\omega \quad$ tidal frequency 


\subsection{Introduction}

More than half of the world's non-arctic coastlines are either macrotidal (range > $4 \mathrm{~m}$ ) or mesotidal (range $2-4 \mathrm{~m}$ ) (Davies 1980). The study of equilibrium tidal flat morphology provides insight into the response of meso- and macrotidal coastlines to such external forcings as engineering works, periodic storm activity, and changes in relative sea level. Hypsometric curves, which measure cumulative horizontal basin area as a function of elevation, usefully represent broad aspects of tidal flat morphology in a concise and quantitative manner (e.g., Kirby 1992). Recent observations relating characteristic tidal flat hypsometries to tide range, wind wave activity, and long-term accretion or erosion provide a base of empirical data with which to compare equilibrium hypsometries predicted by analytic theory.

In this investigation equilibrium hypsometric curves are derived for intertidal flats by assuming a uniform magnitude of maximum shear stress $(\tau)$. A spatially uniform distribution of $\tau$ provides a useful first approximation to the more correct statement that a stable morphology results when there is a zero divergence in net sediment transport. The resulting analytic forms based on uniform $\tau$ are consistent with previous empirical observations and provide insight into the physical mechanisms which determine tidal flat hypsometry. Domination of $\tau$ by tidal currents is found to favor a convexity, whereas domination of $\tau$ by wind waves if found to favor concavity. The effect of shoreline curvature on equilibrium hypsometry is found to be of the same order as the effects of domination by tides or waves.

\subsubsection{Definitions and previous work}

Hypsometric analysis, which was formally introduced to geomorphology by Strahler (1952), is the study of the distribution of surface area of a land mass or basin with respect to elevation. Hypsometric curves are often presented as non-dimensional plots of relative elevation and relative surface area, allowing a comparison of hypsometric curves between systems having different scales. Strahler found distinctive hypsometric curves to be related to the erosional maturity of land regions formed in homogeneous strata. Boon (1975) and Boon and Byrne (1981) first applied hypsometric analysis to the study of intertidal basins. They used the hypsometry of intertidal storage areas to model patterns of asymmetric discharge in tidal channels near Wachapreague, Virginia. 
Figure 5.1, which is modified from Boon and Byrne (1981), shows example basin morphologies and their associated hypsometries. In Figure 5.1 and throughout this paper, hypsometric curves display the cumulative horizontal basin area below a given contour. It is important to distinguish the hypsometric curve from the topographic profile. The profile is a plot of elevation versus horizontal distance along the gradient of the topography. In Figure 5.1, for example, all three topographies have linear profiles. Along a straight shoreline (case $i$ in Figure 5.1) the profile and hypsometric curve are interchangeable. Along curved shorelines, however, the nonlinear transformation from profile to hypsometry causes a linear profile to produce a nonlinear hypsometry. If the profile is straight and the shoreline is embayed (case ii in Figure 5.1), then the hypsometry will be convex. If the profile is straight and the shoreline is lobate (case iii in Figure 5.1), then the hypsometry will be concave. Boon and Byme (1981) emphasized the sensitivity of tidal flat hypsometry to shoreline curvature.

Recent observations of tidal flat hypsometry have related the form of the hypsometric curve to other factors including tidal range, exposure to wind wave activity and patterns of long-term accretion or erosion. In a study of tidal basins along the German Bight, Dieckmann et al. (1987) noted that hypsometric curves tend to be more concave for lower tidal range flats and more convex for higher tidal range flats. In a study of macrotidal (range $>4 \mathrm{~m}$ ) flats around Great Britain, Kirby (1992) related convexity to long-term accretion and concavity to long-term erosion. At a few of the locations, Kirby in turn related accretion or erosion to protection from or exposure to wind waves. Finally, in a study of sediment exchange off the wide macrotidal flats of western Korea, Wells and Park (1992) described a periodic increase in convexity associated with a seasonal increase in wave activity.

The hypsometric trends described above can be summarized by a qualitative ratio which indicates the relative importance of tidal currents and wind waves:

$$
\begin{array}{ll}
\text { tidal/wave activity: } & \begin{array}{l}
\text { high } \rightarrow \text { CONVEX hypsometry } \\
\text { low } \rightarrow \text { CONCAVE hypsometry }
\end{array}
\end{array}
$$

This correlation is consistent with observations from the German Bight if spatial variations in tidal range are assumed to be locally more important than spatial variations in wave activity. The same trend describes flats in Great Britain if local variations in wave activity 
dominate local variations in tidal activity. The ratio describes Korean flats, too, if temporal (rather than spatial) variations in wave activity are assumed to be most important.

Potential contributors to equilibrium tidal flat hypsometry which are not addressed in this study include sediment supply and distribution of grain size. In a study of equilibrium beach profiles, Wright et al. (1985) noted an increase in concavity associated with net export of sediment from the surf zone and a decrease in concavity associated with net import. These associations are consistent with Kirby's (1992) correlation of concavity and convexity with long-term erosion and accretion on tidal flats. A gradation of sediment type from high to low water will also affect the equilibrium profile since lower equilibrium shear stresses may be associated with areas of finer grain size. Using an analytic model of shoaling waves over various shaped tidal profiles, Zimmerman (1973) suggested that a decrease in grain size toward high water enhances concavity, whereas an increase in grain size enhances convexity.

\subsubsection{Morphodynamic model}

In this study it is assumed that a stable morphology will result when the distribution of maximum bottom shear stress $(\tau)$ is uniform across a tidal flat. This is a simplification of the more correct statement that a stable morphology results when there is a zero divergence in net sediment transport. Since common formulations for erosion, deposition and net transport are generally expressed as functions of bottom shear stress -- often in the form of power relations (e.g., Dyer 1986), the spatial distribution of bottom shear stress is a useful starting point before attempting to predict sediment transport directly using more uncertain equations. Bottom shear stress can be derived from hydrodynamic relations more easily and with a greater degree of confidence.

A deviation of $\tau$ away from its mean value over a flat is assumed to cause a local increase or decrease in the rate of sediment dispersal and result in net erosion or deposition of sediment. This approach focuses on the diffusive nature of sediment transport and does not address the importance of asymmetries in the direction of bottom shear stress. The tidal and wind wave processes considered here are linearized such that no asymmetries in direction of $\tau$ are generated. Clearly, asymmetries in $\tau$ can play a morphodynamic role. For example, the morphologic impact of tidal asymmetries in embayments is the focus of Friedrichs et al. (1992) and is a primary motivation for Chapter 2 of this thesis. Nonetheless, if $\tau$ is considered to be symmetrical at first-order, then the spatial distribution 
of its magnitude alone should provide valuable insight into the morphology of stable tidal flats.

An assumption of uniform $\tau$ has also proved useful in previous investigations of equilibrium morphology. In Chapter 3 of this thesis, uniform $\tau$ was used successfully to describe the equilibrium morphology of tidal channels. Although second-order variations were associated with directional asymmetries, uniform $\tau$ provided a robust, first-order constraint on channel geometry. An assumption of uniform energy dissipation, which can be re-expressed as uniform $\tau$, has also been applied to the generation of stable beach profiles along straight shorelines (Bruun 1954). The resulting equilibrium form is consistent with empirical observations of dissipative beaches in general (Dean 1977) and has been applied successfully in individual case studies (e.g., Chappell and Eliot 1979).

For tides in the absence of wind waves or for wind waves in the absence of tides, $\tau$ has been expressed as

$$
\tau=\rho c_{d} U|U|,
$$

where $\rho$ is the fluid density, $c_{d}$ is a dimensionless drag coefficient, and $U$ is maximum depth-averaged velocity during a complete wave or tidal period. The shallow-water approximation allows the decay of wave velocity with depth to be neglected. Bottom stress given by (5.1.1) is assumed to be dominated effectively by either waves or currents. Otherwise, wave-current interaction may play a role in determining the net stress field (e.g., Grant and Madsen 1979). In this study, it is also assumed that $\rho$ and $c_{d}$ are constant in space. Under these conditions, uniform $\tau$ becomes equivalent to uniform $U$, and equilibrium morphologies can be defined by either $\tau$ or $U$.

\subsubsection{Scaling of problem: southwest coast of Korea}

Before beginning a formal derivation of equilibrium hypsometry, it is useful to scale the problem in order to assess its applicability to real tidal flats. The tidal flats along the southwest coast of Korea (Wells et al. 1990; Alexander et al. 1991; Wells and Park 1992) are chosen as a field example because of their open form and homogeneous composition, attributes which make them particularly amenable to first-order analytic modeling. Unlike many tidal flats bordering the North Sea, the Korean flats lack extensive dendritic drainage systems, seaward barriers and landward salt marshes (Alexander et al. 1991). The Korean intertidal sediments are predominantly poorly sorted mud and silt, 
whereas sediments on flats in the North Sea typically show a more well-defined shoreparallel gradation from mud to sand (Klein 1985).

Along the southwest coast of Korea the mean tide range is about six meters and tidal flats extend as much as $30 \mathrm{~km}$ out from the coast (Alexander et al. 1991; Wells and Park 1992). However a more typical shore-normal length scale is $L \approx 5 \mathrm{~km}$. During calm summer weather, tidal currents are presumed to dominate bottom shear stress over the flats. Assuming a sinusoidal particle excursion, maximum tidal current speed is given by $U_{T}=$ $\pi L / T$, where $T$ is the tidal period. A semi-diurnal period then gives $U_{T}=35 \mathrm{~cm} \mathrm{~s}^{-1}$, which is sufficient to mobilize unconsolidated sediment. Wells et al. (1990) measured maximum current speeds of $\sim 40 \mathrm{~cm} \mathrm{~s}^{-1}$ over Korean tidal flats, which is consistent with the above estimate.

During the winter monsoon, Korean flats are exposed to extensive periods of large ocean swell (Wells and Park 1992), and wave-generated shear stress is presumed to dominate. The amplitude of orbital velocity for a shallow water wave is given by linear theory to be

$$
U_{W}=\frac{H}{2 h}(g h)^{1 / 2},
$$

where $H$ is wave height, $h$ is still water depth and $g$ is the acceleration of gravity. Using the tidal range to scale $h$, a storm swell of $H=2 \mathrm{~m}$ gives $U_{W}=120 \mathrm{~cm} \mathrm{~s}^{-1}$. Since $\tau \sim U^{2}$, shear stress generated by storm swell will be an order of magnitude larger than that generated by $U_{T}$, and $U_{W}$ will effectively dominate the net field. Since maximum shear stress generated by waves $(\tau w)$ has the potential to be much greater than maximum shear stress generated by tides $\left(\tau_{T}\right)$, one might expect a seasonal transition from tide- to wavedominated hypsometry to be largely erosional, and a transition from wave- to tide- . dominated hypsometry to be largely depositional.

In the following sections, $U$ is used as a proxy for $\tau$ in deriving equilibrium flat morphologies. In Section 5.2 conservation of mass is used to determine the distribution of $U_{T}$, whereas in Section 5.3 conservation of energy is used to determine the distribution of $U_{W}$. Tidal flat profiles are then adjusted until a uniform distribution of $U$ signifies equilibrium. 
5.2. Tidal currents

If the shore-normal length scale of the tidal flat is much shorter than the tidal wave length, then allowing tidal elevation $(\eta)$ to pump up and down uniformly across the tidal flat is a reasonable assumption. Phase lags generated by momentum are important in the generation of velocity asymmetries over tidal flats (Friedrichs et al. 1992). However a kinematic approach is useful at first order when examining only the magnitude of tidal velocity. In the past, kinematic approaches have been used successfully in the study of velocity distributions along short channels in tidal marshes (Boon 1975; Petick 1980).

Thus the governing equation applied tidal currents in the absence of wind waves is simply conservation of mass:

$$
\frac{\mathrm{d} \eta(t)}{\mathrm{d} t}+\frac{\partial}{\partial x}\left\{h(x, t) u_{T}(x, t)\right\}=0
$$

where $h$ is local depth, and $u_{T}$ is tidal velocity. Equation (5.2.1) also assumes tidal flow to be entirely one-dimensional, thus neglecting the role played by intertidal channels in concentrating the flow of water across the flats. Nonetheless, flow over tidal flats is often sheet-like, especially during the flood, even in the presence of intertidal channels (Wells and Park 1992).

The direction of peak flow over intertidal flats may be at an angle to the bathymetric contours (e.g., Evans and Collins 1975; Wright et al. 1982). Equation (5.2.1) does not require tidal currents to be perpendicular to the contours, but only requires currents to flow at a constant angle to the bathymetry. In such a situation the $x$-coordinate in $(5.2 .1)$ is simply oriented at the same angle as the velocity. In their study of tidal flats along the Wash in the U.K., Evans and Collins (1975) found a marked clockwise rotation of the tidal current throughout the tidal cycle. Although the analytic model presented here allows the tide to flow at an angle to the bathymetry, rotation of the current is not incorporated.

Integrating (5.2.1) to solve for $u_{T}$ gives

$$
u_{T}(x, t)=\frac{x_{f}(t)-x}{h(x, t)} \frac{\mathrm{d} \eta(t)}{\mathrm{d} t}
$$


where $x_{f}(t)$ is the boundary between the wetted and exposed portions of the flat, hereafter termed the tidal front (Figure 5.2). Channel depth is defined in terms of its time and spacedependent components:

$$
h(x, t)=\eta(t)-Z(x)
$$

where $Z(x)$ is the local elevation of the tidal flat profile. Equations (5.2.2) - (5.2.3) hold for any flat lacking along-shore variations. If $\eta(t)$ and $Z(x)$ are specified, then $h(x, t), x_{f}(t)$ may be calculated, and a solution for $u_{T}$ may be found from (5.2.2).

The parameter $U_{T}(x)$ is defined as the maximum value reached by $u_{T}$ at each point in $x$ during the tidal cycle. According to the morphodynamic model applied in this chapter (see Section 5.1.2), if $U_{T}$ is uniform in $x$, then $\tau_{T}$ is uniform also. In the absence of wind waves, the tidal flats are at morphologic and hypsometric equilibrium. If $U_{T}$ (and therefore $\tau_{\Gamma}$ ) varies in $x$, then erosion is more likely (or deposition is less likely) in areas with larger $U_{T}$-- and vice versa for areas with smaller $U_{T}$. Under ideal conditions, erosion and/or deposition will continue until $U_{T}$ and $\tau_{T}$ become uniform.

The following sub-sections first consider tidal currents on a linearly sloping tidal flat along a straight shoreline. Next the tidal flat profile is adjusted to produce a uniform spatial distribution of $U_{T}$. Finally the role of shoreline curvature is considered. The uniqueness of the resulting equilibrium profiles will not be proven. The goal of this section is merely a description of simple profile forms over which $U_{T}$ and $\tau_{T}$ are constant in space.

In each case it is assumed that

$$
\eta(t)=a \sin \omega t,
$$

where $a$ is tidal amplitude, and $\omega$ is tidal frequency. However a similar approach may be used for more complex time-variations in elevation, including the effects of tidal harmonics in $\eta(t)$. The kinematic role of tidal harmonics in determining flow patterns has been considered along short marsh channels by Boon (1975) and Pethick (1980) and in the inner portion of longer tidal channels by Fry and Aubrey (1990). The kinematic ro:e of tidal harmonics in shaping equilibrium flat hypsometry is the subject of continuing work.

\subsubsection{Straight shoreline, linear profile}

Figure 5.2 displays a linearly sloping tidal flat with a shore-normal length of $L$ from the low to high water line. The tidal flat profile is given by 


$$
Z(x)=a(2 x / L-1)
$$

where $x=0$ at the low water line, and $Z=0$ at $x=L / 2$ (Figure 5.2). For a linear flat, evaluation of (5.2.2) is particularly straightforward. If the gradient of the flat is constant, then

$$
\frac{x_{f}(t)-x}{h(x, t)}=\text { constant }=\frac{L}{2 a}
$$

and

$$
u_{T}(t)=\frac{L}{2 a} \frac{\mathrm{d} \eta}{\mathrm{d} t} \cos \omega t=\frac{L \omega}{2}\left(1-\frac{\eta^{2}}{a^{2}}\right)^{1 / 2} .
$$

From (5.2.7) it is clear that maximum tidal velocity will occur when $\eta^{2}$ is at a minimum. For $x \leq L / 2, \eta^{2}$ is at a minimum when $\eta=0$. Thus for $x \leq L / 2$,

$$
U_{T}=L \omega / 2
$$

and maximum tidal velocity occurs at mid-tide. For $x>L / 2$, however, the smallest value of $\eta$ which maintains water at $x$ is (asymptotically) $\eta=Z$. Thus for $x>L / 2$,

$$
U_{T}(x)=\frac{L \omega}{2}\left(1-\frac{Z(x)^{2}}{a^{2}}\right)^{1 / 2}=L \omega\left(\frac{x}{L}-\frac{x^{2}}{L^{2}}\right)^{1 / 2},
$$

and maximum tidal velocity occurs at the tidal front. Thus (5.2.9) may be alternately expressed for $x>L / 2$ as

$$
U_{T}(x)=\frac{\mathrm{d} x_{f}}{\mathrm{~d} t} \text { when } \quad x=x_{f}
$$

Note that $(5.2 .8)-(5.2 .10)$ are all independent of tidal amplitude.

Figure 5.3 shows $U_{T}(x) / U_{T 0}$ and $\tau_{T}(x) / \tau_{T}$ as a function of $x / L$ across a linearly sloping flat. For $x / L \leq 1 / 2, U_{T}$ and $\tau_{T}$ are constant, suggesting that (in the absence of wind waves) a linear profile is at morphologic equilibrium over the seaward half of the flat. If values are chosen appropriate to the southwest coast of Korea $\left(M_{2}\right.$ tide, $\left.L=5 \mathrm{~km}\right)$ then $U T_{0}=35 \mathrm{~cm} / \mathrm{sec}$, which is large enough to mobilize fine sediment. If the water flows at an angle to the shore, $U_{T_{0}}$ is potentially higher. For $x / L>1 / 2$, however, there is a dramatic decrease in $U_{T}$ and $\tau_{T}$ as $x / L$ approaches 1 . Thus according to the morphodynamic model applied in this study, a linearly sloping flat with a stress field dominated by tidal currents alone is not at equilibrium for $x / L>1 / 2$. Greater deposition (or less erosion) should occur on the landward half of the flat until $U_{T}$ and $\tau_{T}$ become nearly uniform across the entire flat. 
5.2.2. Straight shoreline, equilibrium profile

A tidal flat profile is now derived which results in a uniform distribution of $U_{T}$ across the entire flat. Figure 5.4 displays a profile which is linear for $x \leq L^{*}$ and nonlinear for $x>L^{*}$. $L^{*}$ is defined such that $Z=0$ at $x=L^{*}$. The elevation of the lower tidal flat profile is given by

$$
Z_{.}(x)=a\left(x / L^{*}-1\right) \text { for } x \leq L^{*} \text {, }
$$

where $L^{*}$ is also the length of the lower profile. From the previous section it follows that for $x \leq L^{*}, u_{T}=L^{*} \omega$ at mid-tide and, therefore, $U_{T}$ is at least as large as $L^{*} \omega$. From the results of the previous section, it also seems reasonable to assume that for $x>L^{*}, U_{T}$ occurs at the tidal front. The next step is therefore to determine what $Z$ is required to give

for $x>L^{*}$.

$$
\frac{\mathrm{d} x_{f}}{\mathrm{~d} t}=L^{* \omega}
$$

Following a particle at the tidal front:

$$
\frac{\mathrm{d} x_{f}}{\mathrm{~d} \eta}=\frac{\mathrm{d} x_{f}}{\mathrm{~d} t} \frac{\mathrm{d} t}{\mathrm{~d} \eta}
$$

From (5.2.4),

$$
\frac{\mathrm{d} t}{\mathrm{~d} \eta}=\frac{1}{\omega} \frac{\mathrm{d}}{\mathrm{d} \eta}\left\{\arcsin \frac{\eta}{a}\right\}
$$

Utilizing (5.2.12) and (5.2.14) and integrating (5.2.13) then gives

$$
x_{f}-L^{*}=L^{*} \arcsin \eta / a .
$$

At the tidal front, $x=x_{f}, h=0$, and, from (5.2.3), $Z=\eta$. Eliminating $\eta$ and $x_{f}$ in (5.2.15) and solving for $Z$ then gives

$$
Z_{+}(x)=a \sin \left(x / L^{*}-1\right) \text { for } x>L^{*} .
$$

Since $Z_{+}=a$ at $x=L$, from (5.2.16) it is clear that for an equilibrium flat along a straight shoreline,

$$
L^{*} / L=(\pi / 2+1)^{-1}
$$

Figure 5.5 compares the equilibrium profile given by (5.2.11) and (5.2.16) with the linear profile given by (5.2.5) and indicates that the upper equilibrium profile is convex 
relative to the linear profile. For a straight shoreline, tidal flat hypsometry is equivalent to the tidal flat profile. Thus the results of this section indicate that in the absence of wind waves, tidal currents favor a convex hypsometry at equilibrium, which is consistent with the general observational trends presented in Section 5.1.

Finally, (5.2.17) can be used to constrain the equilibrium length of a tidal flat in the absence of wind waves if there exists some characteristic magnitude of $U_{T}$ at equilibrium. If $U_{T}=u_{e q}$, where $u_{e q}$ is some (externally fixed) velocity at equilibrium, then (5.2.17), along with the relation $U_{T}=L^{*} \omega$, yields

$$
L=(\pi / 2+1) u_{e q} / \omega
$$

Since (5.2.18) is independent of tidal amplitude, why do macrotidal flats generally cover larger areas than do mesotidal flats? Vegetation, which has so far been neglected in this paper, undoubtedly plays some role. Salt marshes are sensitive to tide range and generally do not extend below about one meter of the mean high water line (Frey and Basan 1985). Because of their sensitivity to tidal range, salt marshes clearly will reduce the equilibrium area of intertidal flats more substantially in micro- to mesotidal environments (range $<4 \mathrm{~m}$ ) than in macrotidal areas (range $>4 \mathrm{~m}$ ). For example, in Great Bay, New Hampshire (mean range $2 \mathrm{~m}$ ) extensive intertidal flats are limited to areas below mean water (Friedrichs et al. in prep., a). Of course wind-waves also play a role in determining the equilibrium width of intertidal flats. Their role will be discussed in more detail in Section 5.3 .

The above "vegetation effect" may partially explain the increase in convexity associated with increased tidal range by Dieckmann et al. (1987). The convex portion of the tidal profile in Figure 5.5 is limited to that area above mean water. If a large percentage of the intertidal area above mean water is vegetated, as might be expected along a mesotidal shoreline, then the convexity of the hypsometry will necessarily be reduced. Along a macro-tidal shoreline, however, vegetation will be viable over less of the convex portion of the profile.

Enuation (5.2.18) seems to suggest that equilibrium tidal flats that are dominated by $\tau_{T}$ are large. If $u_{e q}=30 \mathrm{~cm} \mathrm{~s}^{-1}$ during an $\mathrm{M}_{2}$ tide, then (5.2.18) gives $L=5.5 \mathrm{~km}$. It should be remembered, however, that $L$ is the length of the flat in the direction of the maximum tidal velocity. Thus it is only necessary for the component of the flat perpendicular to the bathymetric contours to be of length $L \cos \theta$, where $\theta$ is the angle 
between the velocity and the shoreline. Also, intertidal flats may not extend all the way to high water, but rather abut salt marsh. If a flat along a straight shoreline extends from low water to mean water, for example, it need only have a length parallel to the velocity direction of $L^{*}=u_{e q} / \omega$. If $u_{e q}=30 \mathrm{~cm} \mathrm{~s}^{-1}$, the flat in question lies completely below mean water, and $U_{T}$ is at a $45^{\circ}$ angle with the shoreline, then the shore-normal component of the flat need only extend $1.5 \mathrm{~km}$.

\subsubsection{Curved shoreline, equilibrium flat}

The effect of shoreline curvature on the distribution of $u_{T}$ over tidal flats is now considered. Equilibrium tidal profiles are derived and then re-expressed in terms of equilibrium hypsometries. Figure 5.6 provides plan views of "lobate" and "embayed" shorelines with tidal flats extending from $x=0$ to $x=L$. A lobate shoreline has a width at $x=L$ which is less than its width at $x=0$, giving $b_{L} / b_{0}<1$. An embayed shoreline has $b_{L} / b_{0}>1$, and a straight shoreline has $b_{L} / b_{0}=1$.

For a curved shoreline which is radially symmetric, continuity is most easily evaluated in polar coordinates:

$$
\frac{\mathrm{d} \eta}{\mathrm{d} t}+\frac{1}{r} \frac{\mathrm{d}}{\mathrm{d} x}\left\{r h(r, t) u_{T}(r, t)\right\}=0
$$

In this section it is assumed that tidal flow is everywhere perpendicular to the bathymetry. Equation (5.2.19) integrates to

$$
u_{T}(r, t)=\frac{r_{f}^{2}-r^{2}}{2 r h(r, t)} \frac{\mathrm{d} \eta}{\mathrm{d} t}
$$

where $r_{f}$ is the position of the tidal front. Keeping in mind that $b$ is proportional to $r$ (see Figure 5.6), (5.2.20) may be re-expressed as

$$
u_{T}(r, t)=\frac{1}{2}\left(b\left(r_{f}\right) / b(r)+1\right) \frac{r_{f}-r}{h(r, t)} \frac{\mathrm{d} \eta}{\mathrm{d} t}
$$

Transforming back to the $x$-coordinate, $r=r_{0} \pm x, r_{f}=r_{0} \pm x_{f}$, and $u_{T}(r, t)=$ $\pm u_{T}(x, t)$, where $r_{0}=r(x=0)$, and the \pm results from the shoreline being embayed $(+$, or lobate (-). Then (5.2.21) bacomes

$$
u_{T}(x, t)=B\left(x_{f}, x\right) \frac{x_{f}-x}{h(x, t)} \frac{\mathrm{d} \eta}{\mathrm{d} t}
$$

where 


$$
B\left(x_{f}, x\right)=\frac{1}{2}\left(b\left(x_{f}\right) / b(x)+1\right),
$$

and

$$
b(x) / b_{0}=1+\left(b_{L} / b_{0}-1\right) x / L
$$

Equations (5.2.22) - (5.2.24) hold for any radially symmetric flat, regardless of the precise form of the profile.

If shoreline curvature is negligible (i.e., $b_{L} / b_{0}=1$ ), then $\mathrm{b}\left(x_{f}\right)=b(x), B=1$, and (5.2.21) becomes identical to (5.2.2). If the shoreline is lobate $\left(b_{L} / b_{0}<1\right)$, then $b(x) \geq$ $b\left(x_{f}\right)>0$, and $B$ is bounded by $1 / 2<B \leq 1$. If the shoreline is embayed $\left(b_{L} / b_{0}>1\right)$, then $0<b(x) \leq b\left(x_{f}\right)$, and $B$ is bounded by $1 \leq B<\infty$. From the above bounds on $B$, it is clear that $B$ is less sensitive to lobate shorelines and more sensitive to embayed shorelines.

The derivation of equilibrium tidal flat profiles along curved shorelines in the absence of wind waves closely follows that used for straight shorelines. By analogy to Section 5.2.2, different relations are assumed to govern the equilibrium profile for $x \leq L^{*}$ and $x>L^{*}$. By further analogy with Section 5.2.2, it is assumed that $U_{T}=L^{*} \omega$ occurs simultaneously over all of $x \leq L^{*}$ when $x_{f}=L^{*}$. Unlike Section 5.2.2, however, $\eta$ is not assumed to be equal zero when $x_{f}=L^{*}$. Rather, $\eta=z^{*}$ when $x_{f}=L^{*}$, where $z^{*}$ may be less than or greater than zero, depending on the nature of the shoreline curvature. For $x>$ $L^{*}$, it is again assumed that $U_{T}$ occurs at the tidal front, i.e., $\mathrm{d} x_{f} / \mathrm{d} t=L^{*} \omega$.

In order to determine $z^{*},(5.2 .22)$ is evaluated at $x=0$ when $x_{f}=L^{*}$. Under these circumstances, (5.2.22) becomes

$$
U_{T}=L^{*} \omega=B\left(L^{*}, 0\right) \frac{L^{*}}{z^{*}+a} \frac{\mathrm{d} \eta\left(z^{*}\right)}{\mathrm{d} t} .
$$

Using the expression $\mathrm{d} \eta / \mathrm{d} t=a \omega\left(1-\eta^{2} / a^{2}\right)^{1 / 2}$, and solving for $z^{*} / a$ then gives:

$$
z^{*} / a=\frac{B\left(L^{*}, 0\right)^{2}-1}{B\left(L^{*}, 0\right)^{2}+1}
$$

If the shoreline is lobate, then $B<1$, and $z^{*}$ is negative. If the shoreline is embayed, then $B>1$, and $z^{*}$ is positive. Finally, if the shoreline is straight, $B=1$, and $z^{*}=0$.

The form of the tidal profile for $x \leq L^{*}$ is found by solving for $Z$. $(x)$ in (5.2.22) with $u_{T}=U_{T}, x_{f}=L^{*}$, and $\eta=z^{*}$. Then (5.2.22) becomes 


$$
L^{*} \omega=B\left(L^{*}, x\right) \frac{L^{*}-x}{z^{*}-Z_{-}(x)} a \omega\left(1-\frac{z^{* 2}}{a^{2}}\right)^{1 / 2}
$$

Or solving for Z./a,

$$
Z .(x) / a=z^{*} / a-B\left(L^{*}, x\right)\left(1-x / L^{*}\right)\left(1-\frac{z^{* 2}}{a^{2}}\right)^{1 / 2}
$$

for $x \leq L^{*}$. If the shoreline is straight, then $B=1, z^{*}=0$, and (5.2.28) becomes identical to $(5.2 .11)$.

For $x>L^{*}$, it is assumed that $U_{T}$ occurs at the tidal front, i.e., $\mathrm{d} x_{f} / \mathrm{d} t=U_{T}$. Preceding as in Section 5.2.2,

$$
\frac{\mathrm{d} x_{f}}{\mathrm{~d} \eta}=\frac{\mathrm{d} x_{f}}{\mathrm{~d} t} \frac{\mathrm{d} t}{\mathrm{~d} \eta}=L^{*} \omega \frac{1}{\omega} \frac{\mathrm{d}}{\mathrm{d} \eta}\left\{\arcsin \frac{\eta}{a}\right\}
$$

Integrating (5.2.29) gives

$$
x_{f}-L^{*}=L^{*}\left(\arcsin Z_{+} / a-\arcsin z^{*} / a\right)
$$

Solving for $Z_{+} / a$ in (5.2.30) then gives

$$
Z_{+}(x) / a=\sin \left\{\left(x / L^{*}-1\right)+\arcsin z^{*} / a\right\}
$$

for $x>L^{*}$. If $z^{*}=0,(5.2 .31)$ becomes equivalent to (5.2.16). Since $Z_{+}=a$ at $x=L$, (5.2.31) can be used to get an expression for $L^{*}$ relative to $L$ :

$$
L^{*} / L=\left(\pi / 2+1-\arcsin z^{*} / a\right)^{-1}
$$

If $z^{*}=0,(5.2 .32)$ reverts to (5.2.17).

Figure 5.7 displays equilibrium tidal profiles given by (5.2.28) and (5.2.31), plotted for both lobate $\left(b_{L} / b_{0}<1\right)$ and embayed $\left(b_{L} / b_{0}>1\right)$ shorelines, along with a linear profile for reference. The equilibrium nature of the profiles given by (5.2.28) and (5.2.31) was confirmed by using the profiles to solve (5.2.22) directly for $u_{T}(x, t)$. The maximum value of $u_{T}$ was found everywhere to be equal to $L^{*} \omega$. Figure 5.7 indicates that an embayed shoreline significantly enhances the convexity of the equilibrium tidal profile, whereas a lobate shoreline only slightly decreases the convexity of the profile. This behavior is consistent with the function $B$, given by (5.2.23), which is also more sensitive to embayed shorelines. 
Finally the profiles in Figure 5.7 are re-expressed as hypsometric curves, which are not equivalent to $Z(x)$ if the shoreline is curved. Hypsometric curves are plots of elevation versus cumulative basin area, $A$, where $A(x)$ is given by

$$
A(x)=\int_{x=0}^{x^{\prime}} b\left(x^{\prime}\right) \mathrm{d} x^{\prime} .
$$

Using (5.2.24) to eliminate $b,(5.2 .33)$ integrates to:

$$
\frac{A(x)}{A(L)}=\frac{2 x / L+\left(b_{L} / b_{0}-1\right)(x / L)^{2}}{1+b_{L} / b_{0}} .
$$

If the shoreline is straight, then $b_{L} / b_{0}=1$, and (5.2.34) reduces to $A(x) / A(L)=x / L$.

Figure 5.8 contains plots of $Z / a$ versus $A(x) / A(L)$ for the same values of $b_{L} / b_{0}$ plotted in Figure 5.7, along with a linear hypsometry for reference. Equilibrium hypsometries for embayed shorelines are much more convex than the corresponding profiles. Likewise, hypsometries for lobate shorelines are much less convex than the corresponding profiles -- so much so that the equilibrium hypsometry for a flat with $b_{L} / b_{0}$ $=1 / 4$ is primarily concave. The enhanced variation of hypsometries relative to profiles stems from the nonlinear hypsometric function given by (5.2.34).

To summarize the results of Section 5.2, it was first shown that the landward half of a linear profile dominated by tidal currents cannot be at equilibrium according to the morphodynamic model applied in this chapter. Next it was demonstrated that in the absence of wind waves, tidal currents favor a convex hypsometry at equilibrium, which is consistent with observations. If the shoreline is embayed, hypsometric convexity is increased, whereas if the shoreline is lobate, hypsometric convexity is decreased. The effect of shoreline curvature on equilibrium hypsometry is potentially as strong as the effect of domination by tidal currents.

Assuming a uniform distribution of $U_{T}$, it was also shown that tidal flat length and convexity at equilibrium should be independent of tidal range. It was speculated, however, that marsh vegetation may play a role in reducing flat length and convexity in micro- and mesotidal environments. Since the most convex portion of a flat dominated by tidal currents is the landward section, intertidal vegetation will disproportionately reduce convexity in small range environments. This may provide a partial explanation for the 
previously observed correlation between tidal range and convexity (Dieckmann et al. 1987).

\subsection{Wind waves}

The derivation for wave-dominated conditions parallels that described by Zimmerman (1973), who also examined the distribution of maximum bottom shear stress due to shoaling waves. The approach here differs in that Zimmerman did not apply the shallow water approximation nor did he consider waves shoaling across a linear profile.

The governing equation applied to wind waves in the absence of tidal currents is conservation of energy for monochromatic, remotely forced, forward propagating, shallow water surface waves:

$$
\frac{\mathrm{d}}{\mathrm{d} x}\left\{E(x) C_{g}(x)\right\}=-D(x)
$$

where $E$ is energy density, $C_{g}=(g h)^{1 / 2}$ is the wave group velocity, $g$ is the acceleration of gravity, and $D$ is dissipation by bottom friction. In this section wind waves are required to propagate perpendicular to the shoreline, thus avoiding the issue of refraction over the flat. It is assumed that sufficient refraction has occurred by the time waves reach the seaward edge of the flat that the remaining angle between the wave crest and the bathymetric contour is negligibly small.

In evaluating (5.3.1) neither the breaking waves nor wave energy reflected from the shoreline is considered. Thus this approach is inappropriate for highly energetic, steep beaches. However for wide, gently sloping, highly dissipative tidal flats, the approach should be adequate -- at least for gaining useful physical insight. It is also assumed that the largest waves are most likely to occur around the time of high water. This is a reasonable assumption in enclosed intertidal basins because fetch will be smaller near low tide. It is also a reasonable assumption for open coasts if subtidal topography continues to slope gently offshore. Then offshore dissipation will be much at lower tide levels, sharply reducing the height of waves impinging on the flats.

Energy density in (5.3.1) is given by

$$
E(x)=1 / 8 \rho g H(x)^{2}
$$

where $\rho$ is the fluid density, and $H$ is the wave height. Frictional dissipation in (5.3.1) is given by 


$$
D(x)=\frac{1}{T_{W}} \int_{-T_{W} / 2}^{T_{W} / 2}\left\{\rho c_{d} u_{W}\left|u_{W}\right|\right\} u_{W} \mathrm{~d} t
$$

where $T_{W}$ is the wave period, $u_{W}$ is instantaneous wave velocity, and the quantity in brackets is instantaneous wave-generated bottom shear stress. If the reasonable assumption is made that

$$
u_{W}(x, t)=U_{W}(x) \sin \left(2 \pi t / T_{W}\right)
$$

then substitution of (5.3.4) into (5.3.3) gives

$$
D(x)=\frac{\rho c_{d} U_{W}^{3}}{T_{W}} \int_{-T_{W} / 2}^{T_{W} / 2} \sin ^{2} 2 \pi t / T_{W}\left|\sin 2 \pi t / T_{W}\right| \mathrm{d} t
$$

which integrates to

$$
D(x)=\frac{4}{3 \pi} \rho c_{d} U_{W^{3}}
$$

The above relations for $E(x)$ and $D(x)$, along with the relation $C_{g}=(g h)^{1 / 2}$, are substituted into (5.3.1), yielding

$$
\frac{\mathrm{d}}{\mathrm{d} x}\left\{H(x)^{2} h(x)^{1 / 2}\right\}=-\frac{32}{3 \pi} g^{-3 / 2} c_{d} U_{W^{3}} .
$$

Equation (5.1.2) is then used to eliminate $H(x)$ from (5.3.7). The result is a first-order ordinary differential equation for for $U_{W}$ :

$$
\frac{1}{U_{W^{2}}} \frac{\mathrm{d}}{\mathrm{d} x} U_{W}+\frac{3}{4 h U_{W}} \frac{\mathrm{d} h}{\mathrm{~d} x}=-\frac{4 c_{d}}{3 \pi g^{1 / 2} h^{3 / 2}} .
$$

Equation (5.3.8) is solved more easily if it is rewritten as the following O.D.E for $U_{W^{-1}}$ :

$$
\frac{\mathrm{d}}{\mathrm{d} x} U_{W}^{-1}-\frac{3}{4 h} \frac{\mathrm{d} h}{\mathrm{~d} x} U_{W}^{-1}=\frac{4 c_{d}}{3 \pi g^{1 / 2} h^{3 / 2}} .
$$

The boundary condition on (5.3.9) is $U_{W^{-1}}=U_{W_{0}}{ }^{-1}$ at $x=0$, which may determined from $H(x=0)$ via (5.1.2). Assuming $h(x)$ is known, then (5.3.9) can be solved completely for $U_{W}$

An alternative approach in solving for $U_{W}$ during shoaling is to assume that increases in $H$ due to concentration of energy are balanced by decreases in $H$ due to dissipation. If this balance holds, then $H$ can be treated as a constant in (5.3.7), 
simplifying the solution for $U_{W}$. This approach was used by Wright et al. (1982) in their calculations of dissipation rates across a macrotidal beach. If $H$ is held constant, however, (5.2.1) indicates that $U_{W} \sim h^{-1 / 2}$ will always increase with decreased depth (seaward of the breaking point). Thus the morphologic model applied in this chapter suggests a wavedominated flat over which $H$ is constant cannot be at morphologic equilibrium. Allowing $H$ to vary in $x$ is therefore essential to realistically representing equilibrium profiles. The elimination of $H(x)$ in (5.3.7) via (5.1.2) allows dynamic variations in $H$ to be included implicitly in the solution for $U_{W}$.

In the following sub-sections (5.3.9) is first solved for wind waves impinging on a linearly sloping tidal flat. Next the tidal flat profile is derived which results in a uniform distribution of $U_{W}$. Finally the role of shoreline curvature is considered.

\subsubsection{Straight shoreline, linear profile}

Figure 5.9 displays a linearly sloping tidal flat of length $L$. As in Section $5.2, L$ is the shore-normal distance from the low to high water line. The depth of the tidal flat profile is given by

$$
h(x)=(L-x) h_{0} / L,
$$

where $h_{0}$ is the high-water depth at $x=0$ and also is equal to the tidal range. Substituting (5.3.10) into (5.3.9) yields

$$
(L-x) \frac{\mathrm{d}}{\mathrm{d} x} U_{W}^{-1}+\frac{3}{4} U_{W^{-1}}=C_{1}(L-x)^{-1 / 2} .
$$

where

$$
C_{1}=\frac{4 c_{d} L^{3 / 2}}{3 \pi g^{1 / 2} h_{0}^{3 / 2}}
$$

From the right hand side of (5.3.11), the particular solution for $U_{W^{-1}}$ is expected to have the form

$$
\left\{U_{W^{-1}}\right\}_{\text {part }}=C_{2}(L-x)^{-1 / 2}
$$

Substituting (5.3.13) into (5.3.11) yields $C_{2}=4 / 5 C_{1}$. The homogeneous portion of (5.3.1 i) may be re-expressed as:

$$
\frac{1}{U_{W}-1} \mathrm{~d} U_{W^{-1}}=-\frac{3}{4} \frac{1}{(L-x)} \mathrm{d} x
$$

which integrates to: 


$$
\left\{U_{W^{-1}}\right\}_{\text {homo }}=C_{2}(L-x)^{3 / 4} .
$$

Finally, the boundary condition at $x=0$ gives

$$
C_{3}=U_{W_{0}}{ }^{-1} L^{-3 / 4}-C_{2} L^{-5 / 4} \text {. }
$$

Combining the above equations yields the following solution for maximum orbital velocity due to wind waves impinging on a linearly sloping tidal flat:

$$
U_{W}(x) / U_{W_{0}}=\left\{\widehat{H}_{0}(1-x / L)^{-1 / 2}+\left(1-\widehat{H}_{0}\right)(1-x / L)^{3 / 4}\right\}^{-1},
$$

where the non-dimensional forcing wave height, $\widehat{H}_{0}$, is given by

$$
\widehat{H}_{0}=\frac{16}{15 \pi} c_{d} \frac{L}{h_{0}} \frac{U_{W_{0}}}{\left(g h_{0}\right)^{1 / 2}}=\frac{8}{15 \pi} c_{d} \frac{L H_{0}}{h_{0}^{2}} .
$$

Figure 5.10 shows $U_{W}(x) / U_{W_{0}}$ and $\tau w(x) / \tau w_{0}$ as a function of $x / L$ for various values of $\widehat{H}_{0}$ ranging from $\widehat{H}_{0}=0.1$ to $\widehat{H}_{0}=2$. No value of $\widehat{H}_{0}$ for a linearly sloping tidal flat results in a uniform distribution of $U_{W}$ across the flat. Thus, according to the morphodynamic model applied here, a linearly sloping flat dominated by wind waves cannot be at equilibrium.

Applying reasonable values for the southwest coast of Korea $\left(c_{d}=0.01, L=5 \mathrm{~km}\right.$, $h_{0}=6 \mathrm{~m}$ ), then calm weather waves with a height at $x=0$ of $H_{0}=50 \mathrm{~cm}$ give $U_{W_{0}} \approx 30$ $\mathrm{cm} / \mathrm{s}$ and $\widehat{H}_{0} \approx 0.1$. Figure 5.10 indicates that for $\widehat{H}_{0}=0.1, U_{W}$ and $\tau_{W}$ will increase with distance from $x / L=0$ to $x / L=0.9$. According to our morphodynamic model, more deposition (or less erosion) should occur over the lower profile than over the upper profile. Assuming a reasonable storm value of $H_{0}=2 \mathrm{~m}$, then $U_{W_{0}} \approx 1.3 \mathrm{~m} / \mathrm{s}$, and $\widehat{H}_{0} \approx 0.5$. For $\widehat{H}_{0}=0.5$, Figure 5.10 indicates $U_{W}$ and $\tau W$ will reach a maximum around $x / L=0.3$ and decrease from $x / L=0.3$ to $x / L=1$. Under storm conditions, greater erosion (or less deposition) should occur over the lower profile than over the upper profile. Thus (5.3.17) - (5.3.18) and Figure 5.10 may partially explain the seasonal oscillation in Korean tidal flat profile observed by Wells and Park (1991) to be a direct function of wind wave climate.

\subsubsection{Straight shoreline, equilibrium profile}

A tidal flat profile is now derived which results in a uniform distribution of $U_{W}$ across the entire flat (Figure 5.11). A similar solution for was found previously by Bruun 
(1954) who assumed energy dissipation to be uniform across an equilibrium shoreface. However Bruun did not consider the effect of shoreline curvature which is addressed in this study in Section 5.3.3.

Deriving an equilibrium profile with $U_{W}=U_{W_{0}}$ everywhere is actually much simpler than solving for $U_{W}(x)$. If $U_{W}$ is constant, the first term in (5.3.9) is zero, and (5.3.9) may be rewritten as

$$
h^{1 / 2} d h=-\frac{16}{9 \pi} c_{d} U_{W_{0}} g^{-1 / 2}(L-x)
$$

Equation (5.3.19) integrates to

$$
h(x) / h_{0}=(1-x / L)^{2 / 3},
$$

where $h_{0}$ is given by

$$
h_{0}=\left(\frac{8}{3 \pi} c_{d} U_{W_{0}} g^{-1 / 2} L\right)^{2 / 3} \text {. }
$$

The $2 / 3$ exponent in (5.3.20) has also been observed empirically for shoreface profiles of dissipative beaches along the U.S. Atlantic and Gulf Coasts (Dean 1977). Equation (5.3.21) can be crudely checked by comparison to the Korean values. If (5.3.21) is solved for $U_{W_{0}}$, then $c_{d}=0.01, L=5 \mathrm{~km}$, and $h_{0}=6 \mathrm{~m}$ give $U_{W_{0}}=1.1 \mathrm{~m} / \mathrm{s}$, which is a value that is certainly capable of mobilizing sediment. Using (5.1.2), this velocity is equivalent to a forcing wave height of $H_{0}=1.7 \mathrm{~m}$, which seems like a reasonable value for typical wave dominated conditions.

Figure 5.12 compares the equilibrium profile given by (5.3.20) with the linear profile given by (5.3.10) and indicates that the wave-dominated equilibrium profile is concave relative to the linear profile. For a straight shoreline, tidal flat hypsometry is equivalent to the tidal flat profile. Thus (5.3.20) indicates wind-waves favor a concave hypsometry at equilibrium, which is consistent with the observations summarized in Section 5.1 .

Finally, (5.3.21) can be used to derive the equilibrium length for a flat under wavedominated conditions. If $H_{0}, h_{0}$ and $c_{d}$ are considered to be characteristic values, independent of the extent of the tidal flat, then (5.3.21) and (5.1.2) can be combined and solved for $L$ :

$$
L=\frac{3 \pi}{4 c_{d}} \frac{h_{0}^{2}}{H_{0}}
$$


Equation (5.3.22) seems qualitatively sensible in that it indicates that equilibrium tidal flat width decreases with greater wave height, $H_{0}$, and increases dramatically with greater tidal range, $h_{0}$. Equation (5.3.22) suggests that the position of the low tide line should oscillate with seasonal variations in forcing wave height. This predicted oscillation is qualitatively consistent with the observations of Wells and Park (1992).

Equation (5.3.22) may also help explain the associations of small tidal ranges with concave hypsometry and of large tidal ranges with convex hypsometry (Dieckmann et al. 1987). If wave height is moderate and tidal range is small, (5.3.22) indicates that $L$ will also be small. In Section 5.2, $U_{T}$ was found to be directly proportional to $L$. Thus if $L$ is small, $U_{T}$ will be small also. Under these conditions, $U_{W}$ and $\tau_{W}$ will dominate $U_{T}$ and $\tau_{T}$, and the equilibrium profile will be concave. If tidal range is large and waves are moderate, then (5.3.22) indicates $L$ will be much larger (since $L$ is geometrically dependent on $h_{0}$ ). Since $U_{T}$ is proportional to $L, U_{T}$ will also be much larger. $U_{W}$ and $\tau_{W}$ may no longer dominate $U_{T}$ and $\tau_{T}$, at least under fair weather conditions, and the equilibrium profile may be expected to be more convex.

\subsubsection{Curved shoreline, equilibrium profile}

The effect of shoreline curvature on the distribution of $U_{W}$ over tidal flats is now examined. As in Section 5.2, equilibrium profiles are derived first and then re-expressed in terms of equilibrium hypsometries. Lobate and embayed shorelines are again considered as described by Figure 5.6.

For a curved shoreline which is radially symmetric, conservation of energy is most easily evaluated in polar coordinates:

$$
\frac{1}{r} \frac{\mathrm{d}}{\mathrm{d} r}\left\{r E(r) C_{g}(r)\right\}=-D(r)
$$

It is assumed that refraction has already caused the wind waves to propagate nearly perpendicular to the bathymetric contours by the time the waves reach the edge of the flat at $r_{0}=r(x=0)$. Thus refraction is not considered over the flat.

Evaluation of (5.3.23) is straightforward if $U_{W}=U_{W_{0}}$ equals a constant over the entire profile. Using $(5.1 .2),(5.3 .2),(5.3 .6)$ and the relation $C_{g}=(g h)^{1 / 2},(5.3 .23)$ becomes

$$
\frac{\mathrm{d}}{\mathrm{d} r}\left\{r h^{3 / 2}\right\}=-\frac{8}{3 \pi} c_{d} U_{W_{0}} g^{-1 / 2} r
$$


Equation (5.3.24) integrates to

$$
r h^{3 / 2}=\frac{4}{3 \pi} c_{d} U_{W_{0}} g^{-1 / 2}\left(r_{L}^{2}-r^{2}\right),
$$

where $r_{L}=r(x=L)$. Keeping in mind that $b$ is proportional to $r$ (see Figure 5.6), (5.3.25) may be re-expressed as

$$
h(r)^{3 / 2}=\frac{1}{2}\left(b\left(r_{L}\right) / b(r)+1\right) \frac{8 c_{d} U_{W_{0}}}{3 \pi g^{1 / 2}}\left(r_{L}-r\right),
$$

Transforming back to the $x$-coordinate, (5.3.26) becomes

$$
h(x) / h_{0}=\{B(L, x) / B(L, 0)\}^{2 / 3}(1-x / L)^{2 / 3},
$$

where

$$
h_{0}=\left(B(L, 0) \frac{8}{3 \pi} c_{d} U_{W_{0}} g^{-1 / 2} L\right)^{2 / 3} \text {, }
$$

and

$$
B(L, x)=\frac{1}{2}\left(b_{L} / b(x)+1\right) .
$$

$B(L, x)$ is analogous to $B\left(x_{f}, x\right)$ in (5.2.23) of Section 5.2, and $b(x)$ above is identical to (5.2.24). If shoreline curvature is negligible (i.e., $b_{L} / b_{0}=1$ ), then $b_{L}=b(x), B=1$, and $(5.3 .27)$ - (5.3.28) become identical to (5.3.20) - (5.3.21).

Figure 5.13 displays equilibrium wave-dominated profiles given by (5.3.27), plotted for both lobate $\left(b_{L} / b_{0}<1\right)$ and embayed $\left(b_{L} / b_{0}>1\right)$ shorelines, along with a linear profile for reference. Figure 5.13 indicates that a lobate shoreline only slightly increases the concavity of the profile, whereas an embayed shoreline greatly decreases the concavity of the profile -- so much so that the equilibrium profile for a flat with $b_{L} / b_{0}=1 / 4$ is primarily convex. The greater sensitivity of the profile to embayed shorelines is similar to that seen in Section 5.2 (see Figure 5.7). As discussed in Section 5.2, this follows from a dependence of the equilibrium profile on the function $B$, which is also more sensitive to embayed shorelines.

Finally, the profiles in Figure 5.13 are re-expressed as hypsometric curves. Figure 5.14 contains plots of $h / h_{0}$ vs. $A(x) / A(L)$ for the same values of $b_{L} / b_{0}$ plotted in Figure 5.13 , along with a linear hypsometry for reference. The formula for $A(x) / A(L)$ is given by (5.2.34). Equilibrium hypsometries for lobate shorelines are significantly more concave than the corresponding profiles. Likewise, hypsometries for embayed shorelines are 
significantly less concave than the corresponding profiles. In fact, the hypsometries for $b_{L} / b_{0} \leq 1 / 2$ are primarily convex. The enhanced variation of hypsometries relative to profiles stems from the nonlinear hypsometric function given by (5.2.34).

To summarize the results of Section 5.3, it was first shown that a wave dominated linear profile cannot be at equilibrium according to the morphodynamic model applied in this chapter. Next it was demonstrated that a bottom stress field dominated by wind waves favors a concave hypsometry at equilibrium, which is consistent with observations. If the shoreline is embayed, hypsometric concavity is decreased, whereas if the shoreline is lobate, hypsometric concavity is increased. The effect of shoreline curvature on equilibrium hypsometry is potentially as strong as the effect of domination by wind waves.

Assuming a uniform distribution of $U_{W}$, a compact expression was also found for the length of an equilibrium flat, suggesting $L \sim h_{0}^{2} / H_{0}$, where $h_{0}$ is the tidal range and $H_{0}$ is the forcing wave height. The above expression indicates that the width of equilibrium flats should increase dramatically with tidal range. Since $U_{T}$ is proportional to $L$ (see Section 5.2), the relative importance of tidal currents should also increase strongly with tidal range, providing a further explanation for the previously observed transition from concave to convex hypsometry with increasing tidal range (Dieckmann et al. 1987).

\subsection{Summary and conclusions}

Recent observations of tidal flat hypsometry have correlated convexity with large tide ranges, long-term accretion and/or low wave activity. Concavity, in turn, has been correlated with small tide ranges, long-term erosion and/or high wave activity. This study demonstrates that much of this empirically observed variation in tidal flat hypsometry may be explained by a simple morphodynamic model which assumes tidal flats to be at equilibrium if maximum shear stress is uniform in space. Assuming a constant drag coefficient, this condition is equivalent to a uniform distribution of maximum velocity.

In the absence of wind waves, maximum velocity is given by maximum tidal velocity, $U_{T}$. Assuming the tide to pump iniformly, continuity may be solved kinematically to determine $U_{T}$ as a function of distance across the flat. The simplest case is for flow perpendicular to the shoreline, but similar arguments hold for flow at a constant angle to the shore. For a flat which slopes linearly away from a straight shoreline, results show that $U_{T}$ is constant over the seaward half of the flat. Therefore the lower portion of a 
linearly sloping flat is potentially at morphologic equilibrium. Over the landward half, however, a dramatic decrease in $U_{T}$ is predicted, indicating disequilibrium.

Along a straight, waveless shoreline, the equilibrium profile has a linear lower portion which produces uniform $U_{T}$ over the seaward portion of the flat. Over the landward portion of the flat, $U_{T}$ is assumed to occur at the tidal front, $x_{f}$. The equilibrium upper profile is chosen so that $\mathrm{d} x_{f} / \mathrm{d} t$ is constant and equal to the $U_{T}$ determined for the lower profile. The resulting profile is convex overall and demonstrates that tidal currents favor convex hypsometry. The equilibrium value for $U_{T}$ is proportional to the length of the tidal flat but independent of tidal range. Since the equilibrium profile is most strongly convex above the mean water line, marsh vegetation extending a finite distance below the high water line may tend to reduce observed hypsometric convexity, especially for flats subject to relatively small tidal ranges.

In the absence of waves, an equilibrium flat along a curved shoreline is derived in a similar manner. Results indicate that an embayed shoreline significantly enhances the convexity of the equilibrium profile, whereas a lobate shoreline only slightly decreases the convexity. The nonlinear transformation from profiles to hypsometries, however, causes the hypsometry of embayed and lobate shorelines to be much more or much less convex than the corresponding profiles -- so much so that the effect of shoreline curvature on equilibrium hypsometry is potentially as strong as the effect of domination by tidal currents.

In the presence of wind waves, maximum velocity is often dominated by the maximum wave orbital velocity, $U_{W}$. Assuming dissipative shallow water waves impinging at high water, conservation of energy is utilized to determine $U_{W}$ as a function of distance across the entire flat. The resulting expression is a first-order ordinary differential equation for $U_{W^{-1}}$. For a flat sloping linearly away from a straight shoreline, the solution may be expressed in terms of a single dimensionless forcing wave height, $\widehat{H}_{0}$. No value of $\widehat{H}_{0}$ results in a uniform distribution of $U_{W}$, thus no part of a linearly sloping, wavedominated flat is at equilibrium.

An equilibrium flat along a straight, wave-dominated shoreline is derived by setting $U_{W}$ constant in the previously derived governing equation for $U_{W}{ }^{-1}$. The resulting equilibrium profile has depth increasing like $x^{2 / 3}$, a form which has been reported empirically for dissipative beaches and which demonstrates that wind waves favor concave hypsometry. The equilibrium profile length, $L$, is proportional to $h_{0} 2 / H_{0}$, where $h_{0}$ is the 
tidal range and $H_{0}$ is the forcing wave height. This expression indicates that $L$ should increase dramatically with tidal range. Since $U_{T} \sim L$, the relative importance of tidal currents should also increase strongly with tidal range, favoring a transition form concave to convex hypsometry with increasing tidal range.

An equilibrium flat along a curved, wave-dominated shoreline is derived in a similar manner. Similar to the no wave case, results indicate that an embayed shoreline significantly decreases the concavity of the profile -- potentially to the point of convexity -whereas a lobate shoreline only slightly increases concavity. Again, the nonlinear transformation from profiles to hypsometries causes the hypsometries to be much more or much less concave than the profiles. 
Figure captions for Chapter 5

Figure 5.1. Block diagrams of idealized topographies along with associated hypsometric curves. Modified from Boon and Byme (1981).

Figure 5.2. Schematic side view of a linearly sloping flat along a straight shoreline which is dominated by tidal currents. $\eta$ is tidal elevation, $h$ is local depth, $x_{f}$ is the position of the tidal front, $Z$ is the elevation of the profile.

Figure 5.3. Maximum (a) tidal velocity and (b) tidally-generated shear stress as a function of distance across a flat which slopes linearly away from a straight shoreline.

Figure 5.4. Schematic side view of an equilibrium flat along a straight shoreline which is dominated by tidal currents.

Figure 5.5. Profile of an equilibrium flat along a straight shoreline dominated by tidal currents. The equilibrium profile is convex relative to the linear profile. Hypsometry is equivalent to the profile along a straight shoreline.

Figure 5.6. Schematic plan view of a lobate and an embayed shoreline. The contours $0-4$ are arbitrary heights between low and high water.

Figure 5.7. Profiles of equilibrium flats along curved shorelines dominated by tidal currents. The equilibrium profiles are all convex relative to the linear profile.

Figure 5.8. Hypsometries of equilibrium flats along curved shorelines dominated by tidal currents. $b_{L} / b_{0}=1 / 2-4$ are convex, $b_{L} / b_{0}=1 / 4$ is concave.

Figure 5.9. Schematic side view of a linearly sloping flat along a straight shoreline which is dominated by tidal currents. $H_{0}$ is offshore wave height, $C_{g}$ is wave velocity, $h$ is local depth at high water, $h_{0}$ is high water depth at $x=0$ and also the tidal range.

Figure 5.10 Maximum (a) wave orbital velocity and (b) wave-generated shear stress as a function of non-dimensional offshore wave height and of distance across a flat which slopes linearly away from a straight shoreline.

Figure 5.11. Schematic side view of an equilibrium flat along a straight shoreline which is dominated by wind waves. 
Figure 5.12. Profile of an equilibrium flat along a straight shoreline dominated by wind waves. The equilibrium profile is concave relative to the linear profile. Hypsometry is equivalent to the profile along a straight shoreline.

Figure 5.13. Profiles of equilibrium flats along curved shorelines dominated by wind waves. $b_{L} / b_{0}=1 / 4-2$ are concave, $b_{L} b_{0}=4$ is convex.

Figure 5.14. Hypsometries of equilibrium flats along curved shorelines dominated by wind waves. $b_{L} / b_{0}=1 / 4-1$ are concave, $b_{L} / b_{0}=2-4$ are convex. 
Topography

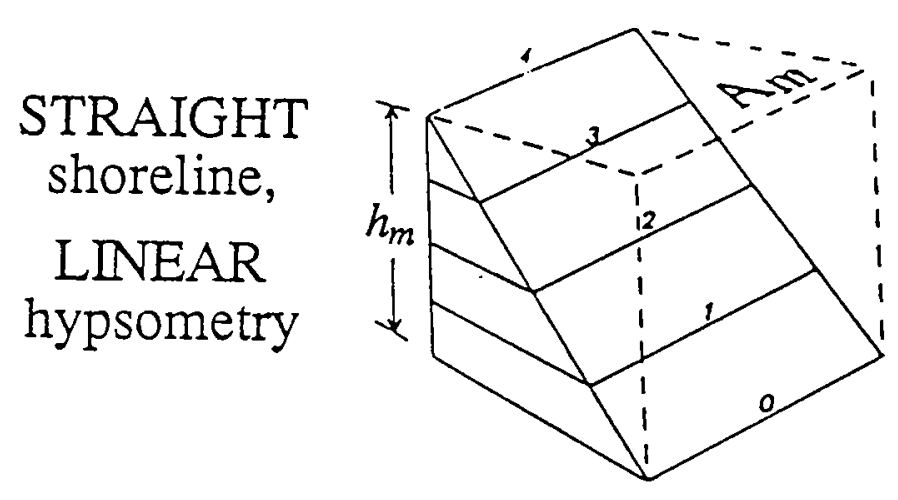

EMBAYED shoreline, CONVEX hypsometry

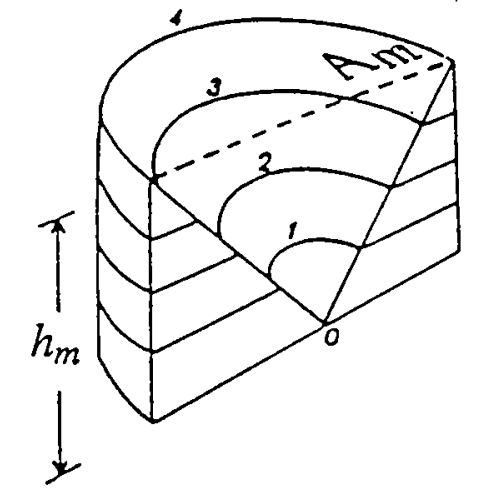

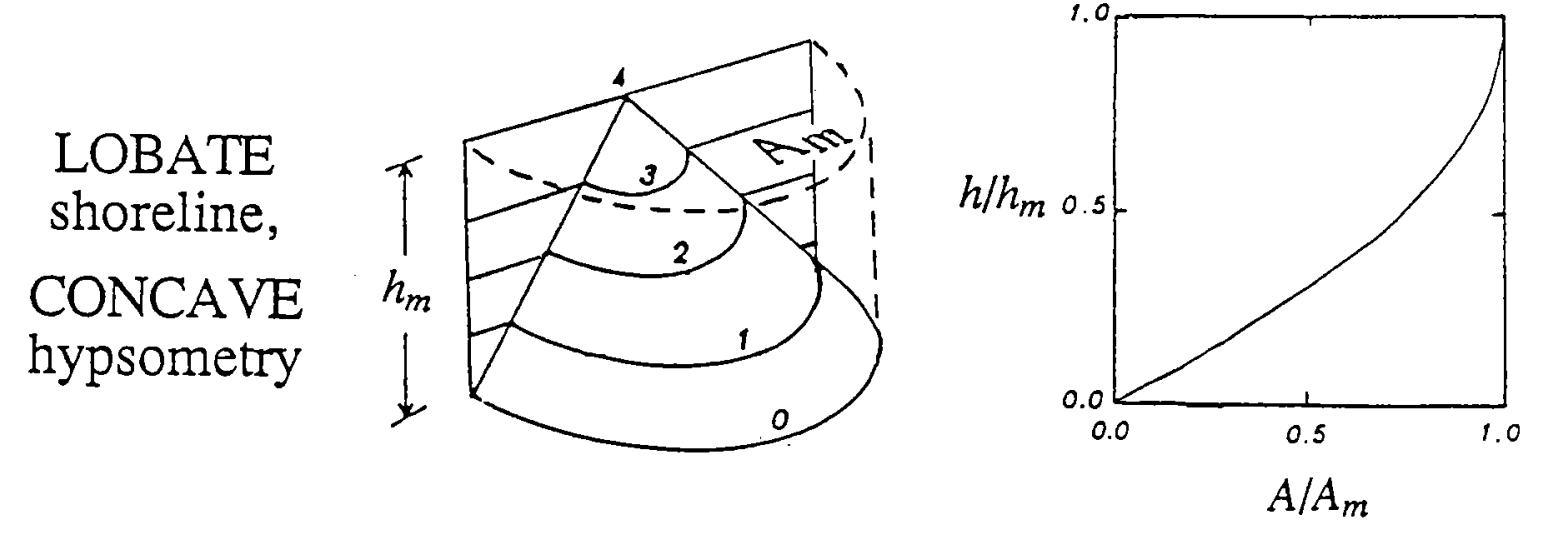

Hypsometry
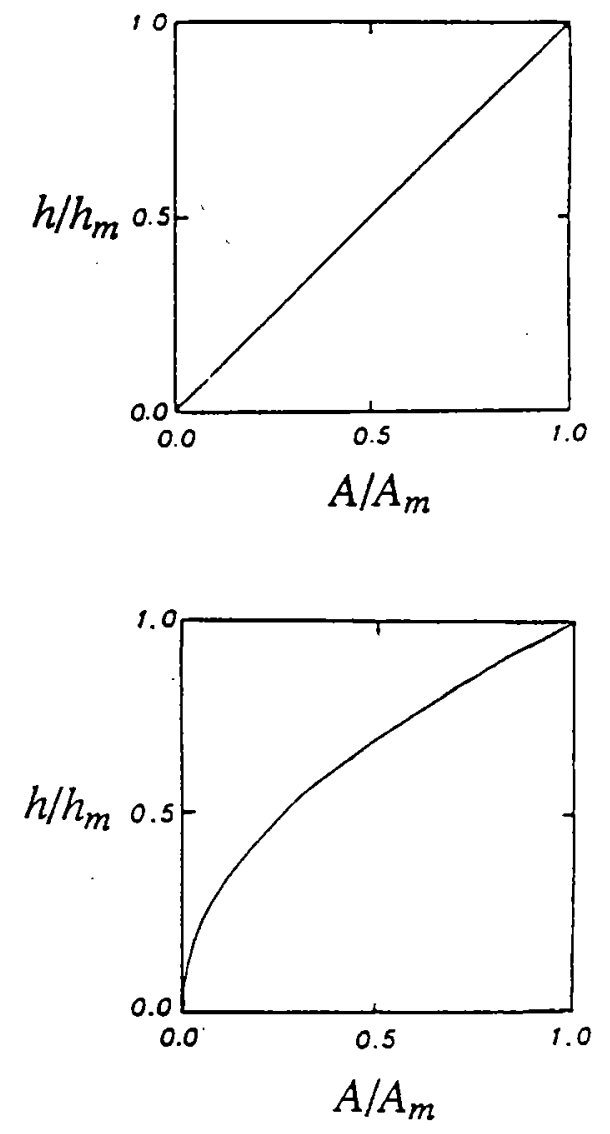

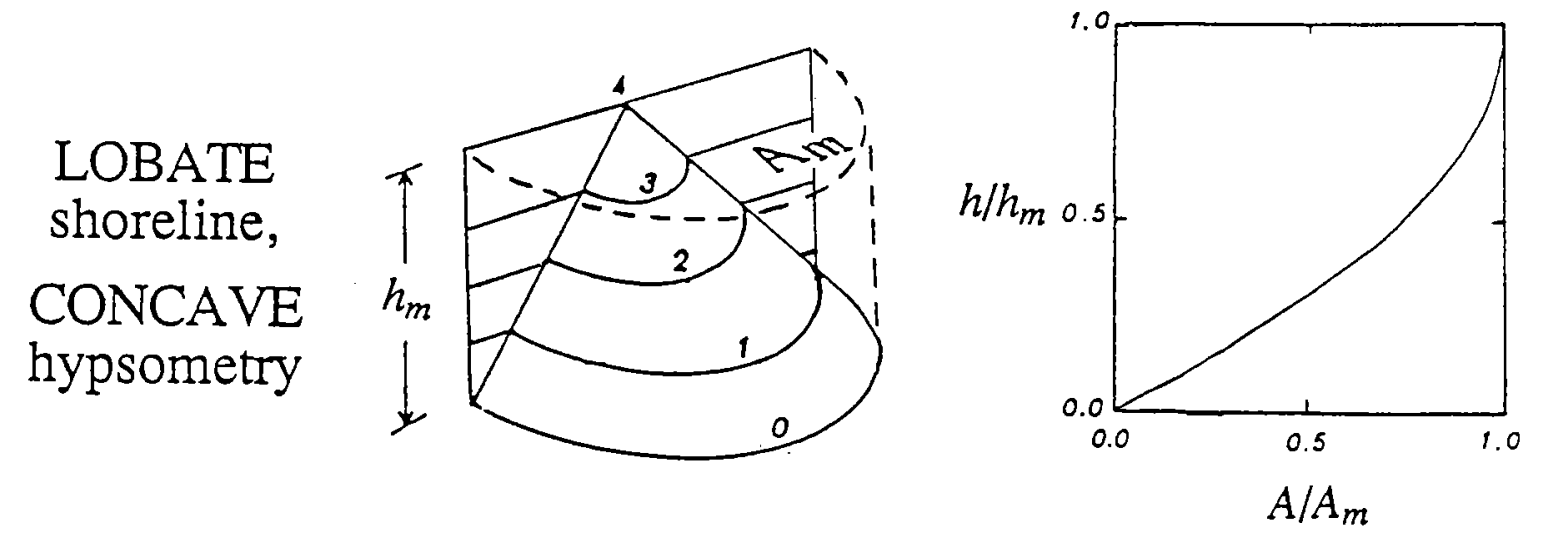

Figure 5.1

LOBATE shoreline, CONCAVE hypsometry 
Tidal current dominated linear flat profile: Definitions

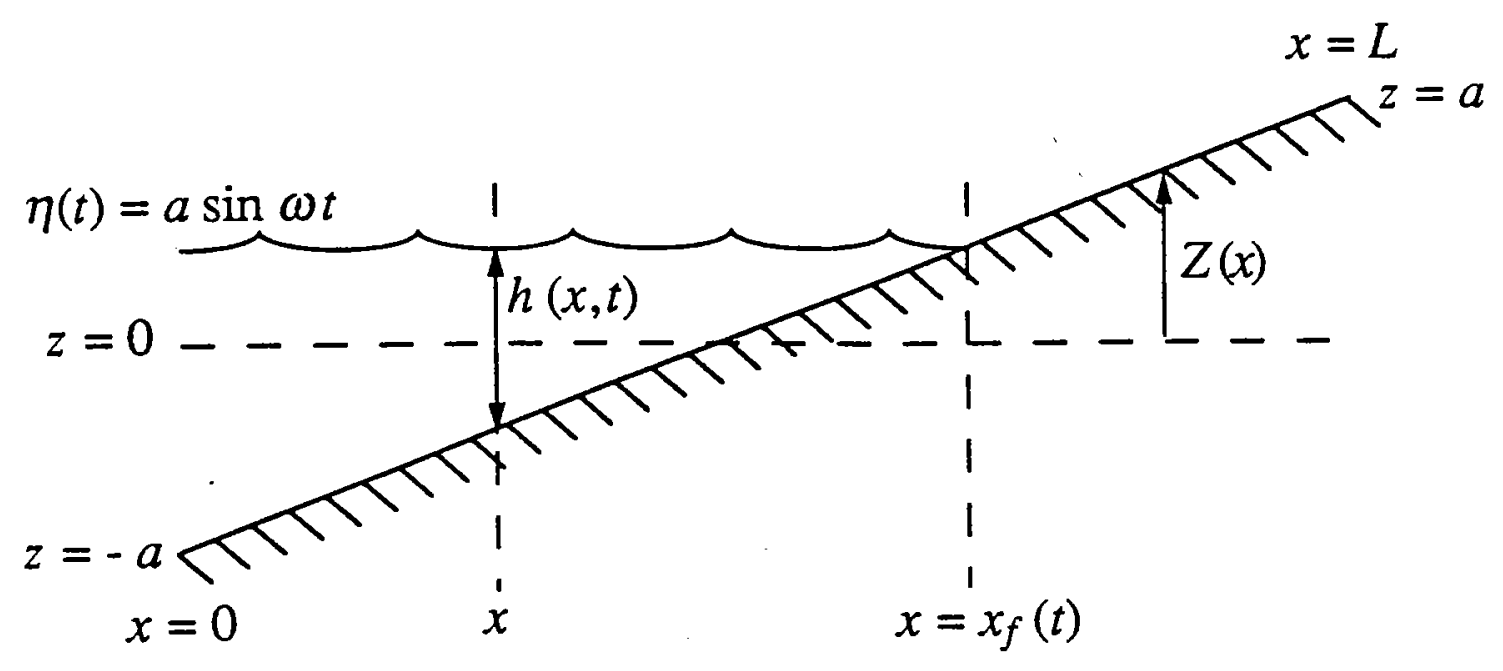

Figure 5.2 
$U_{T}$ and $\tau_{T}$ across a linearly sloping flat:
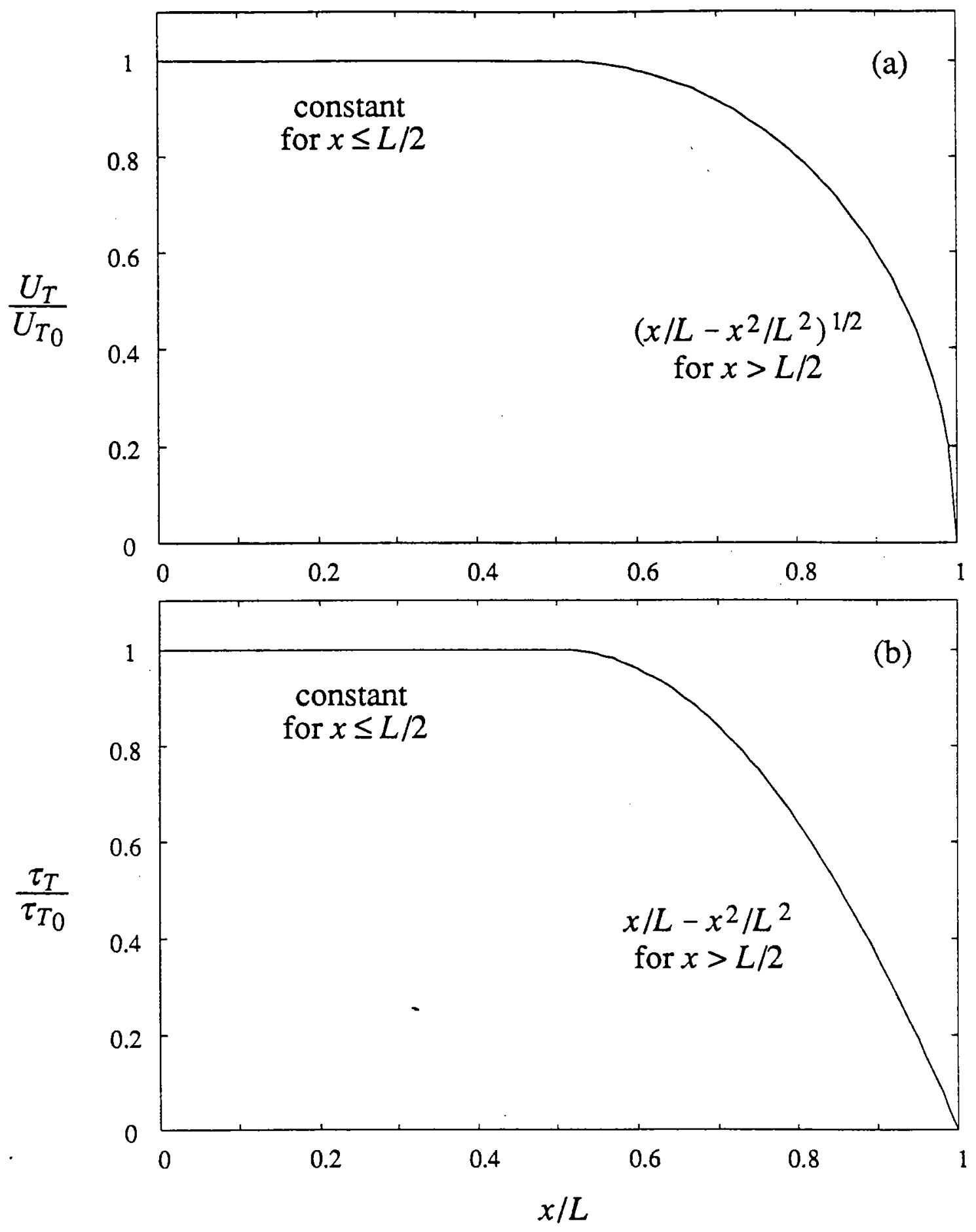

Figure 5.3 
Tidal current dominated non-linear flat profile: Definitions

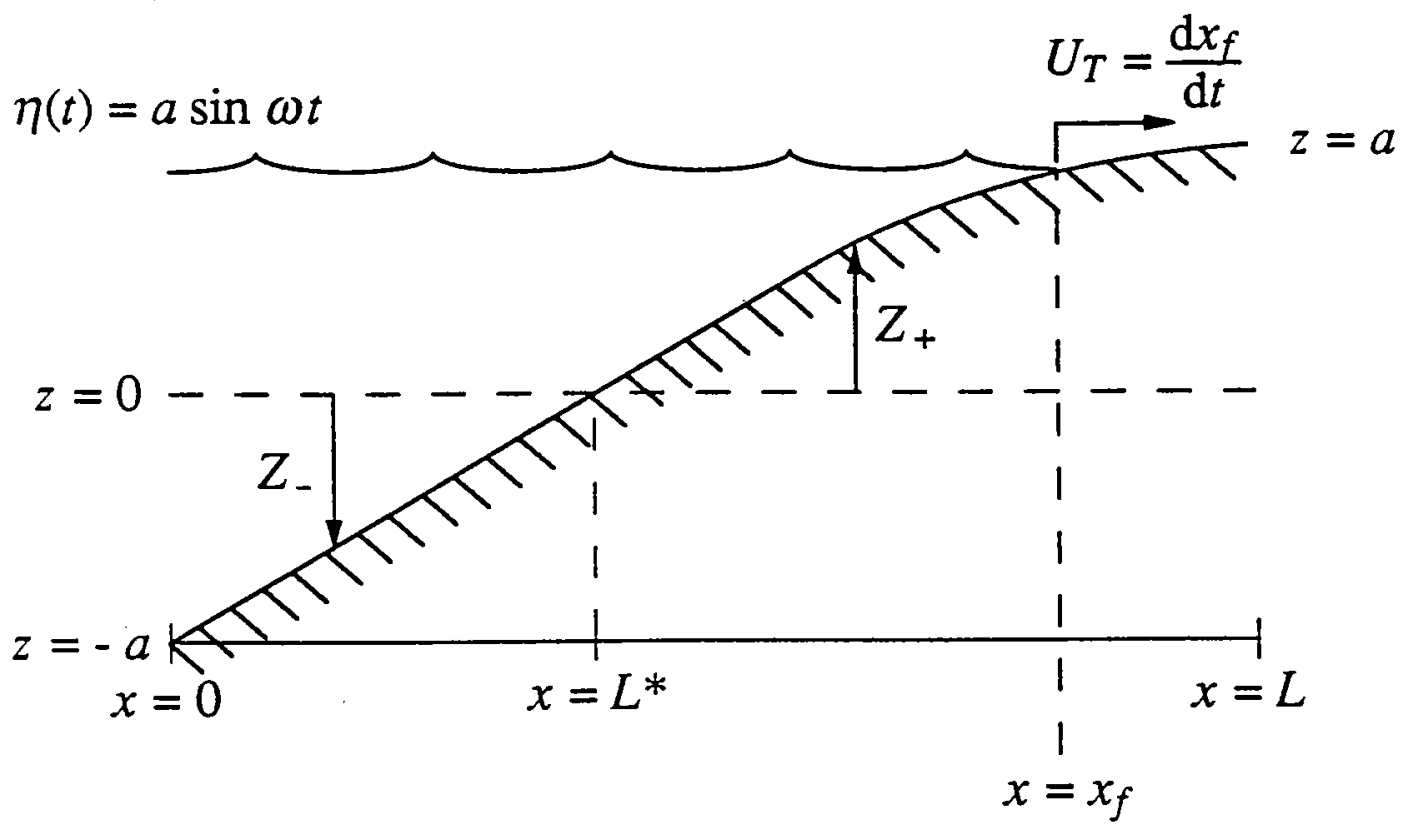

Figure 5.4 
Profile with $U_{T}$ constant:

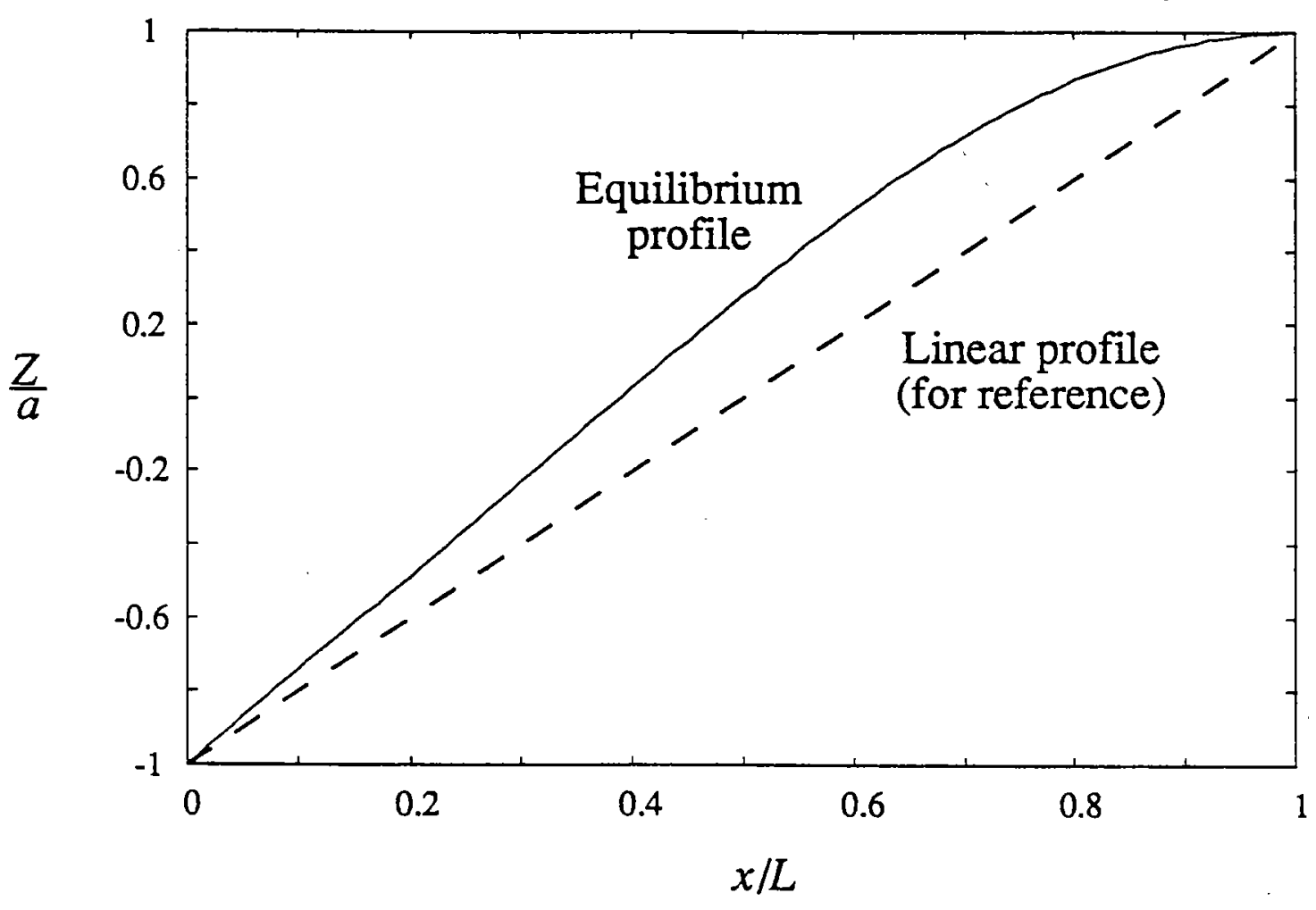

Figure 5.5 
Tidal flat along curved shoreline: Definitions

LOBATE shoreline:

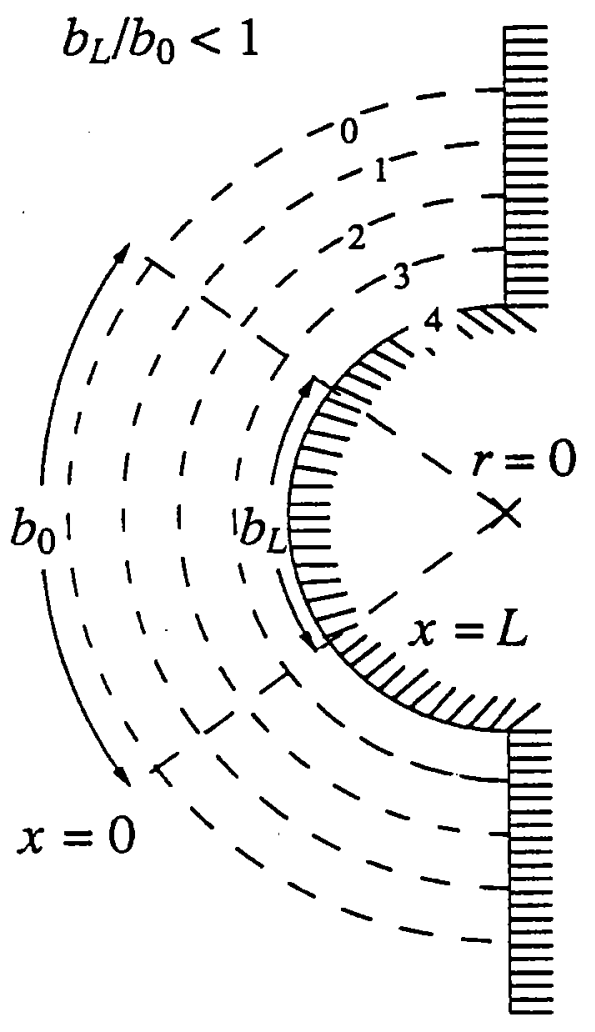

EMBAYED shoreline:

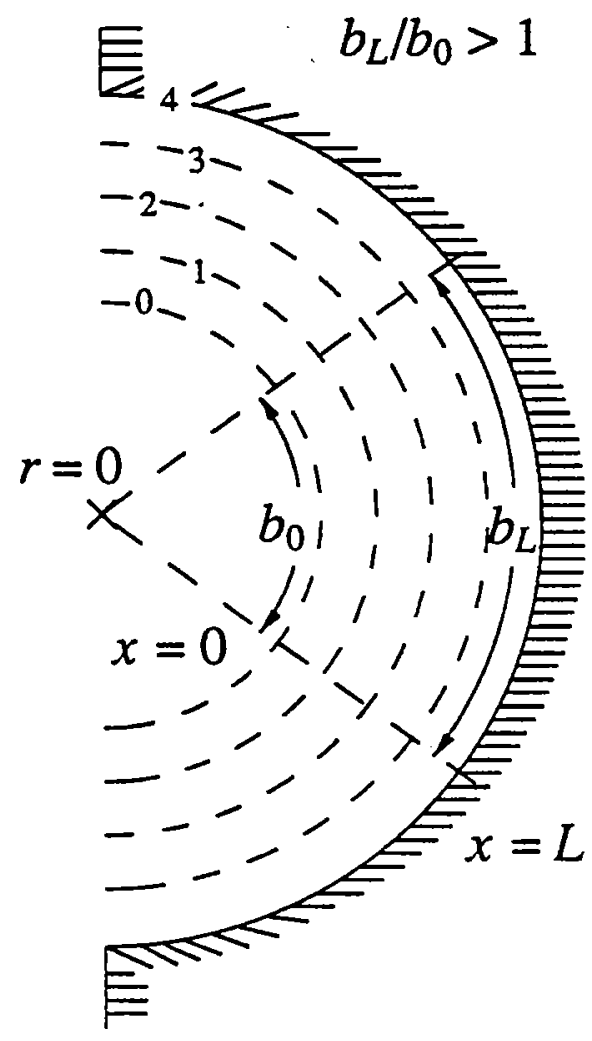

Figure 5.6 
Profiles along curved shorelines with $U_{T}$ constant:

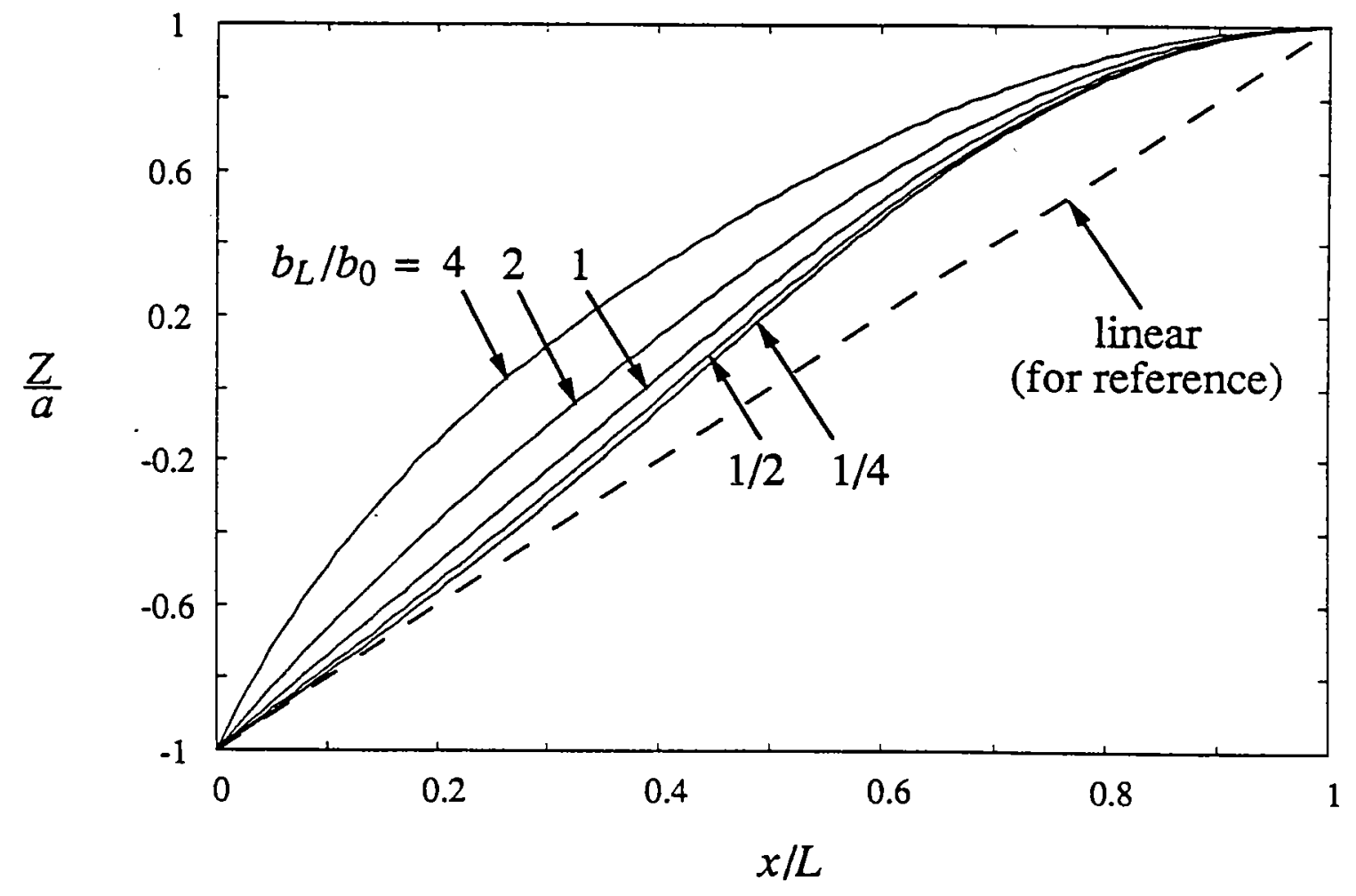

Figure 5.7 
Hypsometries along curved shorelines with $U_{T}$ constant:

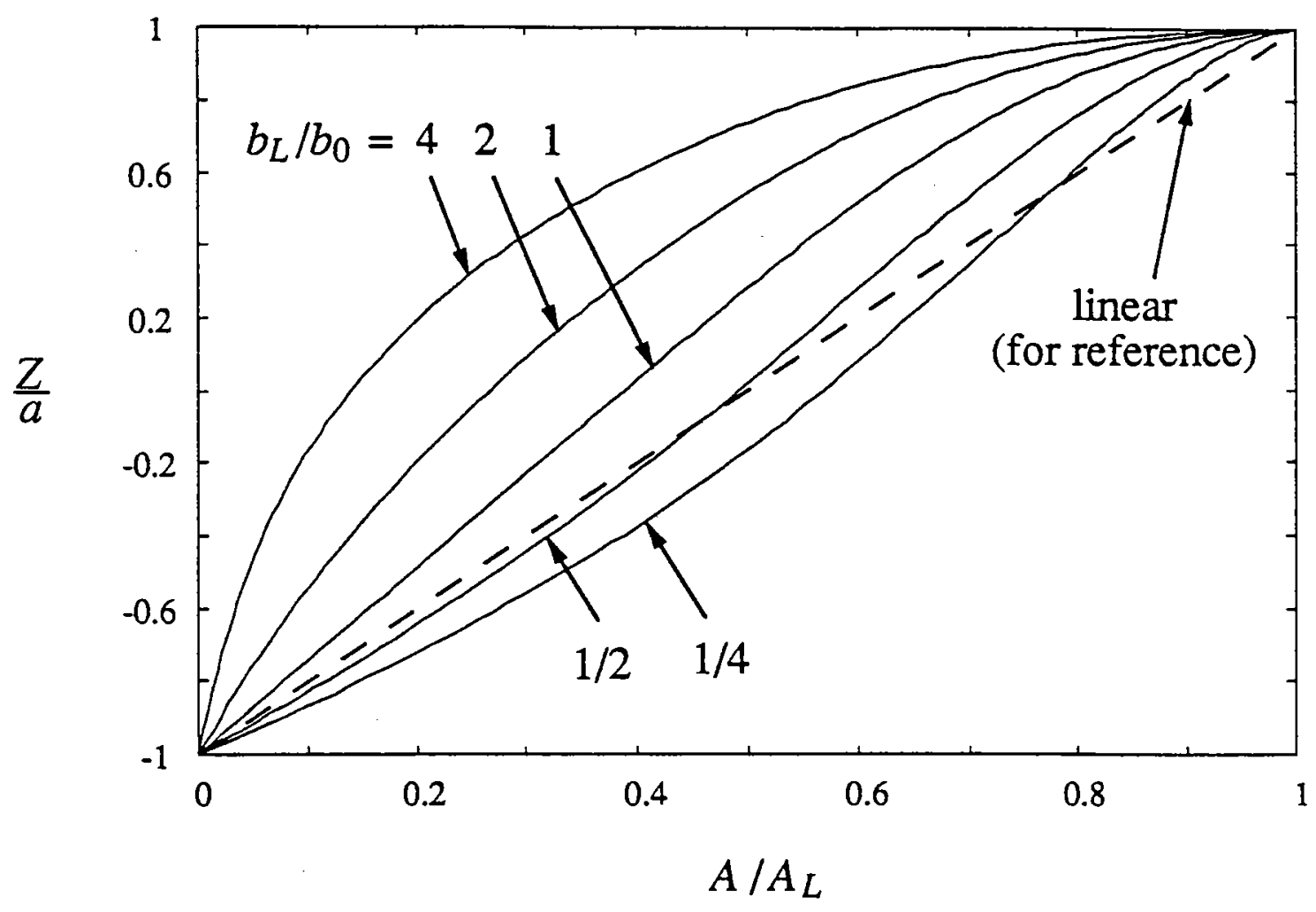

Figure 5.8 
Wind wave dominated linear flat profile: Definitions

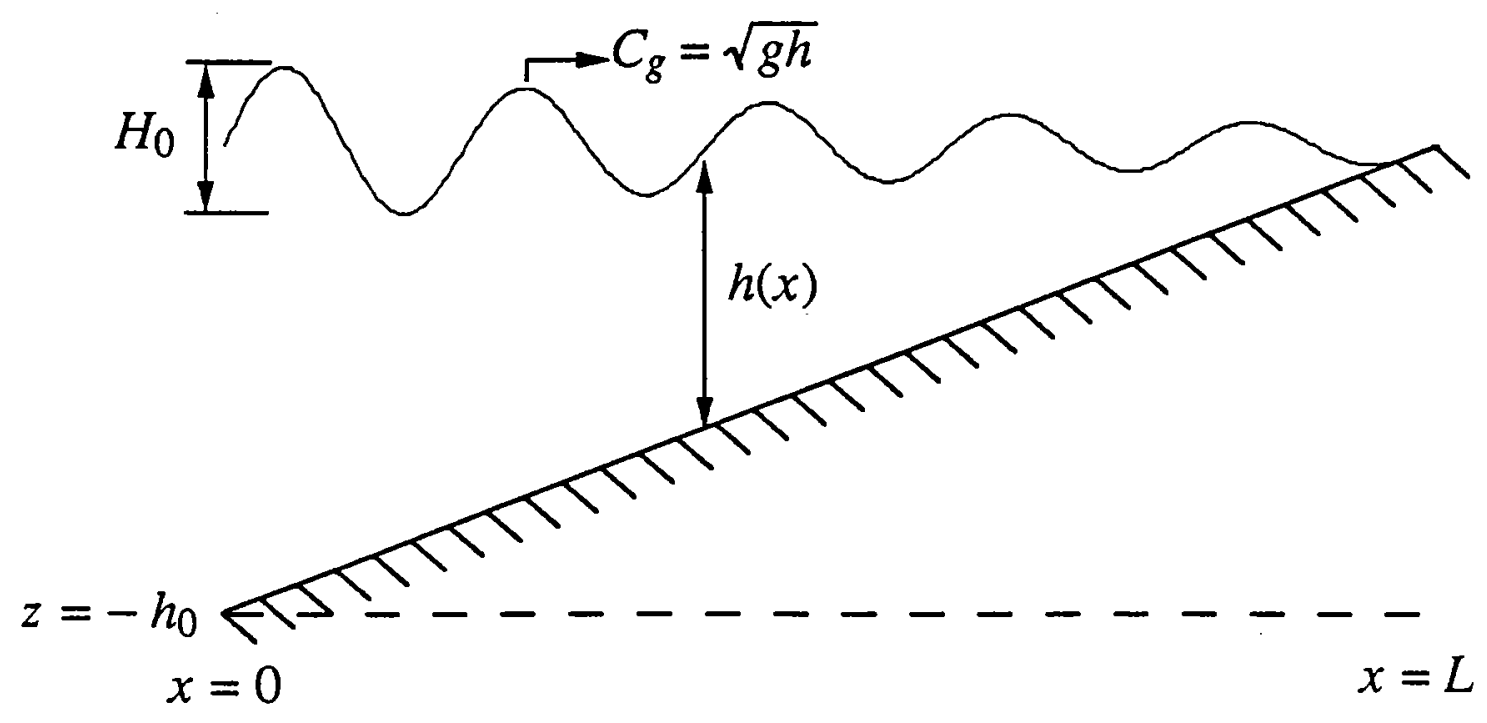

Figure 5.9 
$U_{W}$ and $\tau_{W}$ across a linearly sloping flat as a function of non-dimensional $H_{0}$ :
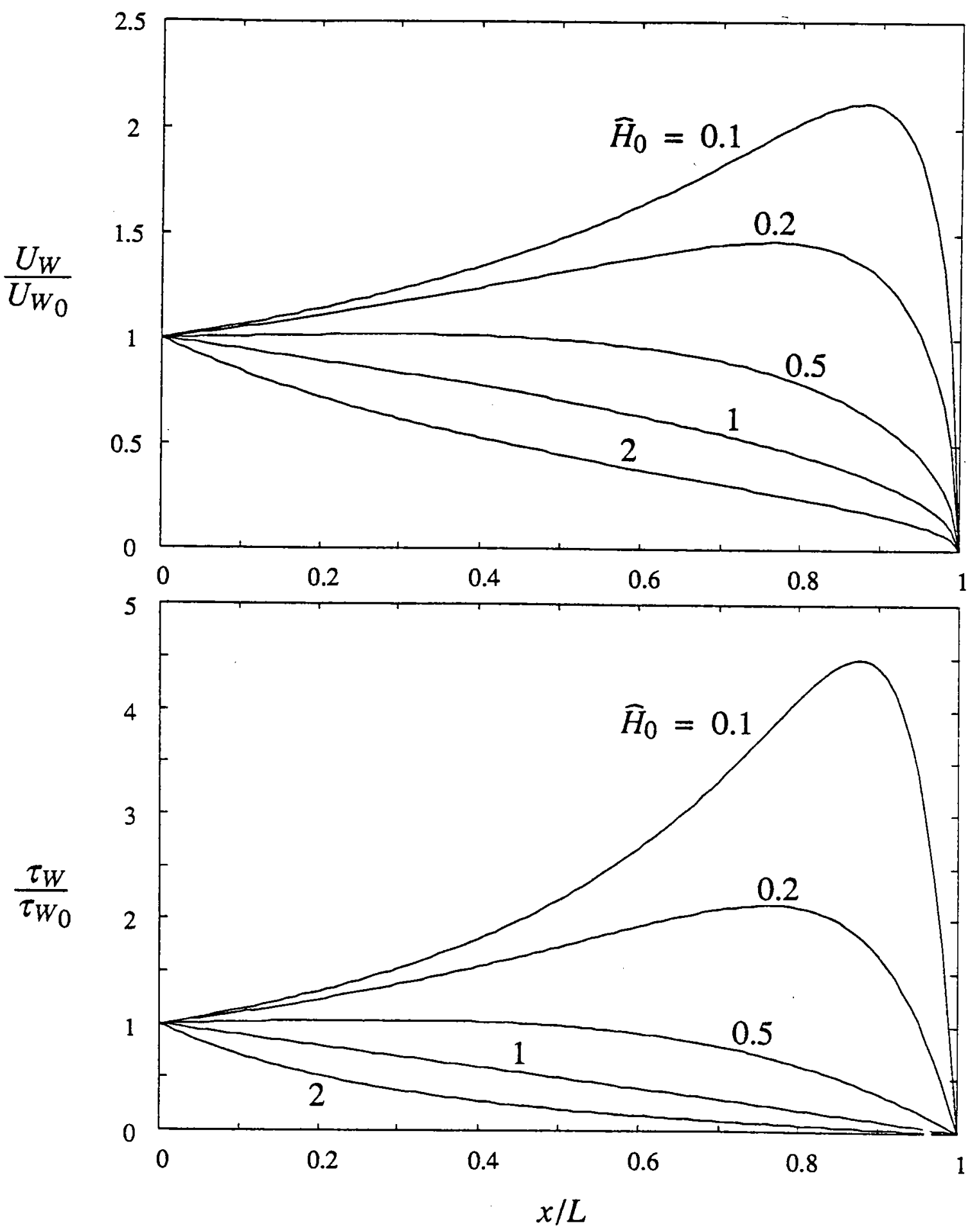

Figure 5.10 
Wind wave dominated non-linear flat profile: Definitions

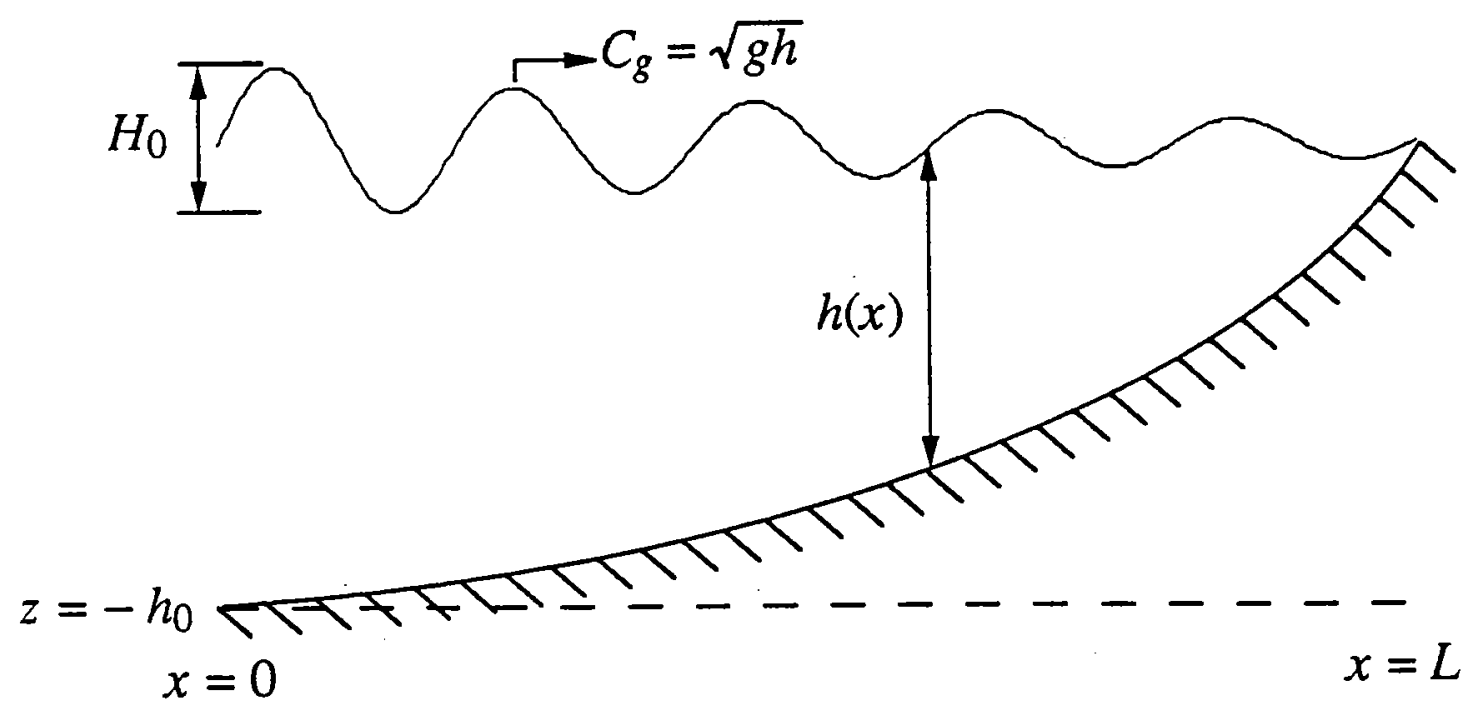

Figure 5.11 
Profile with $U_{W}$ constant:

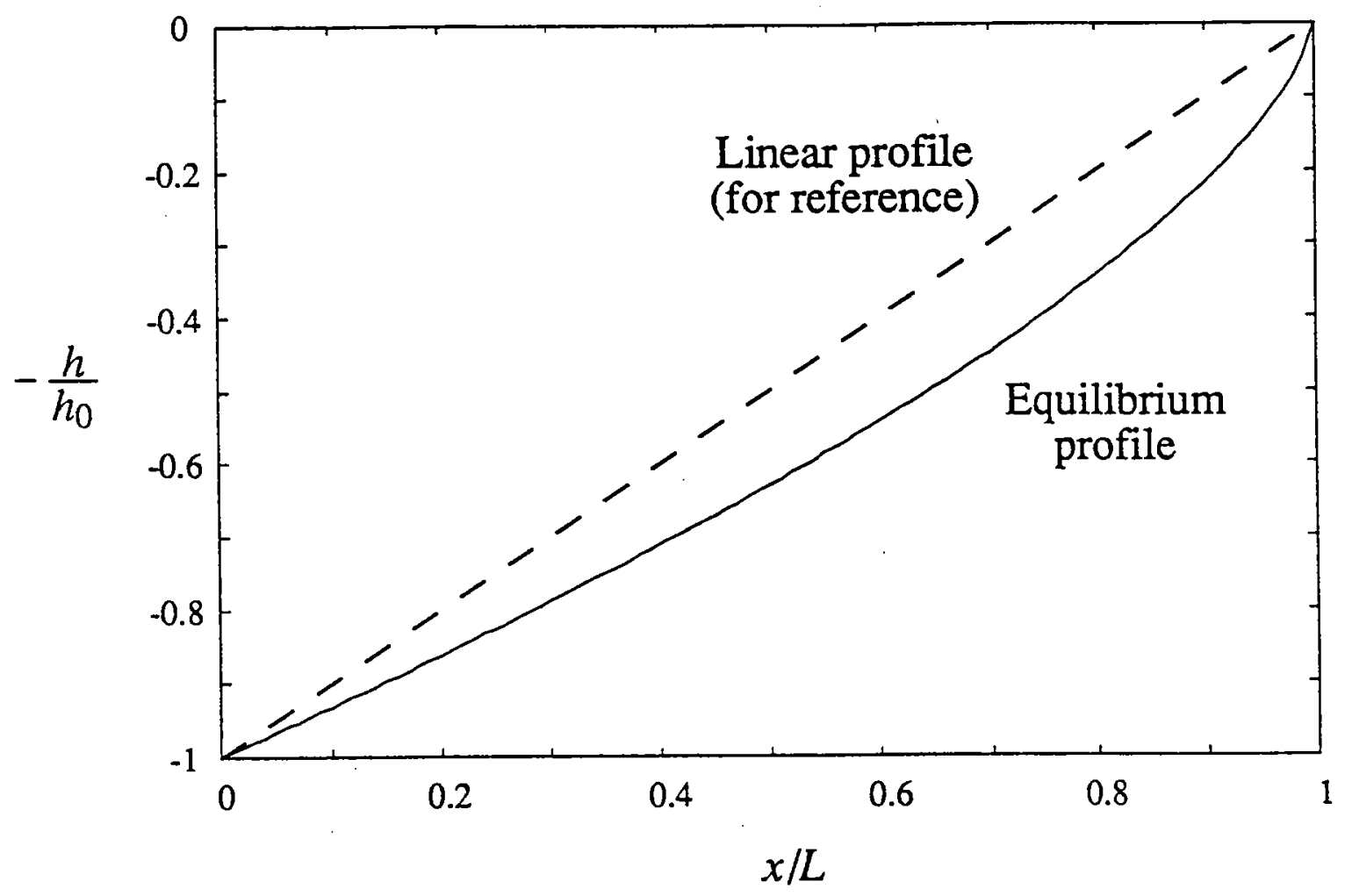

Figure 5.12 
Profiles along curved shorelines with $U_{W}$ constant:

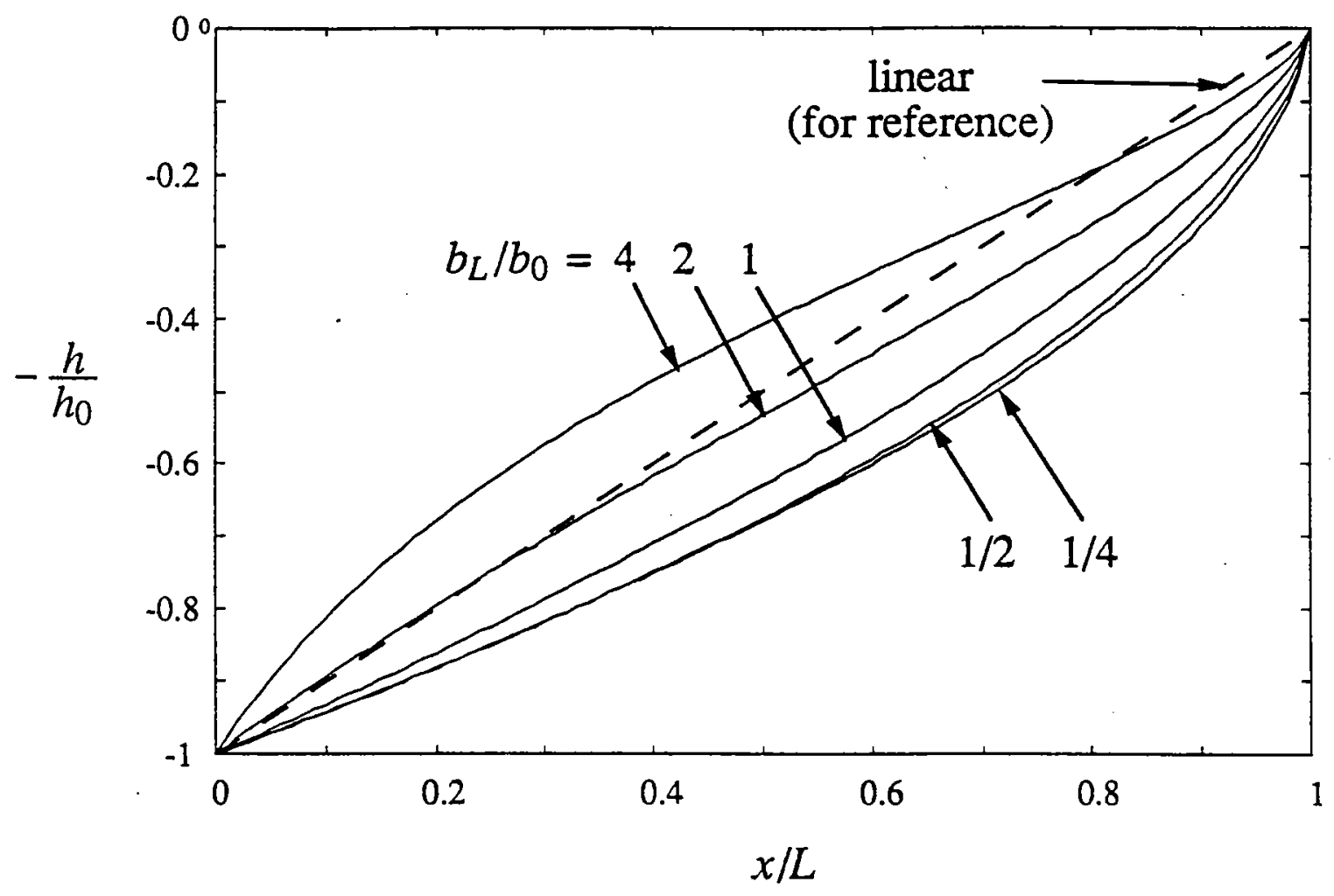

Figure 5.13 
Hypsometries along curved shorelines with $U_{W}$ constant:

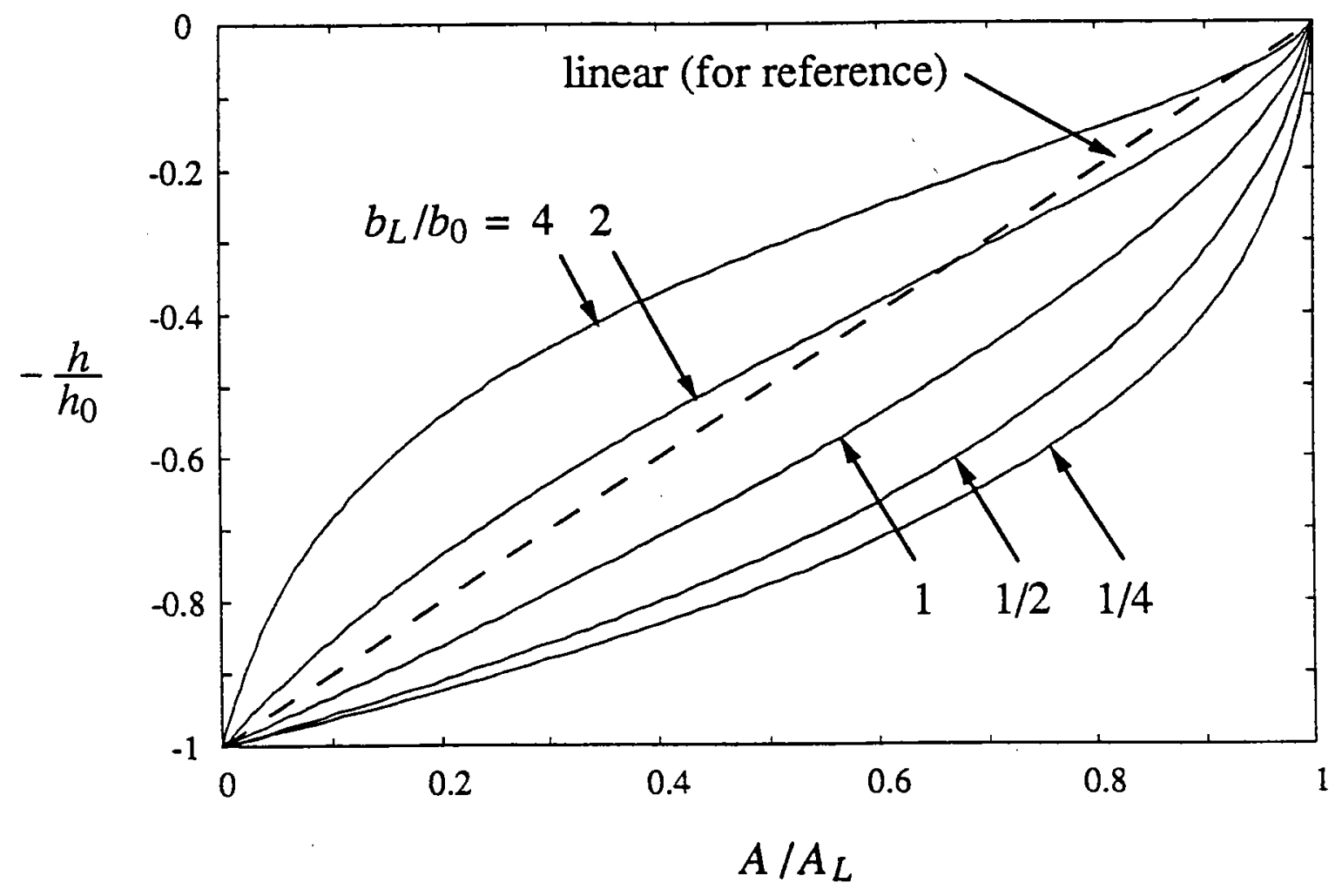

Figure 5.14 
$-194-$

Appendices 
Appendix 1: Solution of the zero-inertia diffusion equation for embayments with variations in along-channel geometry

Here we extend the derivation presented in Chapter 2 by no longer assuming $\bar{b}, w$ and $\bar{h}$ to be constant in $x$. Allowing along-channel variations in $\bar{b}, w$ and $\bar{h}$ does not affect the time-varying portion of the second-order solution. Thus an identical transformation can be made to the time variable $\tau$, and we again look for solutions of the form

$$
\zeta_{m}(x, \tau)=a A_{m} \xi_{m}(x) \exp i\left(m \omega \tau+\varphi_{m}\right)
$$

The boundary conditions on $\zeta_{m}$ are also the same as in Chapter 2, namely,

$$
\zeta_{m}=a A_{m} \exp i\left(m \omega \tau+\varphi_{m}\right) \quad \text { at } \quad x=L
$$

and, using Equation (2.7b),

$$
u_{m} \sim \bar{h}^{2 / 3} \frac{\partial \zeta_{m}}{\partial x}=0 \quad \text { at } \quad x=0
$$

Thus the boundary conditions on $\xi_{m}$ are

$$
\xi_{m}=1 \quad \text { at } \quad x=L, \quad \text { and } \quad \bar{h}^{2 / 3} \frac{\mathrm{d} \xi_{m}}{\mathrm{~d} x}=0 \quad \text { at } \quad x=0 .
$$

If we do not assume $\bar{b}, w$ and $\bar{h}$ to be constant in $x$, then Equation (2.42) becomes

$$
i m \omega \xi_{m}-D_{0} \frac{b_{0, L}}{\bar{b}(x)} \frac{\mathrm{d}}{\mathrm{d} x}\left\{\frac{w(x)}{w_{0, L}}\left(\frac{\bar{h}(x)}{h_{0, L}}\right)^{5 / 3} \frac{\mathrm{d} \xi_{m}}{\mathrm{~d} x}\right\}=0 .
$$

where the subscripts 0 or $L$ signify values at $x=0$ or $x=L$ (depending on the formulation of $\bar{b}, w$ and $\bar{h}$ ), and $b_{0, L}, w_{0, L}$ and $h_{0, L}$ replace $\bar{b}, w$ and $\bar{h}$ in $D_{0}$. In Appendix 1.1 we solve for $\xi_{m}$ assuming a power-law variation in the along-channel cross-section, whereas in Appendix 1.2 we assume the channel to vary exponentially.

\section{A 1.1. Power-law variation}

If the along channel cross-section is assumed to vary as follows,

$$
\bar{b}(x)=b_{L}\left(\frac{x}{L}\right)^{\sigma_{b}}, w(x)=w_{L}\left(\frac{x}{L}\right)^{\sigma_{w}}, \bar{h}(x)=h_{L}\left(\frac{x}{L}\right)^{\sigma_{h}},
$$

then (A 1.5) becomes: 


$$
\xi_{m}-\left(\frac{x}{L}\right)^{-\sigma_{b}} k_{m}^{-2} \frac{\mathrm{d}}{\mathrm{d} x}\left\{\left(\frac{x}{L}\right)^{5 / \sigma_{h}+\sigma_{w}} \frac{\mathrm{d} \xi_{m}}{\mathrm{~d} x}\right\}=0
$$

where $k_{m}^{2}=i m \omega / D_{0}$. Equation (A1.6) can be solved through the use of Bessel functions. We define

$$
\widehat{x}=\frac{i k_{m} L}{\sigma}\left(\frac{x}{L}\right)^{\sigma}, \text { with } \sigma=1-\frac{1}{2}\left(-\sigma_{b}+5 / 3 \sigma_{h}+\sigma_{w}\right)
$$

Then

$$
\frac{\mathrm{d}}{\mathrm{d} x}=i k_{m}\left(\frac{x}{L}\right)^{\sigma-1} \frac{\mathrm{d}}{\mathrm{d} \widehat{x}}
$$

and (A1.7) becomes

$$
\xi_{m}+\left(\frac{x}{L}\right)^{-\hat{\sigma}} \frac{\mathrm{d}}{\mathrm{d} x}\left\{\left(\frac{x}{L}\right)^{\hat{\sigma}} \frac{\mathrm{d} \xi_{m}}{\mathrm{~d} x}\right\}=0, \text { with } \hat{\sigma}=\frac{1}{2}\left(\sigma_{b}+5 / 3 \sigma_{h}+\sigma_{w}\right) \text {. (A1.10a,b) }
$$

Or, if we eliminate $x$ altogether:

$$
\left.\xi_{m}+\hat{x}^{-\hat{\sigma} / \sigma} \frac{\mathrm{d}}{\mathrm{d} \hat{x}} \mid \hat{x} \hat{\sigma} / \sigma \frac{\mathrm{d} \xi_{m}}{\mathrm{~d} \hat{x}}\right\}=0
$$

Next we let

$$
\hat{\xi}=\hat{x}^{v} \xi_{m}, \text { with } \quad v=\frac{1}{2}\left(\frac{\hat{\sigma}}{\sigma}-1\right)
$$

Then after some algebra and differentiation, (A1.11) becomes

$$
\frac{\mathrm{d}^{2} \hat{\xi}}{\mathrm{d} \widehat{x}^{2}}+\frac{1}{\hat{x}} \frac{\mathrm{d} \hat{\xi}}{\mathrm{d} \widehat{x}}+\left(1-\frac{v^{2}}{\widehat{x}^{2}}\right) \hat{\xi}=0
$$

i.e., a Bessel equation of order $v$ which, assuming $v$ is not exactly an integer, has the following solution (e.g., Boas 1983):

$$
\hat{\xi}=C_{1} \mathrm{~J}_{v}(\hat{x})+C_{2} \mathrm{~J}_{-v}(\hat{x})
$$

$\mathrm{J}_{ \pm v}$ is a Bessel function of the first kind, whose value is given by the infinite series

$$
\mathrm{J}_{ \pm v}(\hat{x})=\sum_{n=0}^{\infty} \frac{(-1)^{n}}{\Gamma(n+1) \Gamma(n \pm v+1)}\left(\frac{\hat{x}}{2}\right)^{2 n \pm v} .
$$

Utilizing (A1.12a), (A1.14) can finally be re-expressed as a general solution for $\xi_{m}$ : 


$$
\xi_{m}=\hat{x}^{-\mu}\left(C_{1} \mathrm{~J}_{\mathfrak{v}}(\hat{x})+C_{2} \mathrm{~J}_{-v}(\hat{x})\right) .
$$

The boundary condition at $x=0$ requires $u=0$. From (A1.4b) and (A1.6c), this condition is equivalent to $x^{2 / 3} \sigma h \mathrm{~d} \xi_{m} / \mathrm{d} x=0$. Substituting (A1.16) into (A1.9), this boundary condition may be re-expressed as

$$
x^{2 \beta} \sigma_{h}+\sigma-1 \frac{\mathrm{d}}{\mathrm{d} \widehat{x}}\left\{\hat{x}^{-v}\left(C_{1} \mathrm{~J}_{v}+C_{2} \mathrm{~J}_{-v}\right)\right\}=0 \quad \text { at } \quad x=0 .
$$

After differentiating, using (A1.8a) to eliminate $x$, and assuming $\sigma>0$ (so that $\hat{x} \rightarrow 0$ when $x \rightarrow 0$ ), (A1.17) becomes

$$
\hat{x}-\tilde{\sigma}-v\left\{C_{1}\left(-v \mathrm{~J}_{v}+\hat{x} \frac{\mathrm{d} \mathrm{J}_{v}}{\mathrm{~d} \hat{x}}\right)+C_{2}\left(-v \mathrm{~J}_{-v}+\hat{x} \frac{\mathrm{dJ}-v}{\mathrm{~d} \hat{x}}\right)\right\}=0 \text { at } \hat{x}=0,
$$

where $\tilde{\sigma}=\left(1-2 / 3 \sigma_{h}\right) / \sigma$. Using the following Bessel identities (e.g., Boas 1983),

$$
\widehat{x} \frac{d J_{ \pm v}}{\mathrm{~d} \hat{x}}= \pm v \mathrm{~J}_{ \pm v}-\widehat{x} \mathrm{~J}_{ \pm v+1}=(-1)( \pm v) \mathrm{J}_{ \pm v}+\widehat{x} \mathrm{~J}_{ \pm v-1},
$$

(A1.18) is equivalent to

$$
\hat{x}^{-\tilde{\sigma}-v+1}\left(C_{1} \mathrm{~J}_{v+1}(\hat{x})-C_{2} \mathrm{~J}_{-v-1}(\hat{x})\right)=0 \text { at } \hat{x}=0 .
$$

As $\hat{x} \rightarrow 0$, only the first term of (A1.15) need be retained, and $J_{ \pm v} \rightarrow(\hat{x} / 2)^{ \pm v} / \Gamma( \pm v+1)$. Equation (A1.20) then asymptotes to

$$
\left(\frac{1}{2}\right)^{v+1} \frac{C_{1}}{\Gamma(v+2)} \hat{x}^{-\tilde{\sigma}+2}-\left(\frac{1}{2}\right)^{-v-1} \frac{C_{2}}{\Gamma(-v)} \hat{x}^{-\tilde{\sigma}-2 v} \rightarrow 0 \quad \text { as } \quad \hat{x} \rightarrow 0 .
$$

If $\tilde{\sigma}<2$ and $2 v+\tilde{\sigma} \geq 0$, (A1.21) will go to zero only if $C_{2}=0$. With $C_{2}=0$, (A1.16) gives $\xi_{m}=\hat{x}^{-v} C_{1} \mathrm{~J}_{v}$, and application of the second boundary condition, i.e., $\xi_{m}=$ 1 at $x=L$, finally gives the following solution for $\xi_{m}$ :

$$
\xi_{m}=\left(\frac{\hat{x}}{\hat{L}}\right)^{-v} \frac{\mathrm{J}_{V}(\hat{x})}{\mathrm{J}_{V}(\hat{L})},
$$

where $\widehat{L}=\widehat{x}(x=L)$. For a prismatic channel, $\sigma_{b}=\sigma_{h}=\sigma_{w}=\widehat{\sigma}=0, \sigma=\tilde{\sigma}=1$, and $v=-1 / 2$. Then if we utilize the Bessel identity (e.g., Boas 1983)

$$
\mathrm{J}_{-1 / 2}(\hat{x})=\left(\frac{2}{\pi}\right)^{1 / 2} \hat{x}^{-1 / 2} \cosh (\hat{x} / i),
$$


(A 1.22) reverts directly back to Equation (2.44).

\section{A 1.2. Exponential variation}

We now assume an exponential variation in the along channel cross-section, i.e., we let

$$
\bar{b}(x)=b_{0} \mathrm{e}^{\lambda_{b} x / L}, w(x)=w_{0} \mathrm{e}^{\lambda_{w} x / L}, \bar{h}(x)=h_{0} \mathrm{e}^{\lambda_{h} x / L} .
$$

Then (A1.5) becomes:

$$
\xi_{m}-\mathrm{e}^{-\lambda_{b} x / L} k_{m}^{-2} \frac{\mathrm{d}}{\mathrm{d} x}\left\{\mathrm{e}^{\left(\lambda_{w}+5 / 3 \lambda_{h}\right) x / L} \frac{\mathrm{d} \xi_{m}}{\mathrm{~d} x}\right\}=0
$$

with $k_{m}{ }^{2}=i m \omega / D_{0}$. Equation (A1.25), like (A1.7), can be solved via Bessel functions, and we proceed in a manner similar to that followed in Appendix 1.1. First we let

$$
\begin{gathered}
\hat{x}=-\frac{i k_{m} L}{\lambda} \mathrm{e}^{-\lambda x / L}, \quad \text { with } \quad \lambda=\frac{1}{2}\left(-\lambda_{b}+5 / 3 \lambda_{h}+\lambda_{w}\right), \\
\hat{\lambda}=\frac{1}{2}\left(\lambda_{b}+5 / 3 \lambda_{h}+\lambda_{w}\right), \quad \hat{\xi}=\hat{x}^{v} \xi_{m}, \quad \text { and } \quad v=\frac{1}{2}\left(\frac{\hat{\lambda}}{\lambda}-1\right) .
\end{gathered}
$$

Then after some algebra and differentiation, (A1.25) becomes a Bessel equation of order $v$ which has a general solution of the form

$$
\hat{\xi}=C_{1} \mathrm{~J}_{v}(\hat{x})+C_{2} \mathrm{~J}_{-v}(\hat{x}), \quad \text { or } \quad \xi=\hat{x}^{-v}\left(C_{1} \mathrm{~J}_{v}+C_{2} \mathrm{~J}_{-v}\right) . \quad(\mathrm{A} 1.27 \mathrm{a}, \mathrm{b})
$$

The boundary condition $\mathrm{d} \xi_{m} / \mathrm{d} x=0$ at $x=0$ may be re-expressed as

$$
\frac{\mathrm{d}}{\mathrm{d} \hat{x}}\left\{\hat{x}^{-v}\left(C_{1} \mathrm{~J}_{v}+C_{2} \mathrm{~J}_{-v}\right)\right\}=0 \text { at } \hat{x}=\hat{x}_{0}
$$

where $\widehat{x}_{0}=i k L_{m} / \lambda$. After differentiating (A1.28) and employing the identities in (A1.19), (A1.28) becomes equivalent to

$$
C_{1} \mathrm{~J}_{v+1}\left(\hat{x}_{0}\right)=C_{2} \mathrm{~J}_{-v-1}\left(\hat{x}_{0}\right)=C_{3} \mathrm{~J}_{v+1}\left(\hat{x}_{0}\right) \mathrm{J}_{-v-1}\left(\hat{x}_{0}\right)
$$

where $C_{3}$ is a new constant. Using (A1.29) to eliminate $C_{1}$ and $C_{2}$ in (A1.27b) then gives

$$
\xi_{m}=\hat{x}^{-v} C_{3}\left(\mathrm{~J}_{\mu}(\hat{x}) \mathrm{J}_{-v-1}\left(\hat{x}_{0}\right)-\mathrm{J}_{-v}(\hat{x}) \mathrm{J}_{v+1}\left(\hat{x}_{0}\right)\right)=0
$$


Application of the second boundary condition, i.e., $\xi_{m}=1$ at $x=\dot{L}$, finally gives the following solution for $\xi_{m}$ :

$$
\xi_{m}=\left(\frac{\hat{x}}{\hat{L}}\right)^{-v} \frac{\mathrm{J}_{v}(\hat{x}) \mathrm{J}_{-v-1}\left(\hat{x}_{0}\right)+\mathrm{J}_{-v}(\hat{x}) \mathrm{J}_{v+1}\left(\hat{x}_{0}\right)}{\mathrm{J}_{v}(\hat{L}) \mathrm{J}_{-v-1}\left(\hat{x}_{0}\right)+\mathrm{J}_{-v}(\hat{L}) \mathrm{J}_{v+1}\left(\hat{x}_{0}\right)},
$$

where $\hat{L}=\hat{x}(x=L)$. For a prismatic channel, $\lambda_{b}=\lambda_{h}=\lambda_{w}=\lambda=\hat{\sigma}=0$, and $v=-1 / 2$. Using (A1.23) and the additional Bessel identity (e.g., Boas 1983),

$$
\mathrm{J}_{1 / 2}(\hat{x})=i\left(\frac{2}{\pi}\right)^{1 / 2} \hat{x}^{-1 / 2} \sinh (\hat{x} / i)
$$

(A1.31) becomes

$$
\xi_{m}=\frac{\cosh \hat{x} / i \cosh \hat{x}_{0} / i-\sinh \hat{x} / i \sinh \hat{x}_{0} / i}{\cosh \hat{L} / i \cosh \hat{x}_{0} / i-\sinh \hat{L} / i \sinh \hat{x}_{0} / i}=\frac{\cosh \left(\hat{x}-\hat{x}_{0}\right) / i}{\cosh \left(\widehat{L}-\hat{x}_{0}\right) / i}
$$

Equation (A1.33) can be transformed back to Equation (2.44) by using (A1.26a) to eliminate $\hat{x}$ and then employing the asymptote $\mathrm{e}^{-\lambda x / L} \approx-(1-\lambda x / L)$ as $\lambda \rightarrow 0$. 
Appendix 2. Transformation of the time domain in the second-order zero-inertia equation

We are given that

$$
\omega \tau=\omega t+\gamma_{1} \sin \left(\omega t-\theta_{1}\right)+\gamma_{2} \sin \left(2 \omega t-\theta_{2}\right)
$$

where $O(\omega \tau)=O(\omega t)=1$, and $O\left(\eta_{1}\right)=O\left(\gamma_{2}\right)=O(\gamma) \ll 1$. In Appendix 2.1 it is shown that to $\mathrm{O}(\gamma)$,

$$
\cos \omega t=\sum_{m=-1}^{3} Z_{m} \cos \left(m \omega \tau-\varphi_{m}\right)
$$

where $Z_{-1}=-Z_{3}=\gamma_{2} / 2, Z_{0}=-Z_{2}=\gamma_{1} / 2, Z_{1}=1,-\varphi_{-1}=\varphi_{3}=\theta_{2},-\varphi_{0}=\varphi_{2}=\theta_{1}$, and $\varphi_{1}=$ 0 . In Appendix 2.2 it is shown that to $\mathrm{O}(\gamma)$,

$$
\exp i \omega \tau=\exp i \omega t-\sum_{m \neq 1} Z_{m} \exp i\left(m \omega t-\varphi_{m}\right)
$$

A2.1. Transformation from $t$ to $\tau$

Equation (A2.1) can be rewritten as

$$
\cos \omega t=\cos \left\{\omega \tau-\gamma_{1} \sin \left(\omega t-\theta_{1}\right)-\gamma_{2} \sin \left(2 \omega t-\theta_{2}\right)\right\},
$$

which to $O(\gamma)$ is equivalent to

$$
\cos \omega t=\cos \left\{\omega \tau-\gamma_{1} \sin \left(\omega \tau-\theta_{1}\right)-\gamma_{2} \sin \left(2 \omega \tau-\theta_{2}\right)\right\}
$$

Using the identity $\cos (\alpha-\beta)=\cos \alpha \cos \beta+\sin \alpha \sin \beta$, (A2.5) becomes

$$
\cos \omega t=\cos \omega \tau \cos \varepsilon+\sin \omega \tau \sin \varepsilon,
$$

where

$$
\varepsilon=\gamma_{1} \sin \left(\omega \tau-\theta_{1}\right)+\gamma_{2} \sin \left(2 \omega \tau-\theta_{2}\right)=O(\gamma) .
$$

To $\mathrm{O}(\gamma),(\mathrm{A} 2.6)$ can te rewritten as

$$
\cos \omega t=\cos \omega \tau+\varepsilon \sin \omega \tau \text {. }
$$

The second term on the r.h.s. of (A2.8) can then be expanded as 


$$
\varepsilon \sin \omega \tau=\eta_{1} \sin \omega \tau \sin \left(\omega \tau-\theta_{1}\right)+\gamma_{2} \sin \omega \tau \sin \left(2 \omega \tau-\theta_{2}\right),
$$

and use of the identity $\sin \alpha \sin \beta=1 / 2\{\cos (\alpha-\beta)-\cos (\alpha+\beta)\}$ produces

$$
\varepsilon \sin \omega \tau=\frac{\gamma_{1}}{2}\left\{\cos \theta_{1}-\cos \left(2 \omega \tau-\theta_{1}\right)\right\}+\frac{\gamma_{2}}{2}\left\{\cos \left(-\omega \tau+\theta_{2}\right)-\cos \left(3 \omega \tau-\theta_{2}\right)\right\} .
$$

Finally, substitution of (A2.10) into (A2.6) gives (A2.2).

\section{A2.2. Transformation from $\tau$ to $t$}

Equation (A2.1) may also be rewritten to $O(\gamma)$ as

$$
\sin \omega t=\sin \left\{\omega \tau-\gamma_{1} \sin \left(\omega \tau-\theta_{1}\right)-\gamma_{2} \sin \left(2 \omega \tau-\theta_{2}\right)\right\} .
$$

Then using the identity $\sin (\alpha-\beta)=\sin \alpha \cos \beta-\cos \alpha \sin \beta$, (A2.11) becomes, to $\mathrm{O}(\gamma)$,

$$
\sin \omega t=\sin \omega \tau-\varepsilon \cos \omega \tau .
$$

The second term on the r.h.s. of (A2.12) can then be expanded as

$$
\varepsilon \cos \omega \tau=\gamma_{1} \cos \omega \tau \sin \left(\omega \tau-\theta_{1}\right)+\gamma_{2} \cos \omega \tau \sin \left(2 \omega \tau-\theta_{2}\right),
$$

and use of the identity $\cos \alpha \sin \beta=1 / 2\{\sin (\alpha+\beta)-\sin (\alpha-\beta)\}$ produces

$$
\varepsilon \cos \omega \tau=\frac{\gamma_{1}}{2}\left\{\sin \left(2 \omega \tau-\theta_{1}\right)-\sin \theta_{1}\right\}+\frac{\gamma_{2}}{2}\left\{\sin \left(3 \omega \tau-\theta_{2}\right)-\sin \left(-\omega \tau+\theta_{2}\right)\right\} .
$$

Finally, substitution of (A2.14) into (A2.12) gives

$$
\sin \omega t=\sum_{m=-1}^{3} Z_{m} \sin \left(m \omega \tau-\varphi_{m}\right)
$$

Combination of (A2.2) and (A2.15) gives

$$
\exp i \omega t=\cos \omega t+i \sin \omega t=\sum_{m=-1}^{3} Z_{m} \exp i\left(m \omega \tau-\varphi_{m}\right),
$$

or, solving for $\exp i \omega \tau$,

$$
\exp i \omega \tau=\exp i \omega t-\sum_{m \neq 1} Z_{m} \exp i\left(m \omega \tau-\varphi_{m}\right) .
$$

To $O(\gamma),(A 2.17)$ is equivalent to (A2.3). 
Appendix 3. The relative phase of the second-harmonic in strongly convergent channels near morphologic equilibrium

Here relations for the relative phase of the second harmonic are derived for both tidal elevation and velocity. The phase of $\eta_{2}$ relative to $\eta_{1}$ is derived in Appendix 3.1, and the phase of $V_{2}$ relative to $V_{1}$ is derived in Appendix 3.2.

\section{A3.1. $\eta_{2}$ to $\eta_{1}$ relative phase}

From Equation (4.4.19), the non-dimensionalized second-harmonic for tidal elevation is given by

$$
\eta_{2}=\frac{\gamma}{2} \mathrm{e}^{i 2 \omega t}\left\{\mathrm{e}^{\mu k x} \mathrm{e}^{-i\left(k x+k x_{0}\right)}-\mathrm{e}^{2 \mu k x} \mathrm{e}^{-i\left(2 k x+k x_{0}\right)}\right\} .
$$

If we apply the identity $\mathrm{e}^{i x}=\cos x+i \sin x$, and separate $\gamma$ into its magnitude and argument, then (A3.1) becomes

$$
\begin{aligned}
\eta_{2}=\frac{|\gamma|}{2} & \mathrm{e}^{\mu k x} \exp i\{2 \omega-\pi \delta(1,-\gamma /|\gamma|)\} \\
& \cdot\left\{\begin{array}{r}
\cos \left(k x+k x_{0}\right)-i \sin \left(k x+k x_{0}\right) \\
-\mathrm{e}^{\mu k x} \cos \left(2 k x+k x_{0}\right)+i \mathrm{e}^{\mu k x} \sin \left(2 k x+k x_{0}\right)
\end{array}\right\},
\end{aligned}
$$

where $\delta(m, l)=1$ if $m=l, \delta=0$ otherwise. The identity $\arg (x+i y)=\exp i\{\arctan (y / x)\}$, is then used to derive the argument of (A3.2):

$$
\arg \left(\eta_{2}\right)=2 \omega-\pi \delta\left(1, \frac{-\gamma}{|\gamma|}\right)+\arctan \left\{\frac{\mathrm{e}^{\mu k x} \sin \left(2 k x+k x_{0}\right)-\sin \left(k x+k x_{0}\right)}{\cos \left(k x+k x_{0}\right)-\mathrm{e}^{\mu k x} \cos \left(2 k x+k x_{0}\right)}\right\} .
$$

The phase of $\eta_{2}=\left|\eta_{2}\right| \exp i\left(2 \omega t-\varphi_{\eta_{2}}\right)$ is then

$$
\varphi_{\eta 2}=\arctan \left\{\frac{\sin \left(k x+k x_{0}\right)-\mathrm{e}^{\mu k x} \sin \left(2 k x+k x_{0}\right)}{\cos \left(k x+k x_{0}\right)-\mathrm{e}^{\mu k x} \cos \left(2 k x+k x_{0}\right)}\right\}+\pi \delta\left(1, \frac{-\gamma}{|\gamma|}\right) \text {. }
$$

If we define $\alpha=3 / 2 k x+k x_{0}$ and $\beta=1 / 2 k x$, then if $\mu=0$, the identities $\sin (\alpha-\beta)$ $-\sin (\alpha+\beta)=-\cos \alpha \sin \beta$ and $\cos (\alpha-\beta)-\cos (\alpha+\beta)=\sin \alpha \sin \beta$ can be used to transform $(\mathrm{A} 3.4)$ to

$$
\begin{gathered}
\varphi_{\eta 2}=\arctan \left\{\frac{-\cos \left(3 / 2 k x+k x_{0}\right)}{\sin \left(3 / 2 k x+k x_{0}\right)}\right\}+\pi \delta\left(1, \frac{-\gamma}{|\gamma|}\right) \\
=\arctan \left\{\frac{-\sin \left(\pi / 2-3 / 2 k x-k x_{0}\right)}{\cos \left(\pi / 2-3 / 2 k x-k x_{0}\right)}\right\}+\pi \delta\left(1, \frac{-\gamma}{|\gamma|}\right) .
\end{gathered}
$$


Equation (A3.5) then reduces to

$$
\varphi_{\eta 2}=\frac{3}{2} k x+k x_{0}-\frac{\pi}{2} \frac{\gamma}{|\gamma|} .
$$

The phase of $\eta_{2}=\left|\eta_{2}\right| \exp i\left(2 \omega t-\varphi_{\eta 2}\right)$ relative to $\eta_{1}=\left|\eta_{1}\right| \exp i\left(\omega t-\varphi_{\eta 1}\right)$ is defined as $2 \varphi_{\eta 1}-\varphi_{\eta 2}$ (Aubrey and Speer 1985). So from (A3.6) for $\mu=0$,

$$
2 \varphi_{\eta 1}-\varphi_{\eta 2}=\frac{\pi}{2} \frac{\gamma}{|\gamma|}+\frac{k x}{2}-k x_{0}
$$

A3.2. $V_{2}$ to $V_{1}$ relative phase

From Equation (4.4.20), the non-dimensionalized second harmonic for tidal velocity is given by

$$
V_{2}=\frac{i \gamma}{2} \mathrm{e}^{i 2 \omega t}\left\{\mathrm{e}^{\mu k x} \mathrm{e}^{-i\left(k x+k x_{0}\right)}-2 \mathrm{e}^{2 \mu k x} \mathrm{e}^{-i\left(2 k x+k x_{0}\right)}\right\} .
$$

We will consider only the case of $\mu=0$. Following the same steps as in Appendix 3.1, (A3.8) can be rewritten as

$$
\begin{aligned}
V_{2}=\frac{|\gamma|}{2} & \exp i\{\pi / 2+2 \omega-\pi \delta(1,-\gamma /|\gamma|)\} \\
& \cdot\left\{\begin{array}{r}
\cos \left(k x+k x_{0}\right)-i \sin \left(k x+k x_{0}\right) \\
-2 \cos \left(2 k x+k x_{0}\right)+2 i \sin \left(2 k x+k x_{0}\right)
\end{array}\right\} .
\end{aligned}
$$

And the argument of (A3.9) is

$$
\arg \left(V_{2}\right)=2 \omega+\frac{\pi}{2} \frac{\gamma}{|\gamma|}+\arctan \left\{\frac{2 \sin \left(2 k x+k x_{0}\right)-\sin \left(k x+k x_{0}\right)}{\cos \left(k x+k x_{0}\right)-2 \cos \left(2 k x+k x_{0}\right)}\right\} .
$$

The phase of $V_{2}=\left|V_{2}\right| \exp i\left(2 \omega t-\varphi_{V}\right)$ is then

$$
\varphi_{V 2}=\arctan \left\{\frac{2 \sin \left(2 k x+k x_{0}\right)-\sin \left(k x+k x_{0}\right)}{2 \cos \left(2 k x+k x_{0}\right)-\cos \left(k x+k x_{0}\right)}\right\}-\frac{\pi}{2} \frac{\gamma}{|\gamma|} .
$$

From Equation (4.6.2), $\varphi_{V 1}=k x+\varepsilon_{T}-\pi / 2$. Therefore the phase of $V_{2}$ relative to $V_{1}$ for $\mu=0$ is given by

$$
2 \varphi_{V 1}-\varphi_{V 2}=2 k x+2 \varepsilon_{T}-\arctan \left\{\frac{2 \sin \left(2 k x+k x_{0}\right)-\sin \left(k x+k x_{0}\right)}{2 \cos \left(2 k x+k x_{0}\right)-\cos \left(k x+k x_{0}\right)}\right\}+\frac{\pi}{2} \frac{\gamma}{|\gamma|} .
$$


$-204-$

References 
Alexander, C. R., C. A. Nittrouer, D. J. Demaster, Y.-A. Park and S.-C. Park, 1991. Macrotidal mudflats of the southwestern Korean coast: a model for interpretation of intertidal deposits. Joumal of Sedimentary Petrology, 61: 805-824.

Allen, G. P., J. C. Salomon, P. Bassoullet, Y. Du Penhoat and C. De Grandpré, 1980. Effects of tides on mixing and suspended sediment transport in macrotidal estuaries. Sedimentary Geology, 26: 69-90.

Arons, A. B., and H. Stommel, 1951. A mixing-length theory of tidal flushing. Transactions, American Geophysical Union, 32: 419-421.

Aubrey, D. G., and P. E. Speer, 1984. Updrift migration of tidal inlets. Journal of Geology, 92: 531-545.

Aubrey, 1986. Hydrodynamic controls on sediment transport in well-mixed bays and estuaries. In: J. van de Kreeke (ed.), Physics of shallow estuaries and bays. Coastal and Estuarine Studies, Vol. 16, Springer-Verlag, New York, p. 245-258.

Aubrey, D. G., and P. E. Speer, 1985. A study of non-linear tidal propagation in shallow inlet/estuarine systems, part I: observations. Estuarine, Coastal and Shelf Science, 21: 185-205.

Bagnold, R. A., 1963. Beach and nearshore processes part I. mechanics of maine sedimentation. In: M. N. Hill (ed.), The Sea. Wiley, New York, p. 507-528.

Boas, M. L., 1983. Mathematical Methods in the Physical Sciences. (2nd edn.) New York: Wiley, $793 \mathrm{pp}$.

Boon, J. D., 1975. Tidal discharge asymmetry in a salt marsh drainage system. Limnology and Oceanography, 20: 71-80.

Boon, J. D., and R. J. Byrne, 1981. On basin hypsometry and the morphodynamic response of coastal inlet systems. Marine Geology, 40: 27-48.

Bruun, P., 1954. Coast erosion and the development of beach profiles. U.S. Army Corps of Engineers, Beach Erosion Board, Tech. Memo. 44, 79 pp.

Bruun, P., 1967. Tidal inlets housekeeping. ASCE Journal of the Hydraulics Division, 93 (HY5): 167-184.

Bruun, P., 1976. Port Engineering. (2nd edn.) Houston, TX: Gulf Publishing, 586 pp.

Bruun, P., and F. Gerritsen, 1960. Stability of Tidal Inlets. Amsterdam: North Holland Publishing Company, 130 pp.

Burke, R. W., and K. D. Stolzenbach, 1983. Free surface flow through salt marsh grass. Massachusetts Institute of Technology Sea Grant College Program, MITSG 8316, Cambridge, MA, $252 \mathrm{pp}$. 
Byrne, R. J., P. Bullock and D. G. Tyler, 1975. Response characteristics of a tidal inlet: a case study. In: L. E. Cronin (ed.), Estuarine Research, Volume II: Geology and Engineering. Academic Press, New York, p. 201-216.

Byrne, R. J., R. A. Gammisch and G. R. Thomas, 1981. Tidal prism-inlet area relations for small tidal inlets. In: Proceedings of the 17th International Conference on Coastal Engineering. American Society of Civil Engineers, New York, p. 25172533.

Chantler, A. G., 1974. The applicability of regime theory to tidal watercourses. Journal of Hydraulic Research, 12: 181-191.

Chappell, J., and I. G. Eliot, 1979. Surf-beach dynamics in time and space -- an Australian case study, and elements of a predictive model. Marine Geology, 32: 231-250.

Davies, J. L., 1980. Geographical Variation in Coastal Development. (2nd edn.) London: Longman, $212 \mathrm{pp}$.

de Jong, H., and F. Gerritsen, 1985. Stability parameters of the Western Scheldt estuary. In: Proceedings of the 19th International Conference on Coastal Engineering. American Society of Civil Engineers, New York, p. 3079-3093.

de Jonge, V. N., 1992. Tidal flow and residual flow in the Ems estuary. Estuarine Coastal and Shelf Science, 34: 1-22.

Dean, R. G., 1977. Equilibrium beach profiles. University of Delaware, Ocean Engineering Tech. Rep. 12, Newark, DE, 46 pp.

Dieckmann, R., M. Osterthun and H. W. Partenscky, 1987. Influence of water-level elevation and tidal range on the sedimentation in a German tidal flat area. Progress in Oceanography, 18: 151-166.

DiLorenzo, J. L., 1988. The overtide and filtering response of small inlet/bay systems. In: D. G. Aubrey and L. Weishar (ed.), Hydrodynamics and Sediment Dynamics of Tidal Inlets. Coastal and Estuarine Studies, Vol. 29, Springer-Verlag, New York, p. 24-53.

Diplas, P., 1990. Characteristics of self-formed straight channels. Journal of Hydraulic Engineering, A.S.C.E., 116 (HY5): 707-728.

Dyer, K. R., 1986. Coastal and estuarine sediment dynamics. New York: Wiley, 342 pp. Dronkers, J., 1986. Tide-induced residual transport of fine sediment. In: J. van de Kreeke (ed.), Physics of shallow estuaries and bays. Coastal and Estuarine Studies, Vol. 16, Springer-Verlag, New York, p. 228-244. 
Einstein, H. A., 1950. The bed-load function for sediment transportation in open channel flows. U.S. Department of Agriculture, Technical Bulletin No. 1026, Washington, D.C.

Eiser, W. C., and B. Kjerfve, 1986. Marsh topography and hypsometric characteristics of a South Carolina salt marsh basin. Estuarine, Coastal and Shelf Science, 23: 595605.

Engelund, F., and J. Fredsoe, 1982. Sediment ripples and dunes. Annual Review of Fluid Mechanics, 14: 13-37.

Escoffier, F. F., 1977. Hydraulics and stability of tidal inlets. U.S. Army Coastal Engineering Research Center, G.I.T.I. Report 13, Vicksburg, MI, 72 pp.

Evans, G., and M. B. Collins, 1975. The transportation and deposition of suspended sediment over the interidal flats of the Wash. In: J. Hails and A. Carr (ed.), Nearshore Sediment Dynamics and Sedimentation. Wiley, London, p. 273-306.

FitzGerald, D. M., and D. Nummedal, 1983. Response characteristics of an ebbdominated tidal inlet channel. Journal of Sedimentary Petrology, 53: 833-845.

Fredsoe, J., 1982. Shape and dimensions of stationary dunes in rivers. Journal of the Hydraulics Division, ASCE, 108 (HY8): 932-947.

Frey, R. W., and P. B. Basan, 1985. Coastal salt marshes. In: R. A. Davis (ed.), Coastal Sedimentary Environments. Springer-Verlag, New York, p. 225-302.

Friedrichs, C. T., and D. G. Aubrey, 1988. Non-linear tidal distortion in shallow wellmixed estuaries: a synthesis. Estuarine, Coastal and Shelf Science, 27: 521-545.

Friedrichs, C. T., D. G. Aubrey, G. S. Giese and P. E. Speer, 1993. Hydrodynamical modeling of a multiple-inlet barrier beach/estuary system: insight into tidal inlet formation and stability. In: D. G. Aubrey and G. S. Giese (ed.), Formation and Evolution of Multiple Inlet Systems. Coastal and Estuarine Sciences Series, American Geophysical Union, Washington, D.C., in press.

Friedrichs, C. T., D. G. Aubrey and D. R. Lynch, 1990. Non-linear tides in twodimensions in frictionally-dominated embayments. Eos, 71: 1370.

Friedrichs, C. T., D. G. Aubrey and W. S. Spencer, in prep., a, Great Bay, New Hampshire, tidal flat field study: preliminary data report. Woods Hole Oceanographic Institution, Technical Report, Woods Hole, MA.

Friedrichs, C. T., D. R. Lynch and D. G. Aubrey, 1992. Velocity asymmetries in frictionally-dominated tidal embayments: longitudinal and lateral variability. In: D. Prandle (ed.), Dynamics and Exchanges in Estuaries and the Coastal Zone. Coastal and Estuarine Sciences Series, American Geophysical Union, Washington, D.C., p. 277-312. 
Friedrichs, C. T., D. R. Lynch and D. G. Aubrey, in prep., b, Two-dimensional patterns of bottom shear stress in shallow tidal embayments with intertidal flats.

Friedrichs, C. T., and O. S. Madsen, 1992. Nonlinear diffusion of the tidal signal in frictionally dominated embayments. Journal of Geophysical Research, 97: 56375650 .

Fry, V. A., and D. G. Aubrey, 1990. Tidal velocity asymmetries and bedload transport in shallow embayments. Estuarine, Coastal and Shelf Science, 30: 453-473.

Gallagher, B. S., and W. H. Munk, 1971. Tides in shallow water: spectroscopy. Tellus, 23: 346-363.

Gardner, L. R., and M. Bohn, 1980. Geomorphic and hydraulic evolution of tidal creeks on a subsiding beach ridge plain, North Inlet, S.C. Marine Geology, 34: M91M97.

George, K. J., 1975. The tides and tidal streams of the Tamar estuary. PhD Thesis, University of London, London, England, $550 \mathrm{pp.}$

Gerritsen, F., H. de Jong and A. Langerak, 1991. Cross-sectional stability of estuary channels in the Netherlands. In: B. L. Edge (ed.), Proceedings of the 22nd International Conference on Coastal Engineering. American Society of Civil Engineers, New York, p. 2922-2935.

Giese, B. S., and D. A. Jay, 1989. Modelling tidal energetics of the Columbia River estuary. Estuarine, Coastal and Shelf Science, 29: 549-571.

Godin, G., 1991. Frictional effects in river tides. In: B. B. Parker (ed.), Tidal Hydrodynamics. Wiley, New York, p. 379-402.

Goodwin, C. R., E. W. Emmett and B. Glenne, 1970. Tidal study of three Oregon estuaries. Oregon State University Engineering Experiment Station, Bulletin No. 45, Corvallis, OR, $45 \mathrm{pp}$.

Grant, W. D., and O. S. Madsen, 1979. Combined wave and current interaction with a rough bottom. Journal of Geophysical Research, 84: 1797-1808.

Harleman, D. R. F., 1966. Tidal dynamics in estuaries, part II: real estuaries. In: A. T. Ippen (ed.), Estuary and Coastline Hydrodynamics. McGraw-Hill, New York, p. 522-545.

Hiug, F. W., 1976. Post-glacial stratigraphy of the Great Bay estuary system. Masters Thesis, University of New Hampshire, 90 pp.

Hayami, S., 1951. On the propagation of flood waves. Bulletin of the Disaster Prevention Research Institute, Kyoto, Japan, Vol. 1, 1-16 pp.

Hayes, M. O., 1979. Barrier island morphology as a function of tidal and wave regime. In: S. P. Leatherman (ed.), Barrier Islands. Academic Press, New York, p. 1-27. 
Henderson, F. M., 1966. Open Channel Flow. New York: Macmillan, 522 pp.

Hunt, J. N., 1964. Tidal oscillations in estuaries. Geophysical Journal of the Royal Astronomical Society, 8: 440-455.

Ikeda, S., 1982. Incipient motion of sand particles on side slopes. Journal of the Hydraulics Division, ASCE, 108 (HY1): 95-114.

Ippen, A. T., 1966. Tidal dynamics in estuaries, part I: estuaries of rectangular section. In: A. T. Ippen (ed.), Estuary and Coastline Hydrodynamics. McGraw-Hill, New York, p. 493-522.

Jay, D. A., 1991. Green's Law revisited: tidal long-wave propagation in channels with strong topography. Journal of Geophysical Research, 96: 20,585-20,598.

Kabbaj, A., and C. Le Provost, 1980. Nonlinear tidal waves in channels: a perturbation method adapted to the importance of quadratic bottom friction. Tellus, 32: 143163.

Kapdasli, M. S., and K. R. Dyer, 1986. Threshold conditions for sand movement on a rippled bed. Geo-Marine Letters, 6: 161-164.

Kirby, R., 1992. Effects of sea-level on muddy coastal margins. In: D. Prandle (ed.), Dynamics and Exchanges in Estuaries and the Coastal Zone. Coastal and Estuarine Sciences Series, American Geophysical Union, Washington, D.C., p. 313-334.

Klein, G. D., 1985. Intertidal flats and intertidal sand bodies. In: R. A. Davis (ed.), Coastal Sedimentary Environments. Springer-Verlag, New York, p. 187-218.

Kojima, H., and S. D. Hunt, 1980. Fort George Inlet, Glossary of Inlets Report No. 10. University of Florida, F.S.G.C. Report 38, Gainesville, FL, 52 pp.

Kreiss, H., 1957. Some remarks about nonlinear oscillations in tidal channels. Tellus, 9: 53-68.

Krishnamurthy, M., 1977. Tidal prism of equilibrium inlets. Journal of the Waterway, Port, Coastal and Ocean Division, ASCE, 103 (WW4): 423-432.

Lane, E. W., 1955. Design of stable channels. Transactions, American Society of Civil Engineers, 120: 1234-1260.

LeBlond, P. H., 1978. On tidal propagation in shallow rivers. Journal of Geophysical Research, 83: 4717-4721.

Lewis, R. E., and J. O. Lewis, 1987. Shear stress variations in an estuary. Estuarine, Coastal and Shelf Science, 25: 621-635.

Li, W.-H., 1974. Well-mixed estuaries with nonlinear resistance. Journal of Hydraulic Research, 12: 83-98.

McDowell, D. M., and B. A. O'Connor, 1977. Hydraulic Behaviour of Estuaries. New York: Wiley, 292 pp. 
Mehta, A. J., 1984. Characterization of cohesive sediment properties and transport processes in estuaries. In: A. J. Mehta (ed.), Estuarine Cohesive Sediment Dynamics. Coastal and Estuarine Studies Series, Vol. 14, Springer-Verlag, New York, p. 290-325.

Münchow, A., and R. W. Garvine, 1991. Non-linear, barotropic tides and bores in shallow estuaries. Tellus, 43A: 246-256.

Myrick, R. M., and L. B. Leopold, 1963. Hydraulic geometry of a small tidal estuary. USGS Professional Paper, 442-B: 18 pp.

Nichols, M. M., and R. B. Biggs, 1985. Estuaries. In: R. A. Davis (ed.), Coastal sedimentary environments. Springer-Verlag, New York, p. 77-186.

NOS, 1985. Index of tide stations, United States of America and miscellaneous other - locations. NOAA/National Ocean Service, Rockville, MD, 143 pp.

Nummedal, D., and S. M. Humphries, 1978. Hydraulics and dynamics of North Inlet, 1975-76. US Army Coastal Engineering Research Center, GITI Report 16, Ft. Belvoir, VA, 214 pp.

O'Brien, M. P., 1931. Estuary tidal prism related to entrance areas. Civil Engineering, 1: 738-739.

O'Connor, B., J. Nicholson and R. Rayner, 1991. Estuary geometry as a function of tidal range. In: B. L. Edge (ed.), Proceedings of the 22nd International Conference on Coastal Engineering. American Society of Civil Engineers, New York, p. 30503062.

Officer, C. B., 1976. Physical Oceanography of Estuaries and Associated Coastal Waters. New York: Wiley, 463 pp.

Oldale, R. N., 1979. Glacial deposits and features of outer Cape Cod. In: S. P. Leatherman (ed.), Environmental Geologic Guide to Cape Cod National Seashore. National Park Service Cooperative Resarch Unit, University of Massachusetts, Amherst, MA, p. 41-54.

Paola, C., 1983. Flow and skin friction over natural rough beds. Ph.D. Thesis, Woods Hole Oceanographic Institution - Massachusetts Institute of Technology Joint Program in Oceanography, Woods Hole, MA, 347 pp.

Park, M., 1985. Prediction of tidal hydraulics and sediment transport patterns in Stony Brook Harbor. M.S. Thesis, State University of New York, Stony Brook Marine Environmental Science Program, Stony Brook, NY, 146 pp.

Parker, B. B., 1984. Frictional effects on the tidal dynamics of a shallow estuary. Ph.D. Thesis, Johns Hopkins University, Baltimore, MD, 292 pp. 
Parker, B. B., 1991. The relative importance of the various nonlinear mechanisms in a wide range of tidal interactions (review). In: B. B. Parker (ed.), Tidal Hydrodynamics. Wiley, New York, p. 237-268.

Parker, G., 1978. Self-formed straight rivers with equilibrium banks and mobile bed, part 1: the sand-silt river. Journal of Fluid Mechanics, 89: 109-125.

Partheniades, E., 1965. Erosion and deposition of cohesive soils. Journal of the Hydraulics Division, ASCE, 91 (HY1): 105-139.

Perroud, P., 1959. The propagation of tidal waves into channels of gradually varying cross-section. U.S. Army Corps of Engineers Beach Erosion Board, Technical Memo. 112, Washington, D.C., 27 pp.

Perry, F. C., W. C. Seabergh and E. F. Lane, 1978. Improvements for Murrells Inlet, South Carolina. US Army Engineer Waterways Experiment Station, T.R. H-78-4, Vicksburg, MI, 339 pp.

Pestrong, R., 1965. The development of drainage patterns on tidal marshes. Stanford University publications in the geological sciences, Vol. 10, No. 2, Stanford, CA, $87 \mathrm{pp}$.

Pethick, J. S., 1980. Velocity surges and asymmetry in tidal channels. Estuarine and Coastal Marine Science, 11:331-345.

Pillsbury, G. B., 1939. Tidal Hydraulics. Washington, D.C.: U.S. Government Printing Office, $283 \mathrm{pp}$.

Pingree, R. D., and D. K. Griffiths, 1979. Sand transport paths around the British Isles resulting from $\mathrm{M}_{2}$ and $\mathrm{M}_{4}$ tidal interactions. Journal of the Marine Biological Association of the U.K., 59: 497-513.

Pingree, R. D., and L. Maddock, 1978. The $\mathrm{M}_{4}$ tide in the English Channel derived from a non-linear numerical model of the $\mathrm{M}_{2}$ tide. Deep-Sea Research, 25: 53-68.

Ponce, V. M., R. M. Li and D. B. Simons, 1978. Applicability of kinematic and diffusion models. Journal of the Hydraulics Division, ASCE, 104 (HY3): 353-360.

Postma, H., 1961. Hydrography of the Dutch Wadden Sea. Netherlands Journal of Sea Research, 1: 148-190.

Postma, H., 1967. Sediment transport and sedimentation in the estuarine environment. In: G. H. Lauff (ed.), Estuaries. American Assocation for the Advancement of Science, Washington, D.C., p. 158-179.

Prandle, D., 1980. Modelling of tidal barrier schemes: an analysis of the open-boundary problem by reference to AC circuit theory. Estuarine and Coastal Marine Science, 11: 53-71. 
Prandle, D., and M. Rahman, 1980. Tidal response in estuaries. Journal of Physical Oceanography, 10: 1552-1573.

Redfield, A. C., 1965. Ontogeny of a salt marsh. In: G. H. Lauff (ed.), Estuaries. American Assocation for the Advancement of Science, Washington, D.C., p. 108114.

Riedel, H. P., and M. R. Gourlay, 1981. Inlets/estuaries discharging into sheltered waters. In: Proceedings of the 17th International Conference on Coastal Engineering. American Society of Civil Engineers, New York, p. 2550-2564.

Robinson, I. S., L. Warren and J. F. Longbottom, 1983. Sea-level fluctuations in the Fleet, an English tidal lagoon. Estuarine, Coastal and Shelf Science, 16: 651-668.

Roman, C. T., K. W. Able, M. A. Lazzari and K. L. Heck, 1990. Primary productivity of angiosperm and macroalgae dominated habitats in a New England Salt Marsh: a comparative analysis. Estuarine Coastal and Shelf Science, 30: 35-45.

Shetye, S. R., and A. D. Gouveia, 1992. On the role of geometry of cross-section in generating flood-dominance in shallow estuaries. Estuarine, Coastal and Shelf Science, 35: 113-126.

Smith, J. D., 1977. Modeling of sediment transport on continental shelves. In: E. D. Goldberg (ed.), The Sea, Vol. 6. Wiley, New York, p. 539-577.

Smith, J. D., and S. R. McLean, 1977. Spatially averaged flow over a wavy surface. Joumal of Geophysical Research, 82: 1735-1746.

Speer, P. E., 1984. Tidal distortion in shallow estuaries. Ph.D. Thesis, Woods Hole Oceanographic Institution - Massachusetts Institute of Technology Joint Program in Oceanography, Woods Hole, MA, 210 pp.

Speer, P. E., and D. G. Aubrey, 1985. A study of non-linear tidal propagation in shallow inlet/estuarine systems, part II: theory. Estuarine, Coastal and Shelf Science, 21: 207-224.

Speer, P. E., D. G. Aubrey and C. T. Friedrichs, 1991. Nonlinear hydrodynamics of shallow tidal inlet/bay systems. In: B. B. Parker (ed.), Tidal Hydrodynamics. Wiley, New York, p. 321-340.

Stephens, J. A., R. J. Uncles, M. L. Barton and F. Fitzpatrick, 1992. Bulk properties of intertidal sediments in a muddy, macrotidal estuary. Marine Geology, 103: 445460.

Strahler, A. N., 1952. Hypsometric (area-altitude) analysis of erosional topography. Geological Society of America Bulletin, 63: 117-1142.

Swift, M. R., and W. S. Brown, 1983. Distribution of bottom stress and tidal energy in a well-mixed estuary. Estuarine, Coastal and Shelf Science, 17: 297-317. 
Uncles, R. J., 1981. A note on tidal asymmetry in the Severn estuary. Estuarine, Coastal and Shelf Science, 13: 419-432.

Uncles, R. J., R. C. A. Elliott and S. A. Weston, 1985. Lateral distributions of water, salt and sediment transport in a partly mixed estuary. In: B. L. Edge (ed.), Proceedings of the 19th International Conference on Coastal Engineering. American Society of Civil Engineers, New York, p. 3067-3077.

Uncles, R. J., and J. A. Stephens, 1989. Distributions of suspended sediment at high water in a macrotidal estuary. Journal of Geophysical Research, 94: 14,39514,405 .

Uncles, R. J., and J. A. Stephens, 1990. Computed and observed currents, elevations, and salinity in a branching estuary. Estuaries, 13: 133-144.

van de Kreeke, J., 1967. Water level fluctuations and flow in tidal inlets. Journal of Waterways and Harbors Division, ASCE, 43 (WW2): 97-106.

van de Kreeke, J., 1990. Can multiple tidal inlets be stable? Estuarine, Coastal and Shelf Science, 30: 261-273.

Wallis, S. G., and D. W. Knight, 1984. Calibration studies concerning a one-dimensional numerical tidal model with particular reference to resistance coefficients. Estuarine Coastal and Shelf Science, 19: 541-562.

Weisman, R. N., G. P. Lennon and F. E. Schuepfer, 1990. Resistence coefficient in a tidal channel. In: M. L. Spaulding (ed.), Estuarine and Coastal Modeling. American Society of Civil Engineers, New York, p. 123-131.

Wells, J. T., C. E. Adams, Y.-A. Park and E. W. Frankenberg, 1990. Morphology, sedimentology and tidal channel processes on a high-tide-range mudflat, west coast of Korea. Marine Geology, 95: 111-130.

Wells, J. T., and Y.-A. Park, 1992. Observations on shelf and subtidal channel flow: implications of sediment dispersal seaward of the Keum River estuary, Korea. Estuarine, Coastal and Shelf Science, 34: 365-379.

Westerink, J. J., K. D. Stolzenbach and J. J. Conner, 1989. General spectral computations of the nonlinear shallow water tidal interactions within the Bight of Abaco. Journal of Physical Oceanography, 19: 1348-1371.

Wong, K.-C., 1989. Tidally generated residual currents in a sea level canal or tidal strait with constant breadth and depth. Journal of Geophysical Research, 94: 81798192.

Wright, L. D., J. M. Coleman and B. G. Thom, 1973. Processes of channel development in a high-tide range environment: Cambridge Gulf-Ord River delta, Western Australia. Journal of Geology, 81: 15-41. 
Wright, L. D.; J. M. Coleman and B. G. Thom, 1975. Sediment transport and deposition in a macrotidal river channel: Ord River, Western Australia. In: L. E. Cronin (ed.), Estuarine Research, Volume II: Geology and Engineering. Academic Press, New York, p. 309-321.

Wright, L. D., P. Nielsen, A. D. Short and M. O. Green, 1982. Morphodynamics of a macrotidal beach. Marine Geology, 50: 97-128.

Wright, L. D., D. B. Prior, C. H. Hobbs, R. J. Byrne, J. D. Boon, L. C. Schaffner and M. O. Green, 1987. Spatial variability of bottom types in the lower Chesapeake Bay and adjoining estuaries and inner shelf. Estuarine, Coastal and Shelf Science, 24: 765-784.

Wright, L. D., A. D. Short and M. O. Green, 1985. Short-term changes in the morphodynamic states of beaches and surf zones: an empirical predictive model. Marine Geology, 62: 339-364.

Yalin, M. S., 1977. Mechanics of Sediment Transport. (2nd edn.) New York: Pergamon, $298 \mathrm{pp}$.

Young, H. D., 1962. Statistical treatment of experimental data. New York: McGraw-Hill, $172 \mathrm{pp}$.

Zimmerman, J. T. F., 1973. The influence of the subaqueous profile on wave induced bottom stress. Netherlands Journal of Sea Research, 6: 542-549.

Zimmerman, J. T. F., 1978. Topographic generation of residual circulation by oscillatory (idal) currents. Geophysical and Astrophysical Fluid Dynamics, 11: 35-47. 


\section{DOCUMENT LIBRARY}

February 5, 1993

\section{Distribution List for Technical Report Exchange}

University of California, San Diego

SIO Library 0175C (TRC)

9500 Gilman Drive

La Jolla, CA 92093-0175

Hancock Library of Biology \&

Oceanography

Alan Hancock Laboratory

University of Southern California

Univèrsity Park

Los Angeles, CA 90089-0371

Gifts \& Exchanges

Library

Bedford Institute of Oceanography

P.O. Box 1006

Dartmouth, NS, B2Y 4A2, CANADA

Office of the International

Ice Patrol

c/o Coast Guard R \& D Center

Avery Point

Groton, CT 06340

NOAA/EDIS Miami Library Center

4301 Rickenbacker Causeway

Miami, FL 33149

Library

Skidaway Institute of Oceanography

P.O. Box 13687

Savannah, GA 31416

Institute of Geophysics

University of Hawaii

Library Room 252

2525 Correa Road

Honolulu, HI 96822

Marine Resources Information Center

Building E38-320

MIT

Cambridge, MA 02139

Library

Lamont-Doherty Geological

Observatory

Columbia University

Palisades, NY 10964

Library

Serials Department

Oregon State University

Corvallis, OR 97331
Pell Marine Science Library

University of Rhode Island

Narragansett Bay Campus

Narragansett, RI 02882

Working Collection

Texas A\&M University

Dept. of Oceanography

College Station, TX 77843

Fisheries-Oceanography Library 151 Oceanography Teaching Bldg.

University of Washington

Seattle, WA 98195

Library

R.S.M.A.S.

University of Miami

4600 Rickenbacker Causeway

Miami, FL 33149

Maury Oceanographic Library

Naval Oceanographic Office

Stennis Space Center

NSTL, MS 39522-5001

Marine Sciences Collection

Mayaguez Campus Library

University of Puerto Rico

Mayaguez, Puerto Rico 00708

Library

Institute of Oceanographic Sciences

Deacon Laboratory

Wormley, Godalming

Surrey GU8 5UB

UNITED KINGDOM

The Librarian

CSIRO Marine Laboratories

G.P.O. Box 1538

Hobart, Tasmania

AUSTRALIA 7001

Library

Proudman Oceanographic Laboratory

Bidston Observatory

Birkenhead

Merseyside L43 7 RA

UNITED KINGDOM

IFREMER

Centre de Brest

Service Documentation - Publications

BP 7029280 PLOUZANE

FRANCE 


\begin{tabular}{|c|c|c|c|}
\hline $\begin{array}{c}\text { REPORT DOCUMENTATION } \\
\text { PAGE } \\
\end{array}$ & $\begin{array}{l}\text { 1. REPORT NO. } \\
\text { WHOI-93-17 }\end{array}$ & 2. & 3. Recipient's Accession No. \\
\hline \multirow{2}{*}{\multicolumn{3}{|c|}{$\begin{array}{l}\text { 4. Title and Subtitle } \\
\text { Hydrodynamics and Morphodynamics of Shallow Tidal Channels and Intertidal Flats }\end{array}$}} & $\begin{array}{l}\text { 5. Report Date } \\
\text { February } 1993\end{array}$ \\
\hline & & & 6. \\
\hline \multicolumn{3}{|l|}{$\begin{array}{l}\text { 7. Author(s) } \\
\text { Carl T. Friedrichs }\end{array}$} & 8. Performing Organization Rept. No. \\
\hline \multirow{2}{*}{\multicolumn{3}{|c|}{$\begin{array}{l}\text { Woods Hole Oceanographic Institution } \\
\text { Woods Hole, Massachusetts } 02543\end{array}$}} & $\begin{array}{l}\text { 10. Project/TaskWork Unit No. } \\
\text { WHOI-93-17 }\end{array}$ \\
\hline & & & $\begin{array}{l}\text { 11. Contract(C) or Grant(G) No. } \\
\text { (C) OCE91-02429 } \\
\text { (G) }\end{array}$ \\
\hline \multirow{2}{*}{\multicolumn{3}{|c|}{$\begin{array}{l}\text { 12. Sponsoring Organization Name and Address } \\
\text { Funding was provided by the Office of Naval Research through the American Society for } \\
\text { Engineering Education and the National Science Foundation under grant OCE91-02429. }\end{array}$}} & $\begin{array}{l}\text { 13. Type of Report \& Period Covered } \\
\text { Ph.D. Thesis }\end{array}$ \\
\hline & & & 14. \\
\hline
\end{tabular}

\section{Supplementary Notes}

This thesis should be cited as: Carl T. Friedrichs, 1993. Hydrodynamics and Morphodynamics of Shallow Tidal Channels and Intertidal Flats. Ph.D. Thesis. MIT/WHOI, WHOI-93-17.

\section{Abstract (Limit: 200 words)}

In this thesis, mechanisms which control morphodynamics of shallow tidal embayments are investigated analytically. In the process of exploring these mechanisms (specifically asymmetries in bottom stress, $\tau$ ), basis momentum and mass balances which govern flow in these systems are clarified. Temporal asymmetries in $\tau$ are investigated via a new perturbation scheme which quantifies nonlinear processes and combines geometric controls on asymmetry into a single non-dimensional parameter. Implications of spatial asymmetries in $\tau$ are investigated through stability criteria based on a uniform distribution of $\tau$. Morphologic observations of both tidal channels and intertidal flats are consistent with a uniform distribution of $\tau$ at equilibrium. Investigation of morphodynamic mechanisms leads to scalings of momentum and continuity which diverge from classical models. Scalings for prismatic channels with strong tidal asymmetries indicate friction often dominates acceleration in the momentum equation. The resulting "zero-inertia" balance gives a time-varying diffusion equation which requires along-channel amplitude to decay. Uniform $\tau$ justifies a new scaling of continuity for exponentially-shaped channels. In such channels, along-channel gradients in tidal velocity are small and are often dominated by gradients in cross-sectional area. The resulting first-order wave equation allows only constant amplitude, forward propagating waveforms which are independent of channel length.

\section{Document Analysis a. Descriptors}

tidal channels

hydrodynamics

tidal flats

b. Identifiers/Open-Ended Terms

c. COSATI Field/Group

18. Availability Statement

Approved for publication; distribution unlimited.

\begin{tabular}{|l|l|}
\hline $\begin{array}{c}\text { 19. Security Class (This Report) } \\
\text { UNCLASSIFIED }\end{array}$ & $\begin{array}{c}\text { 21. No. of Pages } \\
218\end{array}$ \\
\hline 20. Security Class (This Page) & 22. Price \\
\hline
\end{tabular}

